\title{
Chiral Primary Amine/Ketone Cooperative Catalysis for Asymmetric $\alpha$-Hydroxylation with Hydrogen Peroxide
}

\author{
Mao Cai ${ }^{a b}$ Kaini Xu, ${ }^{c}$ Yuze Li,${ }^{b d}$ Zongxiu Nie ${ }^{b d}$ Long Zhang*c and Sanzhong Luo ${ }^{* c}$ \\ ${ }^{a}$ Key Laboratory of Molecular Recognition and Function, Institute of Chemistry, Chinese \\ Academy of Sciences, Beijing 100190, China \\ ${ }^{b}$ School of Chemical Science, University of Chinese Academy of Sciences, Beijing, 100049, China \\ ${ }^{c}$ Center of Basic Molecular Science, Department of Chemistry, Tsinghua University, Beijing, \\ 100084, China \\ ${ }^{d}$ Beijing National Laboratory for Molecular Sciences, Key Laboratory for Analytical Chemistry \\ for Living Biosystems, Institute of Chemistry, the Chinese Academy of Sciences, Beijing 100190, \\ China
}

\section{Supplementary Information}

\section{Supplementary Methods}

1. General Considerations.

2. Substrate and Reagent Synthesis

3. Optimizaiton Details

4. $\alpha$-Hydroxylation Reactions and Compound Characterization

5. Determination of Absolute Configuration

6. Synthesis of Biologically Active Compounds

7. Isotopic Lablling

8. Kinetics Study

9. Computational Investigations 


\section{Supplementary Methods}

1. Copies of NMR Spectra

2. Copies of HPLC Data S95

Supplementary References S137

\section{General Considerations}

Unless noted, materials were purchased from commercial source and used without further purification. ${ }^{1} \mathrm{H}$ and ${ }^{13} \mathrm{C}$ NMR spectra were recorded on a Bruker Ultra Shield $400 \mathrm{MHz}$ spectrometers $\left({ }^{1} \mathrm{H}\right.$ NMR frequency, corresponding ${ }^{13} \mathrm{C}$ frequencies are $\left.100 \mathrm{MHz}\right)$ and spectra were referenced to the solvent ( ${ }^{1} \mathrm{H}$ NMR: $\mathrm{CDCl}_{3}$ at $7.26 \mathrm{ppm} ;{ }^{13} \mathrm{C} \mathrm{NMR}: \mathrm{CDCl}_{3}$ at $77.16 \mathrm{ppm}$ ). Data for ${ }^{1} \mathrm{H}$ NMR spectra are reported as follows: chemical shift, multiplicity $(\mathrm{s}=\operatorname{singlet}, \mathrm{d}=$ doublet, $\mathrm{t}=$ triplet, $\mathrm{m}=$ multiplet, $\mathrm{td}=$ triplet of doublet, $\mathrm{dt}=$ doublet of triplet, $\mathrm{dd}=$ doublet of doublet), coupling constants $(\mathrm{Hz})$, and integration. Coupling constants (J values) were calculated directly from the spectra. Infrared Spectroscopy was collected on a Thermo Fisher Nicolet 6700. High resolution mass spectra were obtained using electrospray ionization (ESI) mass spectrometer. Mass spectrometry experiments were performed on an Impact HD Q-TOF mass spectrometer (Bruker Daltonics, Bremen, Germany), operated in the positive ion mode. Nano-ESI tips were pulled from the borosilicate glass capillaries $(\mathrm{O}$-tip) $(\mathrm{i} . \mathrm{d} .=0.86 \mathrm{~mm}$, o.d. $=1.5 \mathrm{~mm}$ ). A direct-current (DC) high voltage of $2500 \mathrm{~V}$ was supplied to the rear of the capillary and was powered by a high voltage generator. The enantiomeric excesses were determined by HPLC analysis on Chiral Daicel Chiralpak OD-H, AD-H and AS-H or chiral stationary phases (CP-Chirasil-Dex CB: $25 \mathrm{~m} \cdot 0.25 \mathrm{~mm})$. Optical rotations were measured on a commercial 
polarimeter and reported as follows: $[\alpha]_{\mathrm{D}}^{\mathrm{T}}\left(\mathrm{c}=\mathrm{g} / 100 \mathrm{~mL}, \mathrm{CHCl}_{3}\right)$. Analytical thin layer chromatography (TLC) was visualized with UV light, and stained with $\mathrm{KMNO}_{4}$ or phenylhydrazine solutions.

\section{Synthesis of Substrate and Reagent}

$\beta$-Ketoesters 1a-1f, 1i-1k, 1m and 1t were prepared by alkylation of the corresponding $\alpha$-unsubstituted $\beta$-ketoesters with alkyl iodid ${ }^{1} . \beta$-Ketoesters $1 \mathbf{r}$ was prepared by allylation of the corresponding acetoacetate with allyl bromide ${ }^{1} . \beta$-Ketoesters $1 \mathbf{l}^{2}, \mathbf{1 n}-\mathbf{1} \mathbf{q}^{2}$ and $\mathbf{1} \mathbf{s}^{3}$ were prepared according to literature precedent. The cyclic $\beta$-ketoesters $1 \mathbf{u}^{4}, \mathbf{1 v}^{4}$ and cyclic $\beta$-ketoamides ${ }^{5}$ were prepared according to the corresponding literature. Acyclic $\beta$-ketoamides were prepared according to a known procedure ${ }^{6} . \beta$-Ketoamide $\mathbf{5 0 - 5 q}$ was prepared according to literature precedent ${ }^{7}$. $\beta$-Ketoesters $\mathbf{1 g}-\mathbf{1 h}$ and $\mathbf{5 r}-\mathbf{5 v}$ were prepared by transesterification of the corresponding alcohol with $\mathbf{1} \mathbf{b}^{8}$.

Typical Procedure for the Synthesis of Catalyst 3a: The aminocatalyst 3a was synthesized similar to the procedure reported previously by our group9. To a solution of N-Boc-L-tert-leucine (23.15 g, $100 \mathrm{mmol})$ in anhydrous $\mathrm{CH}_{2} \mathrm{Cl}_{2}(200 \mathrm{~mL})$ at $0{ }^{\circ} \mathrm{C}$, was added N,N-Diisopropylethylamine (DIPEA, $28.43 \mathrm{~g}, 220 \mathrm{mmol}$ ) followed by HBTU (41.72 g, 110 mmol). After stirring for $30 \mathrm{~min}$ at room temperature, ethylamine $(4.51 \mathrm{~g}, 100 \mathrm{mmol})$ in anhydrous $\mathrm{CH}_{2} \mathrm{Cl}_{2}(20 \mathrm{~mL})$ was added under ice bath, and the solution was stirred at room temperature for an additional $12 \mathrm{~h}$. The reaction mixture was washed with $\mathrm{HCl}(0.1 \mathrm{M})$, water, saturated $\mathrm{NaHCO}_{3}$ and brine, then dried with anhydrous $\mathrm{Na}_{2} \mathrm{SO}_{4}$. After removal of the solvent, the residue was purified by chromatography with petroleum ether and ethyl acetate. To the obtained 
product was added $\mathrm{CH}_{2} \mathrm{Cl}_{2}$, then $35 \mathrm{~mL}$ TFA at $0{ }^{\circ} \mathrm{C}$ and stirred overnight. The solvent was removed to $100 \mathrm{~mL}$, and $\mathrm{H}_{2} \mathrm{O}(50 \mathrm{~mL})$ were added. Keep the aqueous phase after separation and add another $50 \mathrm{~mL} \mathrm{CH}_{2} \mathrm{Cl}_{2}$. The $\mathrm{pH}$ value of the solvent was adjusted to 12 by the addition of $\mathrm{K}_{2} \mathrm{CO}_{3}$ under ice bath. The aqueous phase was extracted with $\mathrm{CH}_{2} \mathrm{Cl}_{2}(40 \mathrm{~mL})$, and the organic layers were combined and dried with anhydrous $\mathrm{Na}_{2} \mathrm{SO}_{4}$. After removal of the solvent, the intermediate compound $(9.15 \mathrm{~g}, 58 \%$ yield $)$ was directly used for the next step without purification. To the above-obtained intermediate $(9.15 \mathrm{~g})$ in anhydrous THF $(150 \mathrm{~mL})$ at $0{ }^{\circ} \mathrm{C}$ was added $\mathrm{LiAlH}_{4}(5.51 \mathrm{~g}, 145 \mathrm{mmol})$ in portions, then the solution was stirred at room temperature for 10 minutes and heated to reflux for $6 \mathrm{~h}$. After cooling to room temperature, $10 \mathrm{~mL}$ water was added at $0{ }^{\circ} \mathrm{C}$, followed by $15 \%$ aqueous $\mathrm{NaOH}(10 \mathrm{~mL})$, and anhydrous $\mathrm{Na}_{2} \mathrm{SO}_{4}$ after 10 minutes. The solid formed was filtered and washed with ethyl acetate several times. The solvent was removed, and pure catalyst $3 \mathbf{a}(5.85 \mathrm{~g})$ was obtained as a colorless oil by distillation under reduced pressure (70 \% yield). ${ }^{1} \mathbf{H}$ NMR $\left(400 \mathrm{MHz}, \mathrm{CDCl}_{3}\right) \delta 2.78(\mathrm{dd}, \mathrm{J}=11.4,2.5 \mathrm{~Hz}, 1 \mathrm{H}), 2.74-2.54$ $(\mathrm{m}, 2 \mathrm{H}), 2.47(\mathrm{dd}, \mathrm{J}=10.5,2.4 \mathrm{~Hz}, 1 \mathrm{H}), 2.21(\mathrm{t}, \mathrm{J}=10.9 \mathrm{~Hz}, 1 \mathrm{H}), 1.44-1.18(\mathrm{br}, 3 \mathrm{H}), 1.11(\mathrm{t}, \mathrm{J}=$ $7.1 \mathrm{~Hz}, 3 \mathrm{H}), 0.88(\mathrm{~s}, 9 \mathrm{H}) .{ }^{13} \mathrm{C}$ NMR $\left(101 \mathrm{MHz}, \mathrm{CDCl}_{3}\right) \delta 56.65,48.75,43.69,33.73,25.69,11.59$.

ESI-HR calcd for $\mathrm{C}_{8} \mathrm{H}_{21} \mathrm{~N}_{2}{ }^{+}$: 145.1699, found 145.1699.

\section{Details of Optimizaiton}

Supplementary Table 1. Screening of the amine catalyst ${ }^{a}$ 

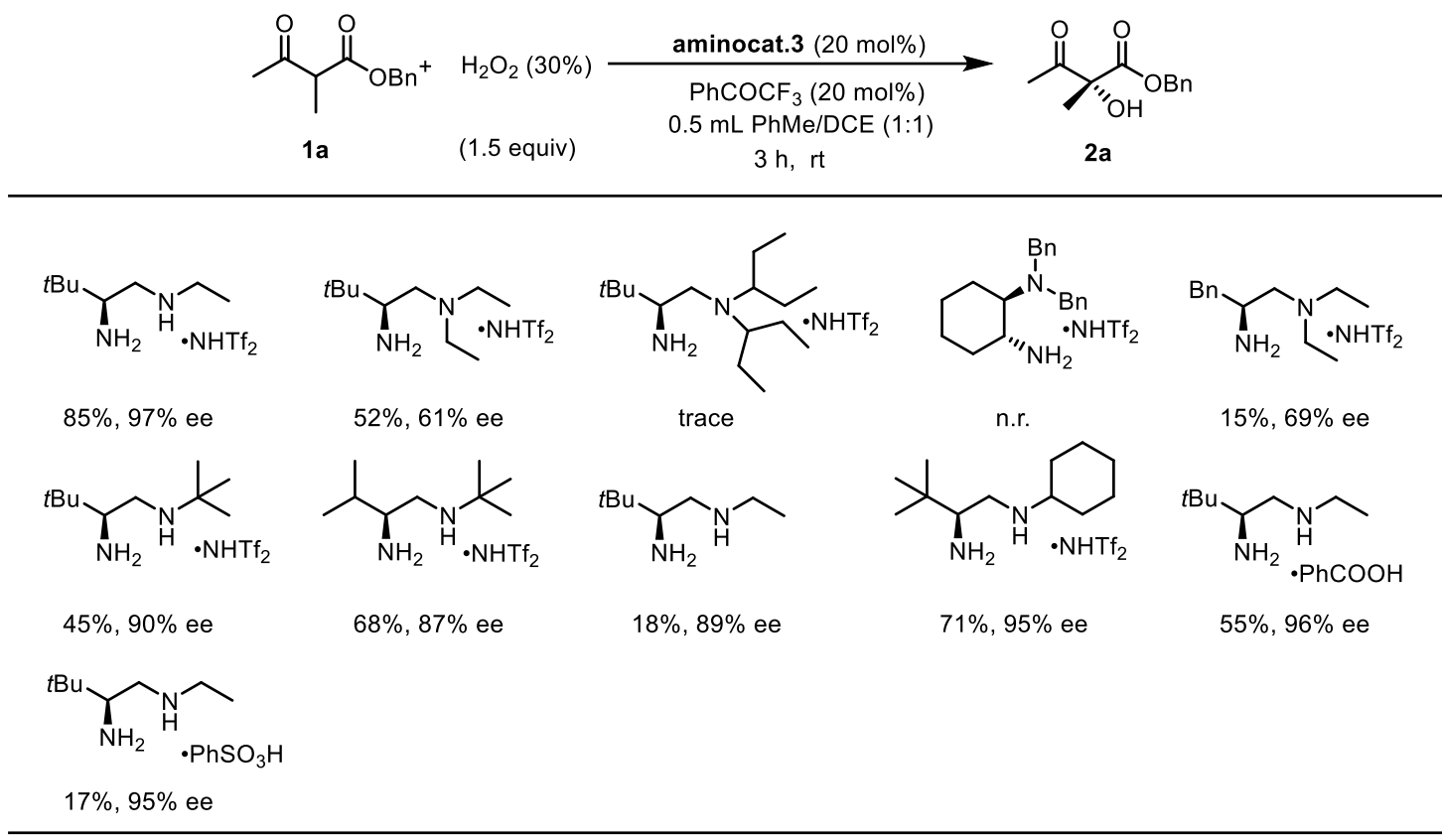

${ }^{a}$ Reactions were performed with $1 \mathrm{a}(0.2 \mathrm{mmol})$, aminocat. $(20 \mathrm{~mol} \%), \mathrm{PhCOCF}_{3}(20 \mathrm{~mol} \%)$, and $\mathrm{H}_{2} \mathrm{O}_{2}$ (30 wt. \% in water, $0.3 \mathrm{mmol}$ ) in $0.5 \mathrm{~mL} \mathrm{PhMe} / \mathrm{DCE}(1 / 1)$ at room temperature in air for $3 \mathrm{~h}$.

Supplementary Table 2. Screening of the oxidants ${ }^{a}$

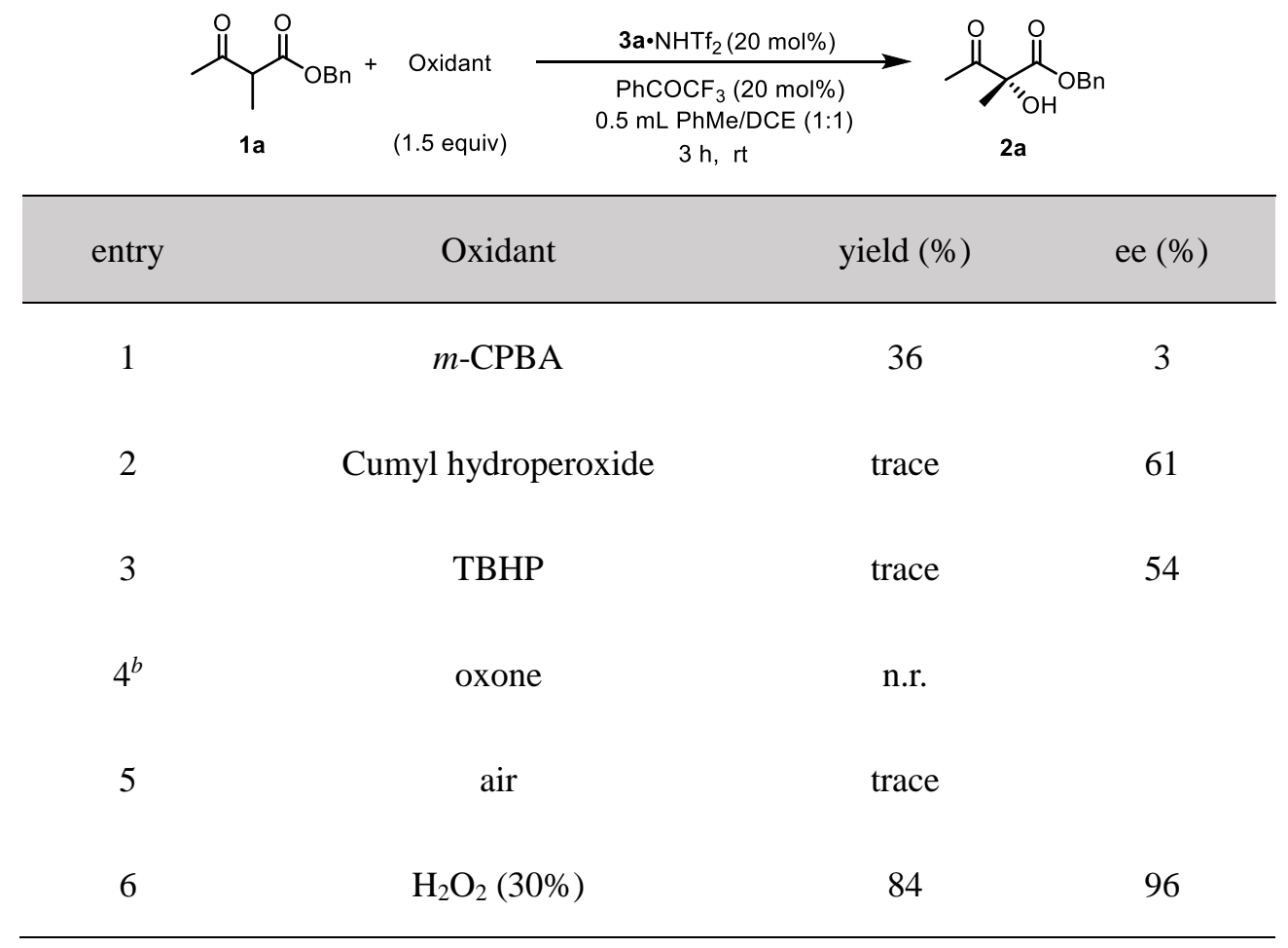

${ }^{a}$ Reactions were performed with $\mathbf{1 a}(0.2 \mathrm{mmol}), 3 \mathrm{a} / \mathrm{NHTf}_{2}(20 \mathrm{~mol} \%), \mathrm{PhCOCF}_{3}(20 \mathrm{~mol} \%)$, and oxidant $(0.3 \mathrm{mmol})$ in $0.5 \mathrm{~mL} \mathrm{PhMe} / \mathrm{DCE}(1 / 1)$ at room temperature in air for $3 \mathrm{~h}$. 
${ }^{b}$ Standard condition with addition of $\mathrm{NaHCO}_{3}(0.6 \mathrm{mmol})$ and aq. Na2 $\mathrm{EDTA}(0.3 \mathrm{~mL}, 4 \times$ $\left.10^{-4} \mathrm{M}\right)$.

Supplementary Table 3. Screening of the solvent ${ }^{a}$

\begin{tabular}{|c|c|c|c|}
\hline entry & Solvent & yield (\%) & ee $(\%)$ \\
\hline 1 & $\mathrm{CH}_{3} \mathrm{CN}$ & 3 & 83 \\
\hline 2 & $\mathrm{Et}_{2} \mathrm{O}$ & 6 & 93 \\
\hline 3 & THF & n.r. & \\
\hline $4^{b}$ & DCM & 45 & 95 \\
\hline 5 & DCE & 65 & 95 \\
\hline 6 & $\mathrm{MeOH}$ & n.r. & \\
\hline 7 & Hexane & 45 & 93 \\
\hline 8 & $\mathrm{PhF}_{6}$ & 22 & 96 \\
\hline 9 & $\mathrm{PhMe}$ & 71 & 95 \\
\hline 10 & $\mathrm{PhMe} / \mathrm{DCE}(1 / 1)$ & 85 & 97 \\
\hline
\end{tabular}

${ }^{a}$ Reactions were performed with 1a $(0.2 \mathrm{mmol}), 3 \mathrm{a} / \mathrm{NHTf}_{2}(20 \mathrm{~mol} \%), \mathrm{PhCOCF}_{3}(20 \mathrm{~mol} \%)$, and $\mathrm{H}_{2} \mathrm{O}_{2}(30 \%$ wt. $\%, 0.3 \mathrm{mmol})$ in $0.5 \mathrm{~mL}$ solvent at room temperature in air for $3 \mathrm{~h}$.

Supplementary Table 4. Screening of the ketone catalyst $t^{a}$

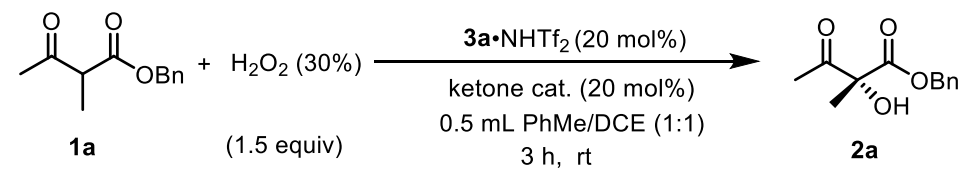




\begin{tabular}{|c|c|c|c|}
\hline entry & ketone cat. & yield (\%) & ee $(\%)$ \\
\hline 1 & -- & 27 & 95 \\
\hline $2^{b}$ & -- & 81 & 96 \\
\hline 3 & $4-\mathrm{NO}_{2} \mathrm{PhCHO}$ & trace & \\
\hline 4 & Pentafluorobenzaldehyde & trace & \\
\hline 5 & $\mathrm{CH}_{3} \mathrm{COCH}_{3}$ & 6 & 92 \\
\hline 6 & $4^{\prime}-\mathrm{CF}_{3} \mathrm{PhCOCH}_{3}$ & 26 & 95 \\
\hline 7 & $\mathrm{CF}_{3} \mathrm{COCH}_{3}$ & 81 & 96 \\
\hline 8 & $\mathrm{PhCOCF}_{3}$ & 85 & 97 \\
\hline 9 & $\mathrm{CF}_{3} \mathrm{COCF}_{3} \cdot \mathrm{H}_{2} \mathrm{O}$ & 86 & 94 \\
\hline 10 & PhCOCOOMe & 86 & 95 \\
\hline 11 & $4 d$ & 47 & 95 \\
\hline
\end{tabular}

${ }^{a}$ Reactions were performed with $\mathbf{1 a}(0.2 \mathrm{mmol}), \mathbf{3 a} / \mathrm{NHTf}_{2}(20 \mathrm{~mol} \%)$, ketone catalyst (20 mol\%), and $\mathrm{H}_{2} \mathrm{O}_{2}$ (30\% wt. $\left.\%, 0.3 \mathrm{mmol}\right)$ in $0.5 \mathrm{~mL} \mathrm{PhMe} / \mathrm{DCE}(1 / 1)$ at room temperature in air for $3 \mathrm{~h} .{ }^{b}$ Reaction time was prolonged to $48 \mathrm{~h}$.

Supplementary Table 5. Screening of the Additives ${ }^{a}$

\begin{tabular}{|c|c|c|c|c|}
\hline & $\begin{array}{l}\mathrm{H}_{2} \mathrm{O}_{2}(30 \%) \\
\text { (1.5 equiv) }\end{array}$ & $\begin{array}{c}\text { 3a-NHTf } \\
2(20 \mathrm{~mol} \%) \\
\mathrm{PhCOCF}_{3}(20 \mathrm{~mol} \%) \\
\text { additives }(20 \mathrm{~mol} \%) \\
0.5 \mathrm{~mL} \text { PhMe/DCE }(1: 1 \\
3 \mathrm{~h}, \mathrm{rt}\end{array}$ & 1) & \\
\hline entry & additives & & yield (\%) & ee $(\%)$ \\
\hline 1 & -- & & 85 & 97 \\
\hline 2 & $\mathrm{NaHCO}_{3}$ & & 55 & 96 \\
\hline
\end{tabular}


${ }^{a}$ Reactions were performed with $\mathbf{1 a}(0.2 \mathrm{mmol}), 3 \mathbf{3 a} / \mathrm{NHTf}_{2}(20 \mathrm{~mol} \%), \mathrm{PhCOCF}_{3}(20 \mathrm{~mol} \%)$, additives (20 mol\%) and $\mathrm{H}_{2} \mathrm{O}_{2}(30 \mathrm{wt} . \%$ in water, $0.3 \mathrm{mmol})$ in $0.5 \mathrm{~mL} \mathrm{PhMe} / \mathrm{DCE}(1 / 1)$ at room temperature in air for $3 \mathrm{~h}$.

\section{4. $\alpha$-Hydroxylation Reactions and Compound Characterization}

\section{General procedure for $\alpha$-Hydroxylation Reactions:}<smiles>[R]C(=O)C1=[R]CCC[R]1=O</smiles>

$0.2 \mathrm{mmol}$<smiles>[13CH3][18OH]</smiles>

1.5 equiv

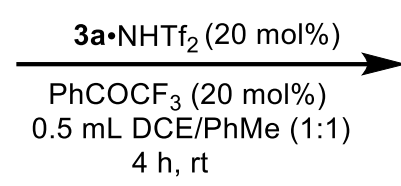
$4 \mathrm{~h}, \mathrm{rt}$

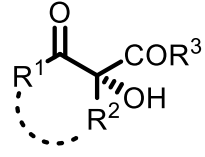

An oven-dried tube equipped with stir bar was charged with corresponding $\beta$-ketocarbonyls $(1,0.2$ mmol) and aminocatalyst 3a/NHTf 2 (20 mol\%). After dissolution in mixed solvent of toluene and 1,2-dichloroethane $(0.5 \mathrm{~mL})$, trifluoroacetophenone $4 \mathbf{a}(20 \mathrm{~mol} \%)$ was added to the vial. Then $\mathrm{H}_{2} \mathrm{O}_{2}$ (30 wt.\% in water, $0.3 \mathrm{mmol}$ ) was added via syringe. Upon completion of the addition, the reaction was conducted at room temperature for at least 4 hours (TLC analysis). The solvent was removed under reduced pressure and the residue was purified by silica gel chromatography with the utilization of ether/hexane or ethyl acetate/hexane to give product 2 . The enantiomeric excess was determined by HPLC. The enantiomeric excess of $\mathbf{2 b - 2 f}$ was determined after benzoylation. 


\section{Characterization of Compounds}

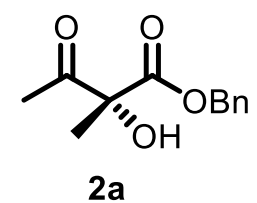

2a: Yellowish oil; Yield: $84 \%$; Enantiomeric excess: $96 \%$; $[\alpha]_{\mathrm{D}}{ }^{24}=51.80\left(\mathrm{c}=1.20, \mathrm{CHCl}_{3}\right)$. HPLC analysis: Daicel Chiralpak As- $\mathrm{H}^{* 2}$, hexane/iso-propanol $=95: 5$, flow rate $=0.8 \mathrm{~mL} / \mathrm{min}$, $\lambda=206 \mathrm{~nm}$, retention time: $18.54 \mathrm{~min}$ (minor) and $19.25 \mathrm{~min}$ (major). ${ }^{1} \mathbf{H} \mathbf{N M R}\left(400 \mathrm{MHz}, \mathrm{CDCl}_{3}\right)$ $\delta 7.45-7.29(\mathrm{~m}, 5 \mathrm{H}), 5.20 \& 5.24(\mathrm{ABq}, 12.3 \mathrm{~Hz}, 2 \mathrm{H}), 4.19(\mathrm{~s}, 1 \mathrm{H}), 2.20(\mathrm{~s}, 3 \mathrm{H}), 1.60(\mathrm{~s}, 3 \mathrm{H})$. ${ }^{13} \mathrm{C}$ NMR $\left(101 \mathrm{MHz}, \mathrm{CDCl}_{3}\right) \delta 204.7,171.2,134.9,128.8,128.8,128.4,81.2,68.2,24.2,21.9$. All data are in accordance with the literature ${ }^{10}$.<smiles>CCOC(=O)[C@](C)(O)C(C)=O</smiles>

2b

2b: Colorless oil; Yield: 90\%; Enantiomeric excess: 95\%; $[\alpha]_{\mathrm{D}}{ }^{24}=45.75\left(\mathrm{c}=0.91, \mathrm{CHCl}_{3}\right)$. HPLC analysis: Daicel Chiralpak OJ-H, hexane/iso-propanol $=90: 10$, flow rate $=1.0 \mathrm{~mL} / \mathrm{min}, \lambda=$ $230 \mathrm{~nm}$, retention time: $13.07 \mathrm{~min}$ (major) and $18.88 \mathrm{~min}$ (minor). ${ }^{1} \mathbf{H} \mathbf{~ N M R}\left(400 \mathrm{MHz}, \mathrm{CDCl}_{3}\right) \delta$ $4.25(\mathrm{q}, J=7.1 \mathrm{~Hz}, 2 \mathrm{H}), 4.15(\mathrm{~s}, 1 \mathrm{H}), 2.27(\mathrm{~s}, 3 \mathrm{H}), 1.58(\mathrm{~s}, 3 \mathrm{H}), 1.29(\mathrm{t}, J=7.1 \mathrm{~Hz}, 3 \mathrm{H}) .{ }^{13} \mathrm{C}$ NMR $\left(101 \mathrm{MHz}, \mathrm{CDCl}_{3}\right) \delta 205.0,171.5,81.1,62.8,24.3,21.9,14.1$. All data are in accordance with the literature. ${ }^{11}$

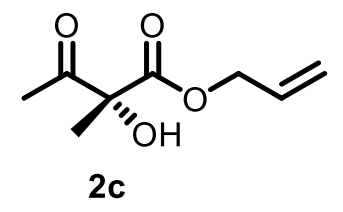

2c: Colorless oil; Yield: 84\%; Enantiomeric excess: $94 \%$; $[\alpha]_{\mathrm{D}}^{24}=59.41\left(\mathrm{c}=1.02, \mathrm{CHCl}_{3}\right) . \mathbf{H P L C}$ analysis: Daicel Chiralpak OJ-H, hexane/iso-propanol $=90: 10$, flow rate $=1.0 \mathrm{~mL} / \mathrm{min}, \lambda=230$ 
nm, retention time: $15.21 \mathrm{~min}$ (major) and $19.74 \mathrm{~min}$ (minor). ${ }^{1} \mathbf{H} \mathbf{N M R}\left(400 \mathrm{MHz}, \mathrm{CDCl}_{3}\right) \delta 5.89$ (ddt, $J=16.4,10.8,5.8 \mathrm{~Hz}, 1 \mathrm{H}), 5.30(\mathrm{~m}, 2 \mathrm{H}), 4.67(\mathrm{~d}, J=5.7 \mathrm{~Hz}, 2 \mathrm{H}), 4.18(\mathrm{~s}, 1 \mathrm{H}), 2.27(\mathrm{~s}, 3 \mathrm{H})$, 1.60 (s, 3H). ${ }^{13}$ C NMR $\left(101 \mathrm{MHz}, \mathrm{CDCl}_{3}\right) \delta 204.8,171.1,131.0,119.6,81.2,67.0,24.3,22.0$. All data are in accordance with the literature. ${ }^{12}$

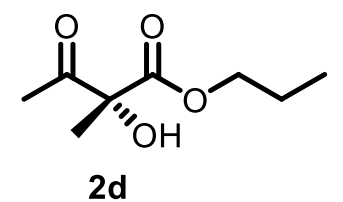

2d: Colorless oil; Yield: 87\%; Enantiomeric excess: 95\%; $[\alpha]_{\mathrm{D}}^{24}=35.08\left(\mathrm{c}=1.28, \mathrm{CHCl}_{3}\right)$. HPLC analysis: Daicel Chiralpak OJ-H, hexane/iso-propanol $=90: 10$, flow rate $=1.0 \mathrm{~mL} / \mathrm{min}, \lambda=$ $230 \mathrm{~nm}$, retention time: $10.84 \mathrm{~min}$ (major) and $14.70 \mathrm{~min}$ (minor). ${ }^{1} \mathbf{H} \mathbf{~ N M R}\left(400 \mathrm{MHz}, \mathrm{CDCl}_{3}\right) \delta$ $4.25-4.06$ (m, 3H), 2.27 (s, 3H), 1.67 (m, 2H), $1.58(\mathrm{~s}, 3 \mathrm{H}), 0.93$ (t, J = $7.4 \mathrm{~Hz}, 3 \mathrm{H}) .{ }^{13} \mathrm{C} \mathrm{NMR}$ $\left(101 \mathrm{MHz}, \mathrm{CDCl}_{3}\right) \delta 204.9,171.6,81.1,68.2,24.3,22.0,21.9,10.3$.

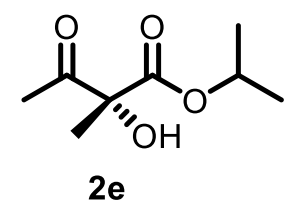

2e: Colorless oil; Yield: $80 \%$; Enantiomeric excess: $93 \%$; $[\alpha]_{\mathrm{D}}{ }^{24}=25.47\left(\mathrm{c}=0.81, \mathrm{CHCl}_{3}\right)$. HPLC analysis: Daicel Chiralpak OJ-H, hexane/iso-propanol $=90: 10$, flow rate $=1.0 \mathrm{~mL} / \mathrm{min}, \lambda=230$ nm, retention time: 8.57 min (major) and $10.52 \mathrm{~min}$ (minor). $\left.{ }^{1} \mathbf{H} \mathbf{~ N M R ~ ( 4 0 0 ~ M H z , ~} \mathrm{CDCl}_{3}\right) \delta 5.09$ (sep, J = 6.3 Hz, 1H), $4.12(\mathrm{~s}, 1 \mathrm{H}), 2.26(\mathrm{~s}, 3 \mathrm{H}), 1.57(\mathrm{~s}, 3 \mathrm{H}), 1.27(\mathrm{~d}, \mathrm{~J}=6.3 \mathrm{~Hz}, 6 \mathrm{H}) .{ }^{13} \mathrm{C}$ NMR $\left(101 \mathrm{MHz}, \mathrm{CDCl}_{3}\right) \delta 204.8,171.1,81.0,70.7,24.3,21.7,21.6,21.6$.

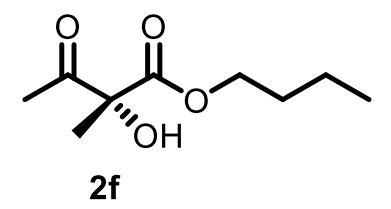

2f: Colorless oil; Yield: 87\%; Enantiomeric excess: $94 \%$; $[\alpha]_{\mathrm{D}}{ }^{24}=35.98\left(\mathrm{c}=1.17, \mathrm{CHCl}_{3}\right)$. HPLC 
analysis: Daicel Chiralpak OJ-H, hexane/iso-propanol $=90: 10$, flow rate $=1.0 \mathrm{~mL} / \mathrm{min}, \lambda=230$ nm, retention time: $8.95 \min$ (major) and $11.71 \mathrm{~min}$ (minor). ${ }^{1} \mathbf{H} \mathbf{N M R}\left(400 \mathrm{MHz}, \mathrm{CDCl}_{3}\right) \delta 4.23$ $-4.17(\mathrm{~m}, 2 \mathrm{H}), 4.15(\mathrm{~s}, 1 \mathrm{H}), 2.27(\mathrm{~s}, 3 \mathrm{H}), 1.68-1.60(\mathrm{~m}, 2 \mathrm{H}), 1.59(\mathrm{~s}, 3 \mathrm{H}), 1.42-1.31(\mathrm{~m}, 2 \mathrm{H})$, $0.93(\mathrm{~m}, 3 \mathrm{H}) .{ }^{13} \mathbf{C}$ NMR $\left(101 \mathrm{MHz}, \mathrm{CDCl}_{3}\right) \delta$ 204.9, 171.6, 81.1, 66.6, 30.6, 24.3, 21.9, 19.1, 13.7.

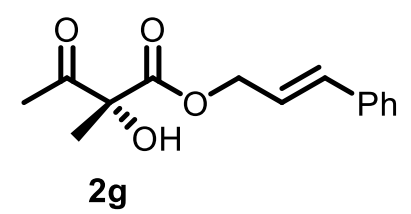

2g: Colorless oil; Yield: 69\%; Enantiomeric excess: 98\%; $[\alpha]_{\mathrm{D}}^{24}=24.83\left(\mathrm{c}=1.18, \mathrm{CHCl}_{3}\right)$. HPLC analysis: Daicel Chiralpak AD-H, hexane/iso-propanol $=98: 2$, flow rate $=1.0 \mathrm{~mL} / \mathrm{min}, \lambda=$ $250 \mathrm{~nm}$, retention time: $46.30 \mathrm{~min}$ (major) and $49.99 \min$ (minor). ${ }^{1} \mathbf{H} \mathbf{~ N M R}\left(400 \mathrm{MHz}, \mathrm{CDCl}_{3}\right) \delta$ $7.41-7.24(\mathrm{~m}, 5 \mathrm{H}), 6.67(\mathrm{~d}, J=15.8 \mathrm{~Hz}, 1 \mathrm{H}), 6.25(\mathrm{dt}, J=15.8,6.6 \mathrm{~Hz}, 1 \mathrm{H}), 4.84(\mathrm{~d}, J=6.5 \mathrm{~Hz}$, 2H), $4.21(\mathrm{~s}, 1 \mathrm{H}), 2.28(\mathrm{~s}, 3 \mathrm{H}), 1.62(\mathrm{~s}, 3 \mathrm{H}) .{ }^{13} \mathrm{C} \mathbf{N M R}\left(101 \mathrm{MHz}, \mathrm{CDCl}_{3}\right) \delta$ 204.9, 171.2, 135.9, 135.7, 128.8, 128.5, 126.8, 121.8, 81.2, 67.1, 24.3, 22.0. ESI-HR calcd for $\mathrm{C}_{14} \mathrm{H}_{16} \mathrm{O}_{4} \mathrm{Na}^{+}$: 271.0941, found 271.0936. IR (thin film, $\mathrm{cm}^{-1}$ ) 3055, 2997, 1722, 1422, 1360, 1264, 1156, 970 , $896,731,703$.

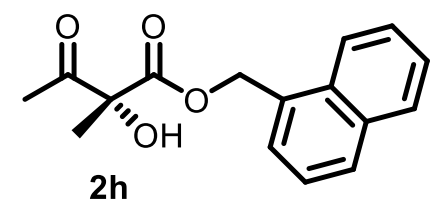

2h: Colorless oil; Yield: 87\%; Enantiomeric excess: 97\%; $[\alpha]_{\mathrm{D}}^{20}=32.99\left(\mathrm{c}=1.97, \mathrm{CHCl}_{3}\right)$. HPLC analysis: Daicel Chiralpak AS-H*2, hexane/iso-propanol $=95: 5$, flow rate $=0.8 \mathrm{~mL} / \mathrm{min}$, $\lambda=223 \mathrm{~nm}$, retention time: $33.58 \mathrm{~min}$ (minor) and $38.36 \mathrm{~min}$ (major). ${ }^{1} \mathbf{H} \mathbf{N M R}\left(400 \mathrm{MHz}, \mathrm{CDCl}_{3}\right)$ $\delta 7.96(\mathrm{~d}, J=8.2 \mathrm{~Hz}, 1 \mathrm{H}), 7.90-7.86(\mathrm{~m}, 2 \mathrm{H}), 7.59-7.50(\mathrm{~m}, 3 \mathrm{H}), 7.48-7.42(\mathrm{~m}, 1 \mathrm{H}), 5.71 \&$ 
$5.66(\mathrm{ABq}, J=12.4 \mathrm{~Hz}, 2 \mathrm{H}), 4.19(\mathrm{~s}, 1 \mathrm{H}), 2.11(\mathrm{~s}, 3 \mathrm{H}), 1.58(\mathrm{~s}, 3 \mathrm{H}) .{ }^{13} \mathrm{C}$ NMR $(101 \mathrm{MHz}$, $\left.\mathrm{CDCl}_{3}\right) \delta 204.7,171.2,133.9,131.6,130.4,129.9,128.9,128.0,126.9,126.2,125.3,123.3,81.2$ 66.6, 24.2, 21.8. ESI-HR calcd for $\mathrm{C}_{16} \mathrm{H}_{16} \mathrm{O}_{4} \mathrm{Na}^{+}$: 295.0941, found 295.0932. IR (thin film, $\mathrm{cm}^{-1}$ ) 3019, 1719, 1214, 1153, 744, 668.<smiles>CC(=O)C(O)(CBr)C(=O)OCc1ccccc1</smiles>

2i: Colorless oil; Yield: 71\%; Enantiomeric excess: $98 \% ;[\alpha]_{\mathrm{D}}{ }^{24}=38.00\left(\mathrm{c}=1.05, \mathrm{CHCl}_{3}\right)$. HPLC analysis: Daicel Chiralpak AS-H, hexane/iso-propanol $=98: 2$, flow rate $=1.0 \mathrm{~mL} / \mathrm{min}, \lambda=208 \mathrm{~nm}$, retention time: $13.94 \mathrm{~min}$ (minor) and $14.60 \mathrm{~min}$ (major). ${ }^{1} \mathbf{H} \mathbf{~ N M R}\left(400 \mathrm{MHz}, \mathrm{CDCl}_{3}\right) \delta 7.44-$ $7.29(\mathrm{~m}, 5 \mathrm{H}), 5.22(\mathrm{ABq}, J=12.2 \mathrm{~Hz}, 2 \mathrm{H}), 4.16(\mathrm{~s}, 1 \mathrm{H}), 2.19(\mathrm{~s}, 3 \mathrm{H}), 2.13(\mathrm{dq}, J=14.8,7.4 \mathrm{~Hz}$, $1 \mathrm{H}), 1.97(\mathrm{dq}, J=14.6,7.4 \mathrm{~Hz}, 1 \mathrm{H}), 0.85(\mathrm{t}, J=7.4 \mathrm{~Hz}, 3 \mathrm{H}) .{ }^{13} \mathrm{C} \mathrm{NMR}\left(101 \mathrm{MHz}, \mathrm{CDCl}_{3}\right) \delta$ 204.9, 170.8, 135.0, 128.8, 128.8, 128.4, 84.7, 68.1, 28.5, 24.7, 7.5. ESI-HR calcd for $\mathrm{C}_{13} \mathrm{H}_{16} \mathrm{O}_{4} \mathrm{Na}^{+}$: 259.0941, found 259.0926. IR (thin film, $\mathrm{cm}^{-1}$ ) 3020, 2997, 1721, 1422, 1214, 1154 , $744,668$.

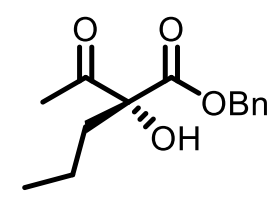

2j

2j: Colorless oil; Yield: 70\%; Enantiomeric excess: 97\%; $[\alpha]_{\mathrm{D}}{ }^{24}=39.21\left(\mathrm{c}=1.40, \mathrm{CHCl}_{3}\right)$. HPLC analysis: Daicel Chiralpak AS-H, hexane/iso-propanol $=98: 2$, flow rate $=0.8 \mathrm{~mL} / \mathrm{min}, \lambda=208 \mathrm{~nm}$, retention time: $16.46 \mathrm{~min}$ (minor) and $17.61 \mathrm{~min}$ (major). ${ }^{1} \mathbf{H} \mathbf{N M R}\left(400 \mathrm{MHz}, \mathrm{CDCl}_{3}\right) \delta 7.34(\mathrm{~m}$, 5H), $5.21(\mathrm{ABq}, 12.3 \mathrm{~Hz}, 2 \mathrm{H}), 4.16(\mathrm{~s}, 1 \mathrm{H}), 2.20(\mathrm{~s}, 3 \mathrm{H}), 2.07$ (ddd, $J=14.0,10.4,6.2 \mathrm{~Hz}, 1 \mathrm{H})$, 
$1.89(\mathrm{ddd}, J=14.0,10.1,6.4 \mathrm{~Hz}, 1 \mathrm{H}), 1.27(\mathrm{~m}, 2 \mathrm{H}), 0.90(\mathrm{t}, J=7.4 \mathrm{~Hz}, 3 \mathrm{H}) .{ }^{13} \mathbf{C} \mathbf{N M R}(101 \mathrm{MHz}$, $\left.\mathrm{CDCl}_{3}\right) \delta 204.9,170.9,135.0,128.8,128.8,128.4,84.5,68.1,37.4,24.7,16.6,14.2$. ESI-HR calcd for $\mathrm{C}_{14} \mathrm{H}_{18} \mathrm{O}_{4} \mathrm{Na}^{+}:$273.1097, found 273.1089. IR (thin film, $\mathrm{cm}^{-1}$ ) 3019, 2997, 1718, 1214, $1045,744,668$.

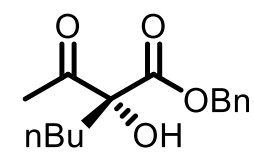

2k

2k: Colorless oil; Yield: 94\%; Enantiomeric excess: 97\%; $[\alpha]_{\mathrm{D}}{ }^{24}=30.27\left(\mathrm{c}=1.10, \mathrm{CHCl}_{3}\right)$. HPLC analysis: Daicel Chiralpak AS-H, hexane/iso-propanol $=98: 2$, flow rate $=0.8 \mathrm{~mL} / \mathrm{min}, \lambda=$ $208 \mathrm{~nm}$, retention time: $14.94 \mathrm{~min}$ (minor) and $16.28 \mathrm{~min}$ (major). ${ }^{1} \mathbf{H} \mathbf{~ N M R}\left(400 \mathrm{MHz}, \mathrm{CDCl}_{3}\right) \delta$ $7.34(\mathrm{~m}, 5 \mathrm{H}), 5.22(\mathrm{ABq}, J=12.2 \mathrm{~Hz}, 2 \mathrm{H}), 4.15(\mathrm{~s}, 1 \mathrm{H}), 2.20(\mathrm{~s}, 3 \mathrm{H}), 2.08$ (ddd, $J=15.0,13.0$, $6.9 \mathrm{~Hz}, 1 \mathrm{H}), 1.91(\mathrm{ddd}, J=15.0,13.0,6.9 \mathrm{~Hz}, 1 \mathrm{H}), 1.34-1.15(\mathrm{~m}, 4 \mathrm{H}), 0.86(\mathrm{t}, J=7.2 \mathrm{~Hz}, 3 \mathrm{H})$. ${ }^{13}$ C NMR (101 MHz, $\left.\mathrm{CDCl}_{3}\right) \delta 204.9,170.9,135.0,128.77,128.75,128.4,84.4,68.1,35.0,25.3$, 24.7, 22.8, 13.9. ESI-HR calcd for $\mathrm{C}_{15} \mathrm{H}_{20} \mathrm{O}_{4} \mathrm{Na}^{+}$: 287.1254, found 287.1246. 273.1097, found 273.1089. IR (thin film, $\mathrm{cm}^{-1}$ ) 3020, 2997, 1718, 1214, 1152, 1047, 744, 668.

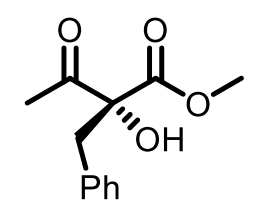

2l

21: Colorless oil; Yield: 77\%; Enantiomeric excess: $96 \%$; $[\alpha]_{\mathrm{D}}{ }^{24}=-13.09\left(\mathrm{c}=1.36, \mathrm{CHCl}_{3}\right)$. HPLC analysis: Daicel Chiralpak AS-H, hexane/iso-propanol $=95: 5$, flow rate $=0.8 \mathrm{~mL} / \mathrm{min}, \lambda=$ $209 \mathrm{~nm}$, retention time: $14.56 \mathrm{~min}$ (minor) and $16.05 \mathrm{~min}$ (major). ${ }^{1} \mathbf{H} \mathbf{~ N M R}\left(400 \mathrm{MHz}, \mathrm{CDCl}_{3}\right) \delta$ $7.30-7.19(\mathrm{~m}, 5 \mathrm{H}), 4.06(\mathrm{~s}, 1 \mathrm{H}), 3.78(\mathrm{~s}, 3 \mathrm{H}), 3.43(\mathrm{~d}, J=14.0 \mathrm{~Hz}, 1 \mathrm{H}), 3.18(\mathrm{~d}, J=14.0 \mathrm{~Hz}$, 
1H), 2.29 (s, 3H). ${ }^{13}$ C NMR (101 MHz, $\left.\mathrm{CDCl}_{3}\right) \delta 204.1,171.1,134.6,130.2,128.4,127.4,84.6$, 53.6, 41.2, 25.4. ESI-HR calcd for $\mathrm{C}_{12} \mathrm{H}_{14} \mathrm{O}_{4} \mathrm{Na}^{+}: 245.0784$, found 245.0777. IR (thin film, $\mathrm{cm}^{-1}$ ) 3495, 3056, 2956, 1720, 1604, 1496, 1455, 1438, 1265, 1122, 733, 702.

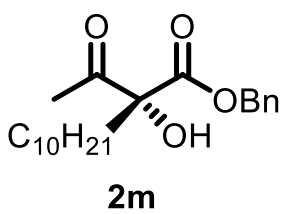

2m: Colorless oil; Yield: 77\%; Enantiomeric excess: 97\%; $[\alpha]_{\mathrm{D}}^{20}=17.51\left(\mathrm{c}=2.51 \mathrm{CHCl}_{3}\right)$. HPLC analysis: Daicel Chiralpak AS- $\mathrm{H}^{* 2}$, hexane/iso-propanol $=95: 5$, flow rate $=0.8 \mathrm{~mL} / \mathrm{min}$, $\lambda=209 \mathrm{~nm}$, retention time: $16.45 \min$ (minor) and $17.36 \mathrm{~min}$ (major). ${ }^{1} \mathbf{H} \mathbf{N M R}\left(400 \mathrm{MHz}, \mathrm{CDCl}_{3}\right)$ $\delta 7.51-7.28(\mathrm{~m}, 5 \mathrm{H}), 5.22(\mathrm{ABq}, J=12.2 \mathrm{~Hz}, 2 \mathrm{H}), 4.15(\mathrm{~s}, 1 \mathrm{H}), 2.21(\mathrm{~s}, 3 \mathrm{H}), 2.08(\mathrm{dt}, J=15.0$, 7.0 Hz, 1H), $1.90(\mathrm{dt}, J=14.1,6.9 \mathrm{~Hz}, 1 \mathrm{H}), 1.23(\mathrm{~m}, 16 \mathrm{H}), 0.88(\mathrm{t}, J=6.8 \mathrm{~Hz}, 3 \mathrm{H}) .{ }^{13} \mathbf{C}$ NMR $\left(101 \mathrm{MHz}, \mathrm{CDCl}_{3}\right) \delta 205.0,170.9,135.0,128.8,128.8,128.5,84.5,68.1,35.3,32.0,29.7,29.7$, 29.6, 29.5, 29.4, 24.8, 23.2, 22.8, 14.3. ESI-HR calcd for $\mathrm{C}_{21} \mathrm{H}_{32} \mathrm{O}_{4} \mathrm{Na}^{+}$: 371.2193, found 371.2181. IR (thin film, $\mathrm{cm}^{-1}$ ) 3020, 2927, 2856, 1718, 1457, 1360, 1214, 1144, 745, 698, 668.<smiles>CC(=O)[C@](O)(CC#N)C(=O)OBr</smiles>

2n

2n: Colorless oil; Yield: 70\%; Enantiomeric excess: $96 \%$; $[\alpha]_{\mathrm{D}}^{24}=-50.00\left(\mathrm{c}=1.48, \mathrm{CHCl}_{3}\right)$. HPLC analysis: Daicel Chiralpak AS-H, hexane/iso-propanol $=90: 10$, flow rate $=1.0 \mathrm{~mL} / \mathrm{min}, \lambda=$ $209 \mathrm{~nm}$, retention time: $14.37 \mathrm{~min}$ (major) and $16.81 \mathrm{~min}$ (minor). ${ }^{1} \mathbf{H} \mathbf{~ N M R}\left(400 \mathrm{MHz}, \mathrm{CDCl}_{3}\right) \delta$ $7.44-7.29$ (m, 5H), 5.29 (s, 2H), $4.48(\mathrm{~s}, 1 \mathrm{H}), 3.11(\mathrm{~d}, J=17.0 \mathrm{~Hz}, 1 \mathrm{H}), 2.96(\mathrm{~d}, J=17.0 \mathrm{~Hz}$, 1H), 2.18 (s, 3H). ${ }^{13} \mathbf{C}$ NMR (101 MHz, $\left.\mathrm{CDCl}_{3}\right) \delta$ 200.5, 168.8, 133.9, 129.3, 129.0, 128.7, 115.4, 
80.7, 69.8, 24.7, 24.3. ESI-HR calcd for $\mathrm{C}_{13} \mathrm{H}_{13} \mathrm{NO}_{4} \mathrm{Na}^{+}: 270.0737$, found 270.0729. IR (thin film, $\left.\mathrm{cm}^{-1}\right) 3020,2400,1731,1421,1214,1135,745,668$

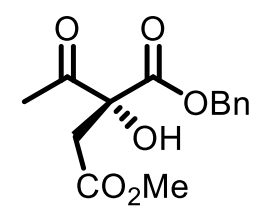

20

2o: Colorless oil; 8 h, Yield: 61\%; Enantiomeric excess: 97\%; $[\alpha]_{\mathrm{D}^{24}}=-14.40\left(\mathrm{c}=1.04, \mathrm{CHCl}_{3}\right)$. HPLC analysis: Daicel Chiralpak OJ-H, hexane/iso-propanol $=90: 10$, flow rate $=1.0 \mathrm{~mL} / \mathrm{min}, \lambda=$ $209 \mathrm{~nm}$, retention time: $24.08 \mathrm{~min}$ (major) and $28.97 \mathrm{~min}$ (minor). ${ }^{\mathbf{1}} \mathbf{H} \mathbf{N M R}\left(400 \mathrm{MHz}, \mathrm{CDCl}_{3}\right) \delta$ 7.36-7.26 (m, 5H), $5.21(\mathrm{ABq}, 12.3 \mathrm{~Hz}, 2 \mathrm{H}), 4.52(\mathrm{~s}, 1 \mathrm{H}), 3.64(\mathrm{~s}, 3 \mathrm{H}), 3.20(\mathrm{~d}, J=16.8 \mathrm{~Hz}, 1 \mathrm{H})$, $3.04(\mathrm{~d}, J=16.8 \mathrm{~Hz}, 1 \mathrm{H}), 2.23(\mathrm{~s}, 3 \mathrm{H}) .{ }^{13} \mathbf{C} \mathbf{~ N M R}\left(101 \mathrm{MHz}, \mathrm{CDCl}_{3}\right) \delta 203.1,170.7,169.8,134.7$, 128.84, 128.80, 128.4, 82.0, 68.7, 52.2, 39.8, 24.6. ESI-HR calcd for $\mathrm{C}_{14} \mathrm{H}_{16} \mathrm{O}_{6} \mathrm{Na}^{+}$: 303.0839 , found 303.0828. IR (thin film, $\mathrm{cm}^{-1}$ ) 3054, 2987, 1729, 1422, 1264, 869, 731, 703.<smiles>CCOC(O)(CC)C(=O)OCc1ccccc1</smiles>

2p: Colorless oil; 12 h, Yield: 53\%; Enantiomeric excess: 96\%; $[\alpha]_{\mathrm{D}}^{20}=-6.17\left(\mathrm{c}=3.41, \mathrm{CHCl}_{3}\right)$. HPLC analysis: Daicel Chiralpak OJ-H, hexane/iso-propanol $=95: 5$, flow rate $=1.0 \mathrm{~mL} / \mathrm{min}, \lambda=$ $208 \mathrm{~nm}$, retention time: $16.11 \mathrm{~min}$ (minor) and $19.45 \mathrm{~min}$ (major). ${ }^{1} \mathbf{H} \mathbf{~ N M R}\left(400 \mathrm{MHz}, \mathrm{CDCl}_{3}\right) \delta$ $7.33(\mathrm{~m}, 5 \mathrm{H}), 5.29-5.13(\mathrm{~m}, 2 \mathrm{H}), 4.57(\mathrm{~s}, 1 \mathrm{H}), 3.10(\mathrm{~d}, J=16.6 \mathrm{~Hz}, 1 \mathrm{H}), 2.96(\mathrm{~d}, J=16.6 \mathrm{~Hz}$ 1H), 2.23 (s, 3H), 1.40 (s, 9H). ${ }^{13} \mathbf{C}$ NMR (101 MHz, $\left.\mathrm{CDCl}_{3}\right) \delta$ 203.4, 169.9, 169.3, 134.9, 128.82, 128.8, 128.4, 82.3, 82.1, 68.4, 41.0, 28.1, 24.7. ESI-HR calcd for $\mathrm{C}_{17} \mathrm{H}_{22} \mathrm{O}_{6} \mathrm{Na}^{+}$: 345.1309, found 345.1296. IR (thin film, $\mathrm{cm}^{-1}$ ) 3020, 2981, 1726, 1456, 1370, 1359, 1214, 1155, 845, 744, 668. 


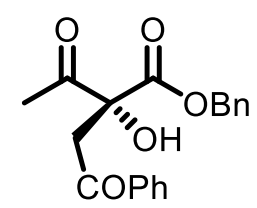

2q

2q: Colorless oil; 7 h, Yield: 70\%; Enantiomeric excess: 98\%; $[\alpha]_{\mathrm{D}}^{20}=-16.38\left(\mathrm{c}=2.6, \mathrm{CHCl}_{3}\right)$. HPLC analysis: Daicel Chiralpak OJ-H, hexane/iso-propanol $=95: 5$, flow rate $=1.0 \mathrm{~mL} / \mathrm{min}, \lambda=$ $242 \mathrm{~nm}$, retention time: $36.87 \mathrm{~min}$ (major) and $40.37 \mathrm{~min}$ (minor). ${ }^{1} \mathbf{H} \mathbf{~ N M R}\left(400 \mathrm{MHz}, \mathrm{CDCl}_{3}\right) \delta$ $8.01-7.85(\mathrm{~m}, 2 \mathrm{H}), 7.58(\mathrm{~m}, 1 \mathrm{H}), 7.46(\mathrm{~m}, 2 \mathrm{H}), 4.61(\mathrm{~s}, 1 \mathrm{H}), 4.27(\mathrm{~m}, 2 \mathrm{H}), 3.91(\mathrm{~d}, \mathrm{~J}=17.9 \mathrm{~Hz}$, 1H), $3.68(\mathrm{~d}, \mathrm{~J}=17.8 \mathrm{~Hz}, 1 \mathrm{H}), 2.35(\mathrm{~s}, 3 \mathrm{H}), 1.26(\mathrm{t}, \mathrm{J}=7.1 \mathrm{~Hz}, 3 \mathrm{H}) .{ }^{13} \mathbf{C} \mathbf{~ N M R}\left(101 \mathrm{MHz}, \mathrm{CDCl}_{3}\right)$ $\delta 204.38,197.51,170.31,136.29,133.95,128.84,128.36,82.40,77.48,77.16,76.84,63.05,43.83$, 24.76, 14.06. ESI-HR calcd for $\mathrm{C}_{14} \mathrm{H}_{16} \mathrm{O}_{5} \mathrm{Na}^{+}$: 287.0890, found 287.0875. IR (thin film, $\mathrm{cm}^{-1}$ ) 3462, 2982, 2930, 1720, 1686, 1597, 1449, 1354, 1219, 1136, 1072, 1013, 856, 756, 689.

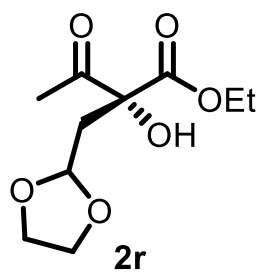

2r: Yellowish oil; 12 h, Yield: 62\%; Enantiomeric excess: $97 \%$; $[\alpha]_{\mathrm{D}}^{20}=-7.11\left(\mathrm{c}=2.11, \mathrm{CHCl}_{3}\right)$. HPLC analysis: Daicel Chiralpak AD-H, hexane/iso-propanol $=95: 5$, flow rate $=0.8 \mathrm{~mL} / \mathrm{min}, \lambda=$ $205 \mathrm{~nm}$, retention time: 20.07 min (major) and $23.35 \mathrm{~min}$ (minor). ${ }^{\mathbf{1}} \mathbf{H} \mathbf{~ N M R}\left(400 \mathrm{MHz}, \mathrm{CDCl}_{3}\right) \delta$ $5.04(\mathrm{dd}, J=5.4,4.2 \mathrm{~Hz}, 1 \mathrm{H}), 4.43(\mathrm{~s}, 1 \mathrm{H}), 4.23(\mathrm{td}, J=7.1,1.7 \mathrm{~Hz}, 2 \mathrm{H}), 3.97-3.86(\mathrm{~m}, 2 \mathrm{H})$, $3.86-3.76(\mathrm{~m}, 2 \mathrm{H}), 2.52(\mathrm{dd}, J=14.6,4.2 \mathrm{~Hz}, 1 \mathrm{H}), 2.33(\mathrm{dd}, J=14.6,5.5 \mathrm{~Hz}, 1 \mathrm{H}), 2.26(\mathrm{~s}, 3 \mathrm{H})$, $1.27(\mathrm{t}, J=7.1 \mathrm{~Hz}, 3 \mathrm{H}) .{ }^{13} \mathrm{C} \mathbf{N M R}\left(101 \mathrm{MHz}, \mathrm{CDCl}_{3}\right) \delta 204.0,170.5,101.4,82.0,65.1,64.9,62.7$, 38.5, 24.7, 14.1. ESI-HR calcd for $\mathrm{C}_{10} \mathrm{H}_{16} \mathrm{O}_{6} \mathrm{Na}^{+}: 255.0839$, found 255.0829. IR (thin film, $\mathrm{cm}^{-1}$ ) 3020, 2981, 1722, 1358, 1214, 1144, 1032. 


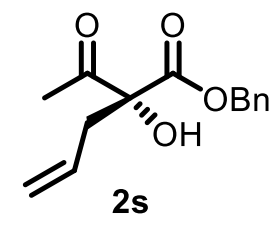

2s: Colorless oil; Yield: $81 \%$; Enantiomeric excess: $98 \%$; $[\alpha]_{\mathrm{D}}^{24}=18.03\left(\mathrm{c}=1.07, \mathrm{CHCl}_{3}\right)$. HPLC analysis: Daicel Chiralpak As- $\mathrm{H}^{*}$, hexane/iso-propanol $=95: 5$, flow rate $=0.8 \mathrm{~mL} / \mathrm{min}, \lambda=208$ nm, retention time: $20.82 \mathrm{~min}$ (minor) and $22.15 \mathrm{~min}$ (major). ${ }^{1} \mathbf{H} \mathbf{~ N M R}\left(400 \mathrm{MHz}, \mathrm{CDCl}_{3}\right) \delta 7.40$ $-7.30(\mathrm{~m}, 5 \mathrm{H}), 5.73-5.61(\mathrm{~m}, 1 \mathrm{H}), 5.21(\mathrm{ABq}, J=12.2 \mathrm{~Hz}, 2 \mathrm{H}), 5.16-5.07(\mathrm{~m}, 2 \mathrm{H}), 4.18(\mathrm{~s}$, 1H), $2.85(\mathrm{dd}, J=14.4,6.7 \mathrm{~Hz}, 1 \mathrm{H}), 2.69(\mathrm{dd}, J=14.4,7.6 \mathrm{~Hz}, 1 \mathrm{H}), 2.19(\mathrm{~s}, 3 \mathrm{H}) .{ }^{13} \mathrm{C}$ NMR $(101$ $\left.\mathrm{MHz}, \mathrm{CDCl}_{3}\right) \delta 204.0,170.4,134.9,130.9,128.8,128.5,120.0,83.9,68.3,39.6,24.9$. ESI-HR calcd for $\mathrm{C}_{14} \mathrm{H}_{16} \mathrm{O}_{4} \mathrm{Na}^{+}$: 271.0941, found 271.0932. IR (thin film, $\mathrm{cm}^{-1}$ ) 3020, 1721, 1540, 744, 668.

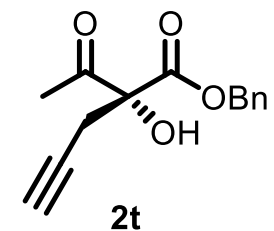

2t: Colorless oil; Yield: 71\%; Enantiomeric excess: $96 \% ;[\alpha]_{\mathrm{D}}^{24}=-18.11\left(\mathrm{c}=1.11, \mathrm{CHCl}_{3}\right)$. HPLC analysis: Daicel Chiralpak As-H, hexane/iso-propanol $=98: 2$, flow rate $=0.8 \mathrm{~mL} / \mathrm{min}, \lambda=$ $208 \mathrm{~nm}$, retention time: $31.55 \mathrm{~min}$ (minor) and $33.87 \mathrm{~min}$ (major). ${ }^{1} \mathbf{H} \mathbf{~ N M R}\left(400 \mathrm{MHz}, \mathrm{CDCl}_{3}\right) \delta$ $7.35(\mathrm{~m}, 5 \mathrm{H}), 5.24(\mathrm{ABq}, J=12.2 \mathrm{~Hz}, 2 \mathrm{H}), 4.36(\mathrm{~s}, 1 \mathrm{H}), 3.00(\mathrm{dd}, J=17.2,2.6 \mathrm{~Hz}, 1 \mathrm{H}), 2.89(\mathrm{dd}$, $J=17.2,2.7 \mathrm{~Hz}, 1 \mathrm{H}), 2.21(\mathrm{~s}, 3 \mathrm{H}), 2.00(\mathrm{t}, J=2.7 \mathrm{~Hz}, 1 \mathrm{H}) .{ }^{13} \mathbf{C} \mathbf{N M R}\left(101 \mathrm{MHz}, \mathrm{CDCl}_{3}\right) \delta 202.3$, $169.5,134.6,128.9,128.8,128.5,82.7,77.7,72.0,68.7,26.0,24.7$. ESI-HR calcd for $\mathrm{C}_{14} \mathrm{H}_{14} \mathrm{O}_{4} \mathrm{Na}^{+}$: 269.0784, found 269.0775. IR (thin film, $\mathrm{cm}^{-1}$ ) 3308, 3019, 1726, 1214, 744, 668. 


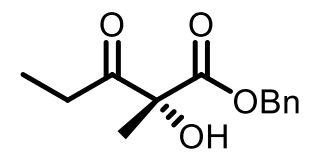

$2 u$

2u: Colorless oil; 12 h, Yield: 59\%; Enantiomeric excess: $96 \% .[\alpha]_{\mathrm{D}}{ }^{24}=32.75\left(\mathrm{c}=0.51, \mathrm{CHCl}_{3}\right)$. HPLC analysis: Daicel Chiralpak OJ-H, hexane/iso-propanol $=95: 5$, flow rate $=1.0 \mathrm{~mL} / \mathrm{min}, \lambda=$ $208 \mathrm{~nm}$, retention time: $15.81 \mathrm{~min}$ (major) and $19.29 \min$ (minor). ${ }^{1} \mathbf{H} \mathbf{~ N M R}\left(400 \mathrm{MHz}, \mathrm{CDCl}_{3}\right) \delta$ $7.42-7.27(\mathrm{~m}, 5 \mathrm{H}), 5.21(\mathrm{ABq}, J=12.2 \mathrm{~Hz}, 2 \mathrm{H}), 4.20(\mathrm{~s}, 1 \mathrm{H}), 2.65-2.40(\mathrm{~m}, 2 \mathrm{H}), 1.60(\mathrm{~s}, 3 \mathrm{H})$, $1.01(\mathrm{t}, J=7.2 \mathrm{~Hz}, 3 \mathrm{H}) .{ }^{13} \mathrm{C}$ NMR $\left(101 \mathrm{MHz}, \mathrm{CDCl}_{3}\right) \delta 207.7,171.4,135.0,128.80,128.76$, 128.3, 81.1, 68.1, 29.9, 22.0, 7.8. ESI-HR calcd for $\mathrm{C}_{13} \mathrm{H}_{16} \mathrm{O}_{4} \mathrm{Na}^{+}:$: 259.0941, found 259.0934. IR (thin film, $\mathrm{cm}^{-1}$ ) 3019, 1721, 1214, 1041, 744, 668.

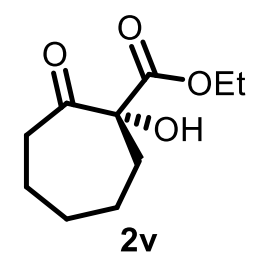

2v: Colorless oil; Yield: 82\%; Enantiomeric excess: $94 \%$; $[\alpha]_{\mathrm{D}}{ }^{24}=137.84\left(\mathrm{c}=0.88, \mathrm{CHCl}_{3}\right)$. HPLC analysis: Daicel Chiralpak OD-H, hexane/iso-propanol $=95: 5$, flow rate $=0.8 \mathrm{~mL} / \mathrm{min}, \lambda=$ $205 \mathrm{~nm}$, retention time: $7.78 \mathrm{~min}$ (minor) and $8.43 \mathrm{~min}$ (major). ${ }^{\mathbf{1}} \mathbf{H} \mathbf{N M R}\left(400 \mathrm{MHz}, \mathrm{CDCl}_{3}\right) \delta$ $4.27(\mathrm{~s}, 1 \mathrm{H}), 4.19(\mathrm{qd}, J=7.1,2.0 \mathrm{~Hz}, 2 \mathrm{H}), 2.97-2.91(\mathrm{~m}, 1 \mathrm{H}), 2.60-2.54(\mathrm{~m}, 1 \mathrm{H}), 2.25(\mathrm{dd}, J=$ $14.9,10.9 \mathrm{~Hz}, 1 \mathrm{H}), 2.09(\mathrm{dd}, J=14.7,8.0 \mathrm{~Hz}, 1 \mathrm{H}), 2.03-1.72(\mathrm{~m}, 3 \mathrm{H}), 1.57-1.39(\mathrm{~m}, 2 \mathrm{H}), 1.38$ - 1.17 (m, 4H). ${ }^{13} \mathbf{C}$ NMR $\left(101 \mathrm{MHz}, \mathrm{CDCl}_{3}\right) \delta 209.7,170.7,83.6,62.3,40.1,34.6,30.3,27.2$, 23.8, 14.1. ESI-HR calcd for $\mathrm{C}_{10} \mathrm{H}_{16} \mathrm{O}_{4} \mathrm{Na}^{+}$: 223.0941, found 223.0932. IR (thin film, $\mathrm{cm}^{-1}$ ) 3020, $1711,1214,1041,744,668$. 


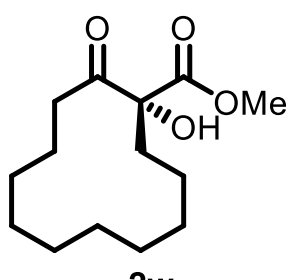

$2 \mathbf{w}$

2w: Colorless oil; Yield: 30\%; Enantiomeric excess: 98\%; $[\alpha]_{\mathrm{D}}^{20}=121.24\left(\mathrm{c}=1.50, \mathrm{CHCl}_{3}\right)$. HPLC analysis: Daicel Chiralpak As-H*2, hexane/iso-propanol $=95: 5$, flow rate $=0.8 \mathrm{~mL} / \mathrm{min}$, $\lambda=205 \mathrm{~nm}$, retention time: $15.31 \mathrm{~min}$ (major) and $16.41 \mathrm{~min}$ (minor). ${ }^{1} \mathbf{H} \mathbf{N M R}\left(400 \mathrm{MHz}, \mathrm{CDCl}_{3}\right.$ ) $\delta 4.37(\mathrm{~s}, 1 \mathrm{H}), 3.76(\mathrm{~s}, 3 \mathrm{H}), 3.18(\mathrm{ddd}, J=18.9,12.5,2.8 \mathrm{~Hz}, 1 \mathrm{H}), 2.34-2.27(\mathrm{~m}, 1 \mathrm{H}), 2.24-$ $2.12(\mathrm{~m}, 2 \mathrm{H}), 2.07-1.98(\mathrm{~m}, 1 \mathrm{H}), 1.54-1.45(\mathrm{~m}, 1 \mathrm{H}), 1.42-1.18(\mathrm{~m}, 13 \mathrm{H}), 0.87-0.70(\mathrm{~m}, 1 \mathrm{H})$. ${ }^{13} \mathrm{C}$ NMR (101 MHz, $\left.\mathrm{CDCl}_{3}\right) \delta$ 208.1, 171.3, 84.7, 53.2, 33.8, 33.3, 26.5, 26.4, 24.0, 23.0, 22.8, 22.7, 21.8, 19.2. ESI-HR calcd for $\mathrm{C}_{14} \mathrm{H}_{24} \mathrm{O}_{4} \mathrm{Na}^{+}: 279.1567$, found 279.1558. IR (thin film, $\mathrm{cm}^{-1}$ ) $3019,1715,1436,1214,1041,744,668$.

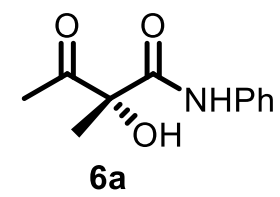

6a: colorless solid; Yield: 74\%; Enantiomeric excess: 99\%; $[\alpha]_{\mathrm{D}}^{20}=129.27\left(\mathrm{c}=2.6, \mathrm{CHCl}_{3}\right)$. HPLC analysis: Daicel Chiralpak As-H, hexane/iso-propanol $=95: 5$, flow rate $=0.8 \mathrm{~mL} / \mathrm{min}, \lambda=$ $206 \mathrm{~nm}$, retention time: $10.80 \mathrm{~min}$ (major) and $12.64 \mathrm{~min}$ (minor). ${ }^{1} \mathbf{H} \mathbf{~ N M R}\left(400 \mathrm{MHz}, \mathrm{CDCl}_{3}\right) \delta$ $8.80(\mathrm{~s}, 1 \mathrm{H}), 7.54(\mathrm{~d}, J=8.0 \mathrm{~Hz}, 2 \mathrm{H}), 7.34(\mathrm{t}, J=7.8 \mathrm{~Hz}, 2 \mathrm{H}), 7.14(\mathrm{t}, J=7.3 \mathrm{~Hz}, 1 \mathrm{H}), 5.00(\mathrm{~s}$, 1H), 2.53 (s, 3H), 1.72 (s, 3H). ${ }^{13} \mathbf{C}$ NMR (101 MHz, $\left.\mathrm{CDCl}_{3}\right) \delta$ 207.7, 168.1, 137.0, 129.2, 125.0, 119.9, 82.3, 24.6, 24.6. ESI-HR calcd for $\mathrm{C}_{11} \mathrm{H}_{13} \mathrm{NO}_{3} \mathrm{Na}^{+}$: 230.0788, found 230.0781. IR (thin film, $\left.\mathrm{cm}^{-1}\right)$ 3382, 3019, 1709, 1687, 1602, 1525,1444, 1214, 1149, 744, 668. 


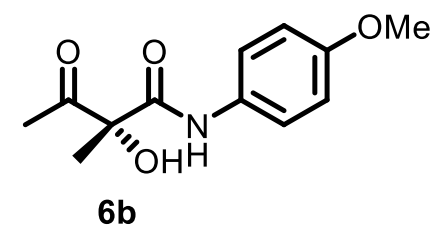

6b: Brown solid; Yield: $65 \%$; Enantiomeric excess: $97 \% ;[\alpha]_{\mathrm{D}}^{20}=177.88\left(\mathrm{c}=3.2, \mathrm{CHCl}_{3}\right)$. HPLC analysis: Daicel Chiralpak OJ-H, hexane/iso-propanol $=90: 10$, flow rate $=1.0 \mathrm{~mL} / \mathrm{min}, \lambda=206$ nm, retention time: 29.36 (minor) and 35.77 min (major). ${ }^{1} \mathbf{H} \mathbf{N M R}\left(400 \mathrm{MHz}, \mathrm{CDCl}_{3}\right) \delta 8.69$ (s, 1H), $7.49-7.38(\mathrm{~m}, 2 \mathrm{H}), 6.91-6.82(\mathrm{~m}, 2 \mathrm{H}), 4.97(\mathrm{~s}, 1 \mathrm{H}), 3.79(\mathrm{~s}, 3 \mathrm{H}), 2.53(\mathrm{~s}, 3 \mathrm{H}), 1.71(\mathrm{~s}, 3 \mathrm{H})$. ${ }^{13}$ C NMR $\left(101 \mathrm{MHz}, \mathrm{CDCl}_{3}\right) \delta 207.9,167.829,156.9,130.2,121.5,114.4,82.2,77.5,77.2,76.8$, 55.6, 24.6, 24.6. ESI-HR calcd for $\mathrm{C}_{12} \mathrm{H}_{15} \mathrm{NO}_{4} \mathrm{Na}^{+}:$260.0893, found 260.0884. IR (thin film, $\mathrm{cm}^{-1}$ ) $3019,1715,1682,1518,1214,1035,744,668$.<smiles>CC(=O)[C@](C)(O)C(=O)Nc1ccccc1Cl</smiles>

6c: Colorless oil; 8 h, Yield: 96\%; Enantiomeric excess: $97 \% ;[\alpha]_{D}{ }^{20}=132.76\left(\mathrm{c}=2.39, \mathrm{CHCl}_{3}\right)$. HPLC analysis: Daicel Chiralpak AD-H, hexane/iso-propanol $=95: 5$, flow rate $=1.0 \mathrm{~mL} / \mathrm{min}, \lambda=$ $208 \mathrm{~nm}$, retention time: 6.79 (major) and $8.11 \mathrm{~min}$ (minor). ${ }^{1} \mathbf{H} \mathbf{N M R}\left(400 \mathrm{MHz}, \mathrm{CDCl}_{3}\right) \delta 9.49(\mathrm{~s}$, $1 \mathrm{H}), 8.36(\mathrm{dd}, J=8.3,1.6 \mathrm{~Hz}, 1 \mathrm{H}), 7.38(\mathrm{dd}, J=8.1,1.6 \mathrm{~Hz}, 1 \mathrm{H}), 7.33-7.22(\mathrm{~m}, 1 \mathrm{H}), 7.07(\mathrm{td}, J$ $=7.8,1.6 \mathrm{~Hz}, 1 \mathrm{H}), 5.07(\mathrm{~s}, 1 \mathrm{H}), 2.54(\mathrm{~s}, 3 \mathrm{H}), 1.74(\mathrm{~s}, 3 \mathrm{H}) .{ }^{13} \mathbf{C} \mathbf{~ N M R}\left(101 \mathrm{MHz}, \mathrm{CDCl}_{3}\right) \delta 207.1$, $168.4,133.9,129.3,127.8,125.3,123.7,121.1,82.6,24.6,24.5$. ESI-HR calcd for $\mathrm{C}_{11} \mathrm{H}_{12} \mathrm{ClNO}_{3} \mathrm{Na}^{+}:$264.0398, found 264.0390. IR (thin film, $\mathrm{cm}^{-1}$ ) 3359, 3019, 1709, 1692, $1525,1214,1036,744,668$. 


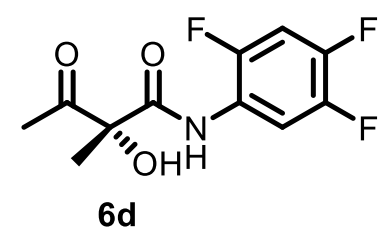

6d: White solid; 8 h, Yield: 95\%; Enantiomeric excess: 98\%; $[\alpha]_{\mathrm{D}}{ }^{20}=134.44\left(\mathrm{c}=2.52, \mathrm{CHCl}_{3}\right)$. HPLC analysis: Daicel Chiralpak OJ-H, hexane/iso-propanol $=90: 10$, flow rate $=1.0 \mathrm{~mL} / \mathrm{min}, \lambda=$ $239 \mathrm{~nm}$, retention time: 7.99 (major) and $8.84 \mathrm{~min}$ (minor). ${ }^{1} \mathbf{H} \mathbf{N M R}\left(400 \mathrm{MHz}, \mathrm{CDCl}_{3}\right) \delta 9.08(\mathrm{~s}$, 1H), $8.28(\mathrm{ddd}, J=12.0,8.4,7.4 \mathrm{~Hz}, 1 \mathrm{H}), 6.98(\mathrm{td}, J=10.0,7.0 \mathrm{~Hz}, 1 \mathrm{H}), 5.01(\mathrm{~s}, 1 \mathrm{H}), 2.52(\mathrm{~s}$, 3H), $1.71(\mathrm{~s}, 3 \mathrm{H}),{ }^{13} \mathrm{C}$ NMR $\left(101 \mathrm{MHz}, \mathrm{CDCl}_{3}\right) \delta 206.6,168.4,147.9(\mathrm{~d}, \mathrm{~J}=243.8 \mathrm{~Hz}), 146.4(\mathrm{~d}$, $\mathrm{J}=256.8 \mathrm{~Hz}), 145.7(\mathrm{~d}, \mathrm{~J}=204.8 \mathrm{~Hz}), 122.0-121.8(\mathrm{~m}), 110.0(\mathrm{~d}, J=24.9 \mathrm{~Hz}), 105.2(\mathrm{dd}, J=$ 25.0, $22.0 \mathrm{~Hz}), 82.4,24.5,24.4 .{ }^{19} \mathbf{F}$ NMR $\left(376 \mathrm{MHz}, \mathrm{CDCl}_{3}\right) \delta-132.14--132.05(\mathrm{~m}, 1 \mathrm{~F})$, $-138.48--138.36(\mathrm{~m}, 1 \mathrm{~F}),-140.23--140.09(\mathrm{~m}, 1 \mathrm{~F})$. ESI-HR calcd for $\mathrm{C}_{11} \mathrm{H}_{10} \mathrm{~F}_{3} \mathrm{NO}_{3} \mathrm{Na}^{+}$: 284.0505, found 284.0496. IR (thin film, $\mathrm{cm}^{-1}$ ) 3381, 3020, 1709, 1694, 1538, 1214, 1046, 877, $744,668$.

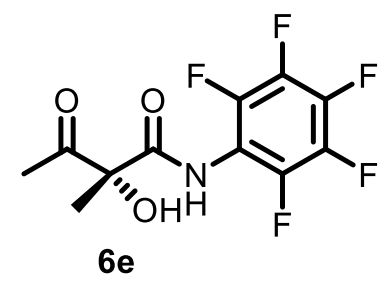

6e: White soild; 24 h, Yield: 79\%; Enantiomeric excess: 97\%; $[\alpha]_{\mathrm{D}^{20}}=161.94\left(\mathrm{c}=1.60, \mathrm{CHCl}_{3}\right)$. HPLC analysis: Daicel Chiralpak OJ-H, hexane/iso-propanol $=95: 5$, flow rate $=1.0 \mathrm{~mL} / \mathrm{min}, \lambda=$ $219 \mathrm{~nm}$, retention time: 16.10 (major) and $18.41 \mathrm{~min}$ (minor). ${ }^{\mathbf{1}} \mathbf{H} \mathbf{~ N M R}\left(400 \mathrm{MHz}, \mathrm{CDCl}_{3}\right) \delta 8.42$ (s, 1H), 4.99 (s, 1H), 2.49 (s, 3H), 1.72 (s, 3H). ${ }^{13} \mathbf{C}$ NMR (101 MHz, $\left.\mathrm{CDCl}_{3}\right) \delta$ 206.3, 169.3, $144.4-144.2(\mathrm{~m}), 141.9-141.7(\mathrm{~m}), 139.42-139.03(\mathrm{~m}), 136.86-136.61(\mathrm{~m}), 111.4-111.1$ (m), 82.4, 24.5, 24.1. ${ }^{19} \mathbf{F}$ NMR $\left(376 \mathrm{MHz}, \mathrm{CDCl}_{3}\right) \delta-144.86--144.80(\mathrm{~m}, 2 \mathrm{~F}),-155.87--155.75$ 
$(\mathrm{m}, J=21.5 \mathrm{~Hz}, 1 \mathrm{~F}),-162.02--161.90(\mathrm{~m}, 2 \mathrm{~F}) . \mathbf{I R}$ (thin film, $\left.\mathrm{cm}^{-1}\right) 3019,1709,1524,1444$, $1214,1006,877,744,668$.

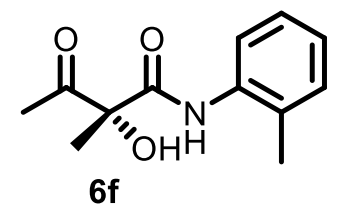

6f: Colorless oil; $8 \mathrm{~h}$, Yield: $91 \%$; Enantiomeric excess: $93 \% ;[\alpha]_{\mathrm{D}}{ }^{20}=123.65\left(\mathrm{c}=2.00, \mathrm{CHCl}_{3}\right)$. HPLC analysis: Daicel Chiralpak OJ-H, hexane/iso-propanol $=95: 5$, flow rate $=1.2 \mathrm{~mL} / \mathrm{min}, \lambda=$ $206 \mathrm{~nm}$, retention time: 14.95 (minor) and $17.06 \mathrm{~min}$ (major). ${ }^{1} \mathbf{H} \mathbf{~ N M R}\left(400 \mathrm{MHz}, \mathrm{CDCl}_{3}\right) \delta 8.89$ $-8.66(\mathrm{~m}, 1 \mathrm{H}), 8.00-7.84(\mathrm{~m}, 1 \mathrm{H}), 7.31-7.12(\mathrm{~m}, 2 \mathrm{H}), 7.07(\mathrm{~m}, 1 \mathrm{H}), 5.03(\mathrm{~s}, 1 \mathrm{H}), 2.53(\mathrm{~s}, 3 \mathrm{H})$, $2.25(\mathrm{~s}, 3 \mathrm{H}), 1.73(\mathrm{~s}, 3 \mathrm{H}) .{ }^{13} \mathrm{C}$ NMR $\left(101 \mathrm{MHz}, \mathrm{CDCl}_{3}\right) \delta 208.0,168.1,135.1,130.6,128.6,126.9$, 125.4, 121.7, 82.4, 24.8, 24.6, 17.6. ESI-HR calcd for $\mathrm{C}_{12} \mathrm{H}_{15} \mathrm{NO}_{3} \mathrm{Na}^{+}$: 244.0944, found 244.0934. IR (thin film, cm-1) 3391, 3019, 1709, 1687, 1528, 1457, 1149, 1046, 909, 877, 744, 668.<smiles>CC(=O)[C@](C)(O)C(=O)Nc1ccccc1C(C)(C)C</smiles>

6g: White solid; 8 h, Yield: 91\%; Enantiomeric excess: 99\%; $[\alpha]_{\mathrm{D}}{ }^{20}=128.63\left(\mathrm{c}=2.40, \mathrm{CHCl}_{3}\right)$. HPLC analysis: Daicel Chiralpak OJ-H, hexane/iso-propanol $=95: 5$, flow rate $=1.0 \mathrm{~mL} / \mathrm{min}, \lambda=$ $206 \mathrm{~nm}$, retention time: 7.91 (major) and $8.76 \mathrm{~min}$ (minor). ${ }^{1} \mathbf{H} \mathbf{N M R}\left(400 \mathrm{MHz}, \mathrm{CDCl}_{3}\right) \delta 9.02(\mathrm{~s}$, 1H), $7.75(\mathrm{dd}, J=7.9,1.5 \mathrm{~Hz}, 1 \mathrm{H}), 7.39(\mathrm{dd}, J=8.0,1.6 \mathrm{~Hz}, 1 \mathrm{H}), 7.31-7.20(\mathrm{~m}, 1 \mathrm{H}), 7.14(\mathrm{td}, J$ $=7.7,1.5 \mathrm{~Hz}, 1 \mathrm{H}), 5.07(\mathrm{~s}, 1 \mathrm{H}), 2.55(\mathrm{~s}, 3 \mathrm{H}), 1.75(\mathrm{~s}, 3 \mathrm{H}), 1.39(\mathrm{~s}, 9 \mathrm{H}) .{ }^{13} \mathbf{C}$ NMR $(101 \mathrm{MHz}$ $\left.\mathrm{CDCl}_{3}\right) \delta 207.7,167.8,141.6,134.6,126.9,126.7,126.0,125.7,82.5,34.5,30.5,24.6,24.5$.

ESI-HR calcd for $\mathrm{C}_{15} \mathrm{H}_{21} \mathrm{NO}_{3} \mathrm{Na}^{+}: 286.1414$, found 286.1403. IR (thin film, $\mathrm{cm}^{-1}$ ) 3019, 1709, 
$1687,1516,1446,1214,1047,877,744,668$.

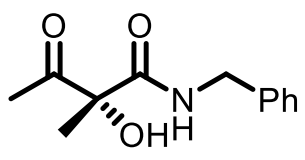

$6 h$

6h: White solid; 5 h, Yield: 90\%; Enantiomeric excess: 97\%; $[\alpha]_{\mathrm{D}}^{24}=116.51\left(\mathrm{c}=2.70, \mathrm{CHCl}_{3}\right)$.

HPLC analysis: Daicel Chiralpak OJ-H, hexane/iso-propanol $=90: 10$, flow rate $=1.0 \mathrm{~mL} / \mathrm{min}, \lambda=$ $209 \mathrm{~nm}$, retention time: 18.35 (minor) and $19.23 \mathrm{~min}$ (major). ${ }^{1} \mathbf{H} \mathbf{~ N M R}\left(400 \mathrm{MHz}, \mathrm{CDCl}_{3}\right) \delta 7.37$ $-7.19(\mathrm{~m}, 5 \mathrm{H}), 4.80(\mathrm{~s}, 1 \mathrm{H}), 4.49-4.33(\mathrm{~m}, 2 \mathrm{H}), 2.49(\mathrm{~s}, 3 \mathrm{H}), 1.65(\mathrm{~s}, 3 \mathrm{H}) .{ }^{13} \mathbf{C}$ NMR $(101 \mathrm{MHz}$, $\left.\mathrm{CDCl}_{3}\right) \delta 208.2,170.1,137.6,128.9,127.8,127.7,82.0,66.0,43.7,24.7$. ESI-HR calcd for $\mathrm{C}_{12} \mathrm{H}_{15} \mathrm{NO}_{3} \mathrm{Na}^{+}$: 244.0944, found 244.0935. IR (thin film, $\mathrm{cm}^{-1}$ ) 3416, 3019, 1709, 1675, 1517, 1361, 1214, 1150, 1046, 877, 744, 668.

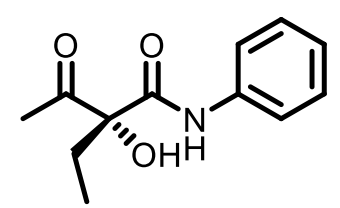

$6 \mathbf{i}$

6i: White solid; $30 \mathrm{~h}$, Yield: 38\%; Enantiomeric excess: 93\%; $[\alpha]_{\mathrm{D}}^{20}=106.5\left(\mathrm{c}=1.6, \mathrm{CHCl}_{3}\right)$.

HPLC analysis: Daicel Chiralpak AD-H, hexane/iso-propanol $=98: 2$, flow rate $=0.8 \mathrm{~mL} / \mathrm{min}, \lambda=$ $246 \mathrm{~nm}$, retention time: 8.36 (major) and $9.78 \mathrm{~min}$ (minor). ${ }^{1} \mathbf{H} \mathbf{~ N M R ~}\left(400 \mathrm{MHz}, \mathrm{CDCl}_{3}\right) \delta 8.83$ (s, 1H), $7.62-7.47(\mathrm{~m}, 2 \mathrm{H}), 7.33(\mathrm{~m}, 2 \mathrm{H}), 7.20-7.08(\mathrm{~m}, 1 \mathrm{H}), 4.91(\mathrm{~s}, 1 \mathrm{H}), 2.52(\mathrm{~s}, 3 \mathrm{H}), 2.22(\mathrm{dq}, J$ $=14.7,7.4 \mathrm{~Hz}, 1 \mathrm{H}), 2.04(\mathrm{dq}, J=14.4,7.3 \mathrm{~Hz}, 1 \mathrm{H}), 0.92(\mathrm{t}, J=7.4 \mathrm{~Hz}, 3 \mathrm{H}) .{ }^{13} \mathbf{C} \mathbf{N M R}(101 \mathrm{MHz}$, $\left.\mathrm{CDCl}_{3}\right) \delta$ 207.7, 167.6, 137.1, 129.2, 125.0, 119.9, 85.7, 30.9, 24.9, 7.8. ESI-HR calcd for $\mathrm{C}_{12} \mathrm{H}_{15} \mathrm{NO}_{3} \mathrm{Na}^{+}$: 244.0944, found 244.0937. IR (thin film, $\mathrm{cm}^{-1}$ ) 3381, 3019, 1710, 1687, 1524, 1444, 1214, 1149, 1036, 877, 744, 668. 
<smiles>CC(=O)[C@@](O)(C(=O)O)C(=O)Nc1ccccc1</smiles>

6j

6j: White solid; $30 \mathrm{~h}$, Yield: $35 \%$; Enantiomeric excess: $96 \%$; $[\alpha]_{\mathrm{D}}{ }^{20}=72.19\left(\mathrm{c}=1.72, \mathrm{CHCl}_{3}\right)$.

HPLC analysis: Daicel Chiralpak AD-H, hexane/iso-propanol $=98: 2$, flow rate $=0.8 \mathrm{~mL} / \mathrm{min}, \lambda=$ $245 \mathrm{~nm}$, retention time: 5.86 (major) and $6.22 \mathrm{~min}$ (minor). ${ }^{1} \mathbf{H} \mathbf{N M R}\left(400 \mathrm{MHz}, \mathrm{CDCl}_{3}\right) \delta 8.83(\mathrm{~s}$, 1H), $7.59-7.48(\mathrm{~m}, 2 \mathrm{H}), 7.37-7.30(\mathrm{~m}, 2 \mathrm{H}), 7.19-7.08(\mathrm{~m}, 1 \mathrm{H}), 4.94(\mathrm{~s}, 1 \mathrm{H}), 2.51(\mathrm{~s}, 3 \mathrm{H})$, $2.18(\mathrm{ddd}, J=13.1,11.2,3.8 \mathrm{~Hz}, 1 \mathrm{H}), 1.96(\mathrm{ddd}, J=13.2,11.1,3.6 \mathrm{~Hz}, 1 \mathrm{H}), 1.24-1.28(\mathrm{~m}, 16 \mathrm{H})$, $0.87(\mathrm{t}, J=6.8 \mathrm{~Hz}, 3 \mathrm{H}) .{ }^{13} \mathbf{C}$ NMR $\left(101 \mathrm{MHz}, \mathrm{CDCl}_{3}\right) \delta 207.7,167.7,137.1,129.2,125.0,119.9$, 85.5, 37.7, 32.0, 29.7, 29.6, 29.6, 29.5, 29.4, 24.9, 23.5, 22.8, 14.2. ESI-HR calcd for $\mathrm{C}_{20} \mathrm{H}_{31} \mathrm{NO}_{3} \mathrm{Na}^{+}$: 356.2196, found 356.2186. IR (thin film, $\mathrm{cm}^{-1}$ ) 3019, 1710, 1687, 1525, 1046, $744,668$.

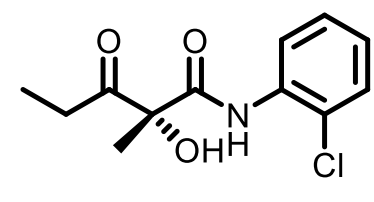

6k

6k: Colorless oil; 30 h, Yield: 38\%; Enantiomeric excess: $96 \%$; $[\alpha]_{\mathrm{D}}{ }^{24}=58.01\left(\mathrm{c}=1.94, \mathrm{CHCl}_{3}\right)$. HPLC analysis: Daicel Chiralpak AD-H, hexane/iso-propanol $=98: 2$, flow rate $=0.8 \mathrm{~mL} / \mathrm{min}, \lambda=$ $245 \mathrm{~nm}$, retention time: 5.86 (major) and $6.22 \mathrm{~min}$ (minor). ${ }^{1} \mathbf{H} \mathbf{~ N M R}\left(400 \mathrm{MHz}, \mathrm{CDCl}_{3}\right) \delta 9.49(\mathrm{~s}$, $1 \mathrm{H}), 8.35(\mathrm{dd}, J=8.3,1.6 \mathrm{~Hz}, 1 \mathrm{H}), 7.38(\mathrm{dd}, J=8.0,1.5 \mathrm{~Hz}, 1 \mathrm{H}), 7.33-7.22(\mathrm{~m}, 1 \mathrm{H}), 7.07(\mathrm{td}, J$ = 7.7, $1.6 \mathrm{~Hz}, 1 \mathrm{H}), 5.09(\mathrm{~s}, 1 \mathrm{H}), 3.12(\mathrm{dq}, J=19.2,7.2 \mathrm{~Hz}, 1 \mathrm{H}), 2.81(\mathrm{dq}, J=19.2,7.2 \mathrm{~Hz}, 1 \mathrm{H})$, $1.73(\mathrm{~s}, 3 \mathrm{H}), 1.12(\mathrm{t}, J=7.2 \mathrm{~Hz}, 3 \mathrm{H}) .{ }^{13} \mathbf{C}$ NMR $\left(101 \mathrm{MHz}, \mathrm{CDCl}_{3}\right) \delta 210.1,168.7,134.0,129.4$, 127.8, 125.3, 123.8, 121.1, 82.3, 30.1, 24.9, 7.8. ESI-HR calcd for $\mathrm{C}_{12} \mathrm{H}_{14} \mathrm{ClNO}_{3} \mathrm{Na}^{+}$: 278.0554, 
found 278.0547. IR (thin film, $\mathrm{cm}^{-1}$ ) 3019, 1710, 1692, 1525, 1214, 1036, 744, 668 .<smiles>O=C1CCCC[C@]1(O)C(=O)Nc1ccccc1</smiles>

61

6l: White solid; Yield: $55 \%$. Enantiomeric excess: $90 \% ;[\alpha]_{\mathrm{D}}{ }^{20}=138.78\left(\mathrm{c}=1.94, \mathrm{CHCl}_{3}\right)$. HPLC analysis: Daicel Chiralpak OJ-H, hexane/iso-propanol $=90: 10$, flow rate $=1.0 \mathrm{~mL} / \mathrm{min}, \lambda=244$ nm, retention time: 22.01 (minor) and 28.99 min (major). ${ }^{1} \mathbf{H} \mathbf{N M R}\left(400 \mathrm{MHz}, \mathrm{CDCl}_{3}\right) \delta 8.84(\mathrm{~s}$, $1 \mathrm{H}), 7.52(\mathrm{~d}, J=7.7 \mathrm{~Hz}, 2 \mathrm{H}), 7.38-7.29(\mathrm{~m}, 2 \mathrm{H}), 7.13(\mathrm{t}, J=7.4 \mathrm{~Hz}, 1 \mathrm{H}), 5.00(\mathrm{~s}, 1 \mathrm{H}), 3.34(\mathrm{dt}$, $J=13.6,6.4 \mathrm{~Hz}, 1 \mathrm{H}), 2.75-2.61(\mathrm{~m}, 1 \mathrm{H}), 2.59-2.50(\mathrm{~m}, 1 \mathrm{H}), 2.45(\mathrm{dt}, J=13.2,3.7 \mathrm{~Hz}, 1 \mathrm{H})$, $2.25(\mathrm{~m}, 1 \mathrm{H}), 1.83-1.66(\mathrm{~m}, 3 \mathrm{H}) .{ }^{13} \mathbf{C}$ NMR $\left(101 \mathrm{MHz}, \mathrm{CDCl}_{3}\right) \delta$ 209.3, 168.1, 137.1, 129.2, 124.9, 120.0, 81.5, 41.7, 39.2, 27.9, 22.3. ESI-HR calcd for $\mathrm{C}_{14} \mathrm{H}_{15} \mathrm{NO}_{3} \mathrm{Na}^{+}: 256.0944$, found 256.0935. IR (thin film, $\mathrm{cm}^{-1}$ ) 3386, 3019, 1710, 1686, 1524, 1442, 1214, 1046, 744, 668.<smiles>Cc1ccc(NC(=O)[C@]2(O)CCCCC2=O)cc1</smiles>

$6 \mathrm{~m}$

6m: White solid; Yield: $80 \%$; Enantiomeric excess: 95\% ee; $[\alpha]_{\mathrm{D}}^{20}=93.33\left(\mathrm{c}=0.80, \mathrm{CHCl}_{3}\right)$. HPLC analysis: Daicel Chiralpak OJ-H, hexane/iso-propanol $=90: 10$, flow rate $=1.0 \mathrm{~mL} / \mathrm{min}, \lambda=$ $207 \mathrm{~nm}$, retention time: 12.58 (minor) and $13.89 \mathrm{~min}$ (major). ${ }^{1} \mathbf{H} \mathbf{~ N M R}\left(400 \mathrm{MHz}, \mathrm{CDCl}_{3}\right) \delta 8.76$ (s, 1H), $7.40(\mathrm{~d}, 8.4 \mathrm{~Hz}, 2 \mathrm{H}), 7.13(\mathrm{~d}, J=8.1 \mathrm{~Hz}, 2 \mathrm{H}), 4.96(\mathrm{~s}, 1 \mathrm{H}), 3.34(\mathrm{dt}, J=13.6,6.4 \mathrm{~Hz}, 1 \mathrm{H})$, $2.65(\mathrm{ddt}, J=13.4,4.2,2.1 \mathrm{~Hz}, 1 \mathrm{H}), 2.58-2.39(\mathrm{~m}, 2 \mathrm{H}), 2.31(\mathrm{~s}, 3 \mathrm{H}), 2.25$ (ddt, $J=13.1,4.0,2.0$ $\mathrm{Hz}, 1 \mathrm{H}), 1.81-1.66(\mathrm{~m}, 3 \mathrm{H}) .{ }^{13} \mathbf{C}$ NMR $\left(101 \mathrm{MHz}, \mathrm{CDCl}_{3}\right) \delta 209.4,167.9,134.5,129.7,120.0$, 81.5, 41.8, 39.3, 27.9, 22.3, 21.0. ESI-HR calcd for $\mathrm{C}_{14} \mathrm{H}_{17} \mathrm{NO}_{3} \mathrm{Na}^{+}$: 270.1101, found 270.1090. 
IR (thin film, $\mathrm{cm}^{-1}$ ) 3019, 1709, 1681, 1523, 1422, 1214, 1046, 744, 668.

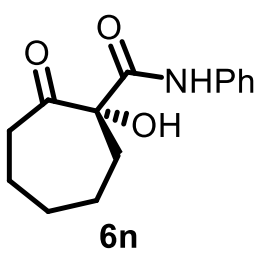

6n: White solid; Yield: 87\%; Enantiomeric excess: 98\%; $[\alpha]_{\mathrm{D}}{ }^{20}=-5.25\left(\mathrm{c}=0.90, \mathrm{CHCl}_{3}\right) . \mathbf{H P L C}$ analysis: Daicel Chiralpak OJ-H, hexane/iso-propanol $=95: 5$, flow rate $=1.0 \mathrm{~mL} / \mathrm{min}, \lambda=245 \mathrm{~nm}$, retention time: 31.18 (minor) and 34.21 min (major). ${ }^{1} \mathbf{H} \mathbf{~ N M R}\left(400 \mathrm{MHz}, \mathrm{CDCl}_{3}\right) \delta 8.86(\mathrm{~s}, 1 \mathrm{H})$, $7.60-7.46(\mathrm{~m}, 2 \mathrm{H}), 7.38-7.27(\mathrm{~m}, 2 \mathrm{H}), 7.18-7.02(\mathrm{~m}, 1 \mathrm{H}), 4.93(\mathrm{~s}, 1 \mathrm{H}), 3.47$ (ddd, $J=13.5$, 11.0, $3.0 \mathrm{~Hz}, 1 \mathrm{H}), 2.67-2.57(\mathrm{~m}, 1 \mathrm{H}), 2.56-2.44(\mathrm{~m}, 1 \mathrm{H}), 2.17-2.04(\mathrm{~m}, 2 \mathrm{H}), 1.98-1.81(\mathrm{~m}$, 2H), $1.60-1.33(\mathrm{~m}, 2 \mathrm{H}), 1.24-1.09(\mathrm{~m}, 1 \mathrm{H}) .{ }^{13} \mathrm{C} \mathbf{N M R}\left(101 \mathrm{MHz}, \mathrm{CDCl}_{3}\right) \delta 211.2,168.3,137.0$, 129.2, 124.9, 119.8, 85.2, 77.5, 77.2, 76.8, 39.8, 34.0, 30.5, 28.3, 23.9. ESI-HR calcd for $\mathrm{C}_{14} \mathrm{H}_{17} \mathrm{NO}_{3} \mathrm{Na}^{+}$: 270.1101, found 270.1090. IR (thin film, $\mathrm{cm}^{-1}$ ) 3019, 1709, 1681, 1526, 1214 , $1045,744,668$.

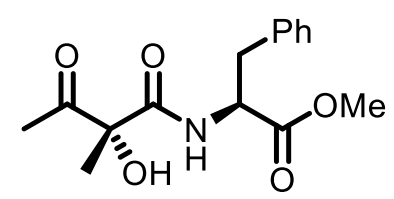

60

6o: White solid; 6 h, Yield: $60 \%$; Diastereomeric ratio $>20: 1 ;[\alpha]_{\mathrm{D}}{ }^{24}=90.80\left(\mathrm{c}=3.7, \mathrm{CHCl}_{3}\right) .{ }^{1} \mathbf{H}$ $\operatorname{NMR}\left(400 \mathrm{MHz}, \mathrm{CDCl}_{3}\right) \delta 7.33-7.23(\mathrm{~m}, 4 \mathrm{H}), 7.14-7.12(\mathrm{~m}, 2 \mathrm{H}), 4.77(\mathrm{ddd}, J=8.4,7.1,5.6$ $\mathrm{Hz}, 1 \mathrm{H}), 4.71(\mathrm{~s}, 1 \mathrm{H}), 3.71(\mathrm{~s}, 3 \mathrm{H}), 3.18(\mathrm{dd}, J=13.8,5.5 \mathrm{~Hz}, 1 \mathrm{H}), 3.04(\mathrm{dd}, J=13.8,7.1 \mathrm{~Hz}, 1 \mathrm{H})$, $2.40(\mathrm{~s}, 3 \mathrm{H}), 1.52(\mathrm{~s}, 3 \mathrm{H}) .{ }^{13} \mathrm{C}$ NMR $\left(101 \mathrm{MHz}, \mathrm{CDCl}_{3}\right) \delta 207.4,171.4,169.9,135.7,129.3,128.8$, 127.4, 81.8, 53.4, 52.5, 38.1, 24.6, 24.5. ESI-HR calcd for $\mathrm{C}_{15} \mathrm{H}_{19} \mathrm{NO}_{5} \mathrm{Na}^{+}$: 316.1155 , found 316.1146. IR (thin film, $\mathrm{cm}^{-1}$ ) 3019, 1744, 1679, 1513, 1423, 1214, 744, 668. 


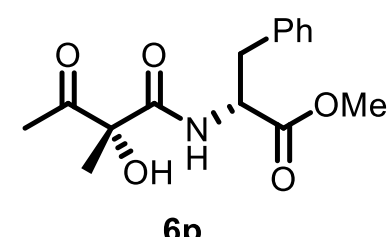

6p: White solid; 6 h, Yield: 60\%; Diastereomeric ratio: $>20: 1 ;[\alpha]_{D^{24}}=69.54\left(\mathrm{c}=1.8, \mathrm{CHCl}_{3}\right)$. ${ }^{1} \mathbf{H}$ NMR $\left(400 \mathrm{MHz}, \mathrm{CDCl}_{3}\right) \delta 7.38(\mathrm{~d}, J=8.5 \mathrm{~Hz}, 1 \mathrm{H}), 7.34-7.20(\mathrm{~m}, 3 \mathrm{H}), 7.07-6.98(\mathrm{~m}, 2 \mathrm{H})$, $4.79(\mathrm{td}, J=8.1,5.3 \mathrm{~Hz}, 1 \mathrm{H}), 4.69(\mathrm{~s}, 1 \mathrm{H}), 3.75(\mathrm{~s}, 3 \mathrm{H}), 3.15(\mathrm{dd}, J=13.8,5.3 \mathrm{~Hz}, 1 \mathrm{H}), 2.94(\mathrm{dd}$, $J=13.8,7.7 \mathrm{~Hz}, 1 \mathrm{H}), 2.27(\mathrm{~s}, 3 \mathrm{H}), 1.55(\mathrm{~s}, 3 \mathrm{H}) .{ }^{13} \mathbf{C} \mathbf{N M R}\left(101 \mathrm{MHz}, \mathrm{CDCl}_{3}\right) \delta 207.5,171.5$, $169.8,135.8,129.3,128.7,127.3,81.9,53.3,52.6,38.4,24.3,24.2$. ESI-HR calcd for $\mathrm{C}_{15} \mathrm{H}_{19} \mathrm{NO}_{5} \mathrm{Na}^{+}$: 316.1155, found 316.1146. IR (thin film, $\mathrm{cm}^{-1}$ ) 3019, 1744, 1679, 1513, 1423 , $1214,744,668$.

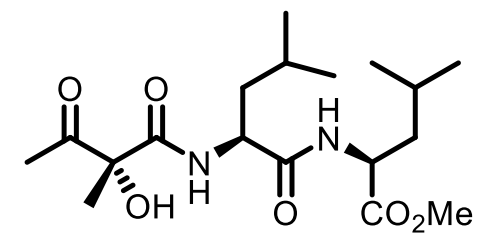

6q

6q: Colorless solid; $9 \mathrm{~h}$, Yield: 50\%; Diastereomeric ratio: $>20: 1 ;[\alpha]_{\mathrm{D}}^{24}=32.73(\mathrm{c}=2.0$, $\left.\mathrm{CHCl}_{3}\right) .{ }^{1} \mathbf{H} \mathbf{N M R}\left(400 \mathrm{MHz}, \mathrm{CDCl}_{3}\right) \delta 7.29(\mathrm{~s}, 1 \mathrm{H}), 6.30(\mathrm{~d}, J=8.3 \mathrm{~Hz}, 1 \mathrm{H}), 4.79(\mathrm{~s}, 1 \mathrm{H}), 4.57$ $(\mathrm{td}, J=8.6,5.0 \mathrm{~Hz}, 1 \mathrm{H}), 4.34(\mathrm{td}, J=8.4,5.8 \mathrm{~Hz}, 1 \mathrm{H}), 3.73(\mathrm{~s}, 3 \mathrm{H}), 2.45(\mathrm{~s}, 3 \mathrm{H}), 1.76-1.47(\mathrm{~m}$, 6H), $1.63(\mathrm{~s}, 3 \mathrm{H}), 0.98-0.86(\mathrm{~m}, 12 \mathrm{H}) .{ }^{13} \mathbf{C}$ NMR $\left(101 \mathrm{MHz}, \mathrm{CDCl}_{3}\right) \delta$ 207.3, 173.2, 171.0, 170.6, 81.9, 52.5, 52.1, 50.8, 41.6, 40.6, 24.9, 24.9, 24.6, 24.5, 23.0, 22.9, 22.2, 21.9. ESI-HR calcd for $\mathrm{C}_{15} \mathrm{H}_{19} \mathrm{NO}_{5} \mathrm{Na}^{+}$: 395.2153, found 395.2141. IR (thin film, $\mathrm{cm}^{-1}$ ) 3019, 1740, 1672, 1516, 1424 , 1214, 744, 668 . 


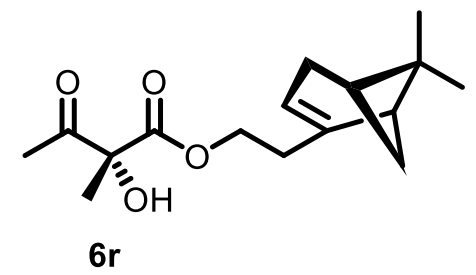

6r: Colorless oil; 6 h, Yield: 79\%; Diastereomeric ratio: 14:1; $[\alpha]_{\mathrm{D}}^{20}=-10.16\left(\mathrm{c}=2.11, \mathrm{CHCl}_{3}\right)$. ${ }^{1} \mathbf{H}$ NMR $\left(400 \mathrm{MHz}, \mathrm{CDCl}_{3}\right) \delta 5.27(\mathrm{~m} \mathrm{1H}), 4.27-4.11(\mathrm{~m}, 3 \mathrm{H}), 2.38-2.27(\mathrm{~m}, 3 \mathrm{H}), 2.25(\mathrm{~s}$, $3 \mathrm{H}), 2.23-2.13(\mathrm{~m}, 2 \mathrm{H}), 2.10-1.97(\mathrm{~m}, 2 \mathrm{H}), 1.56(\mathrm{~s}, 3 \mathrm{H}), 1.26(\mathrm{~s}, 3 \mathrm{H}), 1.10(\mathrm{~d}, J=8.5 \mathrm{~Hz}, 1 \mathrm{H})$, $0.80(\mathrm{~s}, 3 \mathrm{H}) .{ }^{13} \mathrm{C}$ NMR $\left(101 \mathrm{MHz}, \mathrm{CDCl}_{3}\right) \delta 204.8,171.4,143.5,119.4,81.1,77.5,77.2,76.8$ 64.7, 45.7, 40.8, 38.1, 35.9, 31.7, 31.5, 26.3, 24.4, 21.9, 21.2. ESI-HR calcd for $\mathrm{C}_{16} \mathrm{H}_{24} \mathrm{O}_{4} \mathrm{Na}^{+}$: 303.1567, found 303.1559. IR (thin film, $\mathrm{cm}^{-1}$ ) 3019, 1721, 1214, 1046, 744, 668.

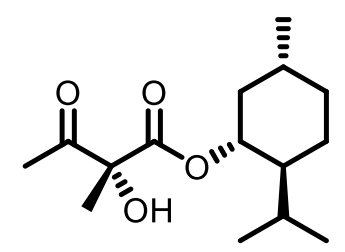

6s

6s: Colorless oil; $6 \mathrm{~h}$, Yield: 87\%; Diastereomeric ratio: $>20: 1 ;[\alpha]_{\mathrm{D}}^{24}=-23.19\left(\mathrm{c}=1.90, \mathrm{CHCl}_{3}\right)$. ${ }^{1} \mathbf{H}$ NMR $\left(400 \mathrm{MHz}, \mathrm{CDCl}_{3}\right) \delta 4.73(\mathrm{td}, J=10.9,4.4 \mathrm{~Hz}, 1 \mathrm{H}), 4.12(\mathrm{~s}, 1 \mathrm{H}), 2.23(\mathrm{~s}, 3 \mathrm{H})$, 1.98-1.92 (m, 1H), $1.81-1.76(\mathrm{~m}, 1 \mathrm{H}), 1.71-1.62(\mathrm{~m}, 2 \mathrm{H}), 1.57-1.38(\mathrm{~m}, 2 \mathrm{H}), 1.55(\mathrm{~s}, 3 \mathrm{H})$, $1.09-0.95(\mathrm{~m}, 2 \mathrm{H}), 0.92-0.81(\mathrm{~m}, 1 \mathrm{H}), 0.90(\mathrm{~d}, 6.8 \mathrm{~Hz}, 3 \mathrm{H}), 0.87(\mathrm{~d}, 6.8 \mathrm{~Hz}, 3 \mathrm{H}), 0.73(\mathrm{~d}, J=$ 7.0 Hz, 3H). ${ }^{13} \mathbf{C}$ NMR $\left(101 \mathrm{MHz}, \mathrm{CDCl}_{3}\right) \delta 204.8,171.3,81.1,77.5,77.2,76.9,46.9,40.5,34.2$, 31.5, 26.1, 24.3, 23.3, 22.1, 21.7, 20.8, 16.1. ESI-HR calcd for $\mathrm{C}_{15} \mathrm{H}_{26} \mathrm{O}_{4} \mathrm{Na}^{+}:$293.1723, found 293.1714. IR (thin film, $\mathrm{cm}^{-1}$ ) 3497, 3020, 2960, 2873, 1717,1215, 744, 668. 


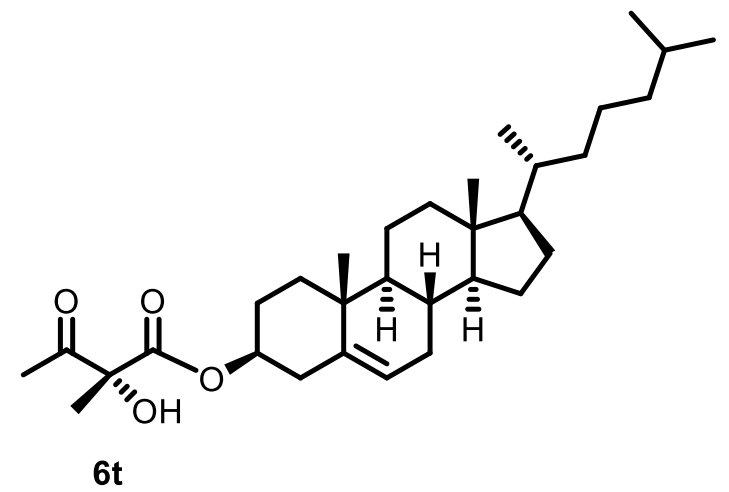

6t: White solid; 6 h, Yield: $40 \%$; Diastereomeric ratio: $>20: 1$ dr. $[\alpha]_{\mathrm{D}^{20}}=-5.05\left(\mathrm{c}=3.50, \mathrm{CHCl}_{3}\right)$.

${ }^{1} \mathbf{H}$ NMR $\left(400 \mathrm{MHz}, \mathrm{CDCl}_{3}\right) \delta 5.38(\mathrm{~d}, J=4.8 \mathrm{~Hz}, 1 \mathrm{H}), 4.69(\mathrm{~m}, 1 \mathrm{H}), 4.12(\mathrm{~s}, 1 \mathrm{H}), 2.28-2.40(\mathrm{~m}$, 2H), $2.26(\mathrm{~s}, 3 \mathrm{H}), 2.06-1.77(\mathrm{~m}, 5 \mathrm{H}), 1.66-0.95(\mathrm{~m}, 21 \mathrm{H}), 1.56(\mathrm{~s}, 3 \mathrm{H}), 1.01(\mathrm{~s}, 3 \mathrm{H}), 0.91(\mathrm{~d}, J$ $=6.5 \mathrm{~Hz}, 3 \mathrm{H}), 0.86(\mathrm{~d}, J=6.6,3 \mathrm{H}), 0.85(\mathrm{~d}, J=6.6,3 \mathrm{H}), 0.67(\mathrm{~s}, 3 \mathrm{H}) .{ }^{13} \mathbf{C} \mathbf{N M R}(101 \mathrm{MHz}$, $\left.\mathrm{CDCl}_{3}\right) \delta 204.9,171.0,139.1,123.4,81.1,76.7,56.8,56.3,50.2,42.5,39.9,39.7,37.8,37.0,36.7$, $36.3,35.9,32.1,32.0,28.4,28.2,27.6,24.4,24.3,24.0,23.0,22.7,21.8,21.2,19.4,18.9,12.0$. ESI-HR calcd for $\mathrm{C}_{32} \mathrm{H}_{52} \mathrm{O}_{4} \mathrm{Na}^{+}:$523.3758, found 523.3746. IR (thin film, $\mathrm{cm}^{-1}$ ) 3019, 2950, 1719, $1214,744,668$.

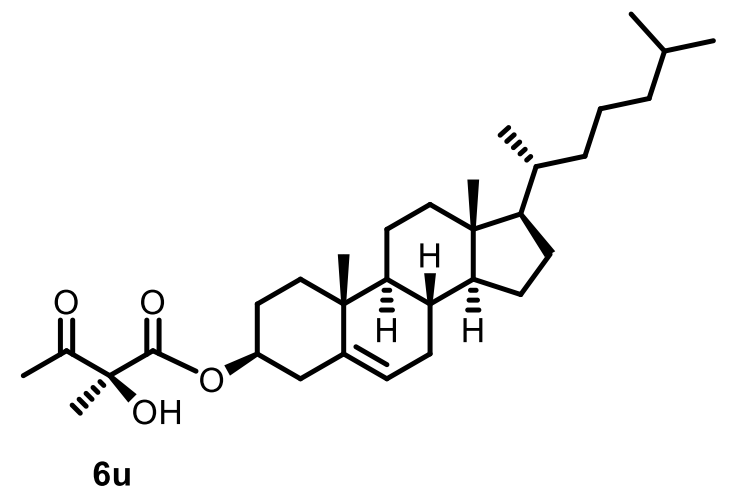

6u: White solid; $6 \mathrm{~h}$, Yield: $40 \%$; Diastereomeric ratio: $>20: 1 \mathrm{dr} .[\alpha]_{\mathrm{D}}^{20}=-34.08(\mathrm{c}=3.55$, $\left.\mathrm{CHCl}_{3}\right) .{ }^{1} \mathbf{H} \mathbf{N M R}\left(400 \mathrm{MHz}, \mathrm{CDCl}_{3}\right) \delta 5.38(\mathrm{~d}, J=4.8 \mathrm{~Hz}, 1 \mathrm{H}), 4.69(\mathrm{~m}, 1 \mathrm{H}), 4.12(\mathrm{~s}, 1 \mathrm{H}), 2.28$ $-2.40(\mathrm{~m}, 2 \mathrm{H}), 2.26(\mathrm{~s}, 3 \mathrm{H}), 2.06-1.77(\mathrm{~m}, 5 \mathrm{H}), 1.66-0.95(\mathrm{~m}, 21 \mathrm{H}), 1.56(\mathrm{~s}, 3 \mathrm{H}), 1.01(\mathrm{~s}, 3 \mathrm{H})$, 
$0.91(\mathrm{~d}, J=6.5 \mathrm{~Hz}, 3 \mathrm{H}), 0.87(\mathrm{~d}, J=6.6,3 \mathrm{H}), 0.86(\mathrm{~d}, J=6.6,3 \mathrm{H}), 0.67(\mathrm{~s}, 3 \mathrm{H}) .{ }^{13} \mathbf{C}$ NMR $(101$

$\left.\mathrm{MHz}, \mathrm{CDCl}_{3}\right) \delta 204.9,171.0,139.1,123.4,81.1,76.7,56.8,56.3,50.2,42.5,39.9,39.7,37.8,37.0$,

$36.7,36.3,35.9,32.1,32.0,28.4,28.2,27.6,24.4,24.3,24.0,23.0,22.7,21.8,21.2,19.4,18.9$,

12.0. ESI-HR calcd for $\mathrm{C}_{32} \mathrm{H}_{52} \mathrm{O}_{4} \mathrm{Na}^{+}:$523.3758, found 523.3745. IR (thin film, $\mathrm{cm}^{-1}$ ) 3019, 2950,

$1719,1214,744,668$.

General procedure for the Gram-scale experiment: An oven-dried tube equipped with stir bar was charged with 1a $(10 \mathrm{mmol})$ and aminocatalyst $3 \mathbf{a} / \mathrm{NHTf}_{2}(10 \mathrm{~mol} \%)$. After dissolution in mixed solvent of toluene and 1,2-dichloroethane $(25 \mathrm{~mL})$, trifluoroacetophenone $4 \mathbf{a}(10 \mathrm{~mol} \%)$ was added to the vial. Then $\mathrm{H}_{2} \mathrm{O}_{2}$ (30 wt.\% in water, $15 \mathrm{mmol}$ ) was added via syringe. Upon completion of the addition, the reaction was conducted at room temperature for 8 hours (TLC analysis). The solvent was removed under reduced pressure and the residue was purified by silica gel chromatography with the utilization of ethyl acetate/hexane (1:8) to give product $2 \mathbf{a}$ (1.61g, $73 \%$ yield, $95 \%$ ee) as a colorless oil. The enantiomeric excess was determined by HPLC $\left(\mathrm{AS}-\mathrm{H}^{*}\right)$.

\section{Determination of Absolute Configuration}

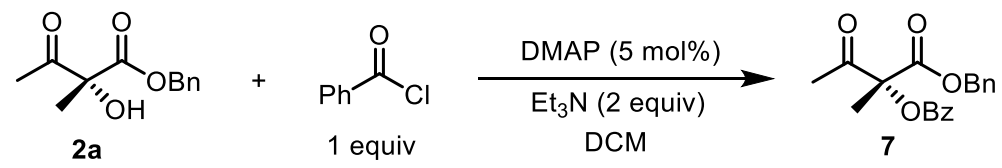

The solution of 2a (0.05 M in DCM) was treated with DMAP (5 mol\%), benzoyl chloride (1 equiv) and $\mathrm{NEt}_{3}$ ( 2 equiv), then allowed to stir for $3 \mathrm{~h}$. At this time, the reaction mixture was concentrated under reduced pressure and purified by silica gel chromatography with ethyl acetate/hexane (1:8) to afford 7 (90\% yield, $96 \%$ ee) as a colorless oil. HPLC analysis: Daicel Chiralpak OJ-H, 
hexane/iso-propanol $=95: 5$, flow rate $=1.0 \mathrm{~mL} / \mathrm{min}, \lambda=230 \mathrm{~nm}$, retention time: 34.02 (major) and $38.45 \min$ (minor). The absolute configuration of product 2 was determined according to the optical rotation of $7\left([\alpha]_{\mathrm{D}}^{26}=-13.5\left(\mathrm{c}=2.28, \mathrm{CHCl}_{3}\right)\right)$ compared with the $\alpha$-benzoyloxylation product reported by our group ${ }^{13}$.

\section{Synthesis of Biologically Active Compounds}

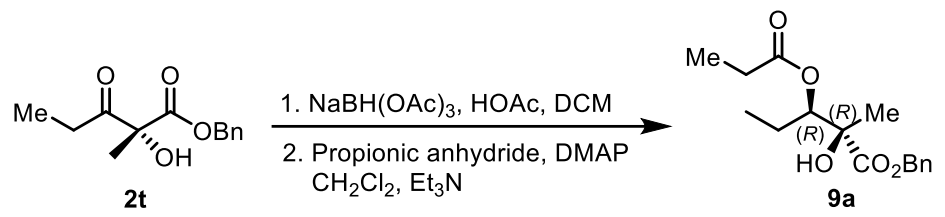

Reduction with $\mathrm{NaBH}(\mathrm{OAc})_{3}$ : To a solution of enantiomerically pure $2 \mathbf{t}$ (47 mg, $0.2 \mathrm{mmol}, 1.0$ equiv) in $\mathrm{CH}_{2} \mathrm{Cl}_{2}(2 \mathrm{~mL})$ was added $\mathrm{HOAc}(0.01 \mathrm{mmol}, 5 \mathrm{~mol} \%)$ followed by addition of $\mathrm{NaBH}(\mathrm{OAc})_{3}\left(42 \mathrm{mg}, 0.2 \mathrm{mmol}, 1.0\right.$ equiv) at $0{ }^{\circ} \mathrm{C}$. Upon completion of the reaction (approx. 30 min) the reaction was quenched with saturated aqueous $\mathrm{NH}_{4} \mathrm{Cl}(5 \mathrm{~mL})$ then extracted with $5 \mathrm{~mL}$ of $\mathrm{CH}_{2} \mathrm{Cl}_{2} 3$ times. The combined organic layers were dried over anhydrous $\mathrm{Na}_{2} \mathrm{SO}_{4}$, concentrated; and the residue was purified by column chromatography to obtain $\mathbf{8 a}$ (42.46 mg, $95 \%$ yield, $96 \%$ ee). Analysis of HPLC demonstrated a 7:1 diastereoselectivity. $[\alpha]_{\mathrm{D}}^{26}=12.24\left(\mathrm{c}=1.5, \mathrm{CHCl}_{3}\right)$; Major diastereoisomer: ${ }^{1} \mathbf{H}$ NMR $\left(400 \mathrm{MHz}, \mathrm{CDCl}_{3}\right) \delta 7.43-7.29(\mathrm{~m}, 5 \mathrm{H}), 5.22(\mathrm{~s}, 2 \mathrm{H}), 3.53-$ $3.45(\mathrm{~m}, 1 \mathrm{H}), 3.37(\mathrm{~s}, 1 \mathrm{H}), 2.01(\mathrm{~d}, \mathrm{~J}=8.4 \mathrm{~Hz}, 1 \mathrm{H}), 1.46(\mathrm{~s}, 3 \mathrm{H}), 1.42-1.29(\mathrm{~m}, 2 \mathrm{H}), 0.96(\mathrm{t}, \mathrm{J}=$ $7.4 \mathrm{~Hz}, 3 \mathrm{H}) ;{ }^{13} \mathbf{C} \mathbf{~ N M R}\left(101 \mathrm{MHz}, \mathrm{CDCl}_{3}\right) \delta 175.5,135.1,128.82,128.77,128.4,77.9,77.3,67.8$, 25.0, 22.6, 11.0. HPLC analysis: Daicel Chiralpak AD-H, hexane/iso-propanol = 95:5, flow rate = $0.8 \mathrm{~mL} / \mathrm{min}, \lambda=209 \mathrm{~nm}$, retention time: 20.67 (minor) and $23.49 \mathrm{~min}$ (major). ESI-HR calcd for $\mathrm{C}_{13} \mathrm{H}_{18} \mathrm{O}_{4} \mathrm{Na}^{+}:$261.1097, found 261.1080. IR (thin film, $\mathrm{cm}^{-1}$ ): 3019, 1730, 1214, 744, 668;

Chemoselective acylation with propionic anhydride: To a solution of $8 \mathbf{a}$ in $\mathrm{CH}_{2} \mathrm{Cl}_{2}(2.0 \mathrm{~mL})$ was 
added DMAP (2.3 mg, $0.02 \mathrm{mmol}, 0.1$ equiv), $\mathrm{Et}_{3} \mathrm{~N}$ ( $26 \mathrm{uL}, 0.2 \mathrm{mmol}, 1$ equiv), and propionic anhydride ( $25 \mathrm{uL}, 0.2 \mathrm{mmol}, 1$ equiv). The reaction was stirred for 2 hours at room temperature, quenched with saturated aqueous $\mathrm{NaHCO}_{3}$, and stirred for an additional 30 minutes. The reaction mixture was extracted with $\mathrm{CH}_{2} \mathrm{Cl}_{2}(4 \times 2 \mathrm{~mL})$. The organic layers were combined, dried over $\mathrm{Na}_{2} \mathrm{SO}_{4}$, and concentrated. The residue was purified by column chromatography to obtain $9 \mathbf{a}$ $(85 \%$ yield, $96 \%$ ee $)$ as a colorless oil. $[\alpha]_{\mathrm{D}}{ }^{26}=-1.27\left(\mathrm{c}=1.4, \mathrm{CHCl}_{3}\right) ;{ }^{1} \mathbf{H}$ NMR $(400 \mathrm{MHz}$, $\left.\mathrm{CDCl}_{3}\right) \delta 7.37-7.21(\mathrm{~m}, 5 \mathrm{H}), 5.13(\mathrm{ABq}, J=12 \mathrm{~Hz}, 2 \mathrm{H}), 5.00(\mathrm{dd}, J=10.5,2.9 \mathrm{~Hz}, 1 \mathrm{H}), 3.24(\mathrm{~s}$, 1H), $2.33-2.19(\mathrm{~m}, 2 \mathrm{H}), 1.61-1.51(\mathrm{~m}, 1 \mathrm{H}), 1.38-1.25(\mathrm{~m}, 1 \mathrm{H}), 1.29(\mathrm{~s}, 1 \mathrm{H}), 1.05(\mathrm{t}, J=7.6$ $\mathrm{Hz}, 3 \mathrm{H}), 0.73(\mathrm{t}, J=7.4 \mathrm{~Hz}, 3 \mathrm{H}) ;{ }^{13} \mathbf{C}$ NMR $\left(101 \mathrm{MHz}, \mathrm{CDCl}_{3}\right) \delta 175.1,174.5,135.0,128.81$, $128.81,128.5,77.8,76.3,68.2,27.7,22.6,22.4,10.4,9.4$. HPLC analysis (>20:1 $\mathrm{dr}$ for racemic compound): Daicel Chiralpak OJ-H, hexane/iso-propanol $=95: 5$, flow rate $=1.0 \mathrm{~mL} / \mathrm{min}, \lambda=230$ $\mathrm{nm}$, retention time: 10.55 (major) and $12.58 \mathrm{~min}$ (minor). ESI-HR calcd for $\mathrm{C}_{16} \mathrm{H}_{22} \mathrm{O}_{5} \mathrm{Na}^{+}$: 317.1359, found 317.1347. IR (thin film, $\mathrm{cm}^{-1}$ ): 3019, 1733, 1214, 929, 909, 744, 668.

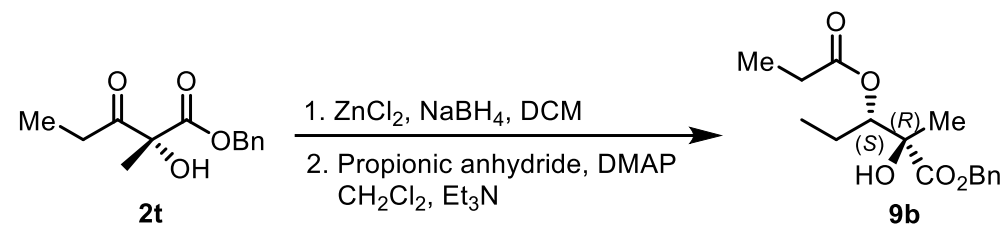

Reduction with $\mathrm{NaBH}_{4}$ : A solution of $\mathrm{NaBH}_{4}(7.57 \mathrm{mg}, 0.20 \mathrm{mmol}, 1$ equiv) in THF (1.0 ml) under nitrogen atmosphere, at $-40{ }^{\circ} \mathrm{C}$ was added $\mathrm{ZnCl}_{2}(1.0$ equiv, $0.20 \mathrm{mmol}, 0.20 \mathrm{ml}$ of $1.0 \mathrm{M}$ solution). Then a solution of enantiomerically pure $2 \mathbf{t}(47 \mathrm{mg}, 0.20 \mathrm{mmol}, 1.0$ equiv) in $0.5 \mathrm{ml}$ of THF was added to the reaction mixture, and this solution was stirred for $30 \mathrm{~min}$. The reaction was quenched with saturated aqueous $\mathrm{NH}_{4} \mathrm{Cl}(5 \mathrm{~mL})$ then extracted with $10 \mathrm{~mL}$ of diethyl ether 3 times. The combined organic layers were dried over anhydrous $\mathrm{Na}_{2} \mathrm{SO}_{4}$, concentrated; and the residue was purified by column chromatography to obtain $\mathbf{8 b}(44.35 \mathrm{mg}, 99 \%$ yield, $96 \% e e$ ) as 
colorless solid. $[\alpha]_{\mathrm{D}}^{26}=0.542\left(\mathrm{c}=1.6, \mathrm{CHCl}_{3}\right)$; Analysis of HPLC demonstrated a 16:1 diastereoselectivity. Major diastereoisomer: ${ }^{1} \mathbf{H}$ NMR (400 MHz, $\left.\mathrm{CDCl}_{3}\right) \delta 7.45-7.29(\mathrm{~m}, 5 \mathrm{H})$, $5.24(\mathrm{ABq}, J=12.3 \mathrm{~Hz}, 2 \mathrm{H}), 3.66(\mathrm{ddd}, J=11.1,9.1,2.5 \mathrm{~Hz}, 1 \mathrm{H}), 3.42(\mathrm{~s}, 1 \mathrm{H}), 1.97(\mathrm{~d}, J=9.1$ $\mathrm{Hz}, 1 \mathrm{H}), 1.73-1.60(\mathrm{~m}, 1 \mathrm{H}), 1.47-1.37(\mathrm{~m}, 1 \mathrm{H}), 1.35(\mathrm{~s}, 3 \mathrm{H}), 1.01(\mathrm{t}, J=7.4 \mathrm{~Hz}, 3 \mathrm{H}) .{ }^{13} \mathrm{C} \mathrm{NMR}$ $\left(101 \mathrm{MHz}, \mathrm{CDCl}_{3}\right) \delta 176.4,135.3,128.8,128.7,128.3,77.6,77.1,67.9,23.5,21.9,10.6$. HPLC analysis: Daicel Chiralpak AD-H, hexane/iso-propanol $=95: 5$, flow rate $=0.8 \mathrm{~mL} / \mathrm{min}, \lambda=209 \mathrm{~nm}$, retention time: 34.87 (minor) and 39.47 min (major). ESI-HR calcd for $\mathrm{C}_{13} \mathrm{H}_{18} \mathrm{O}_{4} \mathrm{Na}^{+}: 261.1097$, found 261.1080. IR (thin film, $\mathrm{cm}^{-1}$ ): 3019, 1730, 1214, 744, 668;

Chemoselective acylation with propionic anhydride: To a solution of $\mathbf{8 b}$ in $\mathrm{CH}_{2} \mathrm{Cl}_{2}(2.0 \mathrm{~mL})$ was added DMAP (2.3 mg, $0.02 \mathrm{mmol}, 0.1$ equiv), $\mathrm{Et}_{3} \mathrm{~N}$ ( $26 \mathrm{uL}, 0.2 \mathrm{mmol}, 1$ equiv), and propionic anhydride ( $25 \mathrm{uL}, 0.2 \mathrm{mmol}, 1$ equiv). The reaction was stirred for 2 hours at room temperature, quenched with saturated aqueous $\mathrm{NaHCO}_{3}$, and stirred for an additional 30 minutes. The reaction mixture was extracted with $\mathrm{CH}_{2} \mathrm{Cl}_{2}(4 \times 2 \mathrm{~mL})$. The organic layers were combined, dried over $\mathrm{Na}_{2} \mathrm{SO}_{4}$, and concentrated. The residue was purified by column chromatography to obtain $\mathbf{9 b}$ $(94 \%$ yield, $96 \%$ ee $)$ as a colorless oil. $[\alpha]_{\mathrm{D}}{ }^{26}=-19.74\left(\mathrm{c}=1.6, \mathrm{CHCl}_{3}\right) ;{ }^{1} \mathbf{H} \mathbf{~ N M R}(400 \mathrm{MHz}$, $\left.\mathrm{CDCl}_{3}\right) \delta 7.47-7.30(\mathrm{~m}, 5 \mathrm{H}), 5.12(\mathrm{ABq}, 12.2 \mathrm{~Hz}, 2 \mathrm{H}), 5.11(\mathrm{dd}, \mathrm{J}=9.7,3.6 \mathrm{~Hz}, 1 \mathrm{H}), 3.26(\mathrm{~s}$, $1 \mathrm{H}), 2.20(\mathrm{dtt}, J=23.8,16.4,7.6 \mathrm{~Hz}, 2 \mathrm{H}), 1.75-1.63(\mathrm{~m}, 2 \mathrm{H}), 1.38(\mathrm{~s}, 3 \mathrm{H}), 1.07(\mathrm{t}, J=7.6 \mathrm{~Hz}$, 3H), $0.89(\mathrm{t}, J=7.4 \mathrm{~Hz}, 3 \mathrm{H}) .{ }^{13} \mathbf{C} \mathbf{N M R}\left(101 \mathrm{MHz}, \mathrm{CDCl}_{3}\right) \delta 175.2,173.8,135.1,128.8,128.70$, 128.70, 78.0, 76.5, 68.0, 27.6, 21.7, 21.4, 10.3, 9.4. HPLC analysis (>20:1 dr for racemic compound): Daicel Chiralpak OJ-H, hexane/iso-propanol $=95: 5$, flow rate $=1.0 \mathrm{~mL} / \mathrm{min}, \lambda=244$ nm, retention time: 8.07 (minor) and 9.49 min (major). ESI-HR calcd for $\mathrm{C}_{16} \mathrm{H}_{22} \mathrm{O}_{5} \mathrm{Na}^{+}$: 317.1359, found 317.1347. IR (thin film, $\mathrm{cm}^{-1}$ ): 3019, 1733, 1214, 929, 909, 744, 668 . 


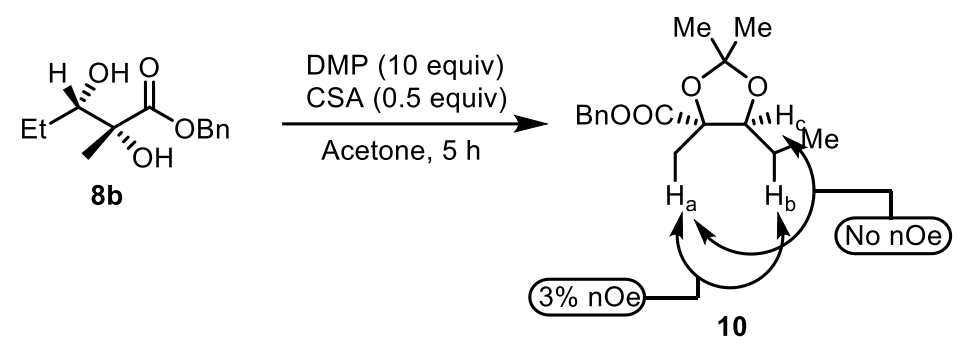

Determination of absolute and relative stereochemistry: A solution of $\mathbf{8 b}(44.4 \mathrm{mg}, 0.2 \mathrm{mmol})$ in 5 $\mathrm{mL}$ of acetone was added 2,2-dimethoxypropane (DMP) (10 equiv, $0.25 \mathrm{ml}$ ) and camphorsulfonic acid (CSA) $(23.2 \mathrm{mg}, 0.10 \mathrm{mmol}, 0.50$ equiv) at room temperature and stirred for $5 \mathrm{~h}$. The reaction was quenched with saturated $\mathrm{NaHCO}_{3}(10 \mathrm{~mL})$. The aqueous layer was extracted with 15 $\mathrm{mL}$ of DCM 3 times. The combined organic layers were dried over anhydrous $\mathrm{Na}_{2} \mathrm{SO}_{4}$ and concentrated to provide pure $\mathbf{1 0}\left(53.4 \mathrm{mg}, 96 \%\right.$ yield) as a colorless liquid. ${ }^{1} \mathbf{H}$ NMR (400 MHz, $\left.\mathrm{CDCl}_{3}\right) \delta 7.38-7.26(\mathrm{~m}, 5 \mathrm{H}), 5.20(\mathrm{ABq}, J=12.3 \mathrm{~Hz}, 2 \mathrm{H}), 4.09(\mathrm{t}, J=6.6 \mathrm{~Hz}, 1 \mathrm{H}), 1.61(\mathrm{~m}, 2 \mathrm{H})$, $1.47(\mathrm{~s}, 3 \mathrm{H}), 1.38(\mathrm{~s}, 3 \mathrm{H}), 1.34$ (s, 3H), 0.99 (t, $J=7.4 \mathrm{~Hz}, 3 \mathrm{H}) .{ }^{13} \mathbf{C} \mathbf{N M R}\left(101 \mathrm{MHz}, \mathrm{CDCl}_{3}\right) \delta$ 173.3, 135.8, 128.7, 128.4, 128.2, 109.0, 82.7, 81.9, 67.1, 28.4, 25.6, 23.0, 19.8, 11.2. ESI-HR calcd for $\mathrm{C}_{16} \mathrm{H}_{22} \mathrm{O}_{5} \mathrm{Na}^{+}: 301.1410$, found 301.1409 . The stereochemistry of the reduced product $\mathbf{8 b}$ was confirmed by an nOe experiment of the protected diols 10. Proton (Ha) was irradiated and $3 \%$ NOE correlation to Hb, while no correlation to Hc was observed (Supplementary Fig. 1). 

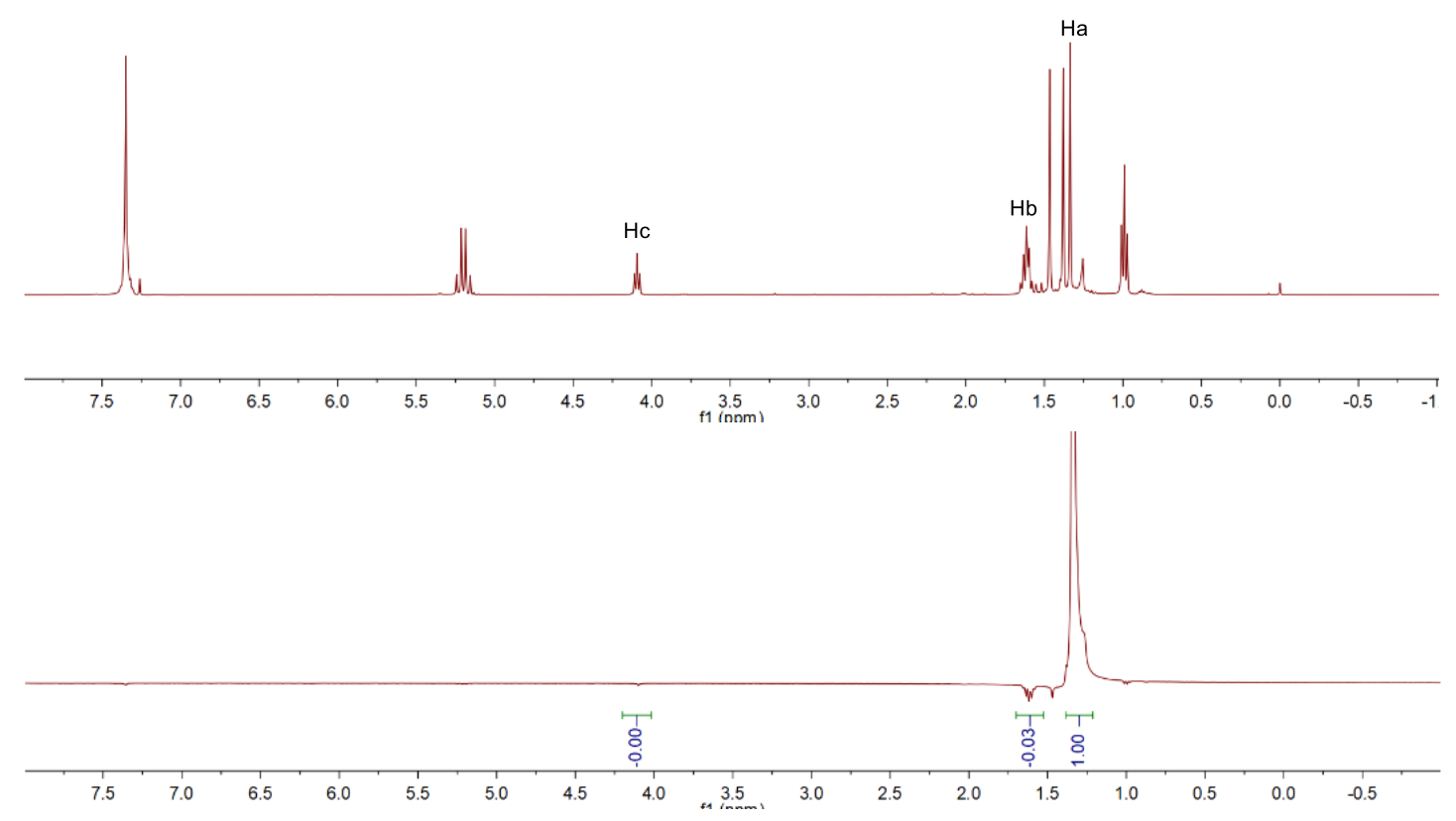

Supplementary Figure 1. nOe Experiment of the protected diols 10.

\section{Isotopic Lablling}

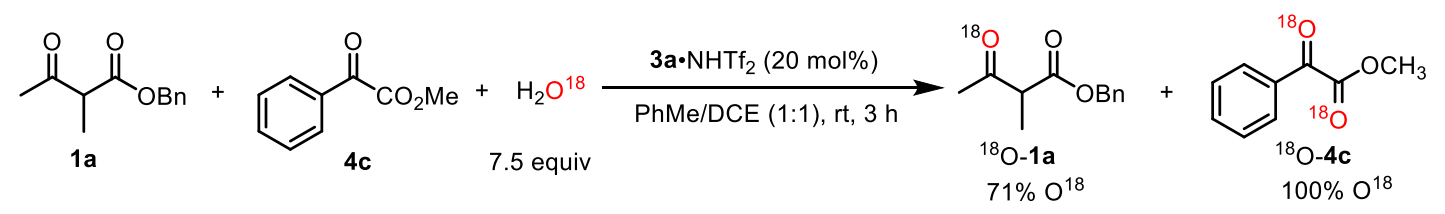

An oven-dried tube equipped with stir bar was charged with corresponding $\beta$-ketocarbonyl 1a $(0.2$ mmol) and aminocatalyst 3a/NHTf 2 (20 mol\%). After dissolution in mixed solvent of toluene and 1,2-dichloroethane $(0.5 \mathrm{~mL})$, Methyl benzoylformate $4 \mathbf{c}(20 \mathrm{~mol} \%)$ was added to the vial. Then $\mathrm{H}_{2} \mathrm{O}^{18}(30 \mu \mathrm{L}, 15 \mathrm{mmol})$ was added via syringe. Upon completion of the addition, the reaction was conducted at room temperature for 4 hours. The ratio of ${ }^{16} \mathrm{O} /{ }^{18} \mathrm{O}$ in the $\beta$-ketocarbonyl and ketone catalyst corresponding to starting material were analyzed by mass spectroscopy. Monitored by ESI-MS, ${ }^{18} \mathrm{O}$ was incorporated into the $\beta$-ketocarbonyls affording mixture of ${ }^{16} \mathrm{O}-1 \mathbf{a}$ and ${ }^{18} \mathrm{O}-\mathbf{1 a}$ with the ratio 29:71 (Supplementary Fig. 2). The completely double ${ }^{18} \mathrm{O}-$ label of $\mathbf{4 c}$ was observed to get ${ }^{18} \mathrm{O}-\mathbf{4 c}$. 

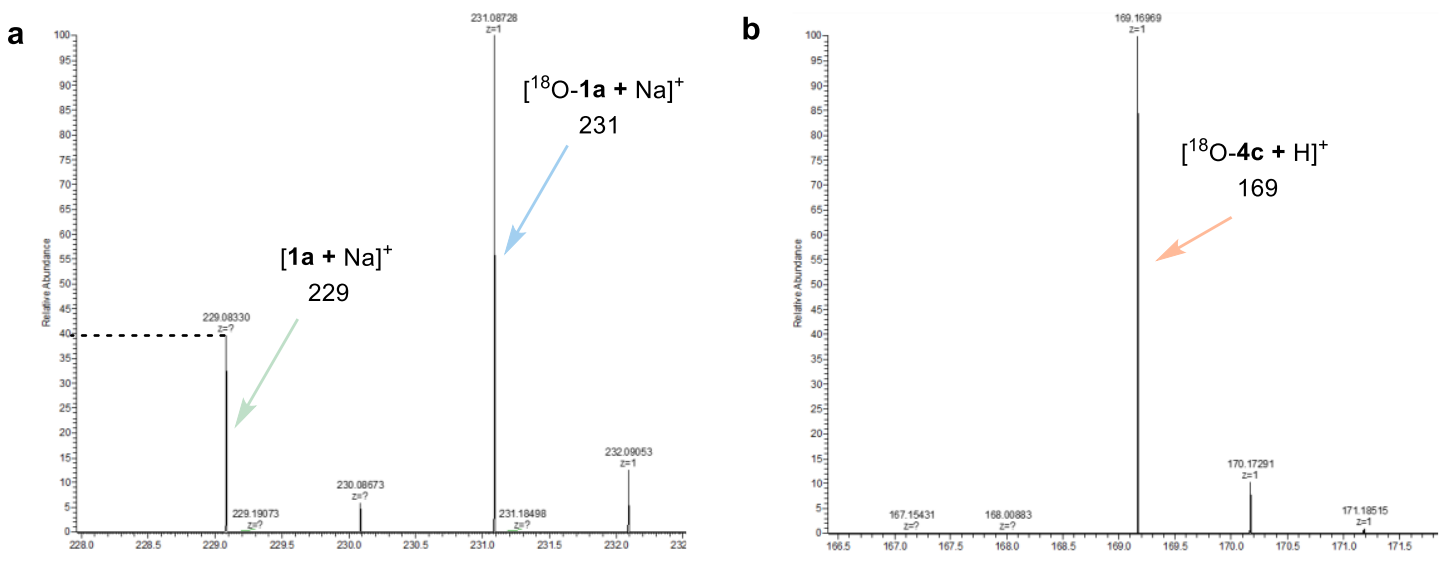

Supplementary Figure 2. a, ESI-MS of 1a under the catalysis of 3a and $\mathbf{4} \mathbf{c}$ without addition of $\mathrm{H}_{2} \mathrm{O}_{2} ; \mathbf{b}$, ESI-MS of $\mathbf{4} \mathbf{c}$ under the catalysis of $\mathbf{3 a}$ and existence of $\mathbf{4 a}$ without addition of $\mathrm{H}_{2} \mathrm{O}_{2}$.

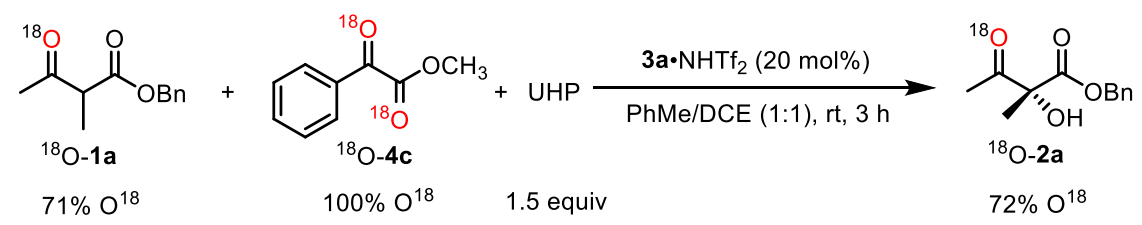

The ratio of ${ }^{18} \mathrm{O}-\mathbf{1 a}$ and ${ }^{18} \mathrm{O}-\mathbf{4} \mathbf{c}$ remained stable with the extension of reaction time. UHP, a complex of hydrogen peroxide and urea, was added in situ after 3 hours. The reaction was continued for another 3 hours, and the ratio of isotopic labelling of $1 \mathbf{a}$ and $\mathbf{4 c}$ did not change. The ratio of ${ }^{16} \mathrm{O} /{ }^{18} \mathrm{O}$ in the product $\mathbf{2 a}$ is close to $\mathbf{1 a}(28: 72)$, with no signal of double ${ }^{18} \mathrm{O}$-labelling (Supplementary Fig. 3).

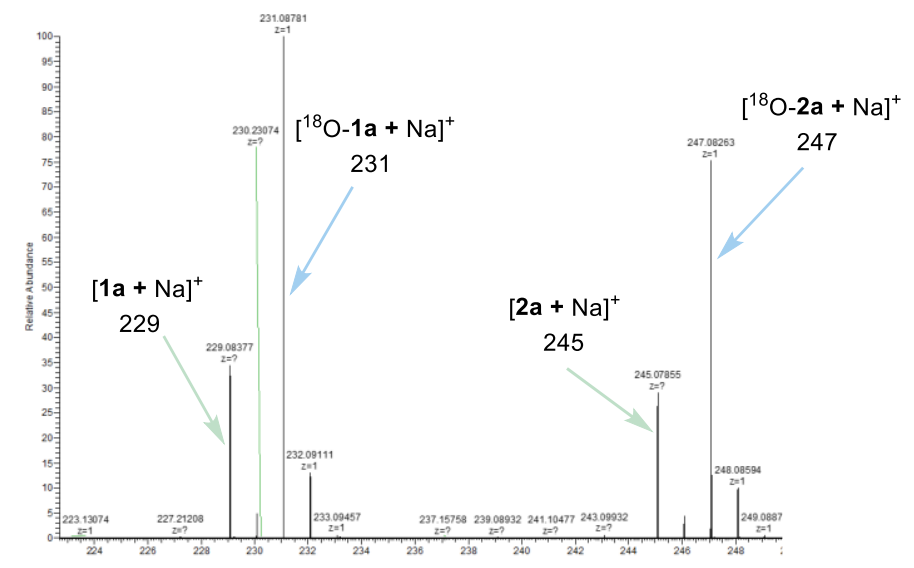


Supplementary Figure 3. ESI-MS of $1 \mathbf{a}$ and $\mathbf{2 a}$ under the catalysis of $\mathbf{3 a}$ and $\mathbf{4} \mathbf{c}$ after addition of $\mathrm{H}_{2} \mathrm{O}_{2}$.

\section{Kinetics Study}

For the biphasic system (dichloromethane/toluene/ $\mathrm{H}_{2} \mathrm{O}$ ), the actual concentration of $\mathrm{H}_{2} \mathrm{O}_{2}$ in organic phase is determined only by partition coefficient under the premise that mass transfer of the reagents back and forth between the phases is not the rate-determining step (rds). In view of the endeavor of Strukul and Ding ${ }^{14-15}$, the stirring rate was fixed to $1000 \mathrm{rpm}$ which the plot is reproducible in reaction profiles (Supplementary Fig. 4). And the amount of hydrogen peroxide present in the whole system used in "excess" has no effect on the results. The kinetic data was obtained by measuring the initial rate in different concentration of $\mathrm{H}_{2} \mathrm{O}_{2}$, substrate and catalysts (Supplementary Table 6-9).

\section{Mass Transfer Effect}

To the reaction apparatus was added $\beta$-ketocarbonyls $1 \mathbf{a}(0.2 \mathrm{mmol})$ and aminocatalyst $3 \mathbf{a} / \mathrm{NHTf}_{2}$ (20 mol\%). After dissolution in mixed solvent of toluene and 1,2-dichloroethane $(0.5 \mathrm{~mL})$, trifluoroacetophenone $4 \mathbf{a}(20 \mathrm{~mol} \%)$ was added to the vial. The reaction was conducted under different stirring rates (300-1200 rpm). The reactions were monitored by GC in situ after the addition of $\mathrm{H}_{2} \mathrm{O}_{2}$ (30 wt.\% in water, $0.3 \mathrm{mmol}$ ) via syringe. The conversions were plotted vs. T measured for each reaction (Supplementary Fig. 4). 


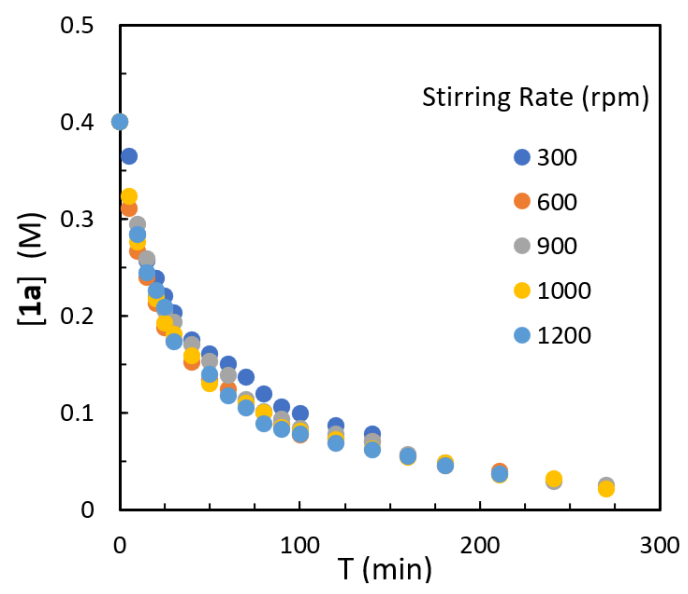

Supplementary Figure 4. Reaction profiles under different stirring rates.

\section{Substrate Dependence}

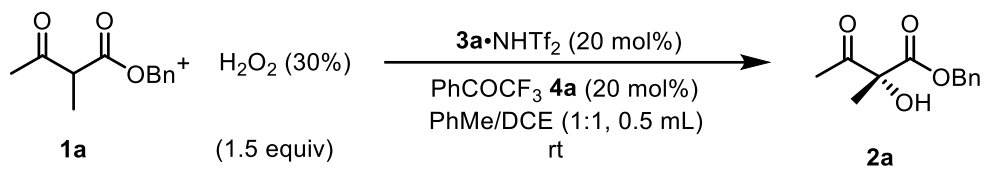

To the reaction apparatus was added $\beta$-ketocarbonyls 1a $(0.18-0.27 \mathrm{mmol})$ and aminocatalyst 3a/NHTf $2(20 \mathrm{~mol} \%)$. After dissolution in mixed solvent of toluene and 1,2-dichloroethane $(0.5$ $\mathrm{mL})$, trifluoroacetophenone $4 \mathbf{a}(20 \mathrm{~mol} \%)$ was added to the vial under the stirring rates of 1000 rpm. The reactions were monitored by GC in situ after the addition of $\mathrm{H}_{2} \mathrm{O}_{2}(30 \mathrm{wt} . \%$ in water, 0.3 mmol) via syringe. The rate conversions from $1-10 \%$ were measured for each reaction (Supplementary Table 6). These rates were plotted vs. [1a].

Supplementary Table 6. Rates of hydroxylation at differing [1a] 


\begin{tabular}{ccc}
\hline entry & {$[1 \mathrm{a}](\mathrm{M})$} & rate $(\mathrm{M} / \mathrm{min})$ \\
\hline 1 & 0.35 & 0.0076 \\
2 & 0.40 & 0.0089 \\
3 & 0.45 & 0.0076 \\
4 & 0.50 & 0.0085 \\
5 & 0.55 & 0.0085 \\
\hline
\end{tabular}

\section{Oxidant Dependence}

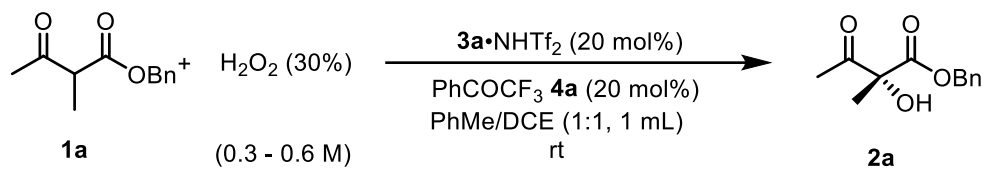

To the reaction apparatus was added $\beta$-ketocarbonyls $\mathbf{1 a}(0.2 \mathrm{mmol})$ and aminocatalyst $\mathbf{3 a} / \mathrm{NHTf}_{2}$ (20 mol\%). After dissolution in mixed solvent of toluene and 1,2-dichloroethane (1.0 mL), trifluoroacetophenone $4 \mathbf{a}(20 \mathrm{~mol} \%)$ was added to the vial under the stirring rates of $1000 \mathrm{rpm}$. The reactions were monitored by GC in situ after the addition of $\mathrm{H}_{2} \mathrm{O}_{2}(30$ wt.\% in water, 0.3-0.6 M) via syringe. The rate conversions from 1-10\% were measured for each reaction (Supplementary Table 7). These rates were plotted vs. $\left[\mathrm{H}_{2} \mathrm{O}_{2}\right]$.

Supplementary Table 7. Rates of hydroxylation at differing $\left[\mathrm{H}_{2} \mathrm{O}_{2}\right]$

\begin{tabular}{ccc}
\hline entry & {$\left[\mathrm{H}_{2} \mathrm{O}_{2}\right](\mathrm{M})$} & rate $(\mathrm{M} / \mathrm{min})$ \\
\hline 1 & 0.30 & 0.0057 \\
2 & 0.40 & 0.0054 \\
3 & 0.50 & 0.0052 \\
4 & 0.60 & 0.0053 \\
\hline
\end{tabular}

\section{Aminocatalyst Dependence}




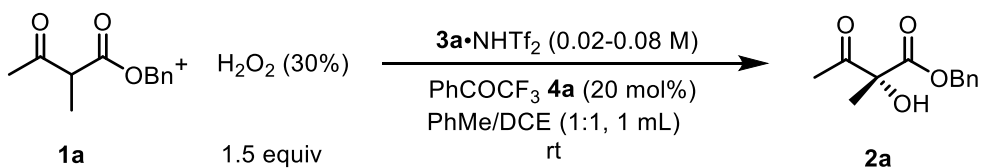

To the reaction apparatus was added $\beta$-ketocarbonyls $\mathbf{1 a}(0.2 \mathrm{mmol})$ and aminocatalyst $\mathbf{3 a} / \mathrm{NHTf}_{2}$ $(0.02-0.08 \mathrm{M})$. After dissolution in mixed solvent of toluene and 1,2-dichloroethane $(0.5 \mathrm{~mL})$, trifluoroacetophenone $4 \mathbf{a}(20 \mathrm{~mol} \%)$ was added to the vial under the stirring rates of $1000 \mathrm{rpm}$. The reactions were monitored by GC in situ after the addition of $\mathrm{H}_{2} \mathrm{O}_{2}$ (30 wt.\% in water, 0.3 mmol) via syringe. The rate conversions from 1-10\% were measured for each reaction (Supplementary Table 8). These rates were plotted vs. [3a].

Supplementary Table 8. Rates of hydroxylation at differing [3a]

\begin{tabular}{ccc}
\hline entry & {$[3 \mathrm{a}](\mathrm{M})$} & rate $(\mathrm{M} / \mathrm{min})$ \\
\hline 1 & 0.20 & 0.0030 \\
2 & 0.30 & 0.0052 \\
3 & 0.50 & 0.0062 \\
4 & 0.60 & 0.0075 \\
5 & 0.80 & 0.0089 \\
\hline
\end{tabular}

\section{Ketone catalyst Dependence}

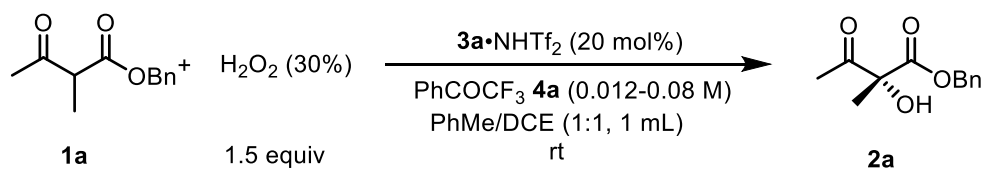

To the reaction apparatus was added $\beta$-ketocarbonyls $\mathbf{1 a}(0.2 \mathrm{mmol})$ and aminocatalyst $3 \mathbf{a} / \mathrm{NHTf}_{2}$ (0.02-0.08 M). After dissolution in mixed solvent of toluene and 1,2-dichloroethane $(0.5 \mathrm{~mL})$, trifluoroacetophenone $4 \mathbf{a}(0.012-0.08 \mathrm{M})$ was added to the vial under the stirring rates of 1000 rpm. The reactions were monitored by GC in situ after the addition of $\mathrm{H}_{2} \mathrm{O}_{2}(30$ wt. \% in water, 0.3 
mmol) via syringe. The rate conversions from 1-10\% were measured for each reaction (Supplementary Table 9). These rates were plotted vs. [4a].

Supplementary Table 9. Rates of hydroxylation at differing [4a]

\begin{tabular}{ccc}
\hline entry & {$[4 \mathrm{a}](\mathrm{M})$} & rate $(\mathrm{M} / \mathrm{min})$ \\
\hline 1 & 0.012 & 0.0028 \\
2 & 0.029 & 0.0038 \\
3 & 0.040 & 0.0057 \\
4 & 0.052 & 0.0066 \\
5 & 0.080 & 0.0089 \\
\hline
\end{tabular}




\section{Computational Investigations}

DFT calculations were performed with the Gaussian 09 program package ${ }^{16}$. All of the geometries were fully optimized at the B3LYP ${ }^{17-20} / 6-31 \mathrm{G}(\mathrm{d})$ level of theory, in conjunction with the IEF-PCM implicit solvation mode ${ }^{21}$ to account for the solvation

effects of mixed toluene and DCE (ratio = 1:1). All of the optimized geometries were verified by frequency computations as minima (zero imaginary frequencies) or transition structures (a single imaginary frequency). Single-point energy calculations on the optimized geometries were then evaluated using the dispersioncorrected density functional method B3LYP-D322 (with a Becke-Johnson (BJ) damping function ${ }^{23-25}$ ) and the triple-zeta valence quality def2-TZVPP basis set of Weigend and Ahlrichs ${ }^{26}$, within the IEF-PCM model (Toluene:DCE = 1:1). The thermal corrections evaluated from the unscaled vibrational frequencies at the B3LYP/6-31G(d) level on the optimized geometries were then added to the B3LYP-D3(BJ)/def2-TZVPP electronic energies to obtain the free energies. The graphs of the optimized structures were generated with CYLview ${ }^{27}$.

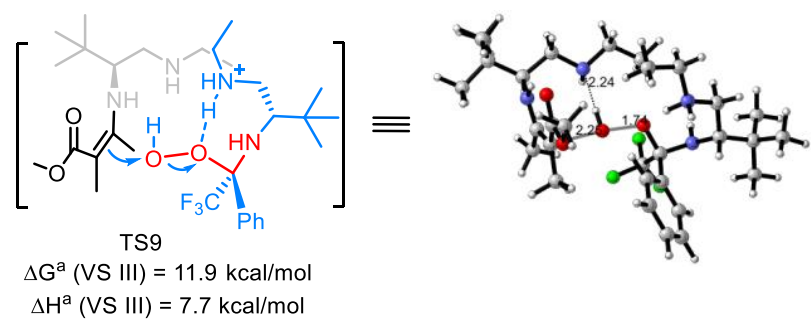

Supplementary Figure 5. The hydroxylation between protonated enamine Int3 and III. 
a

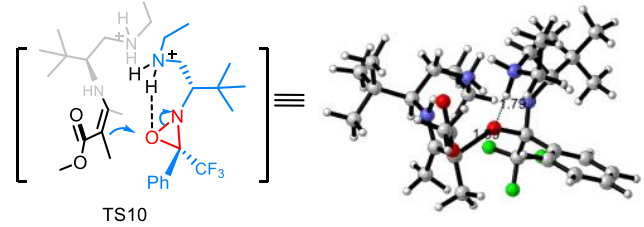

TS10

$\Delta \mathrm{G}^{\mathrm{a}}=40.9 \mathrm{kcal} / \mathrm{mo}$

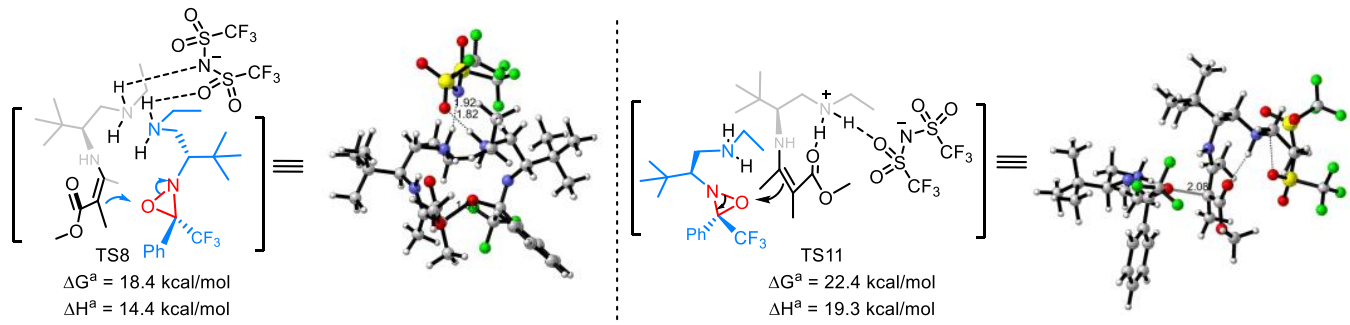

Supplementary Figure 6. The hydroxylation between protonated enamine Int3 and IV. a, The

direct coupling between two positive species. $\mathbf{b}$, The anion bridged coupling between enamine Int3 and IV. 


\section{Supplementary Figures}

1. Copies of NMR Spectra.

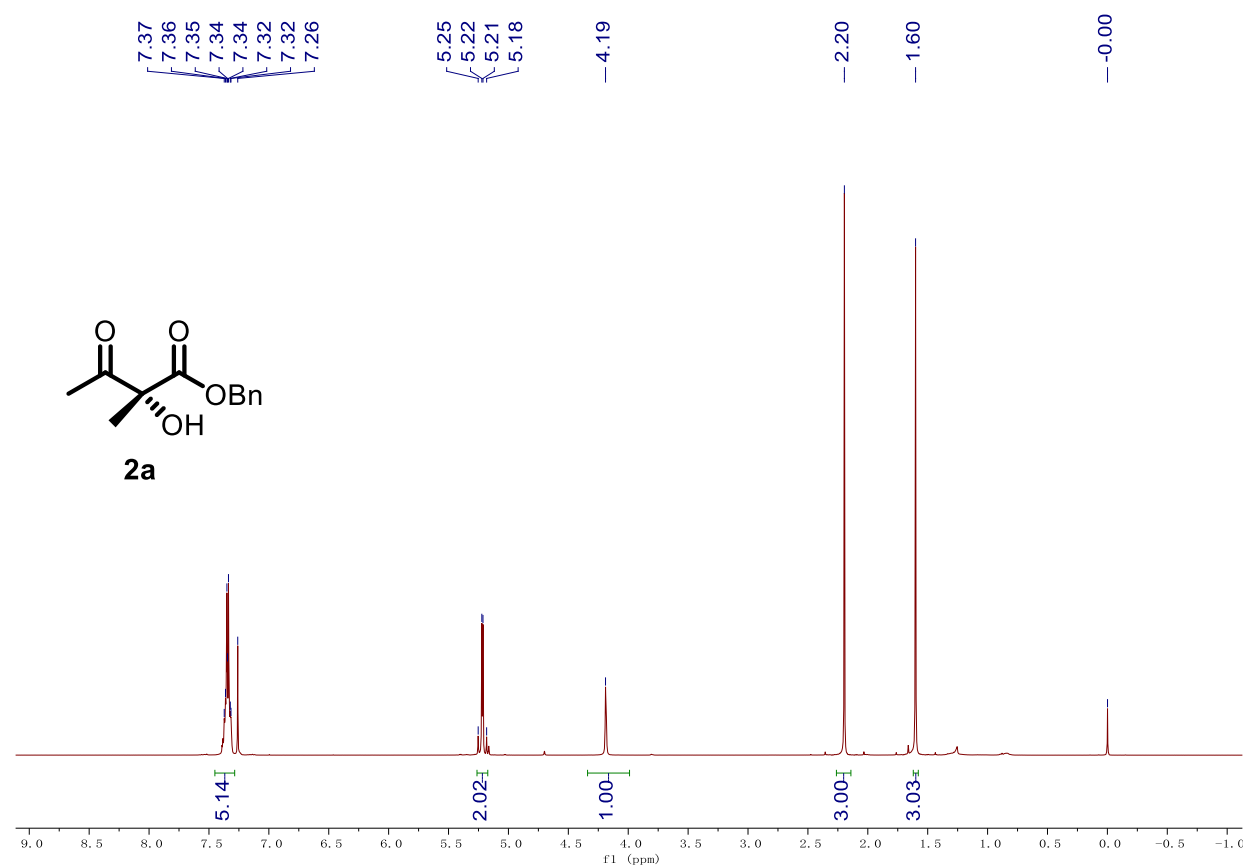

Figure S7. ${ }^{1} \mathrm{H}$ NMR $\left(400 \mathrm{MHz}, \mathrm{CDCl}_{3}\right)$ of $\mathbf{2 a}$.

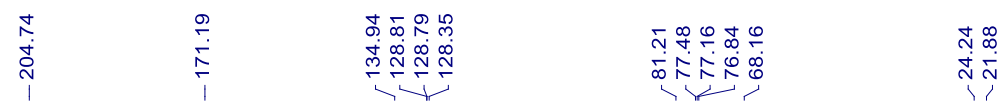

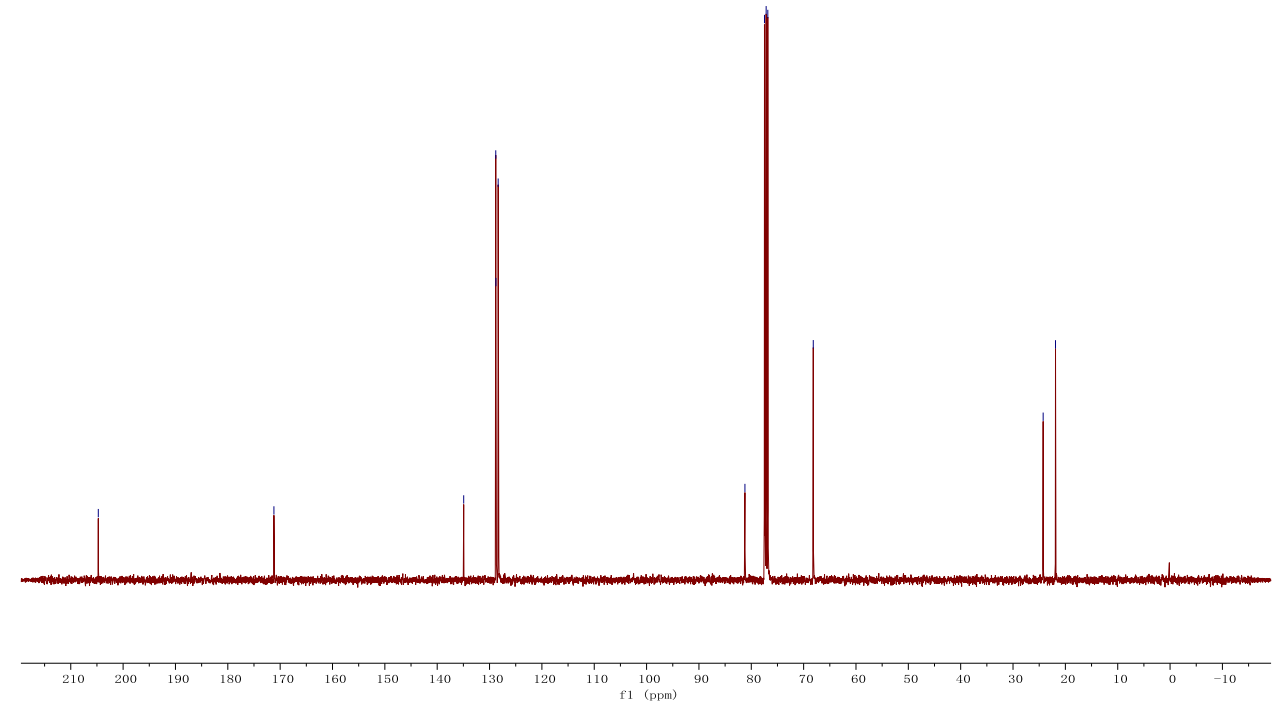

Figure S8. ${ }^{13} \mathrm{C}$ NMR $\left(100 \mathrm{MHz}, \mathrm{CDCl}_{3}\right)$ of $\mathbf{2 a}$. 


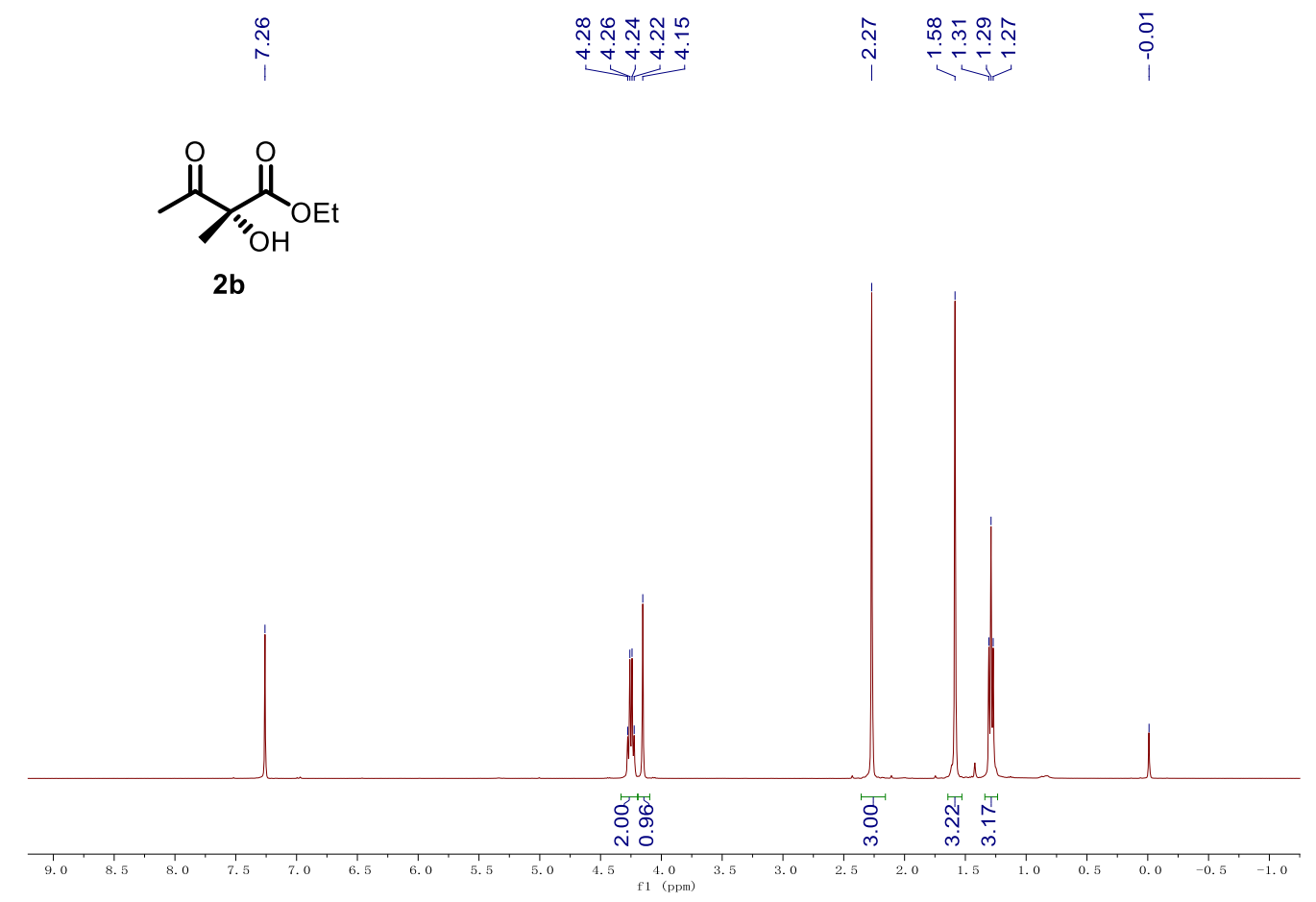

Figure S9. ${ }^{1} \mathrm{H}$ NMR (400 MHz, $\left.\mathrm{CDCl}_{3}\right)$ of $\mathbf{2 b}$.

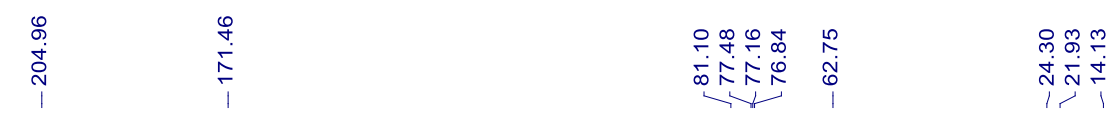

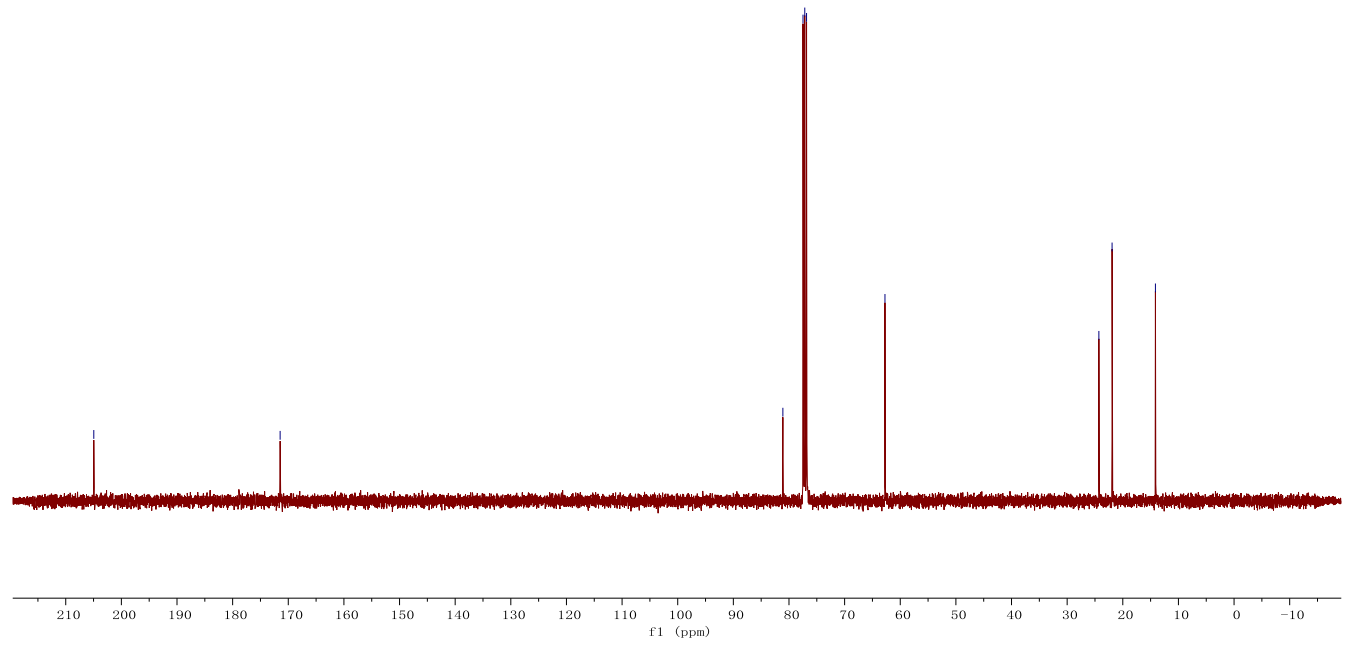

Figure S10. ${ }^{13} \mathrm{C}$ NMR $\left(100 \mathrm{MHz}, \mathrm{CDCl}_{3}\right)$ of $\mathbf{2 b}$. 


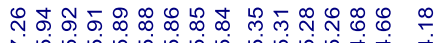

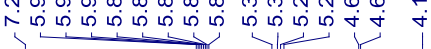

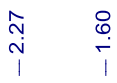

-
$\vdots$
$i$<smiles>C=CCOC(=O)[C@](C)(O)C(C)=O</smiles>

2c

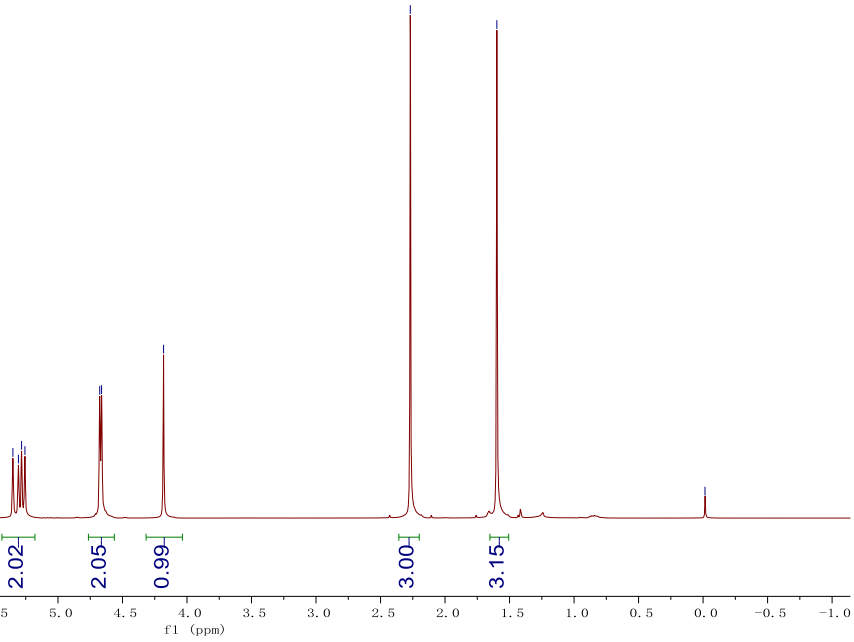

Figure S11. ${ }^{1} \mathrm{H}$ NMR $\left(400 \mathrm{MHz}, \mathrm{CDCl}_{3}\right)$ of $\mathbf{2 c}$.

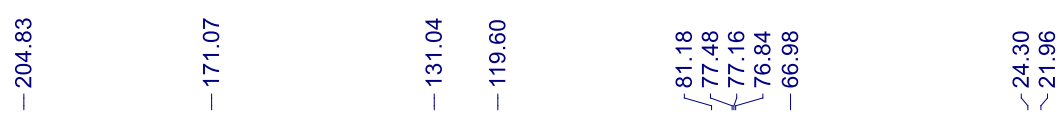
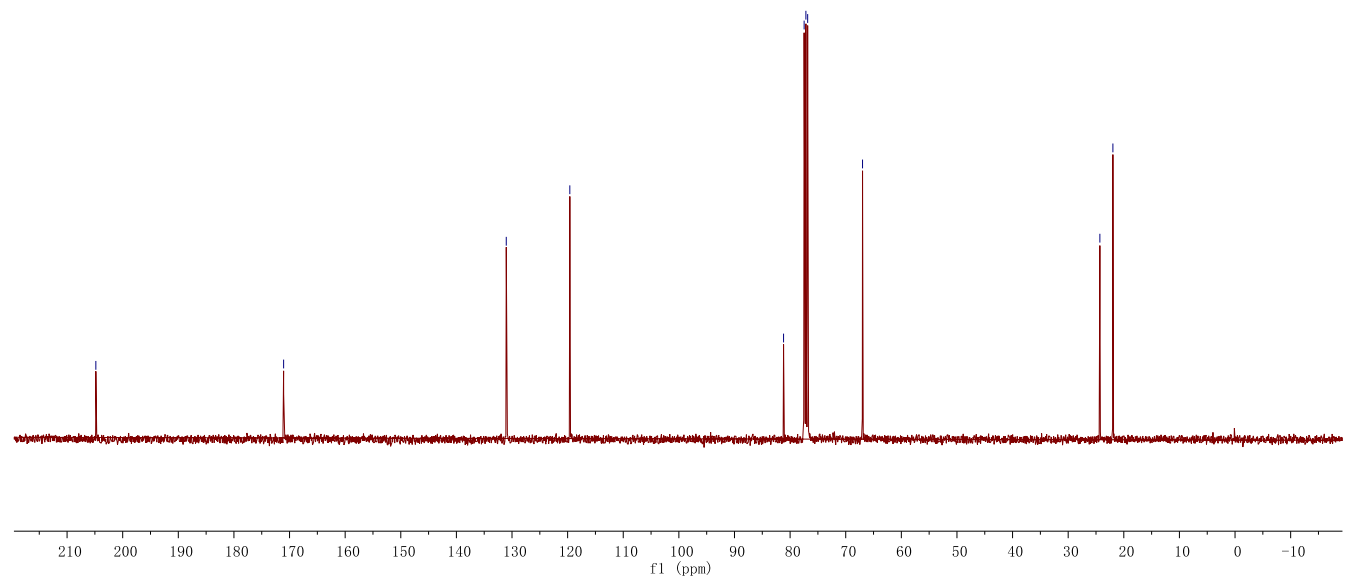

Figure S12. ${ }^{13} \mathrm{C}$ NMR $\left(100 \mathrm{MHz}, \mathrm{CDCl}_{3}\right)$ of $\mathbf{2 c}$. 


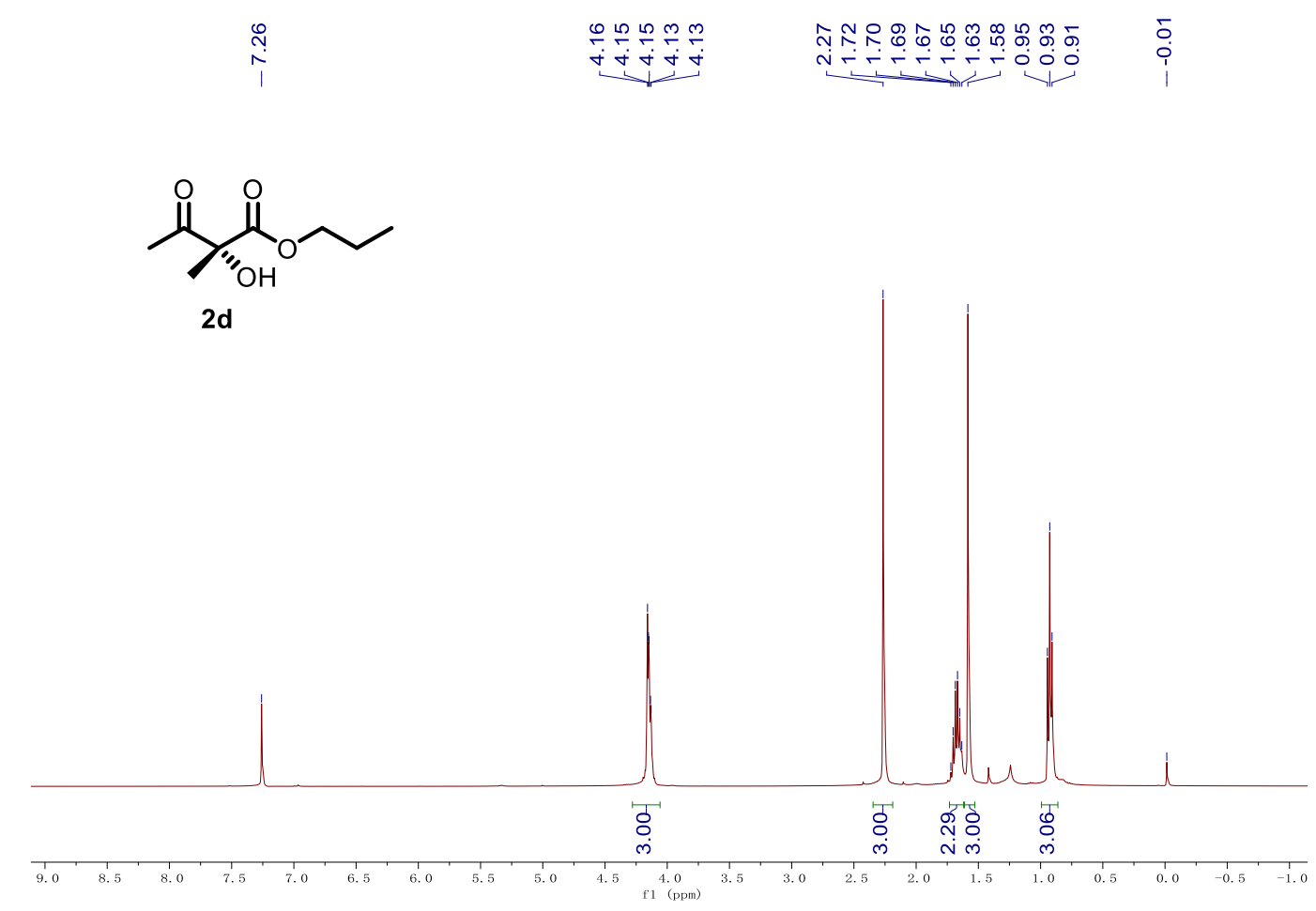

Figure S13. ${ }^{1} \mathrm{H}$ NMR $\left(400 \mathrm{MHz}, \mathrm{CDCl}_{3}\right)$ of $\mathbf{2 d}$.
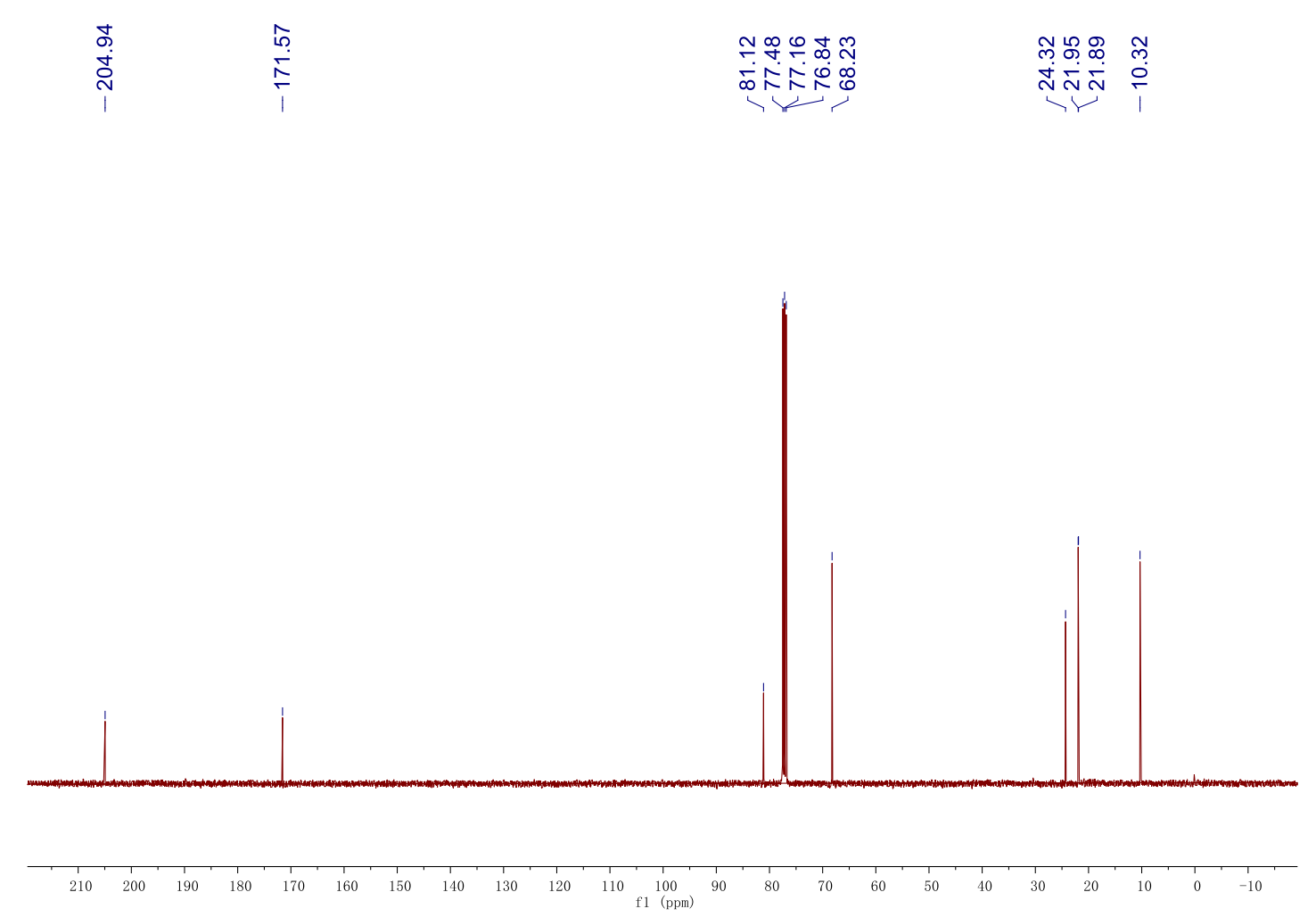

Figure S14. ${ }^{13} \mathrm{C}$ NMR $\left(100 \mathrm{MHz}, \mathrm{CDCl}_{3}\right)$ of $\mathbf{2 d}$. 


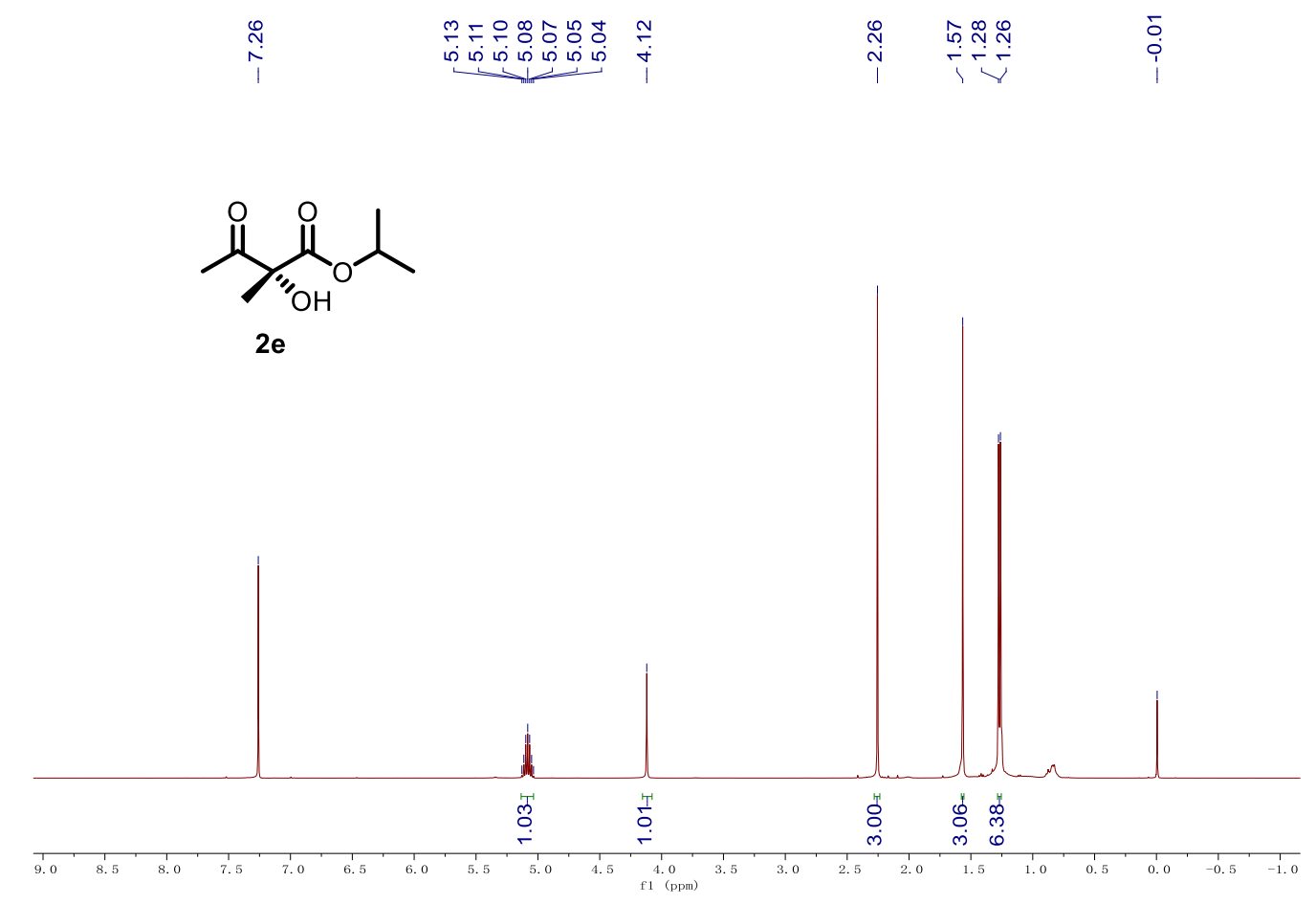

Figure S15. ${ }^{1} \mathrm{H}$ NMR $\left(400 \mathrm{MHz}, \mathrm{CDCl}_{3}\right)$ of $\mathbf{2 e}$.

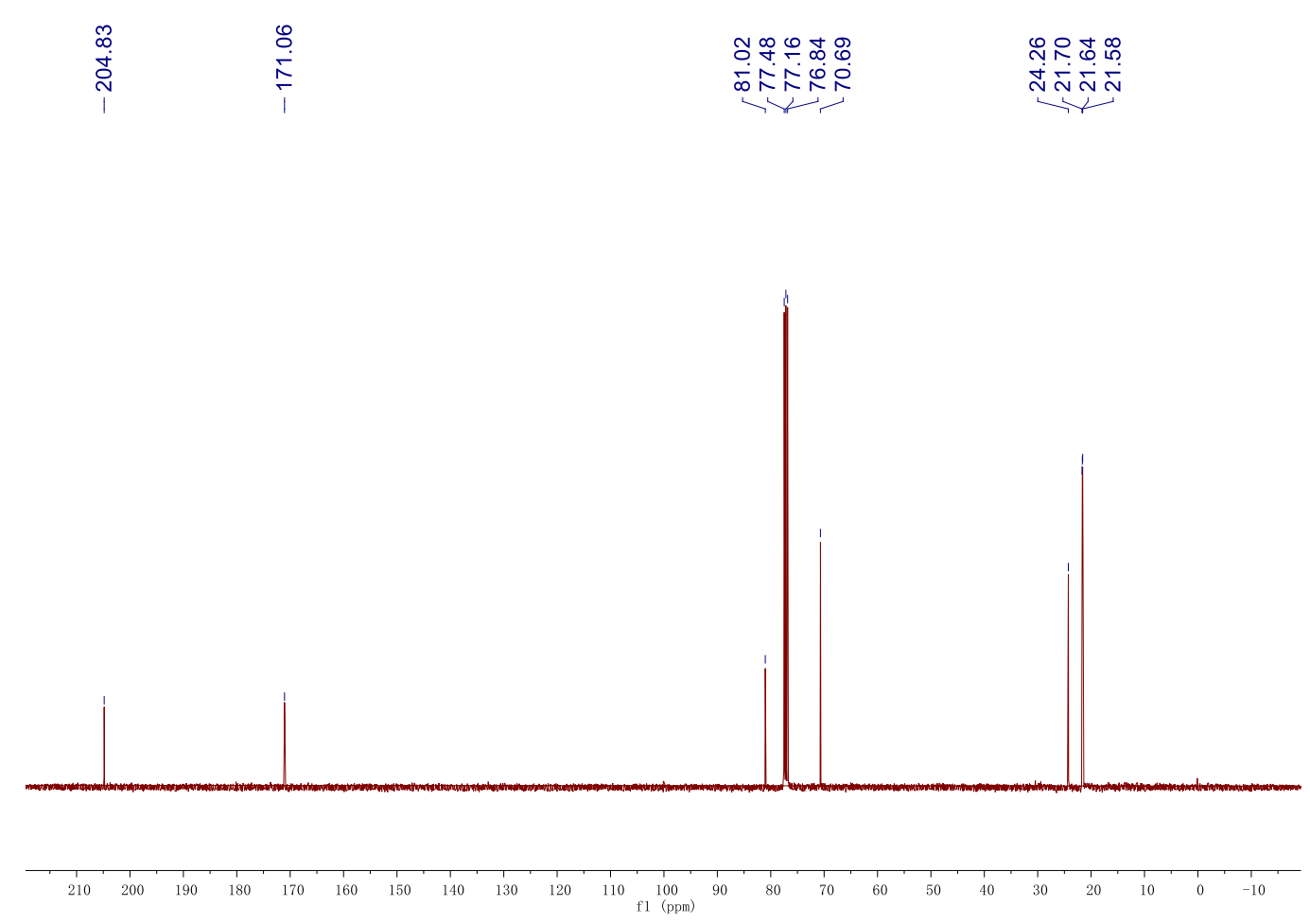

Figure S16. ${ }^{13} \mathrm{C}$ NMR $\left(100 \mathrm{MHz}, \mathrm{CDCl}_{3}\right)$ of $\mathbf{2 e}$. 
<smiles>CCCCOC(=O)[C@@](C)(O)C(C)=O</smiles>

$2 f$

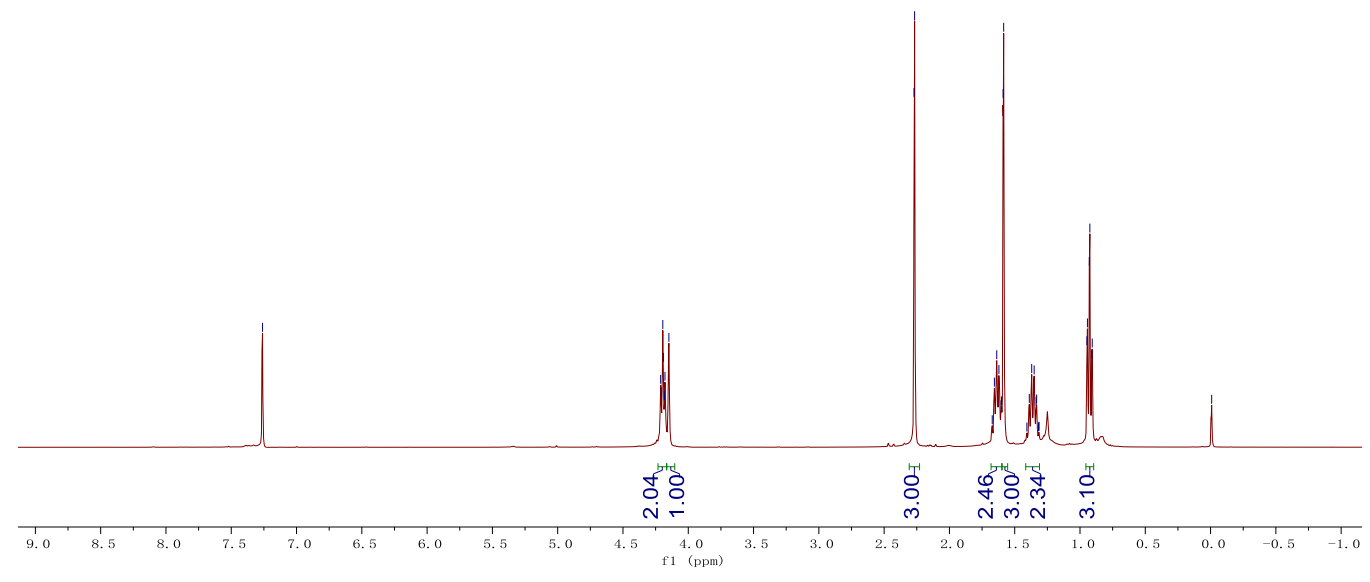

Figure S17. ${ }^{1} \mathrm{H}$ NMR $\left(400 \mathrm{MHz}, \mathrm{CDCl}_{3}\right)$ of $\mathbf{2 f}$.

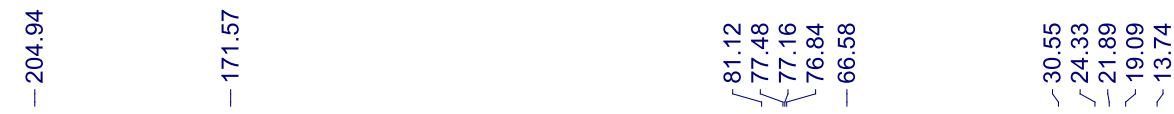

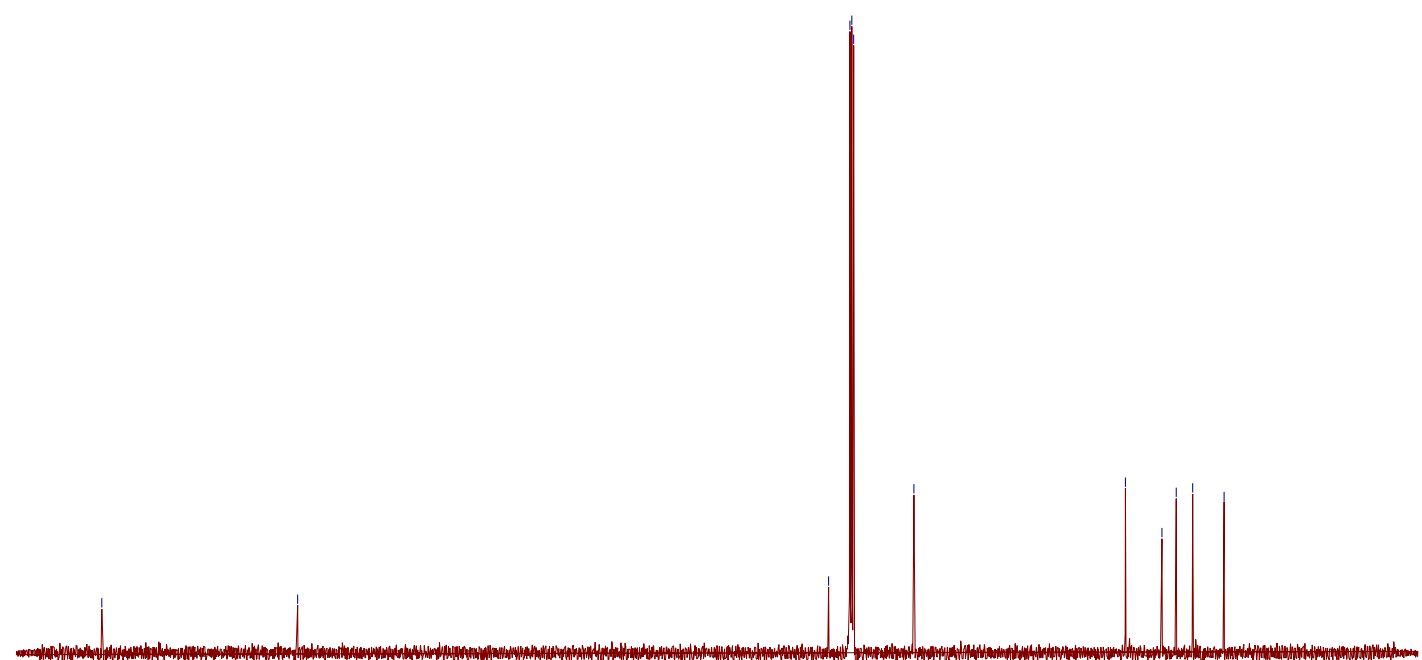

$\begin{array}{lllllllllllll}210 & 200 & 190 & 180 & 170 & 160 & 150 & 140 & 130 & 120 & 110 & 100 & 90 \\ \mathrm{f} 1(\mathrm{ppm}) & & & & & & \end{array}$

Figure S18. ${ }^{13} \mathrm{C}$ NMR $\left(100 \mathrm{MHz}, \mathrm{CDCl}_{3}\right)$ of $\mathbf{2 f}$. 
<smiles>CC(=O)[C@](C)(O)C(=O)OC/C=C/[PH2+]</smiles>

$2 \mathrm{~g}$

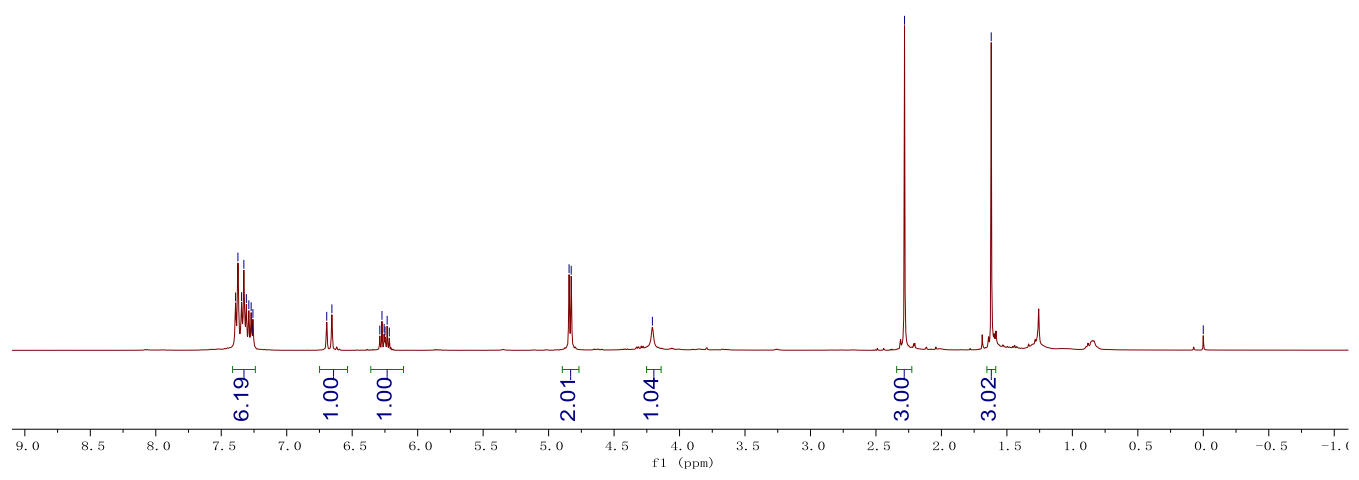

Figure S19. ${ }^{1} \mathrm{H} \mathrm{NMR}\left(400 \mathrm{MHz}, \mathrm{CDCl}_{3}\right)$ of $\mathbf{2 g}$.

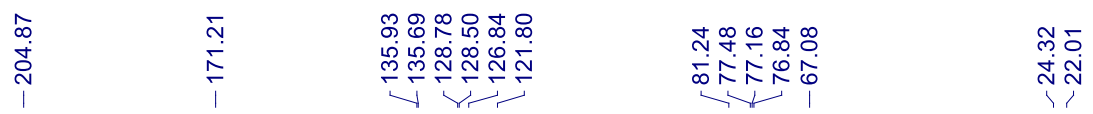

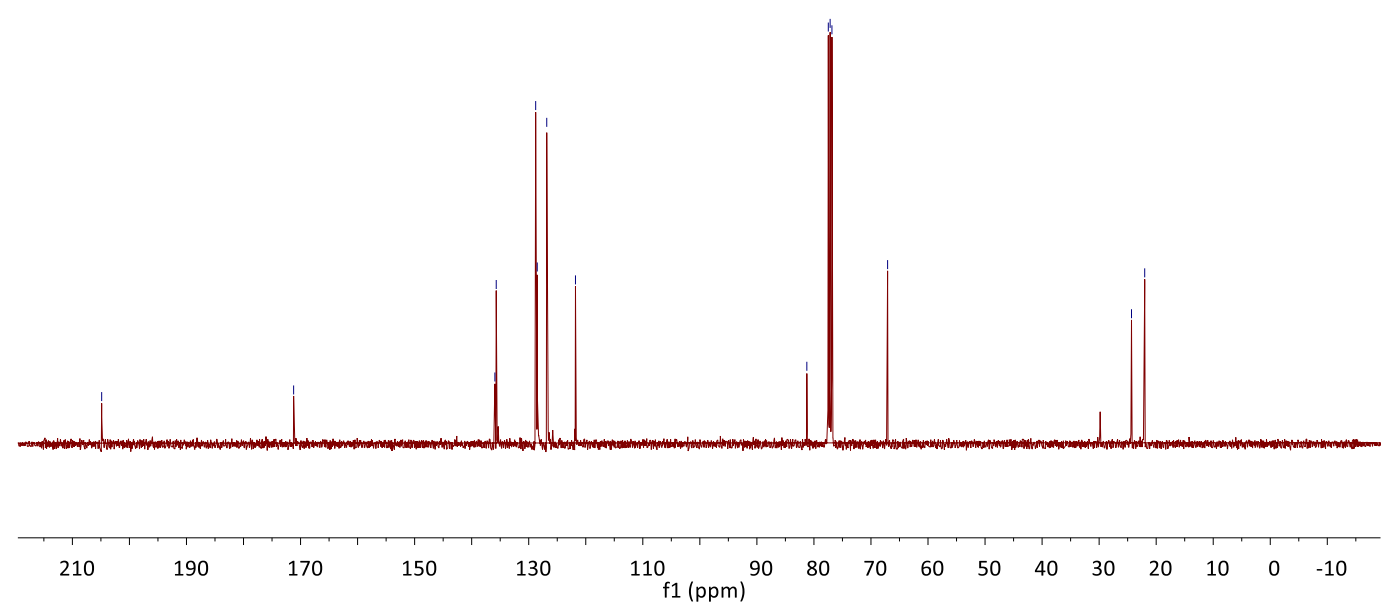

Figure S20. ${ }^{13} \mathrm{C}$ NMR $\left(100 \mathrm{MHz}, \mathrm{CDCl}_{3}\right)$ of $\mathbf{2 g}$. 
<smiles>CC(=O)[C@](C)(O)C(=O)OCc1cccc2ccccc12</smiles>

$2 \mathrm{~h}$

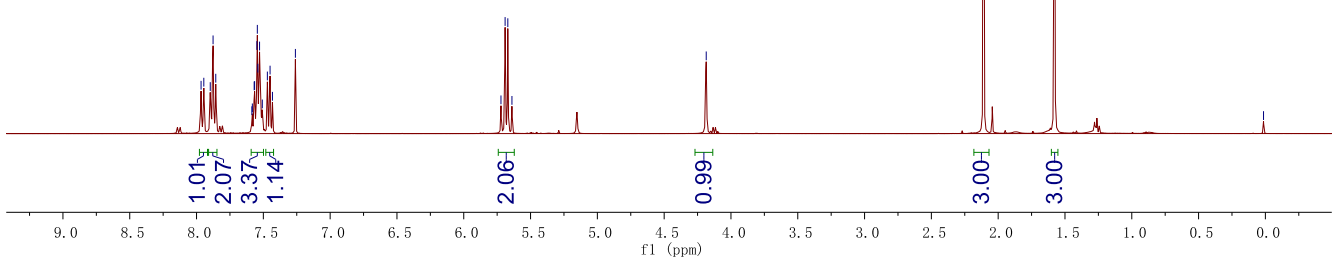

Figure S21. ${ }^{1} \mathrm{H}$ NMR $\left(400 \mathrm{MHz}, \mathrm{CDCl}_{3}\right)$ of $\mathbf{2 h}$.

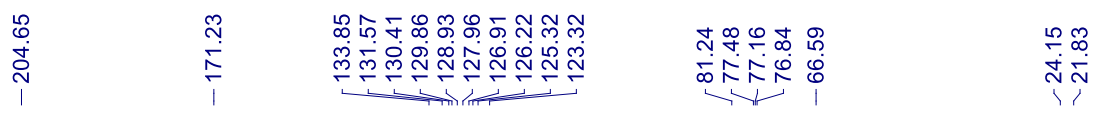

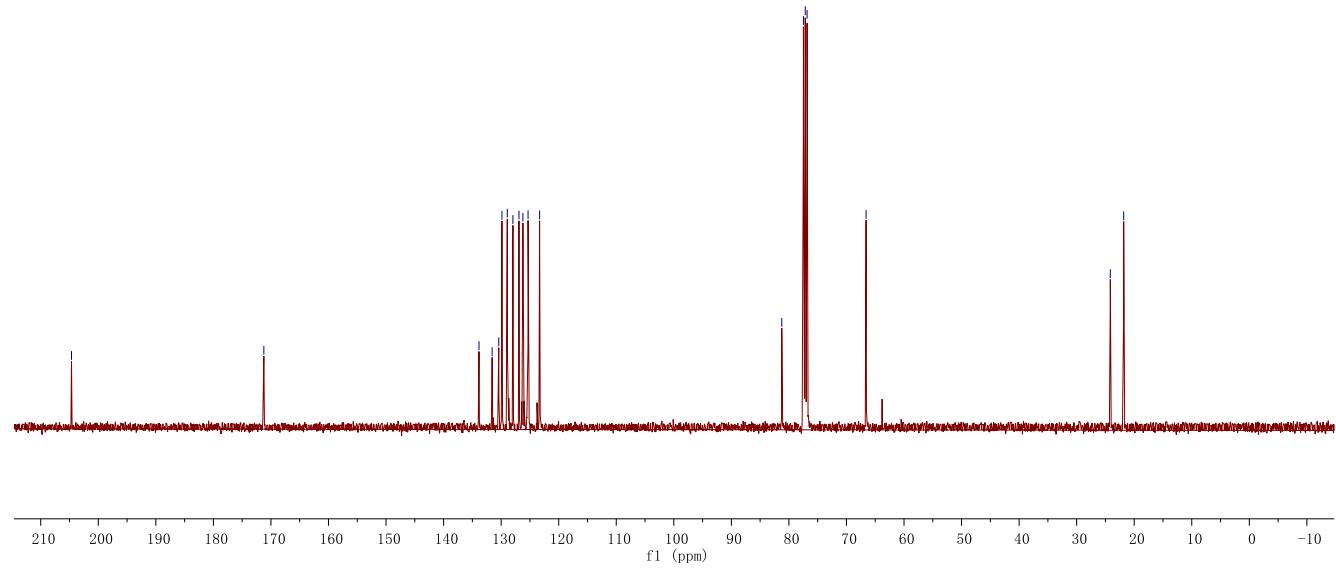

Figure S22. ${ }^{13} \mathrm{C}$ NMR $\left(100 \mathrm{MHz}, \mathrm{CDCl}_{3}\right)$ of $\mathbf{2 h}$. 

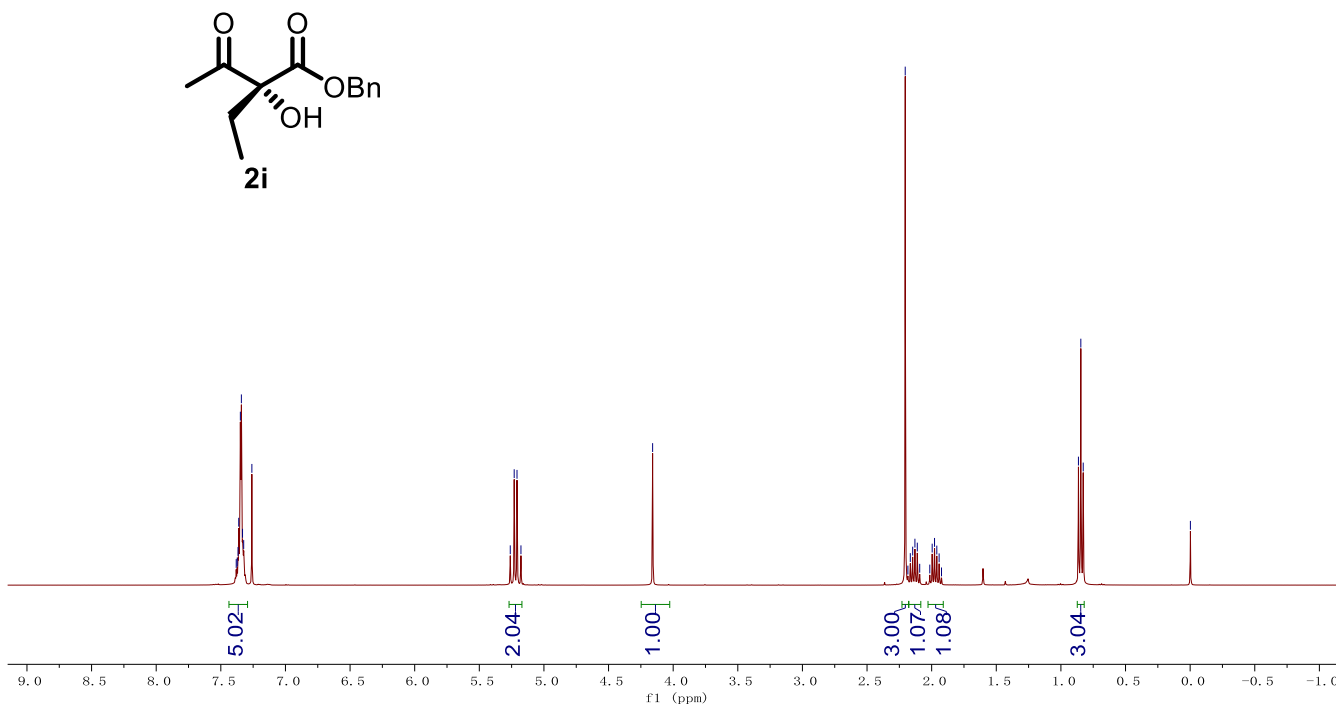

Figure S23. ${ }^{1} \mathrm{H}$ NMR $\left(400 \mathrm{MHz}, \mathrm{CDCl}_{3}\right)$ of $\mathbf{2 i}$.

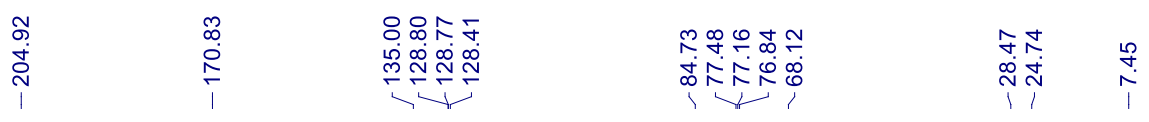

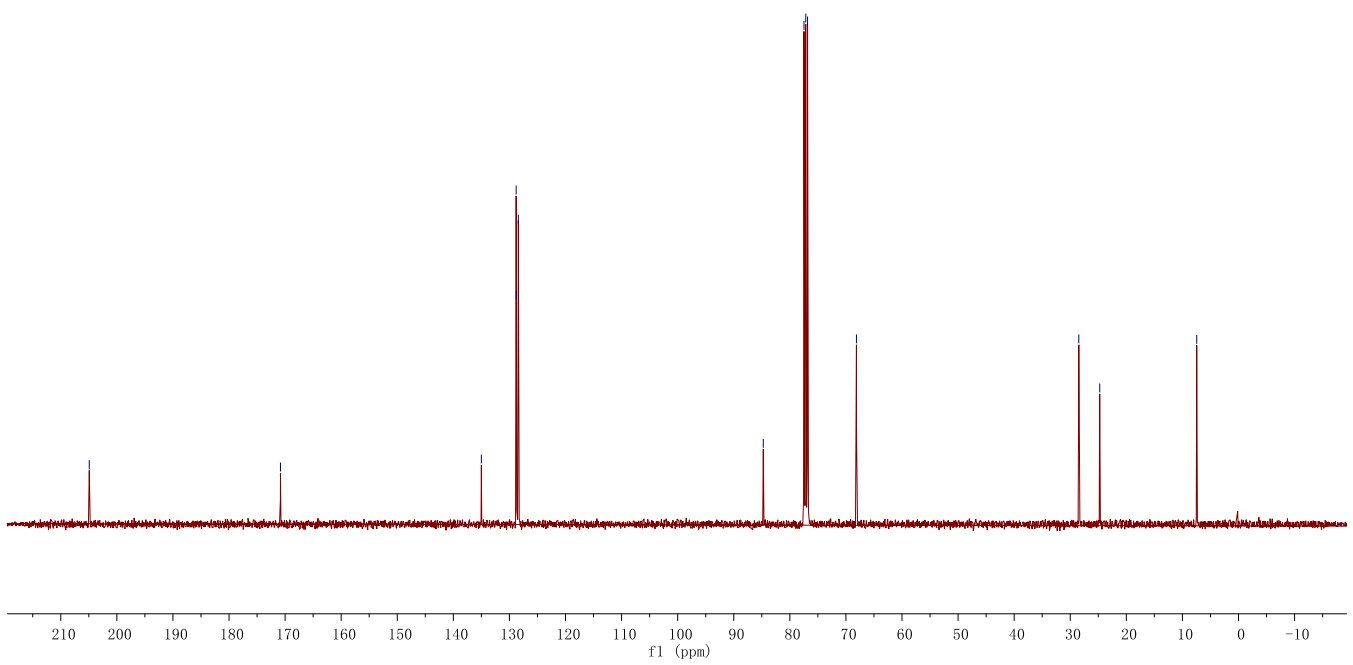

Figure S24. ${ }^{13} \mathrm{C}$ NMR $\left(100 \mathrm{MHz}, \mathrm{CDCl}_{3}\right)$ of $\mathbf{2 i}$. 

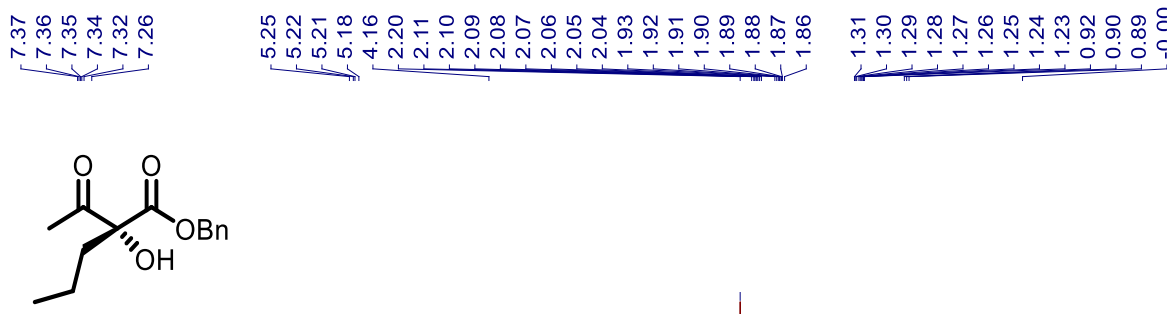

2j

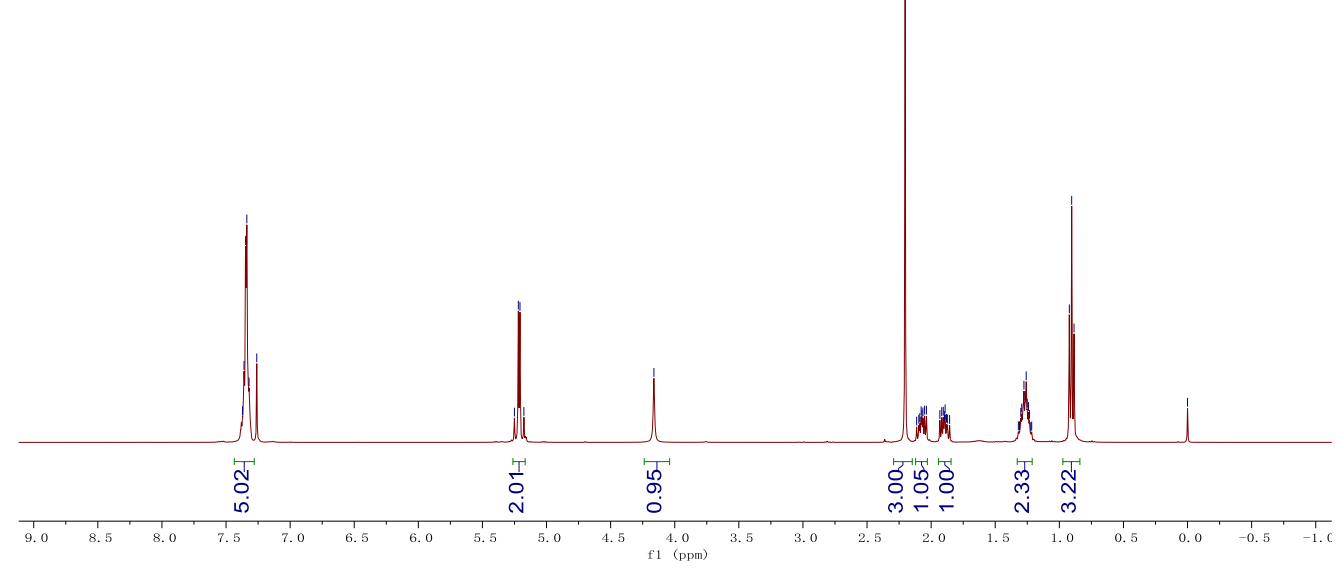

Figure S25. ${ }^{1} \mathrm{H}$ NMR $\left(400 \mathrm{MHz}, \mathrm{CDCl}_{3}\right)$ of $\mathbf{2 j}$.

\begin{tabular}{|c|c|c|c|}
\hline $\begin{array}{l}\infty \\
\infty \\
\infty \\
0 \\
1 \\
1\end{array}$ & 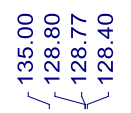 & 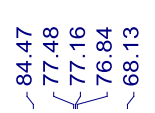 & $\stackrel{\substack{\infty \\
m}}{p}$ \\
\hline
\end{tabular}

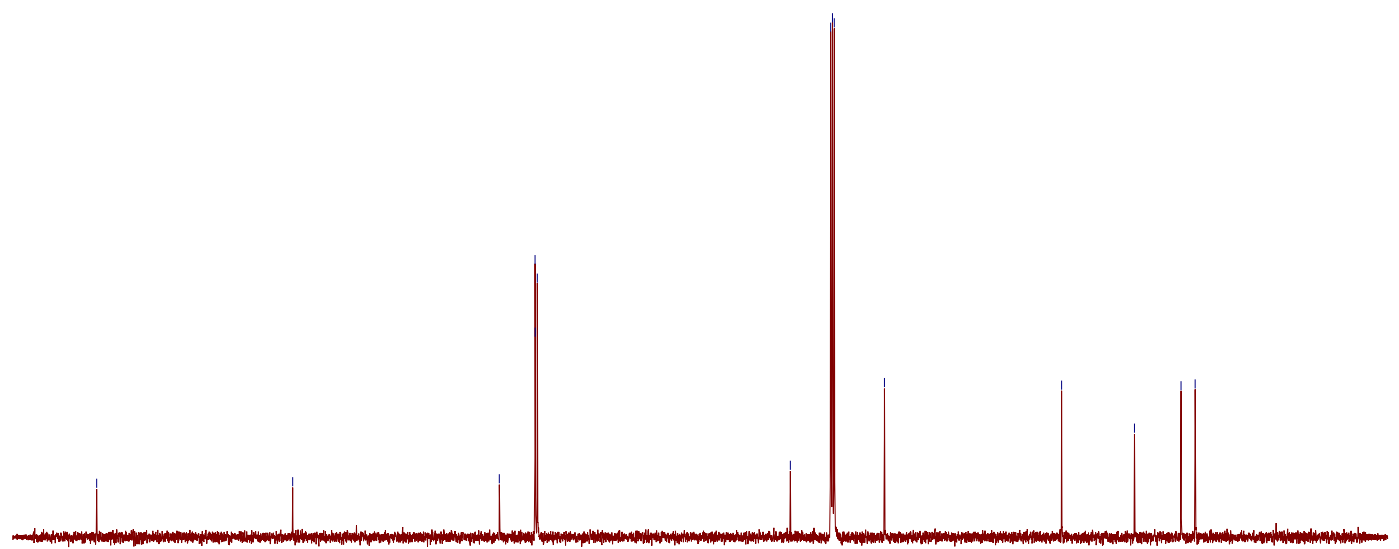

Figure S26. ${ }^{13} \mathrm{C}$ NMR $\left(100 \mathrm{MHz}, \mathrm{CDCl}_{3}\right)$ of $\mathbf{2 j}$. 


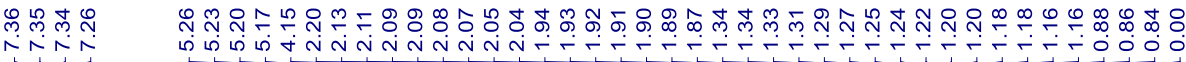
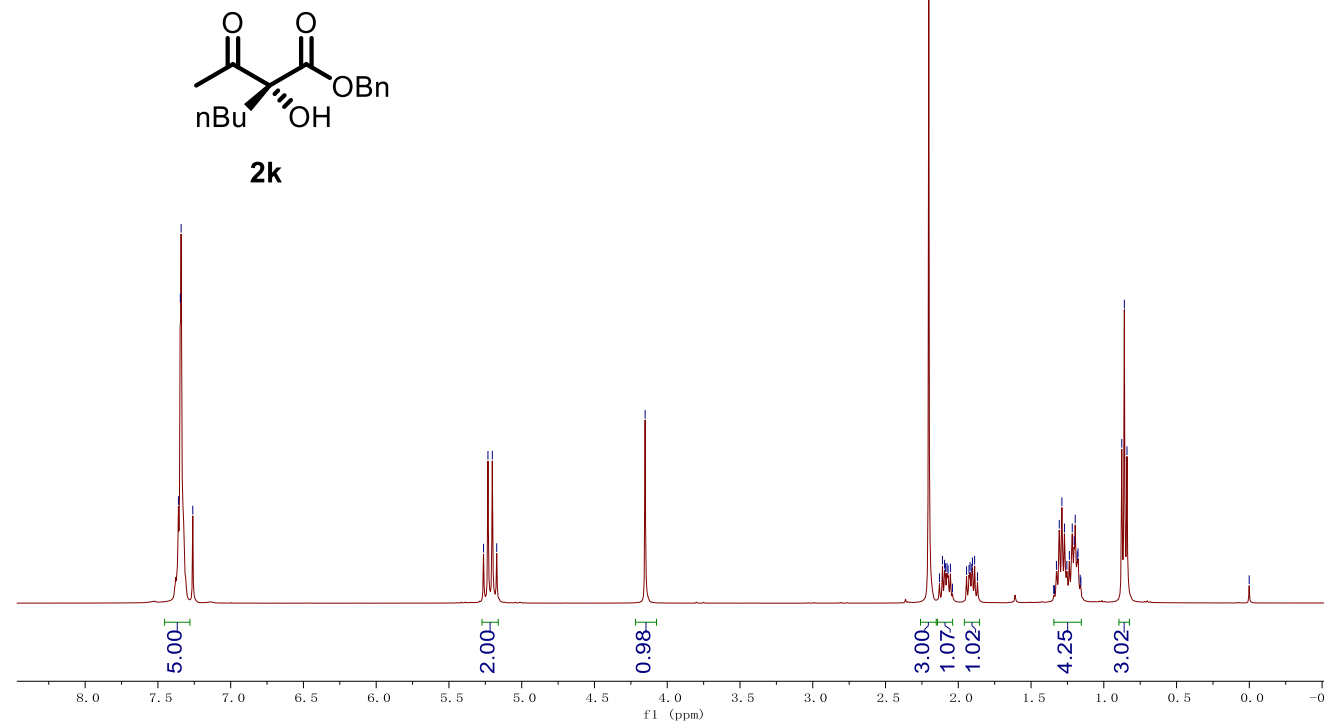

Figure S27. ${ }^{1} \mathrm{H}$ NMR $\left(400 \mathrm{MHz}, \mathrm{CDCl}_{3}\right)$ of $\mathbf{2 k}$.

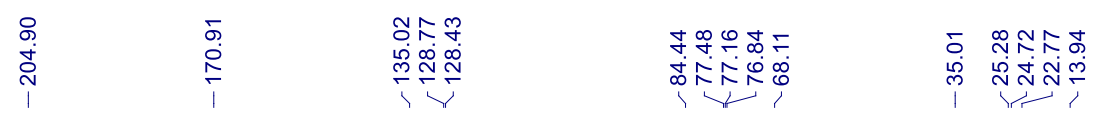
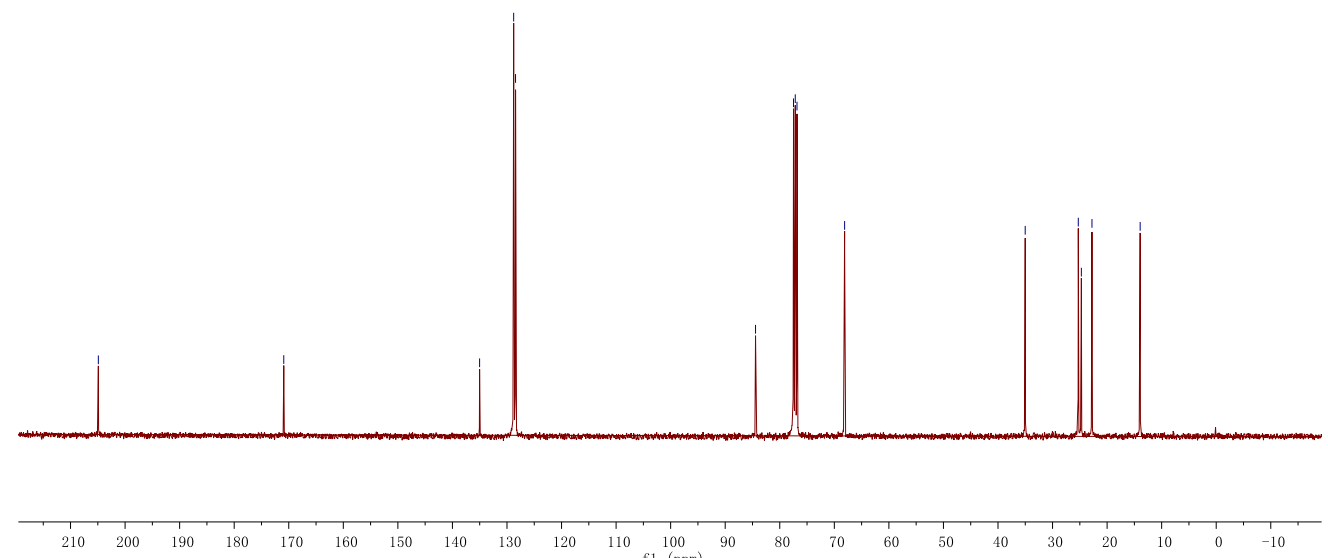

Figure S28. ${ }^{13} \mathrm{C}$ NMR $\left(100 \mathrm{MHz}, \mathrm{CDCl}_{3}\right)$ of $\mathbf{2 k}$. 


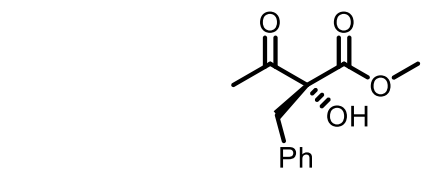

2)

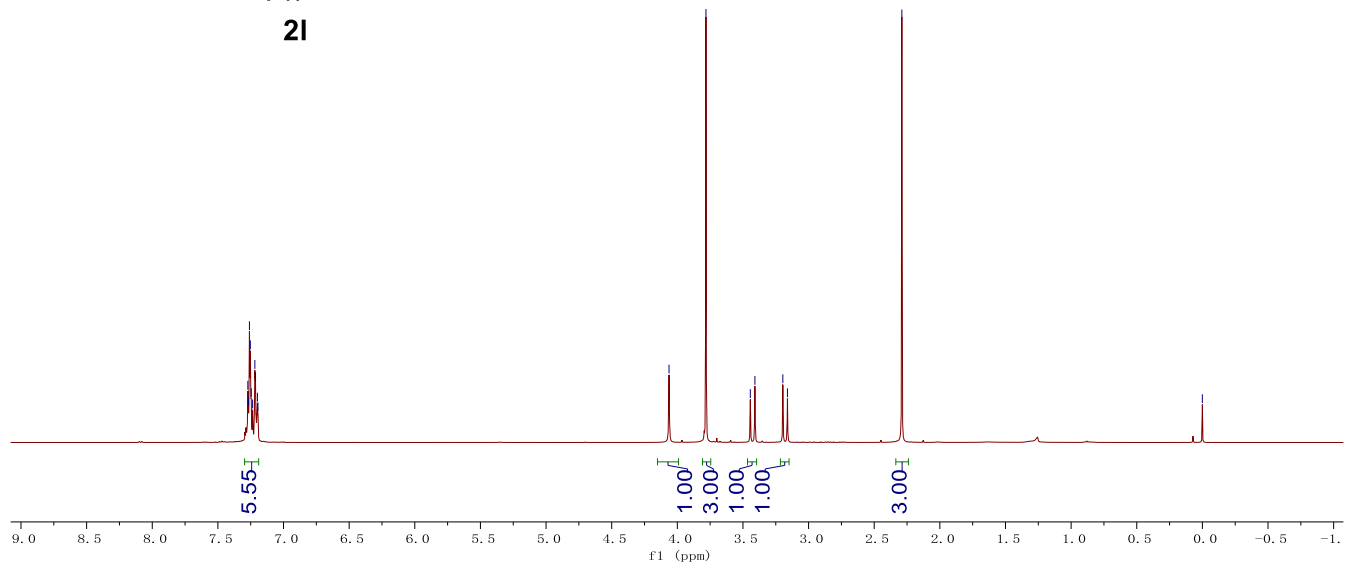

Figure S29. ${ }^{1} \mathrm{H}$ NMR $\left(400 \mathrm{MHz}, \mathrm{CDCl}_{3}\right)$ of 21.

\begin{tabular}{|c|c|c|c|c|}
\hline$\underset{1}{\stackrel{8}{\circ}}$ & matr & 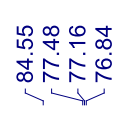 & $\begin{array}{l}\hat{L} \\
0 \\
0 \\
0 \\
1\end{array}$ & $\stackrel{\stackrel{0}{+}}{\dot{+}}$ \\
\hline
\end{tabular}
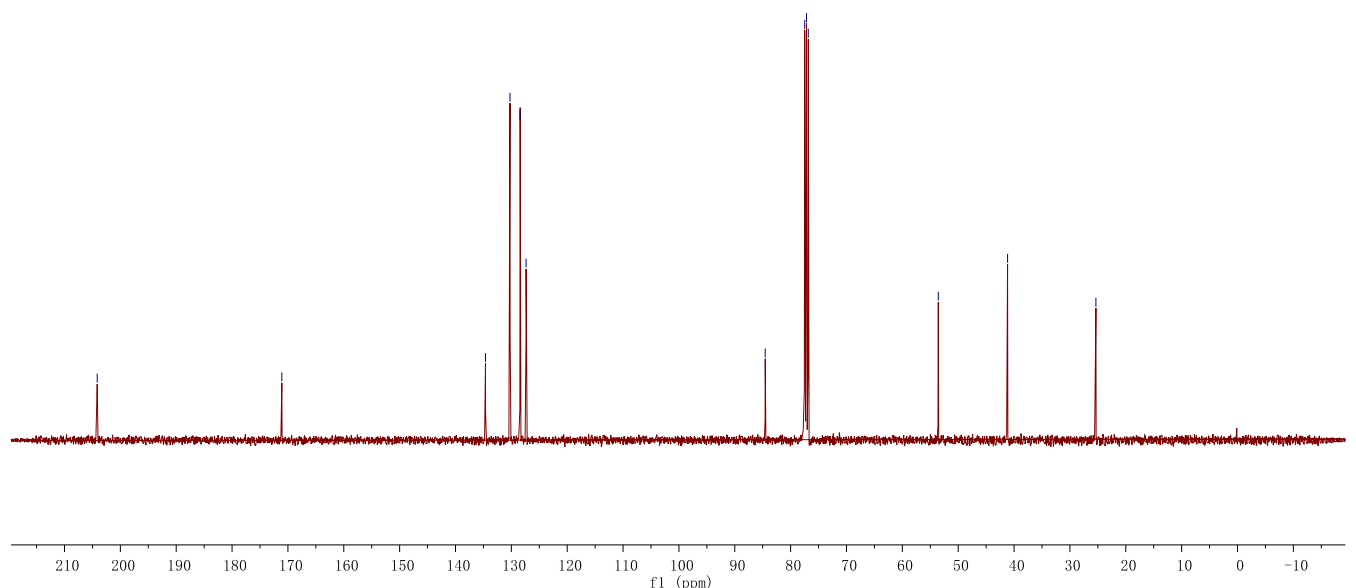

Figure S30. ${ }^{13} \mathrm{C}$ NMR $\left(100 \mathrm{MHz}, \mathrm{CDCl}_{3}\right)$ of $\mathbf{2 l}$. 

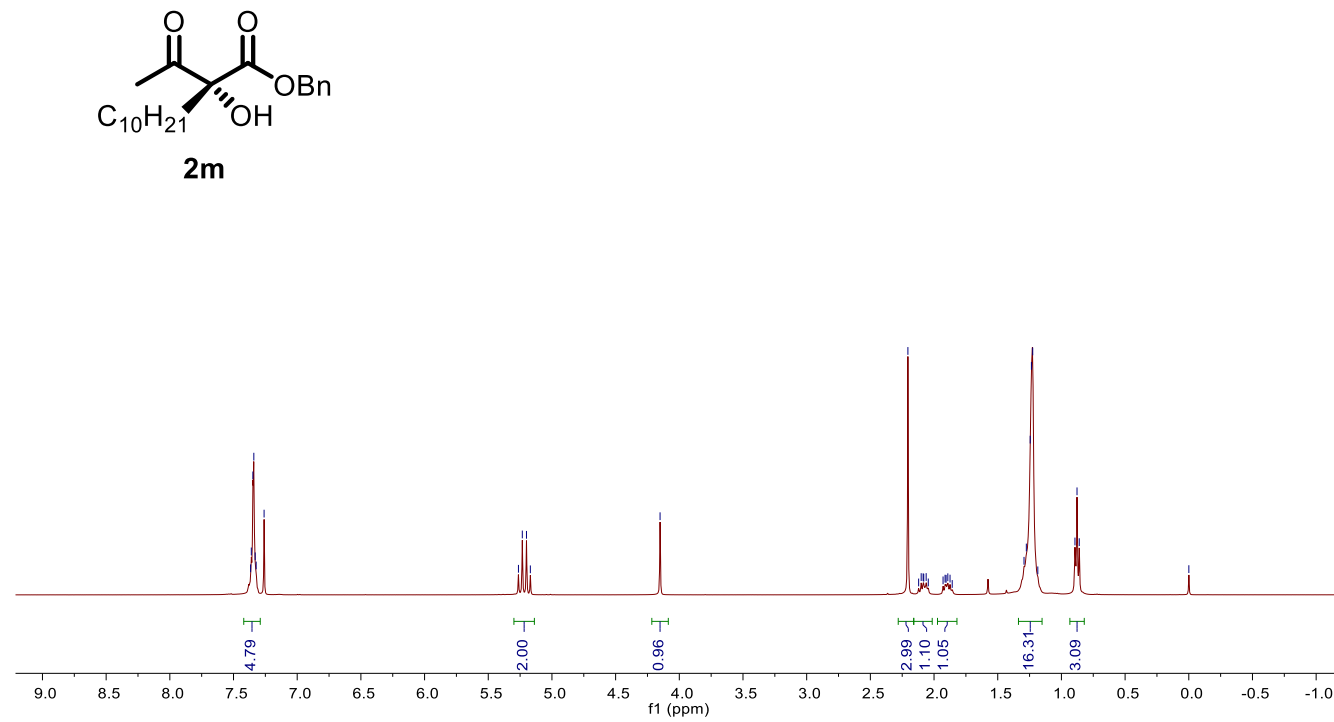

Figure S31. ${ }^{1} \mathrm{H}$ NMR $\left(400 \mathrm{MHz}, \mathrm{CDCl}_{3}\right)$ of $\mathbf{2 m}$.
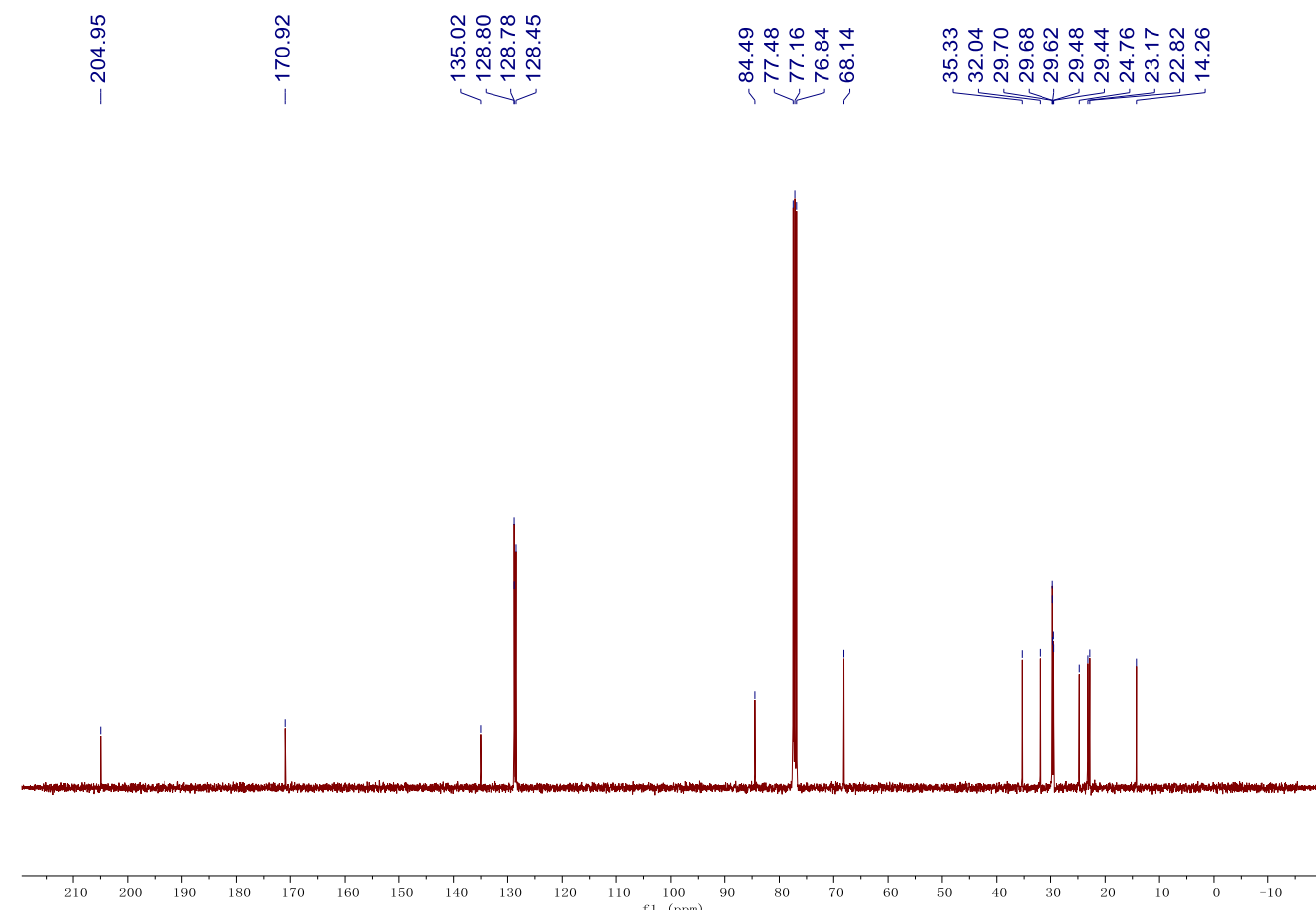

Figure S32. ${ }^{13} \mathrm{C}$ NMR $\left(100 \mathrm{MHz}, \mathrm{CDCl}_{3}\right)$ of $\mathbf{2 m}$. 


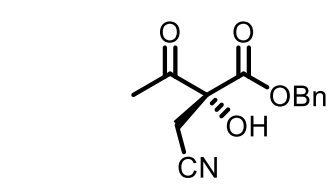

2n

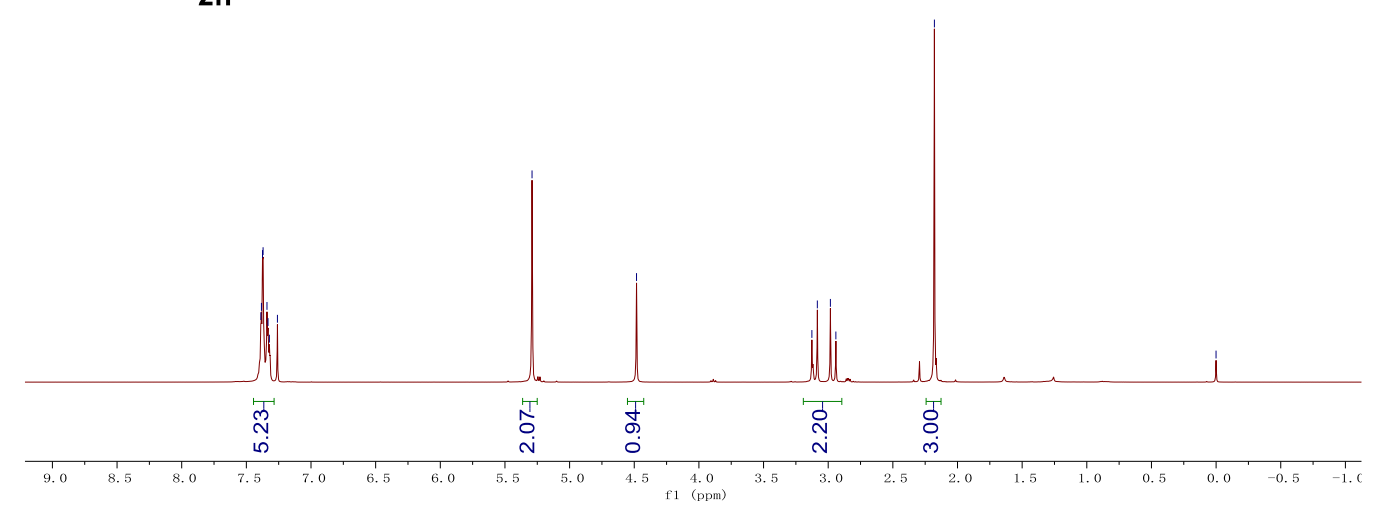

Figure S33. ${ }^{1} \mathrm{H}$ NMR $\left(400 \mathrm{MHz}, \mathrm{CDCl}_{3}\right)$ of $\mathbf{2 n}$.
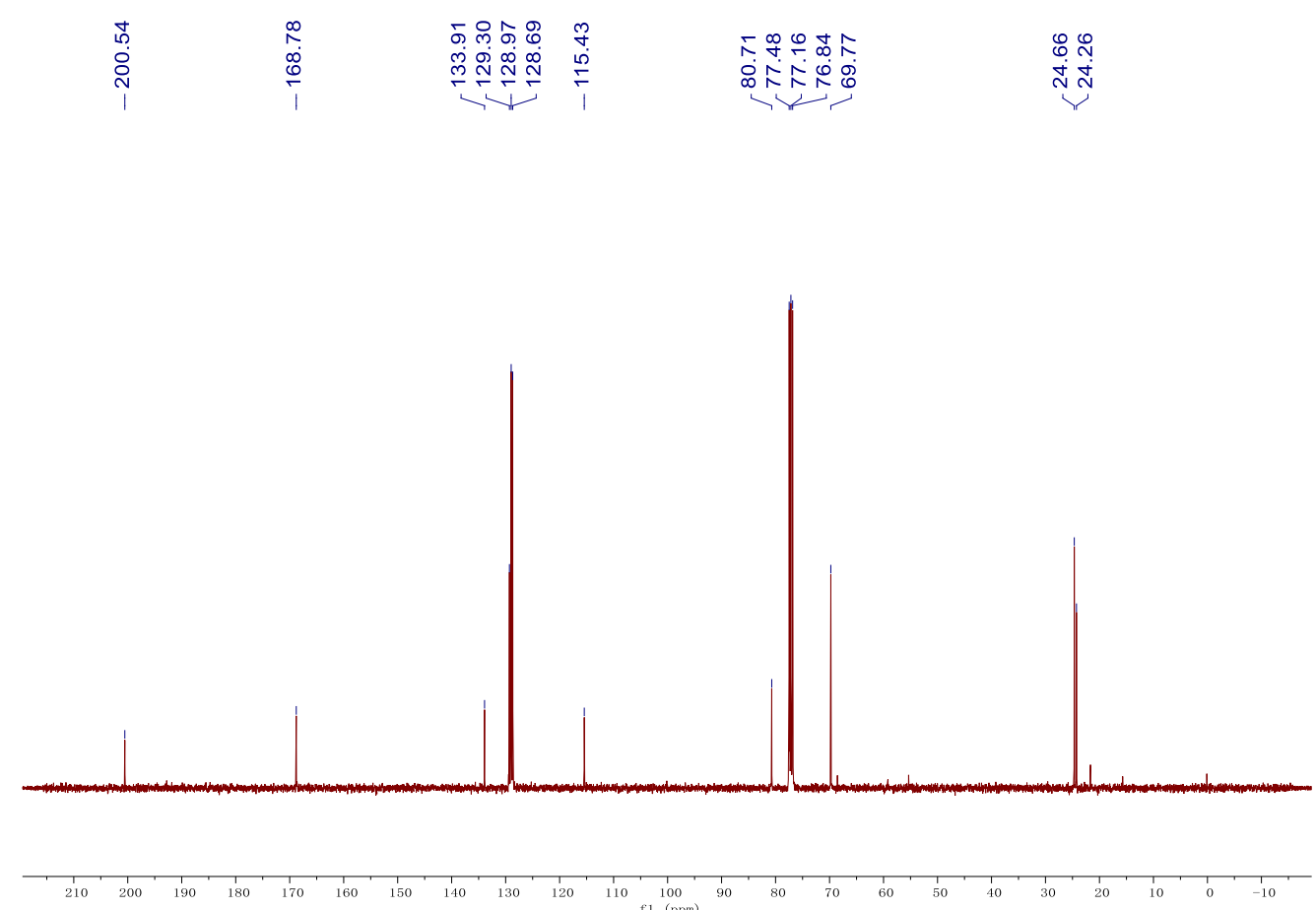

Figure S34. ${ }^{13} \mathrm{C}$ NMR $\left(100 \mathrm{MHz}, \mathrm{CDCl}_{3}\right)$ of $\mathbf{2 n}$. 


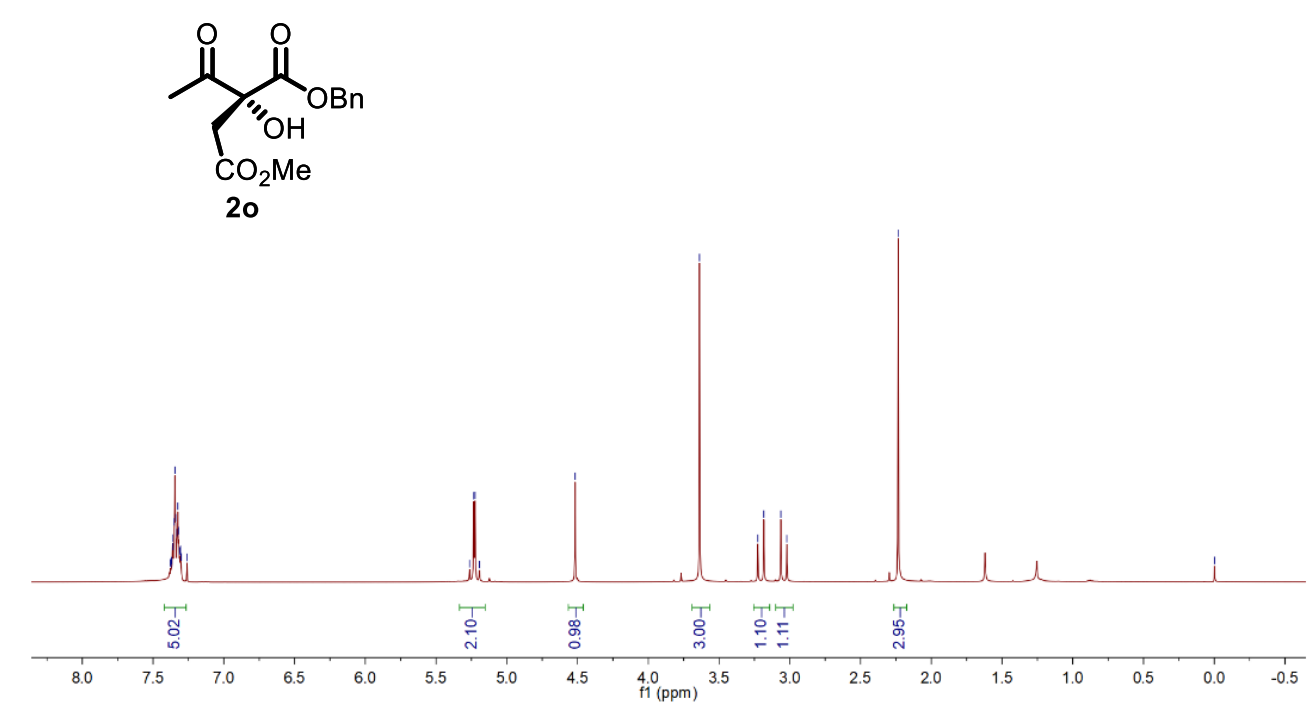

Figure S35. ${ }^{1} \mathrm{H}$ NMR $\left(400 \mathrm{MHz}, \mathrm{CDCl}_{3}\right)$ of $2 \mathbf{o}$.

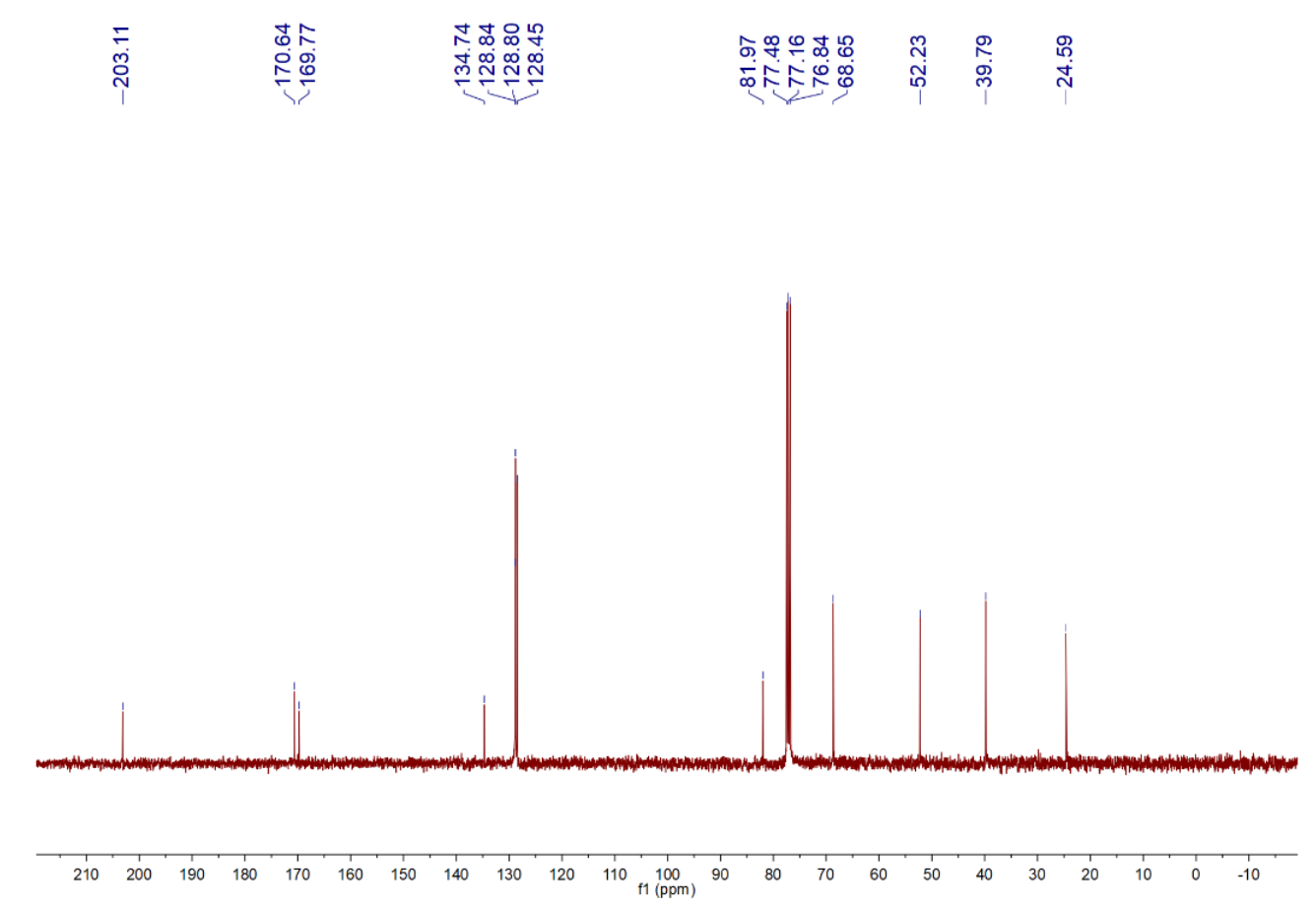

Figure S36. ${ }^{13} \mathrm{C}$ NMR $\left(100 \mathrm{MHz}, \mathrm{CDCl}_{3}\right)$ of $2 \mathrm{o}$. 


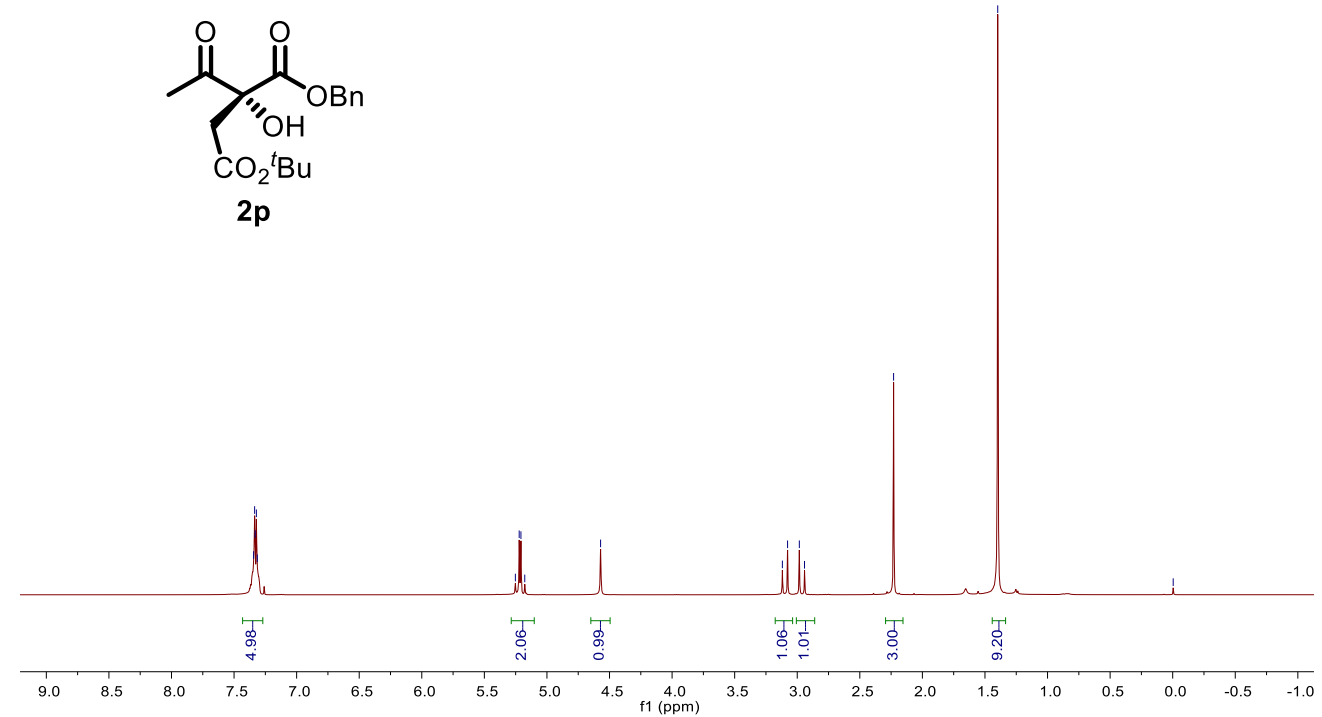

Figure S37. ${ }^{1} \mathrm{H} \mathrm{NMR}\left(400 \mathrm{MHz}, \mathrm{CDCl}_{3}\right)$ of $\mathbf{2 p}$.

\begin{tabular}{|c|c|c|c|}
\hline $\begin{array}{l}\infty \\
\infty \\
\infty \\
0 \\
0.0 \\
0.0 \\
0\end{array}$ & 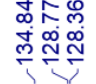 & 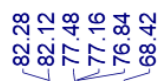 & $\frac{\dot{y}}{\dot{q}}$ \\
\hline
\end{tabular}

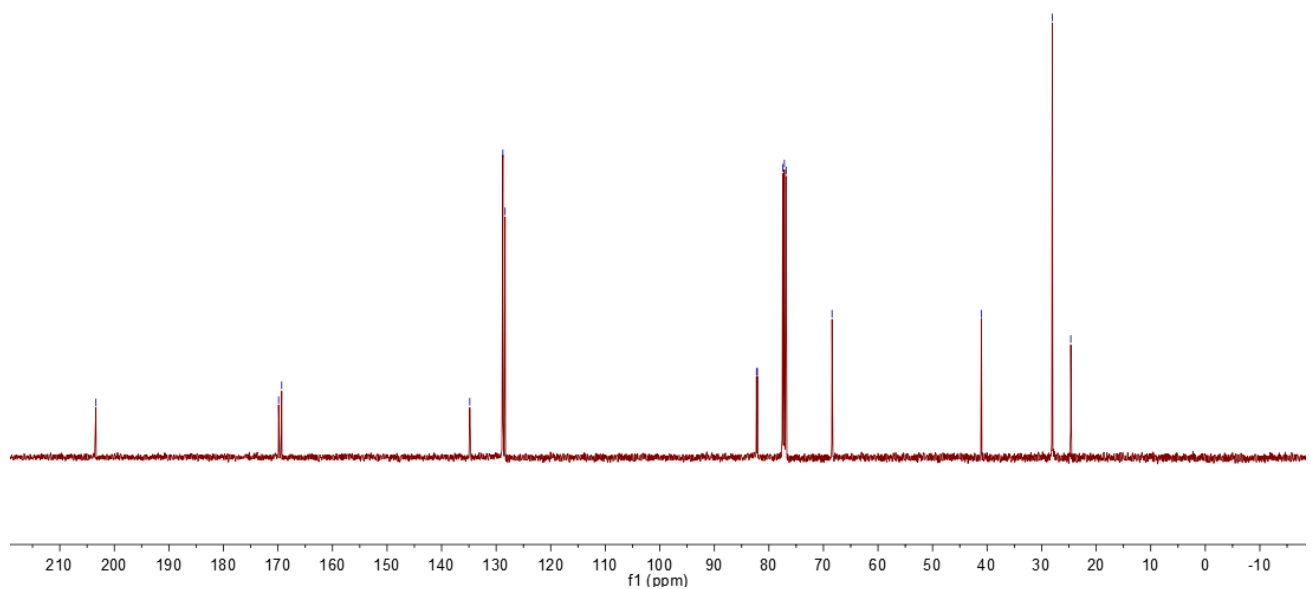

Figure S38. ${ }^{13} \mathrm{C}$ NMR $\left(100 \mathrm{MHz}, \mathrm{CDCl}_{3}\right)$ of $\mathbf{2 p}$. 


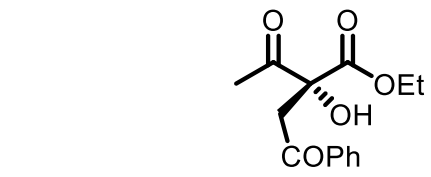

2q

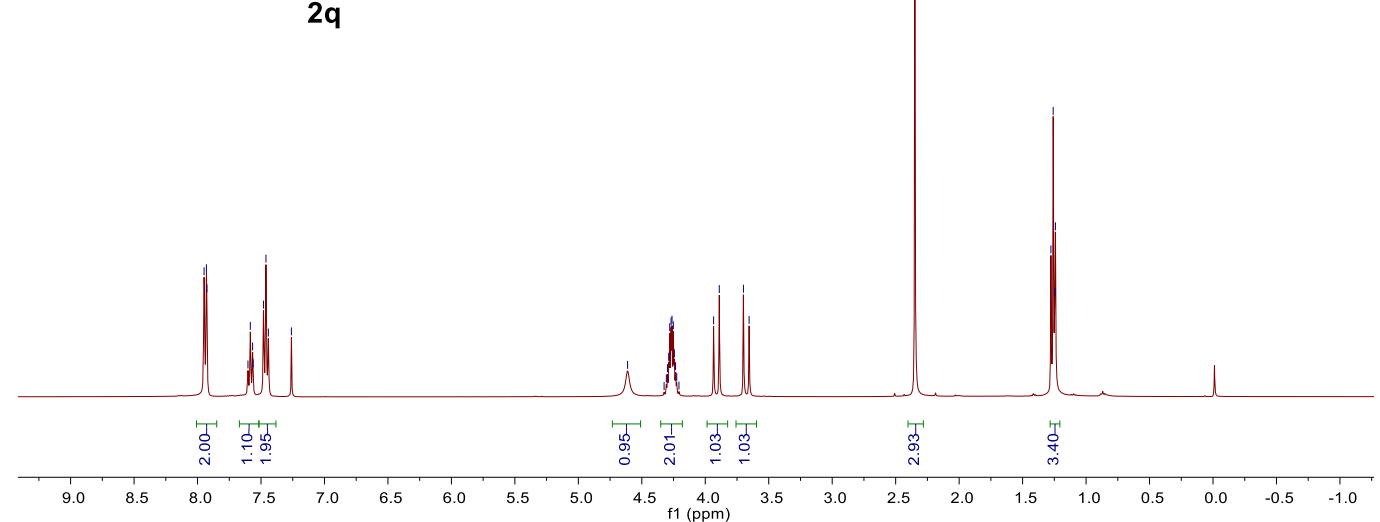

Figure S39. ${ }^{1} \mathrm{H}$ NMR $\left(400 \mathrm{MHz}, \mathrm{CDCl}_{3}\right)$ of $\mathbf{2 q}$.

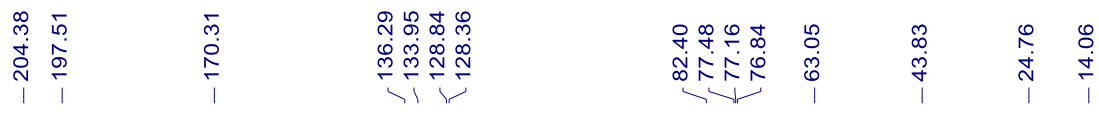

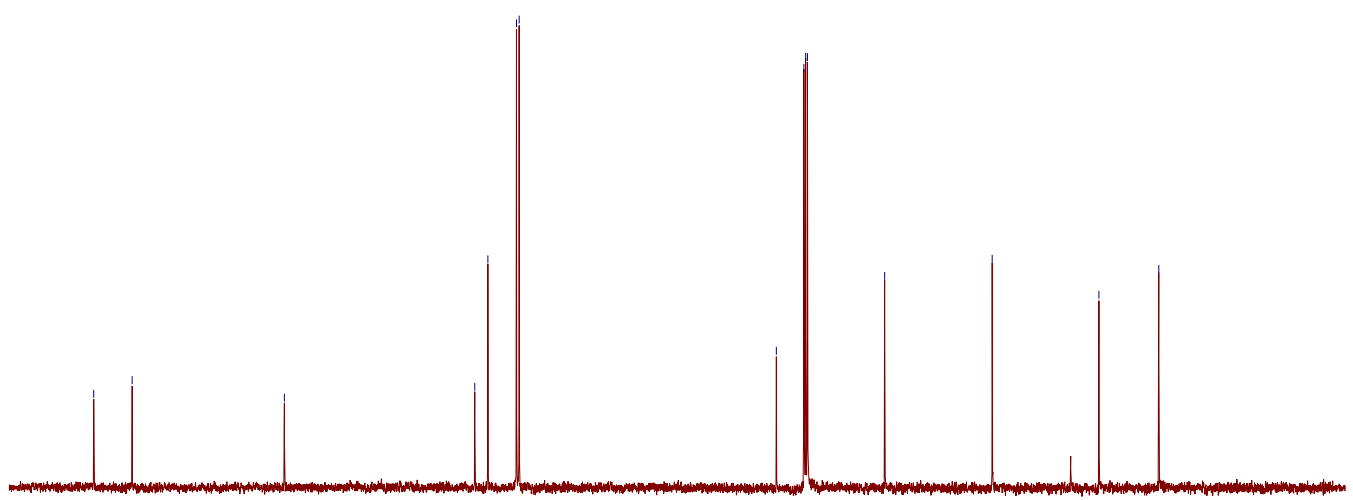

$\begin{array}{lllllllllllll}210 & 200 & 190 & 180 & 170 & 160 & 150 & 140 & 130 & 120 & 110 & 100 & 90\end{array}$

Figure S40. ${ }^{13} \mathrm{C}$ NMR $\left(100 \mathrm{MHz}, \mathrm{CDCl}_{3}\right)$ of $\mathbf{2 q}$. 
<smiles>CCOC(=O)C(O)(CC1OCCO1)C(C)=O</smiles>

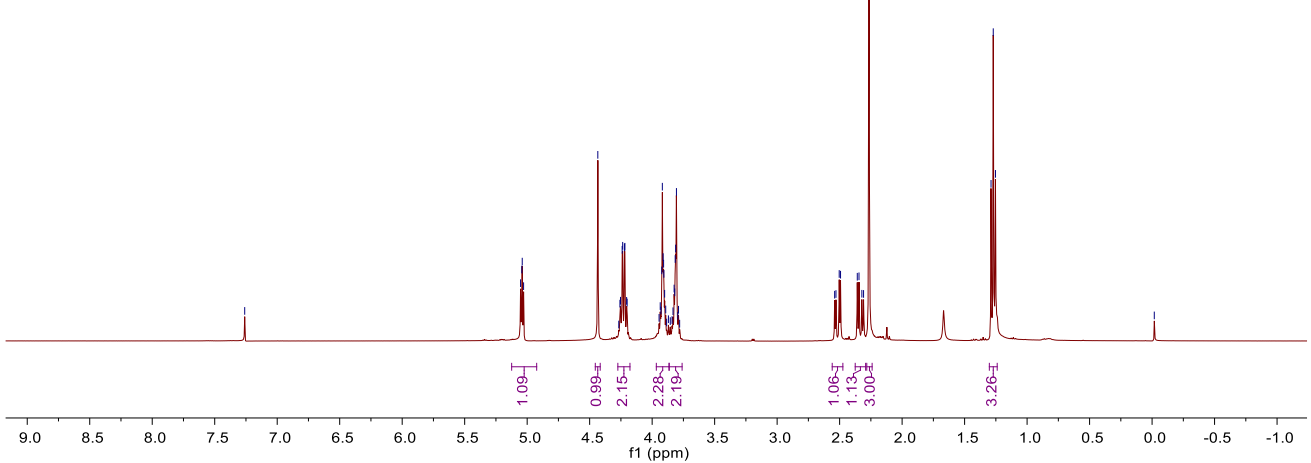

Figure S41. ${ }^{1} \mathrm{H}$ NMR $\left(400 \mathrm{MHz}, \mathrm{CDCl}_{3}\right)$ of $\mathbf{2 r}$.

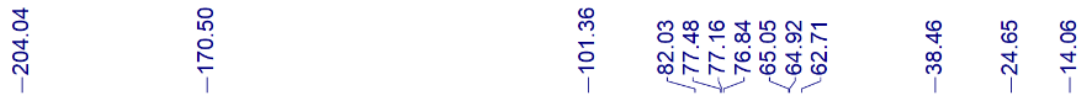

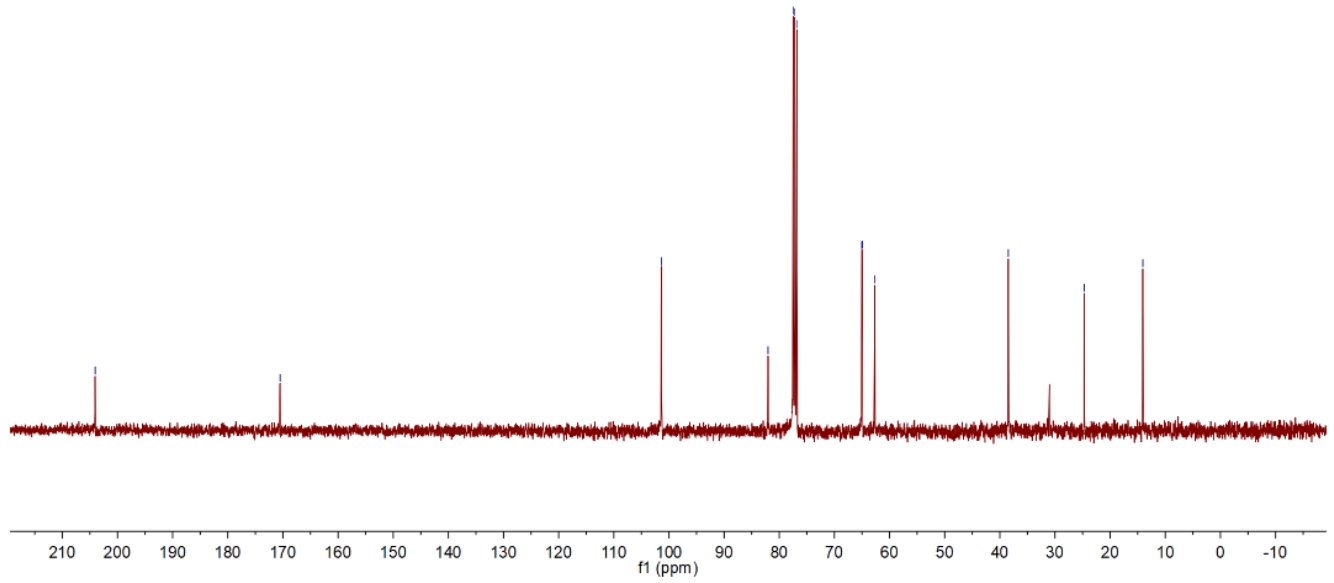

Figure $\mathbf{S 4 2} .{ }^{13} \mathrm{C}$ NMR $\left(100 \mathrm{MHz}, \mathrm{CDCl}_{3}\right)$ of $\mathbf{2 r}$. 


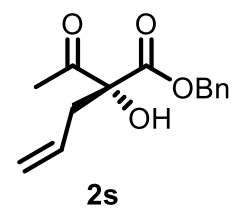

$2 \mathrm{~s}$

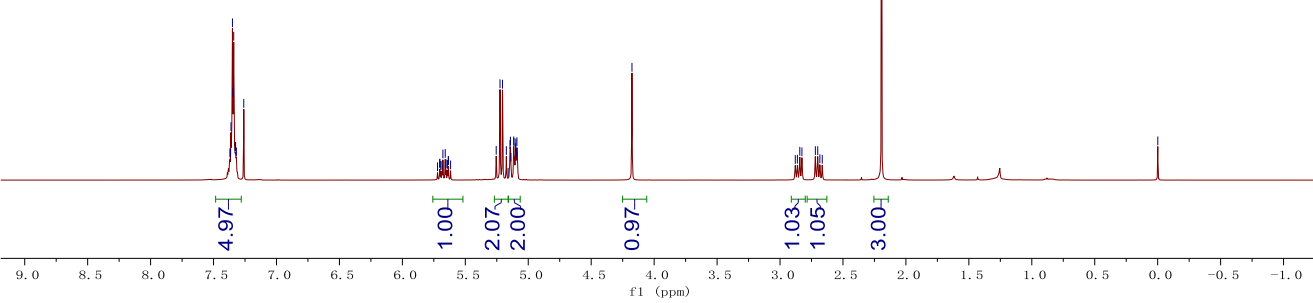

Figure $\mathbf{S 4 3} .{ }^{1} \mathrm{H}$ NMR $\left(400 \mathrm{MHz}, \mathrm{CDCl}_{3}\right)$ of $\mathbf{2 s}$.

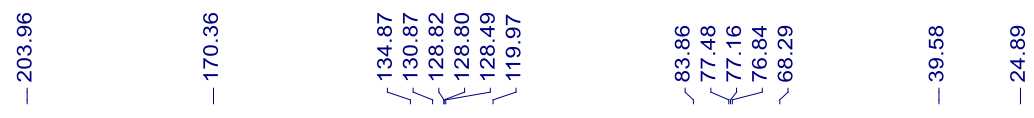

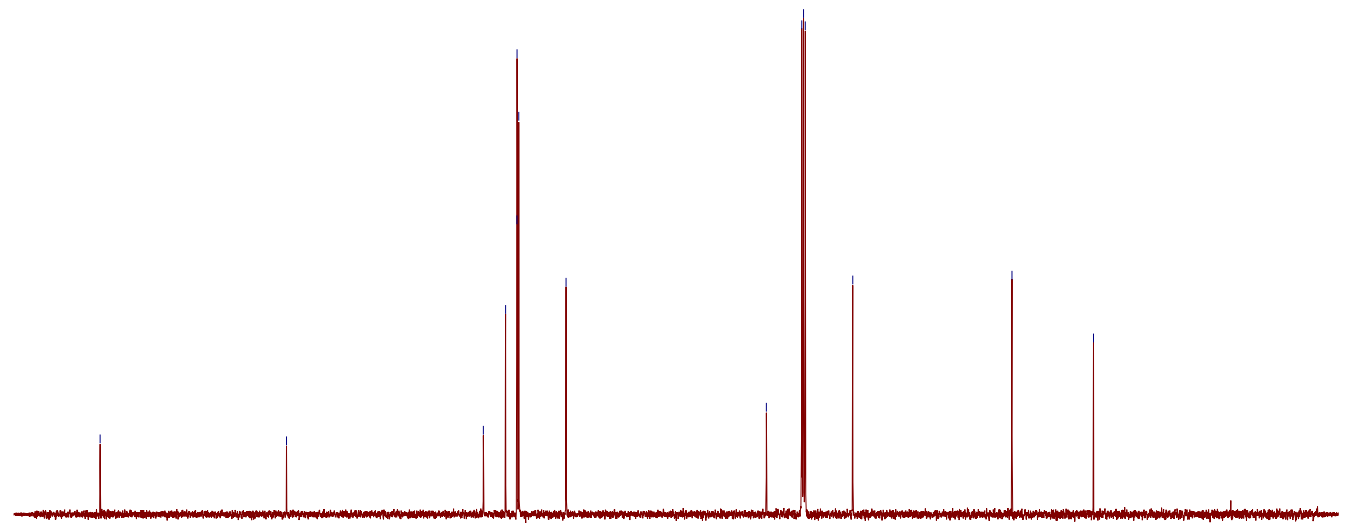

Figure S44. ${ }^{13} \mathrm{C}$ NMR $\left(100 \mathrm{MHz}, \mathrm{CDCl}_{3}\right)$ of $\mathbf{2 s .}$ 

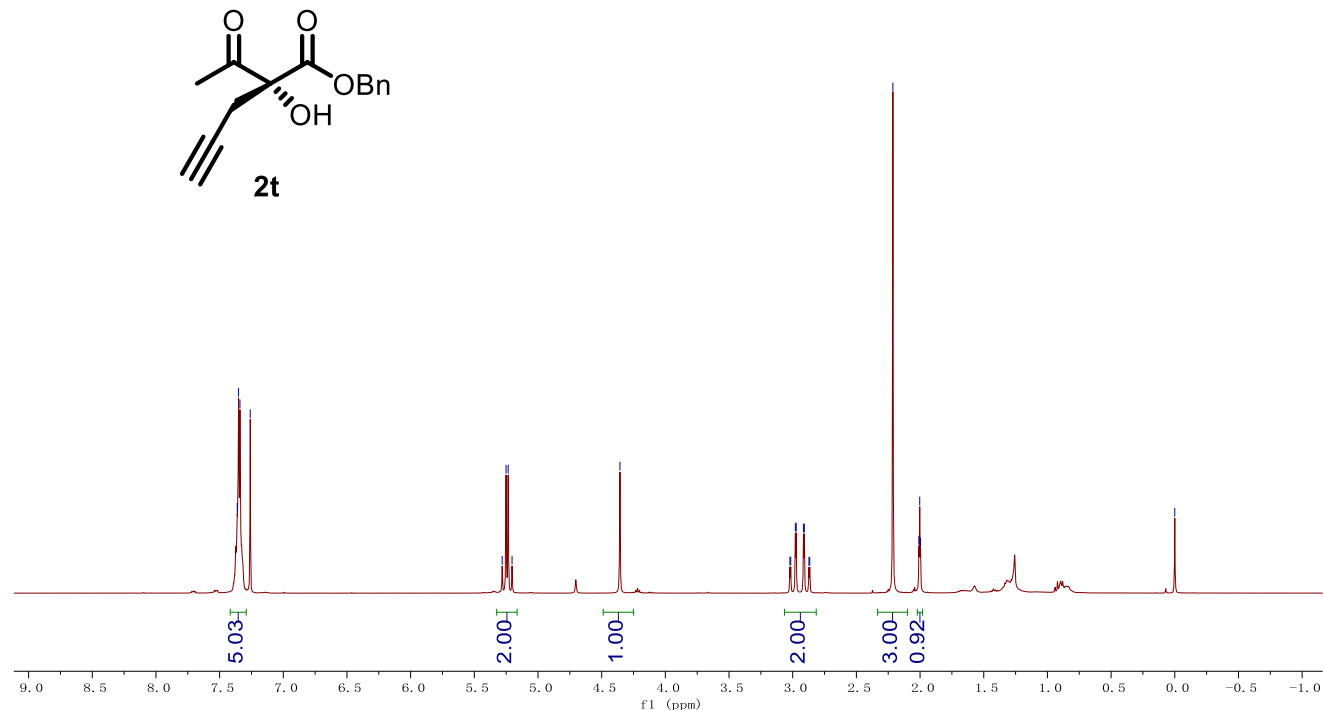

Figure $\mathbf{S 4 5} .{ }^{1} \mathrm{H}$ NMR $\left(400 \mathrm{MHz}, \mathrm{CDCl}_{3}\right)$ of $\mathbf{2 t}$.

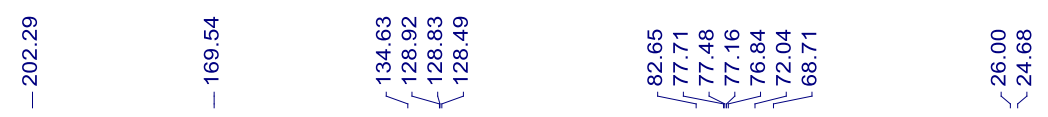
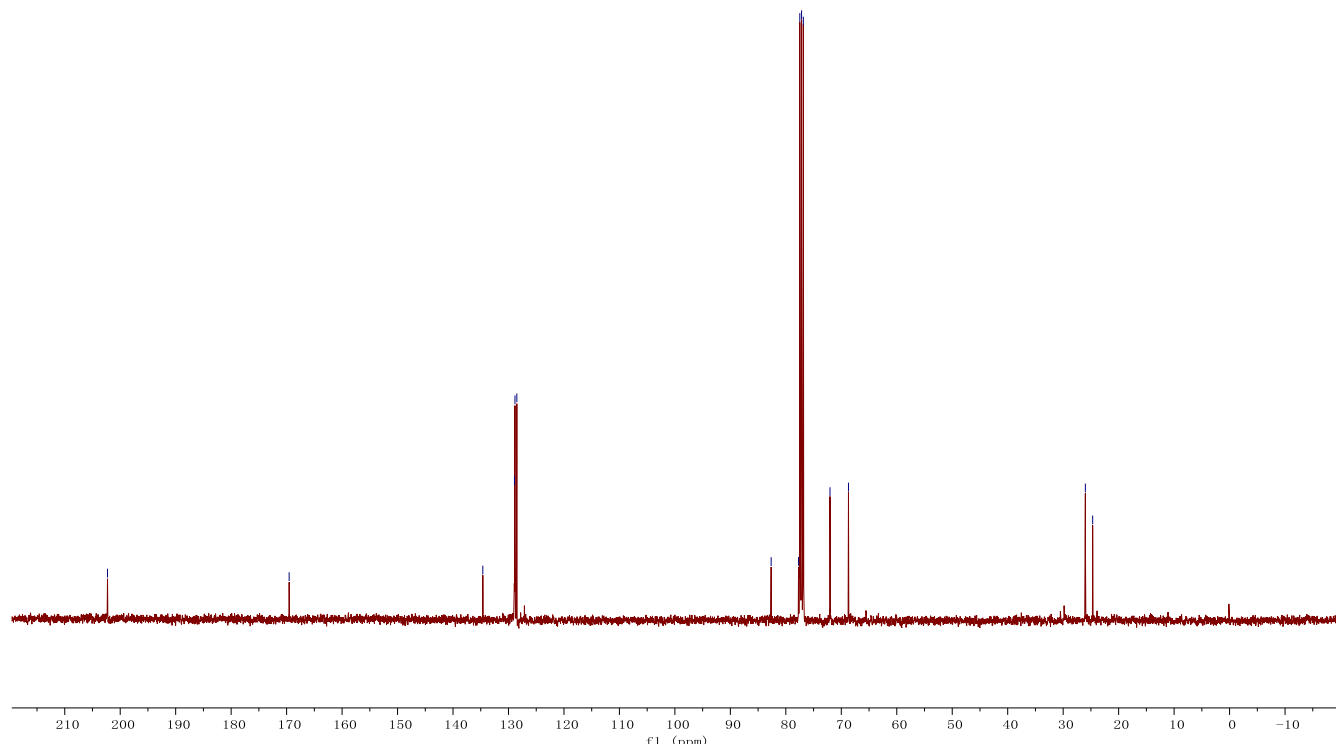

Figure S46. ${ }^{13} \mathrm{C}$ NMR $\left(100 \mathrm{MHz}, \mathrm{CDCl}_{3}\right)$ of $\mathbf{2 t}$. 


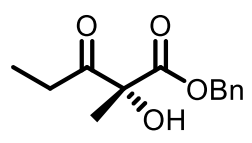

2u

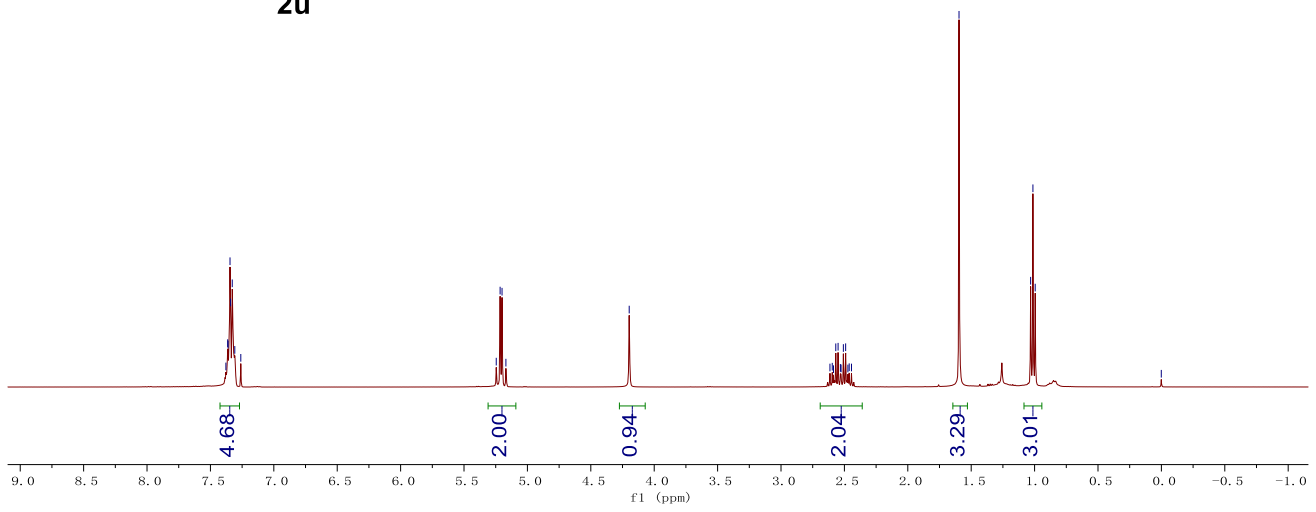

Figure S47. ${ }^{1} \mathrm{H}$ NMR $\left(400 \mathrm{MHz}, \mathrm{CDCl}_{3}\right)$ of $\mathbf{2 u}$.

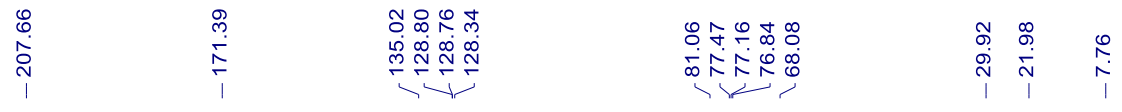

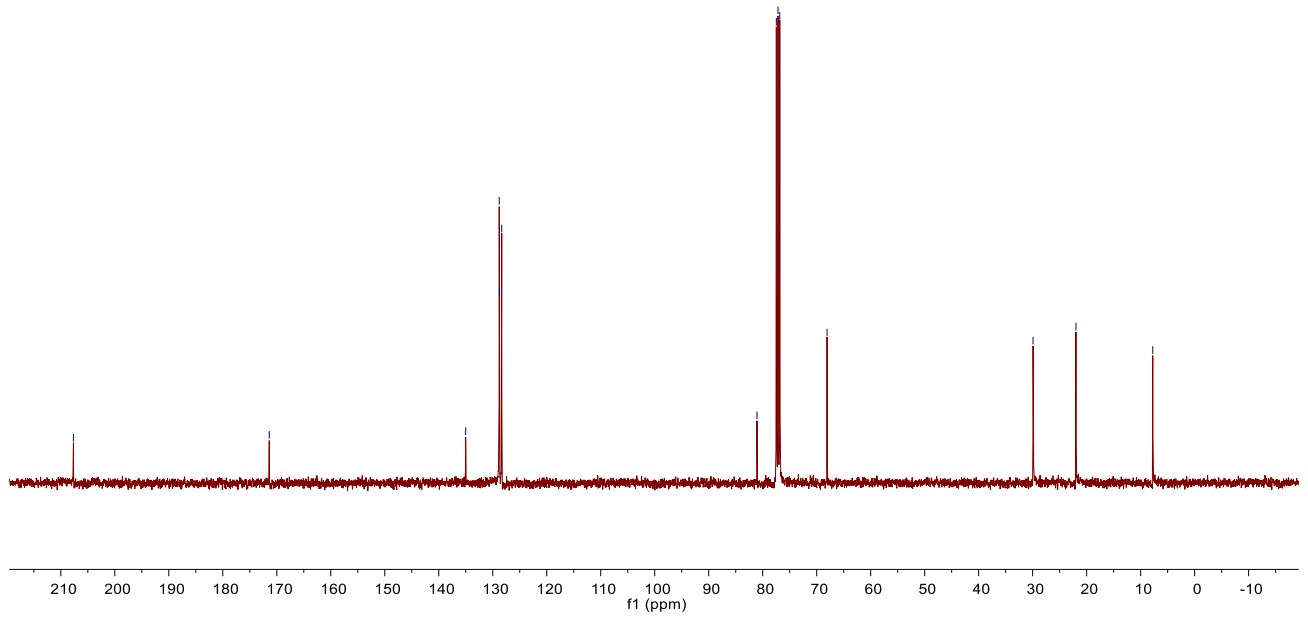

Figure S48. ${ }^{13} \mathrm{C}$ NMR $\left(100 \mathrm{MHz}, \mathrm{CDCl}_{3}\right)$ of $\mathbf{2 u}$. 

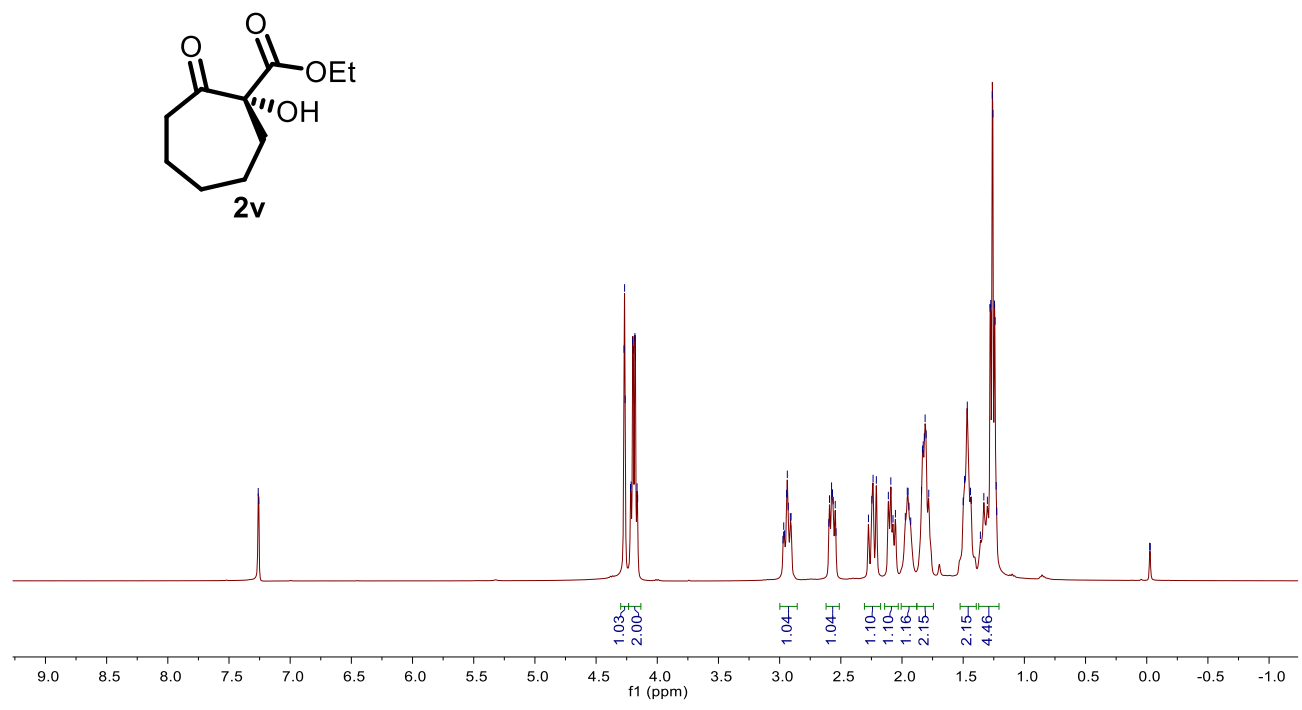

Figure S49. ${ }^{1} \mathrm{H}$ NMR $\left(400 \mathrm{MHz}, \mathrm{CDCl}_{3}\right)$ of $\mathbf{2 v}$.

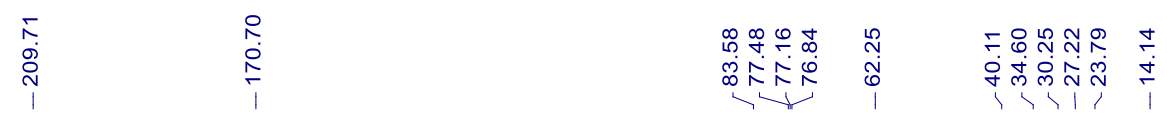

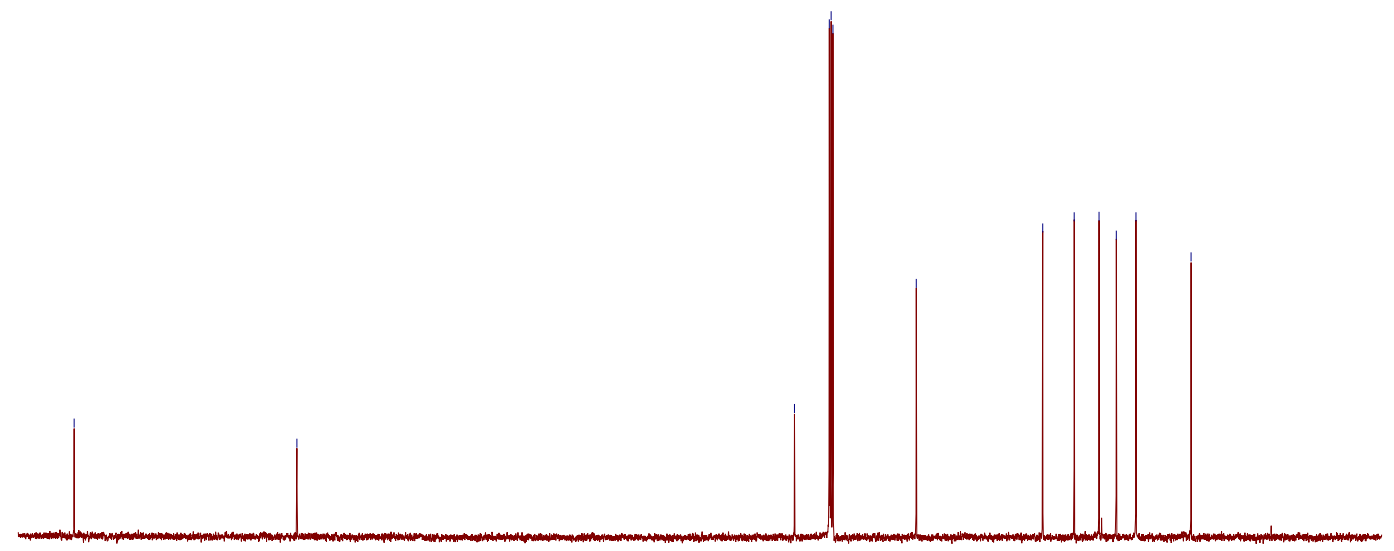

Figure S50. ${ }^{13} \mathrm{C}$ NMR $\left(100 \mathrm{MHz}, \mathrm{CDCl}_{3}\right)$ of $\mathbf{2 v}$. 

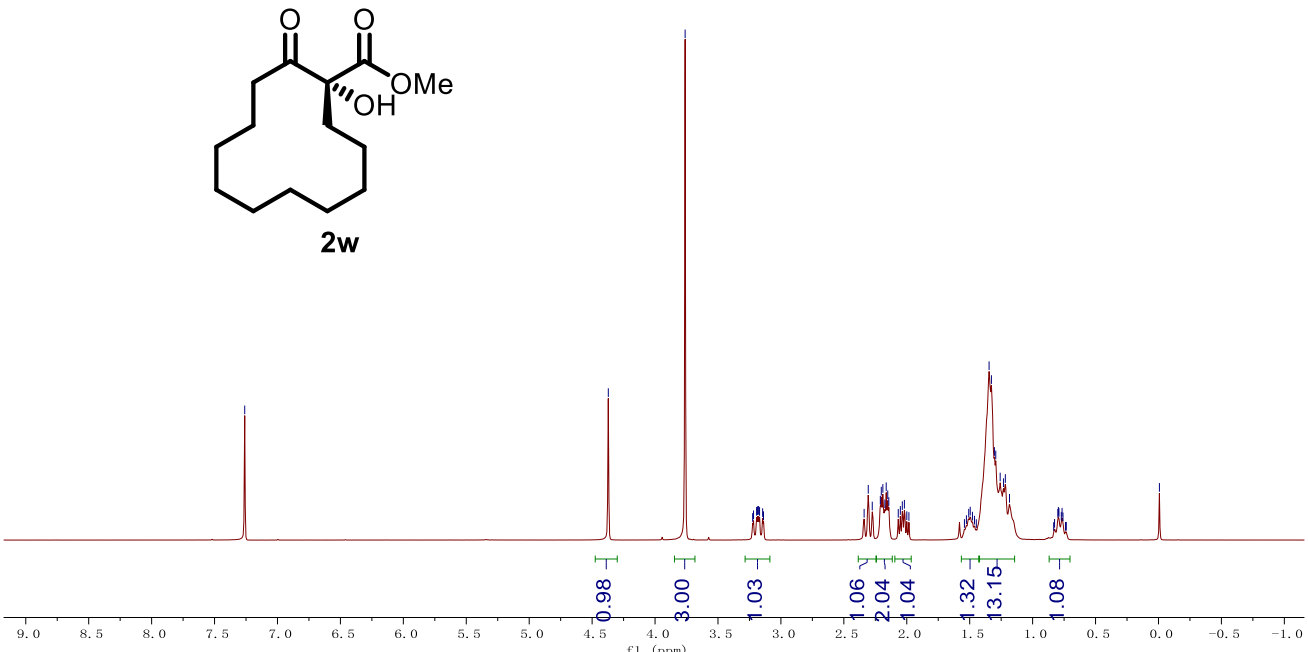

Figure S51. ${ }^{1} \mathrm{H}$ NMR (400 MHz, $\left.\mathrm{CDCl}_{3}\right)$ of $\mathbf{2 w}$.
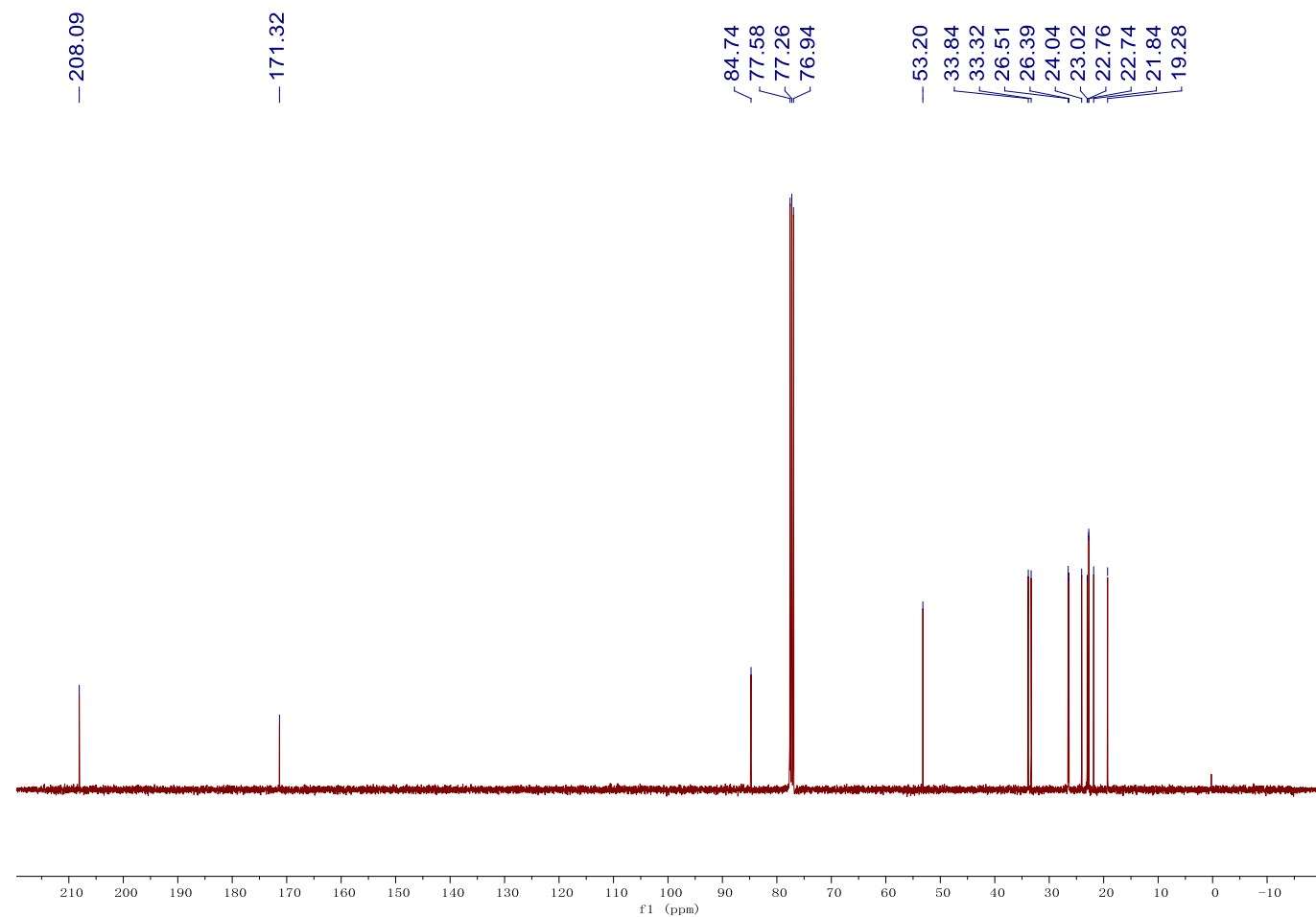

Figure S52. ${ }^{13} \mathrm{C}$ NMR $\left(100 \mathrm{MHz}, \mathrm{CDCl}_{3}\right)$ of $\mathbf{2 w}$. 


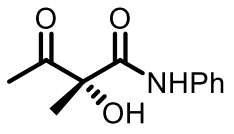

6a

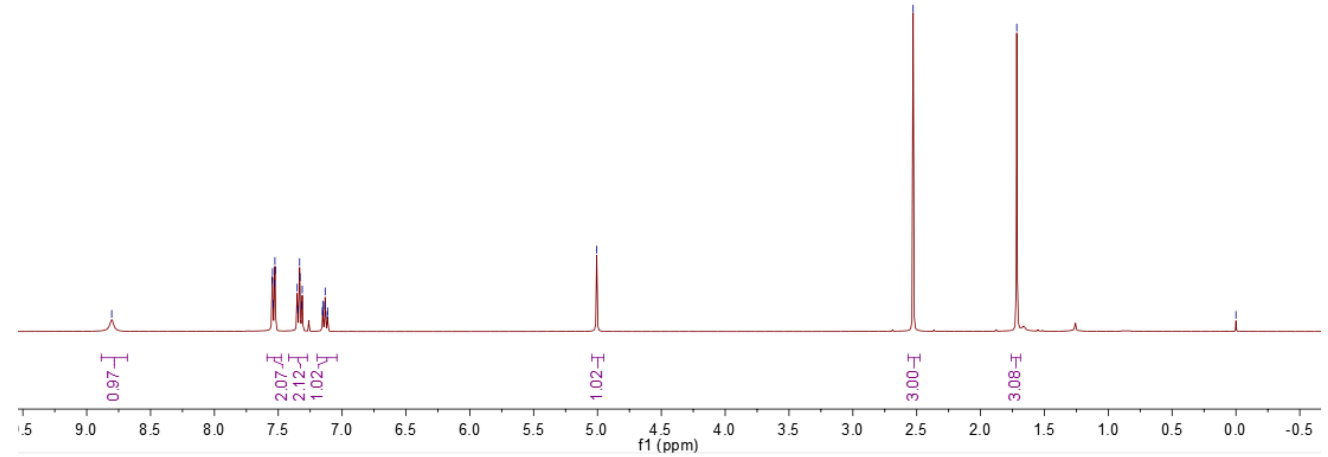

Figure S53. ${ }^{1} \mathrm{H}$ NMR $\left(400 \mathrm{MHz}, \mathrm{CDCl}_{3}\right)$ of $6 \mathbf{6}$.

\begin{tabular}{|c|c|c|}
\hline $\begin{array}{l}\text { 웅 } \\
\stackrel{0}{0} \\
\stackrel{0}{1}\end{array}$ & 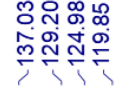 & $\underset{\infty}{\stackrel{N}{N}}$ \\
\hline
\end{tabular}

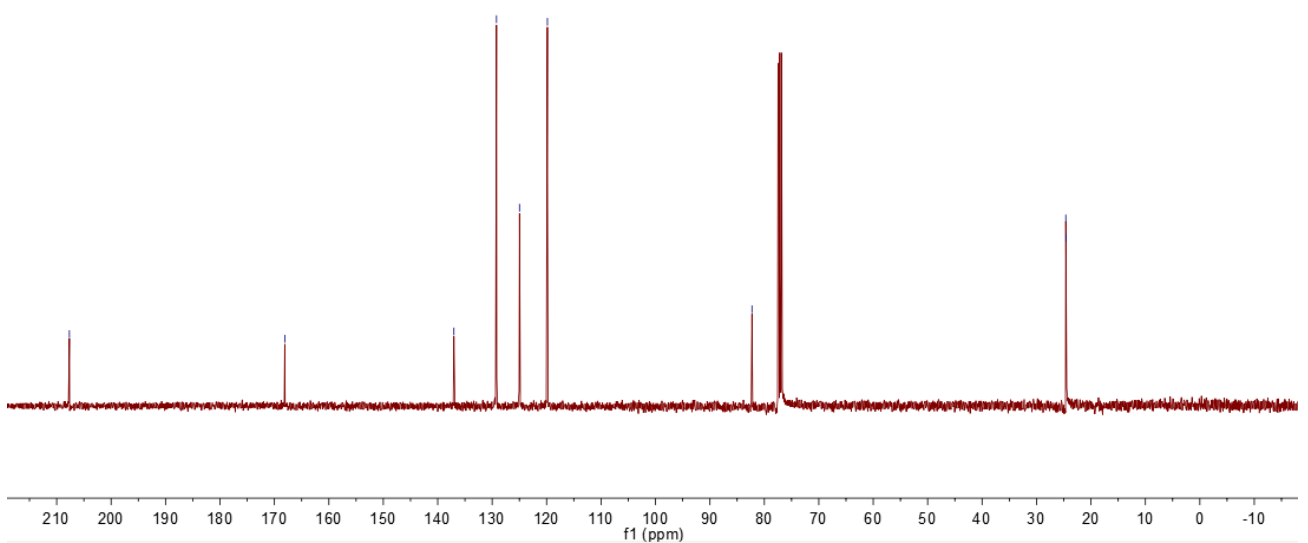

Figure S54. ${ }^{13} \mathrm{C}$ NMR $\left(100 \mathrm{MHz}, \mathrm{CDCl}_{3}\right)$ of $\mathbf{6 a}$. 


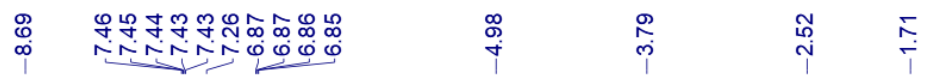<smiles>COc1ccc(NC(=O)[C@](C)(O)C(C)=O)cc1</smiles>

6b

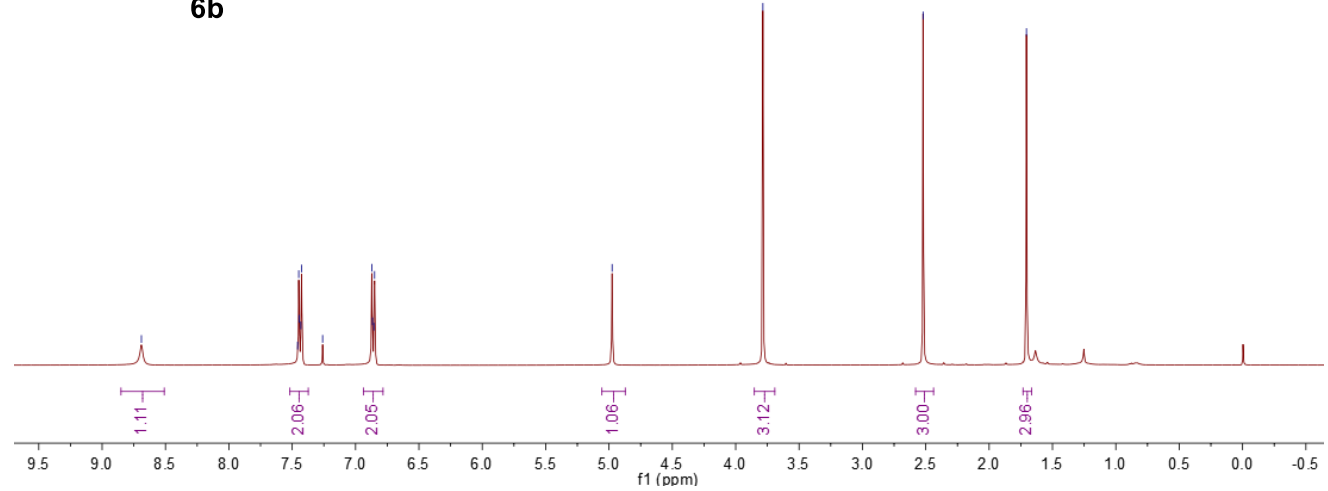

Figure S55. ${ }^{1} \mathrm{H}$ NMR (400 MHz, $\left.\mathrm{CDCl}_{3}\right)$ of $\mathbf{6 b}$.

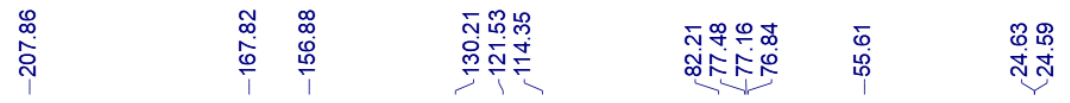

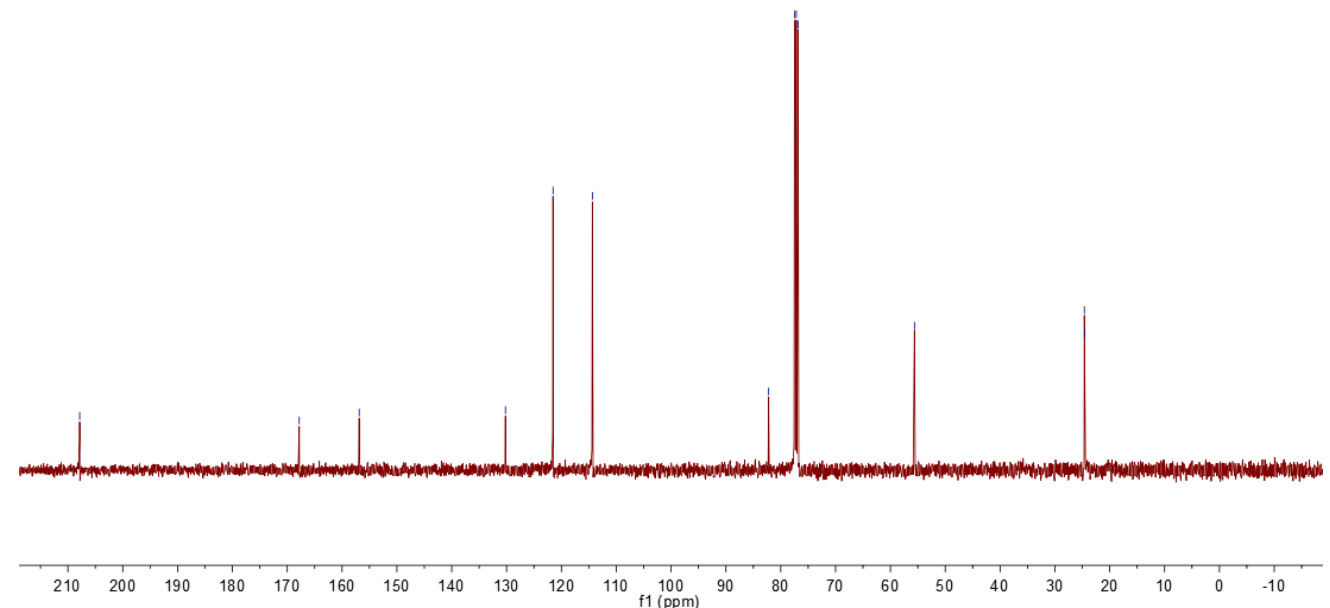

Figure S56. ${ }^{13} \mathrm{C} \mathrm{NMR}\left(100 \mathrm{MHz}, \mathrm{CDCl}_{3}\right)$ of $\mathbf{6 b}$. 


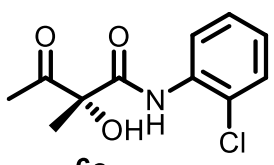

6c

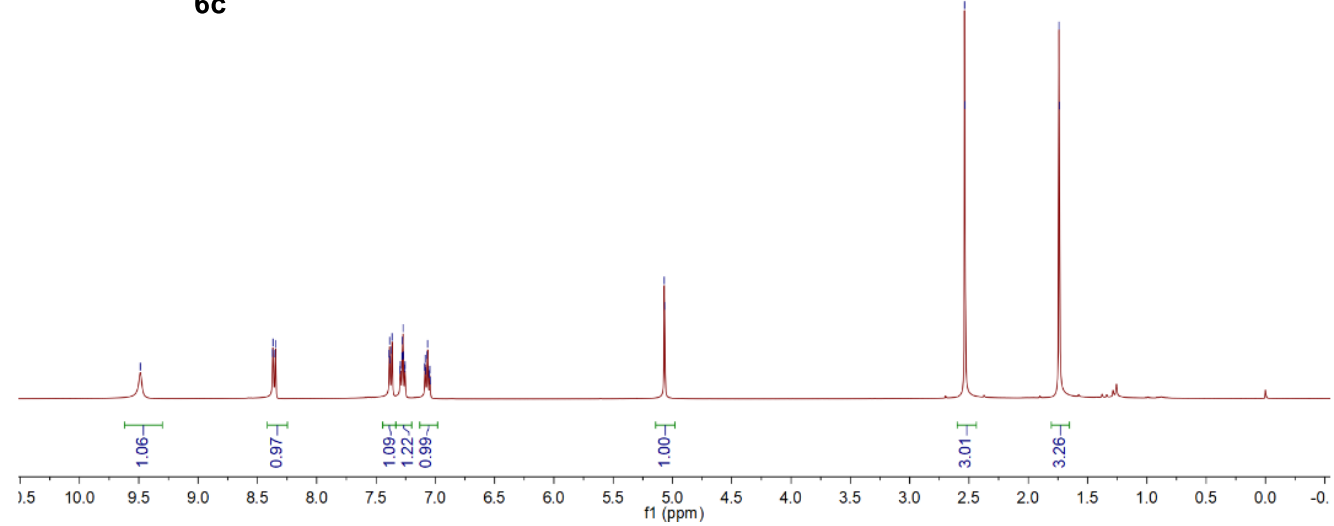

Figure S57. ${ }^{1} \mathrm{H}$ NMR $\left(400 \mathrm{MHz}, \mathrm{CDCl}_{3}\right)$ of $\mathbf{6 c}$.

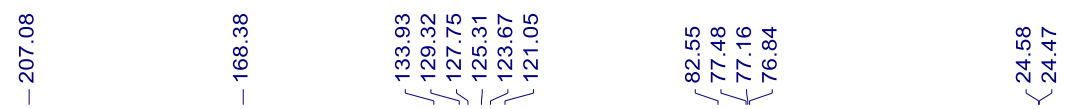

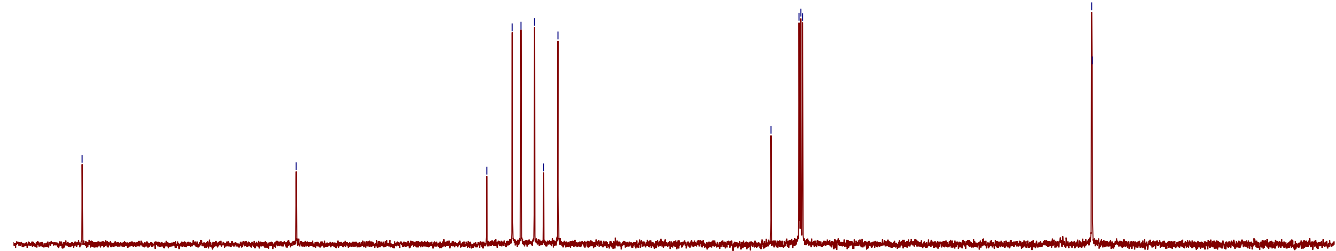

$\begin{array}{lllllllllllllllllllllll}210 & 200 & 190 & 180 & 170 & 160 & 150 & 140 & 130 & 120 & 110 & 100 & 90 & 80 & 70 & 60 & 50 & 40 & 30 & 20 & 10 & 0 & -10\end{array}$

Figure S58. ${ }^{13} \mathrm{C}$ NMR $\left(100 \mathrm{MHz}, \mathrm{CDCl}_{3}\right)$ of $\mathbf{6 c}$. 
<smiles>CC(=O)[C@@](C)(O)C(=O)Nc1cc(F)c(F)cc1F</smiles>

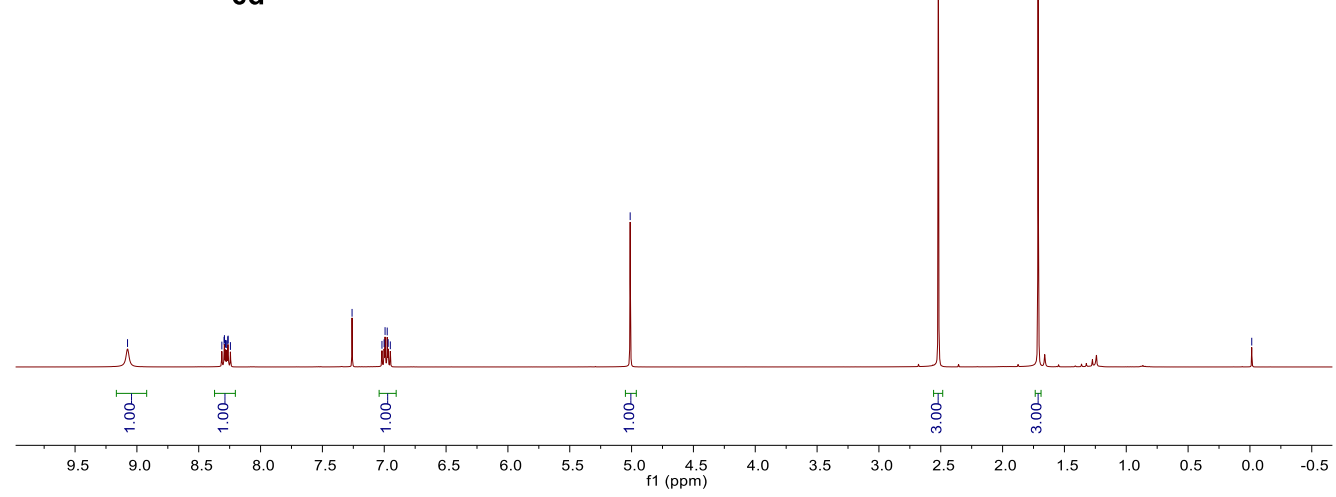

Figure S59. ${ }^{1} \mathrm{H}$ NMR $\left(400 \mathrm{MHz}, \mathrm{CDCl}_{3}\right)$ of $\mathbf{6 d}$.
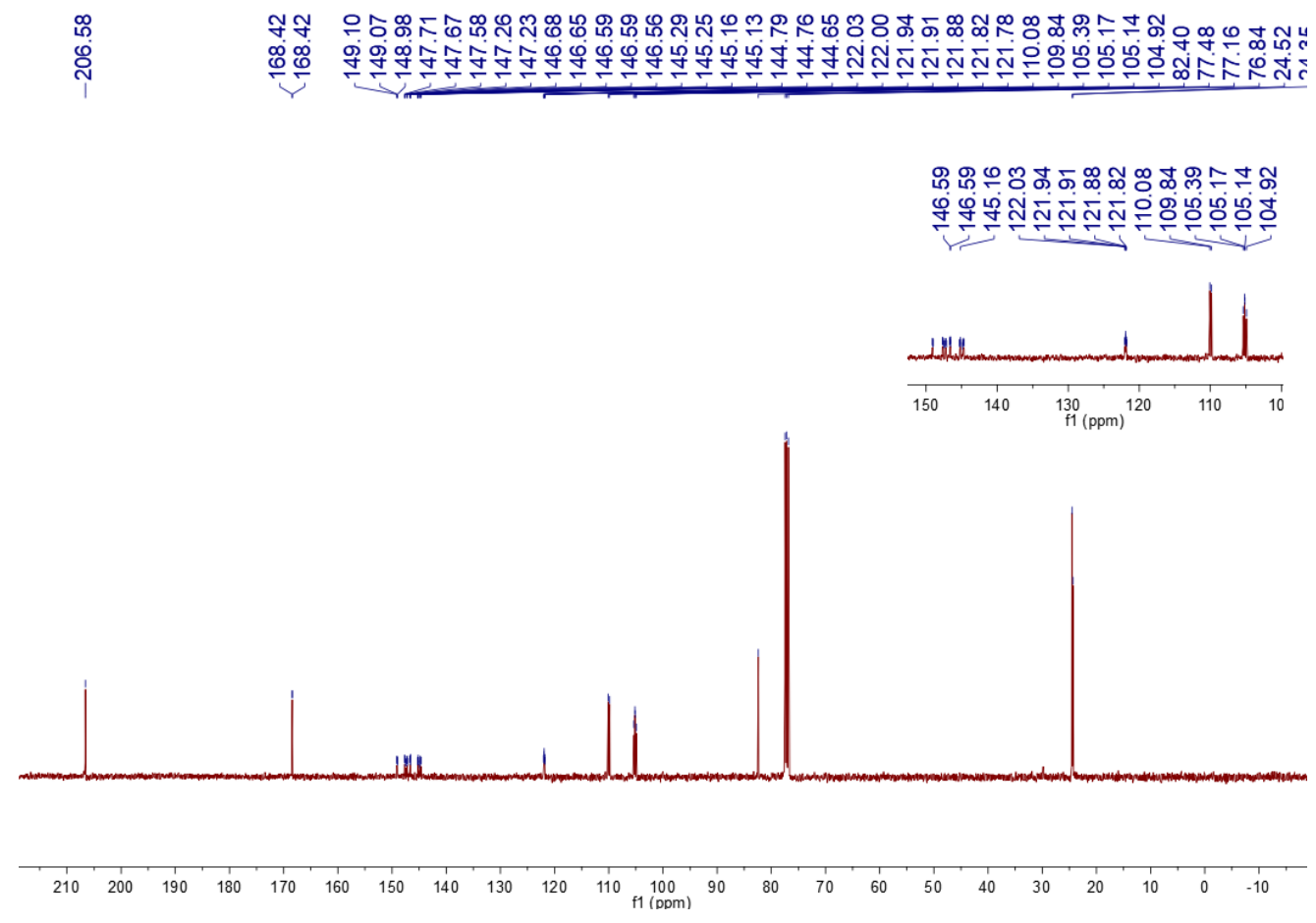

Figure S60. ${ }^{13} \mathrm{C}$ NMR $\left(100 \mathrm{MHz}, \mathrm{CDCl}_{3}\right)$ of $\mathbf{6 d}$. 

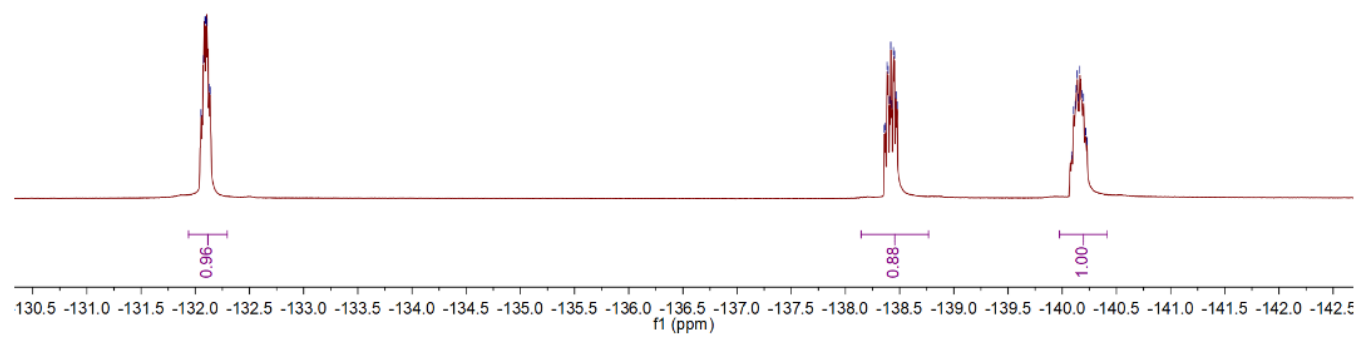

Figure S61. ${ }^{19} \mathrm{~F}$ NMR $\left(376 \mathrm{MHz}, \mathrm{CDCl}_{3}\right)$ of $\mathbf{6 d}$. 


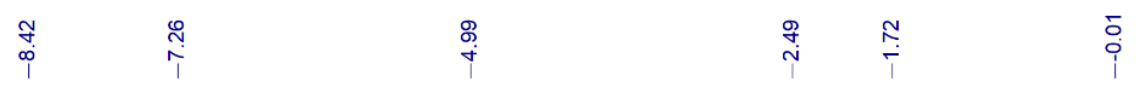<smiles>CC(=O)[C@@](C)(O)C(=O)Nc1c(F)c(F)c(F)c(F)c1F</smiles>

6e

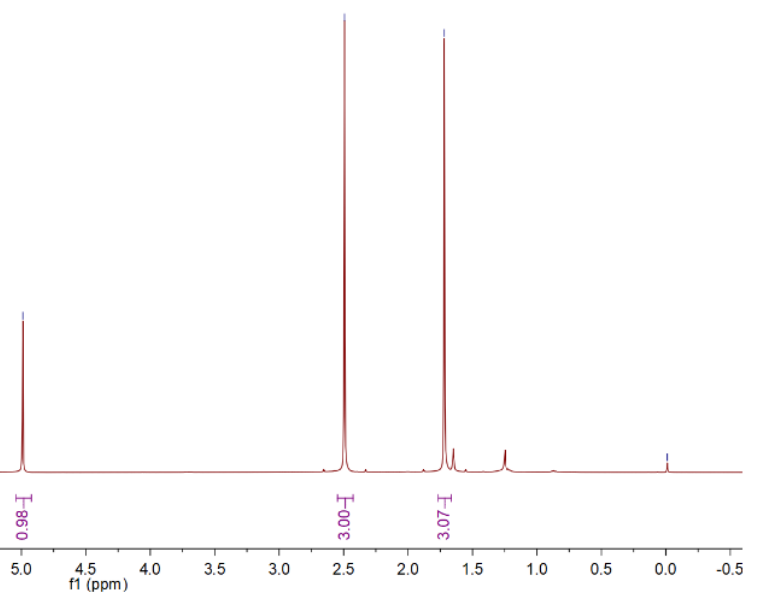

Figure S62. ${ }^{1} \mathrm{H}$ NMR $\left(400 \mathrm{MHz}, \mathrm{CDCl}_{3}\right)$ of $\mathbf{6 e}$.
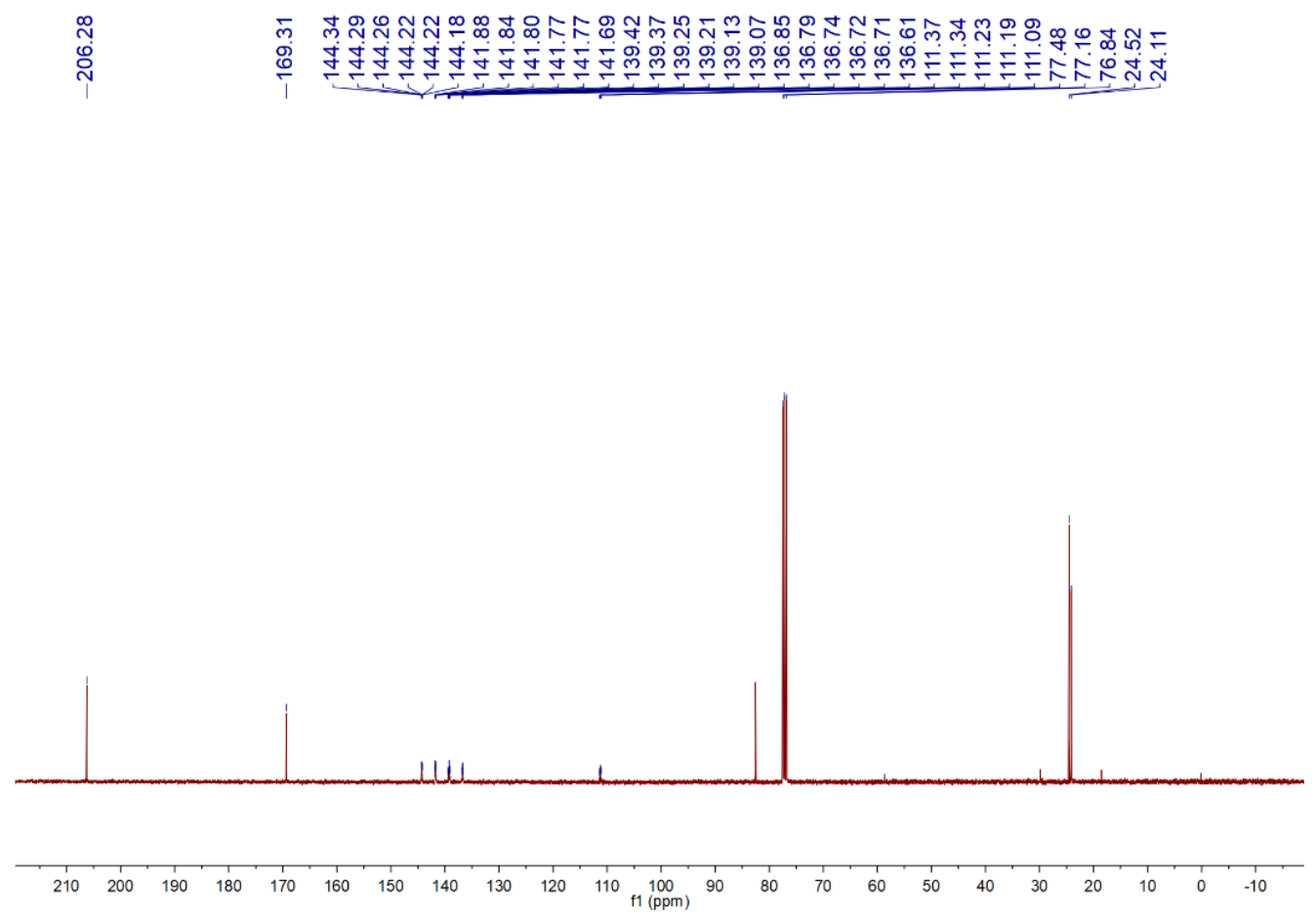

Figure S63. ${ }^{13} \mathrm{C}$ NMR $\left(100 \mathrm{MHz}, \mathrm{CDCl}_{3}\right)$ of $\mathbf{6 e}$. 


\section{œ}

寺热尃

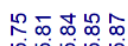

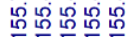

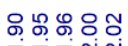

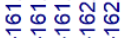

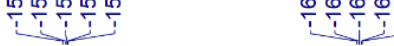

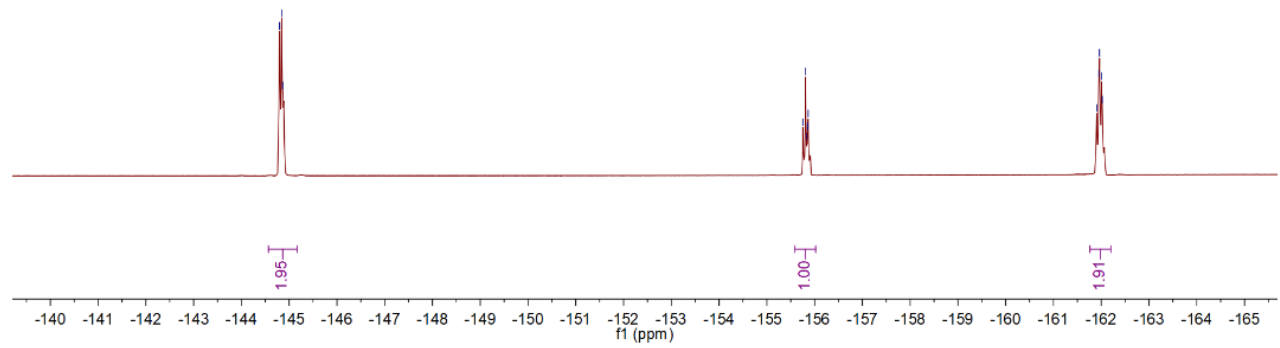

Figure S64. ${ }^{19} \mathrm{~F}$ NMR $\left(376 \mathrm{MHz}, \mathrm{CDCl}_{3}\right)$ of $\mathbf{6 e}$. 
<smiles>CC(=O)[C@](C)(O)C(=O)Nc1ccccc1C</smiles>

$6 f$

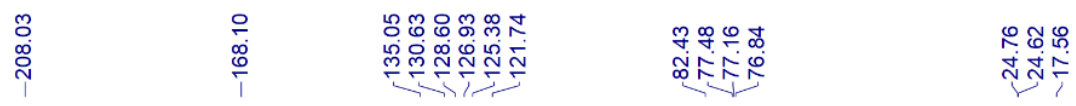

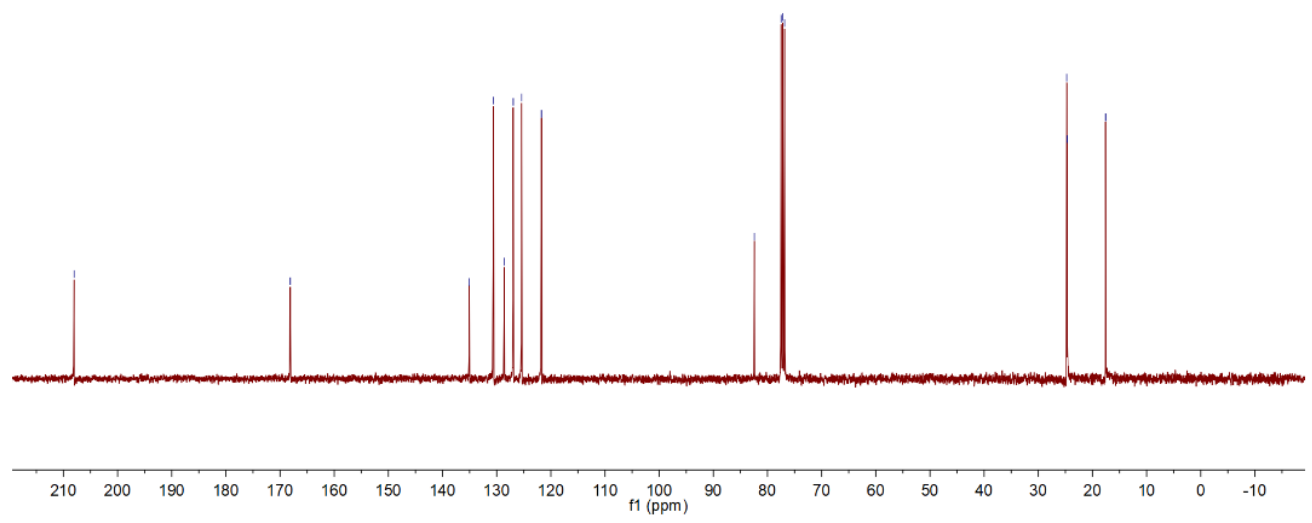

Figure S66. ${ }^{13} \mathrm{C}$ NMR $\left(100 \mathrm{MHz}, \mathrm{CDCl}_{3}\right)$ of $\mathbf{6 f}$. 


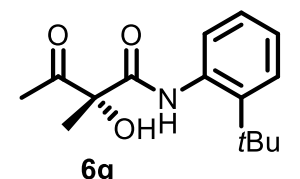

$6 \mathrm{~g}$

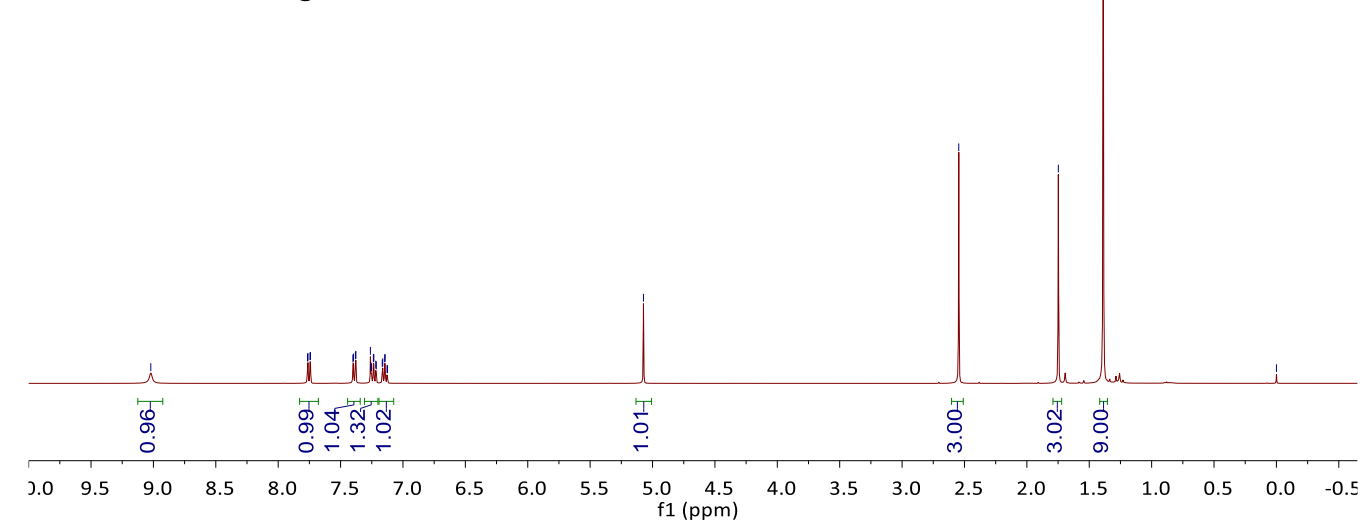

Figure S67. ${ }^{1} \mathrm{H}$ NMR $\left(400 \mathrm{MHz}, \mathrm{CDCl}_{3}\right)$ of $\mathbf{6 g}$.

\begin{tabular}{|c|c|c|c|c|}
\hline $\begin{array}{l}\stackrel{P}{0} \\
\stackrel{i}{\hat{N}}\end{array}$ & 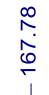 & 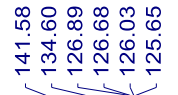 & 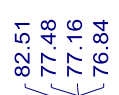 & 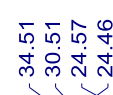 \\
\hline
\end{tabular}

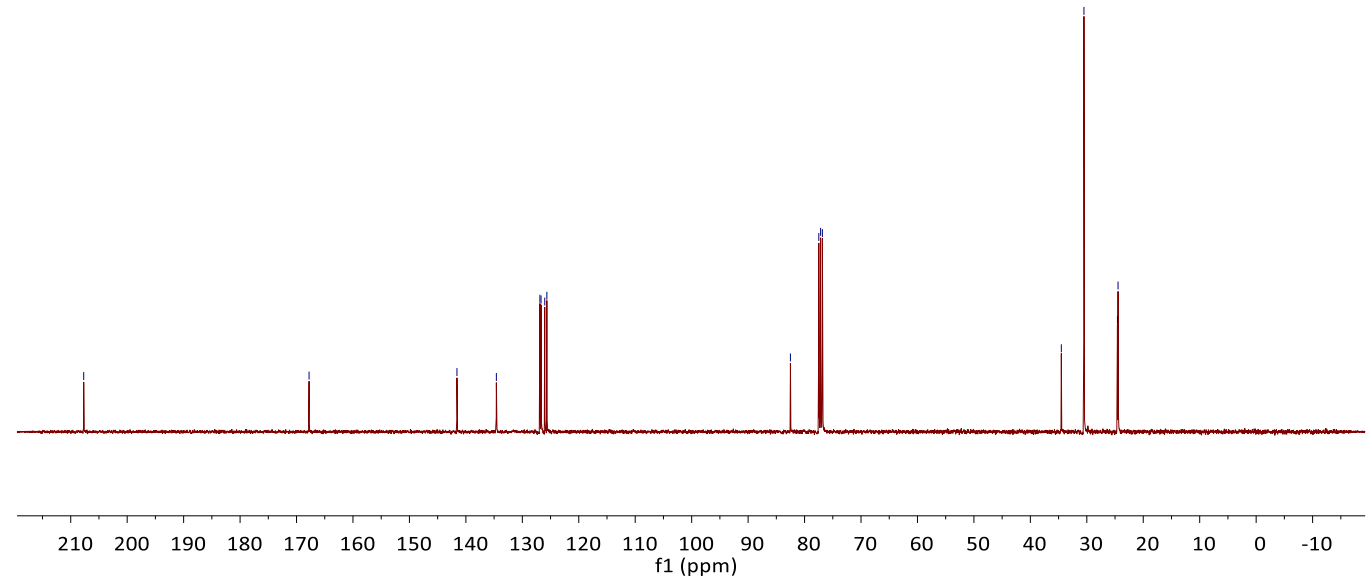

Figure S68. ${ }^{13} \mathrm{C}$ NMR $\left(100 \mathrm{MHz}, \mathrm{CDCl}_{3}\right)$ of $\mathbf{6 g}$. 
<smiles>CC(=O)[C@](C)(O)C(=O)NCc1ccccc1</smiles>

$6 \mathrm{~h}$

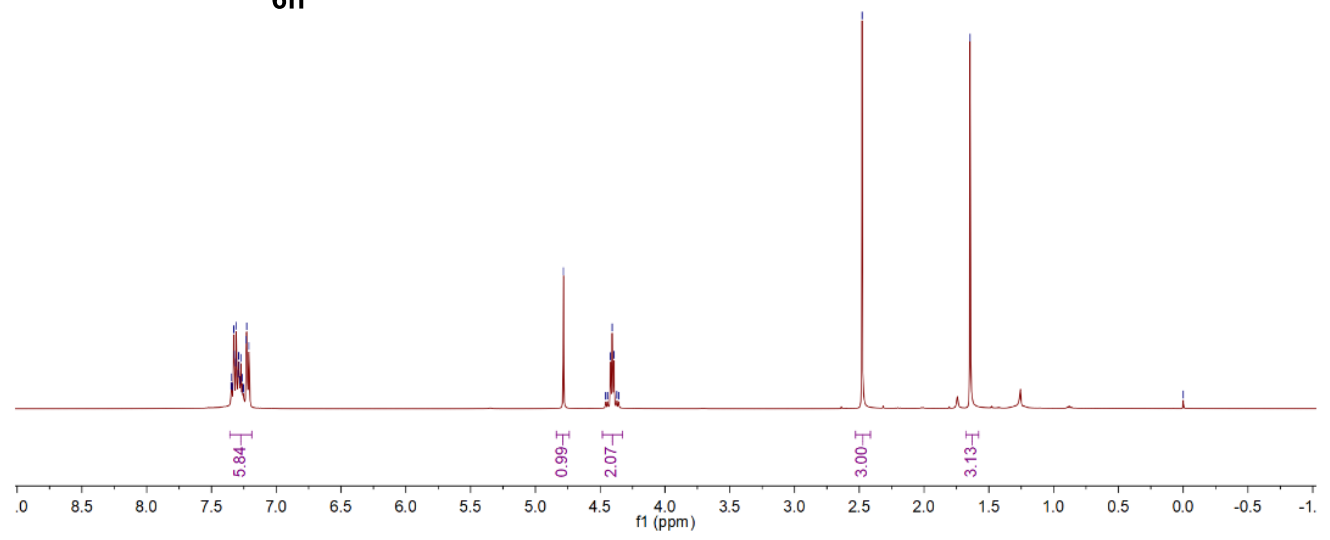

Figure S69. ${ }^{1} \mathrm{H}$ NMR $\left(400 \mathrm{MHz}, \mathrm{CDCl}_{3}\right)$ of $\mathbf{6} \mathbf{h}$.

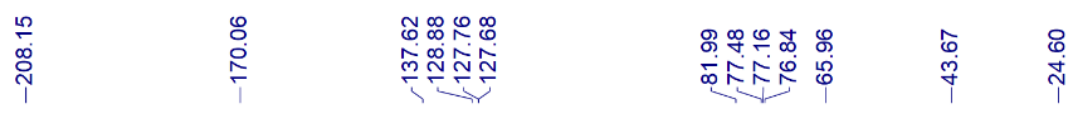
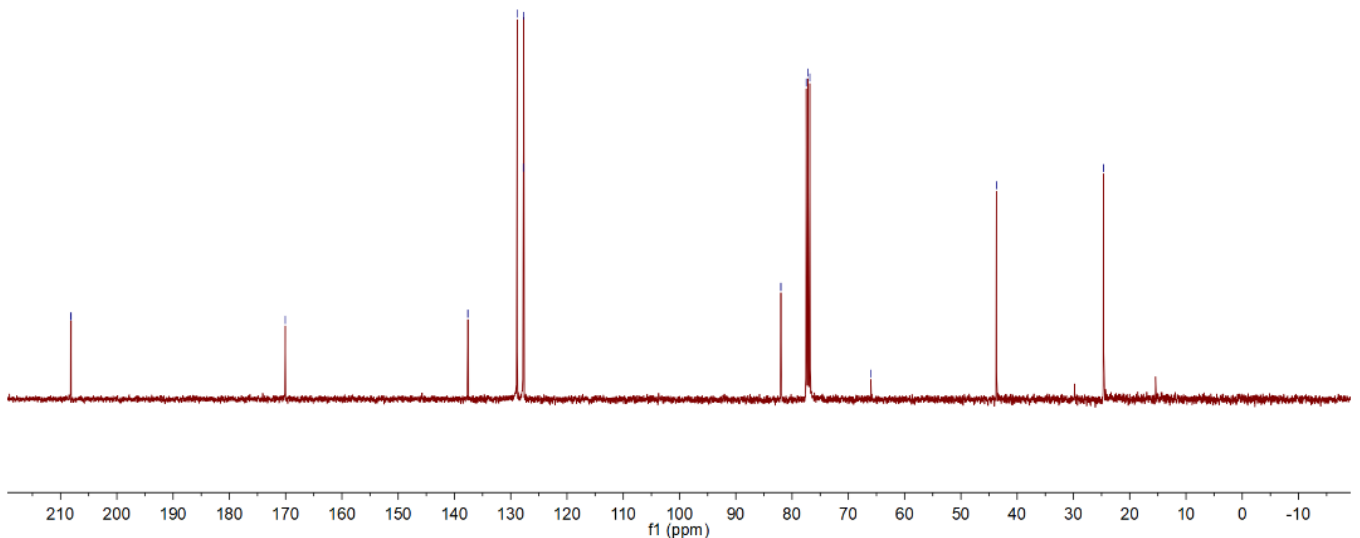

Figure S70. ${ }^{13} \mathrm{C}$ NMR $\left(100 \mathrm{MHz}, \mathrm{CDCl}_{3}\right)$ of $\boldsymbol{6} \mathbf{h}$. 
<smiles>CC[C@@](O)(C(C)=O)C(=O)Nc1ccccc1</smiles>

$6 i$

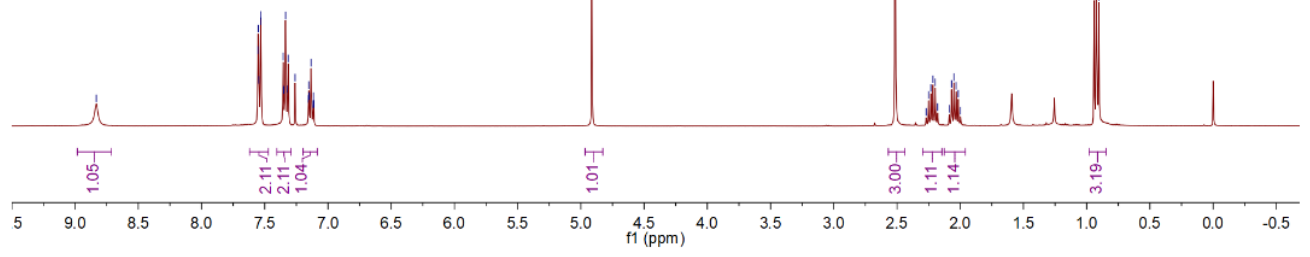

Figure S71. ${ }^{1} \mathrm{H}$ NMR $\left(400 \mathrm{MHz}, \mathrm{CDCl}_{3}\right)$ of $\mathbf{6 i}$.

\begin{tabular}{|c|c|c|c|c|}
\hline م & $\begin{array}{l}\stackrel{0}{0} \\
\stackrel{0}{0} \\
\stackrel{1}{1}\end{array}$ & 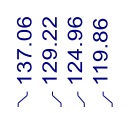 & 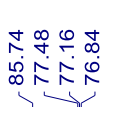 & $\begin{array}{ll}\infty & \bar{\sigma} \\
\infty & \dot{p} \\
\dot{p} & \stackrel{\sim}{j} \\
1 & 1\end{array}$ \\
\hline
\end{tabular}

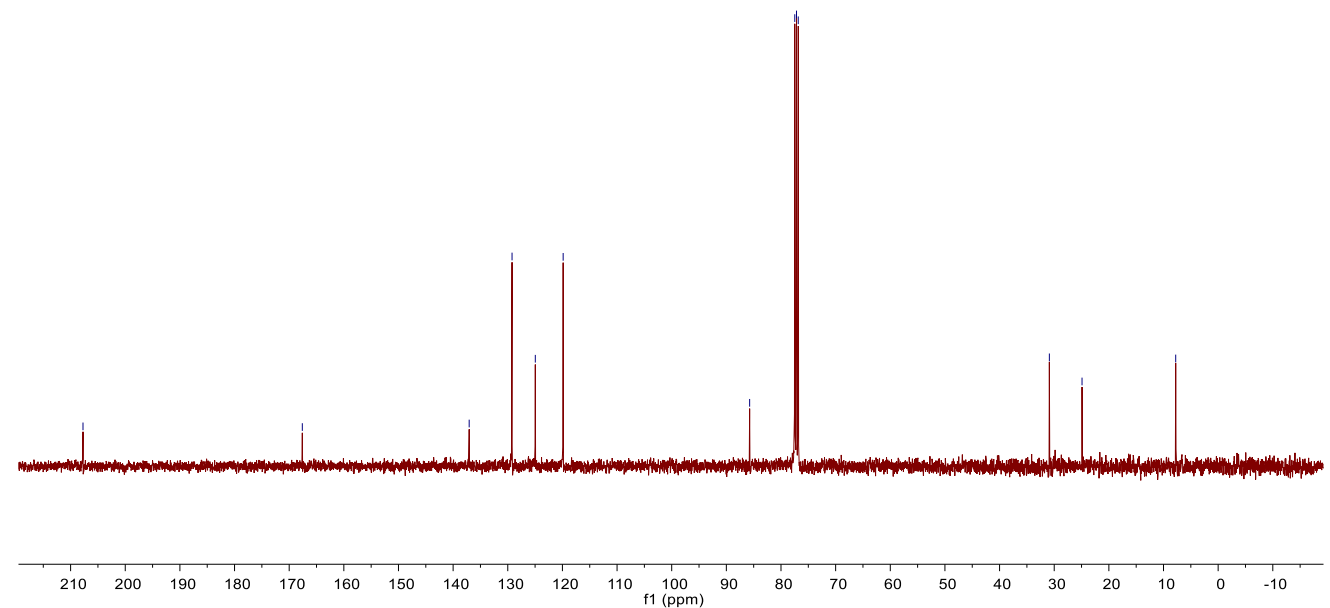

Figure S72. ${ }^{13} \mathrm{C}$ NMR $\left(100 \mathrm{MHz}, \mathrm{CDCl}_{3}\right)$ of $\mathbf{6 i}$. 
<smiles>CC(=O)[C@@](O)(C(=O)O)C(=O)Nc1ccccc1</smiles>

6j

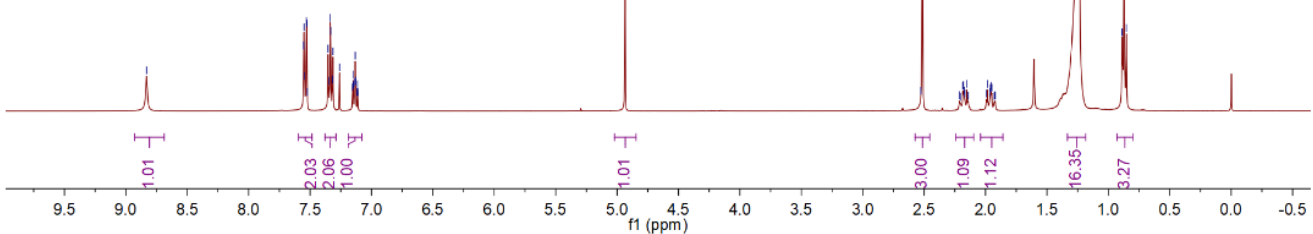

Figure S73. ${ }^{1} \mathrm{H}$ NMR $\left(400 \mathrm{MHz}, \mathrm{CDCl}_{3}\right)$ of $\mathbf{6 j}$.

\begin{tabular}{|c|c|c|c|}
\hline $\begin{array}{l}\stackrel{\leftrightarrow}{0} \\
\stackrel{0}{0}\end{array}$ & 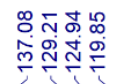 & 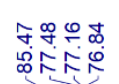 & 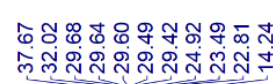 \\
\hline
\end{tabular}

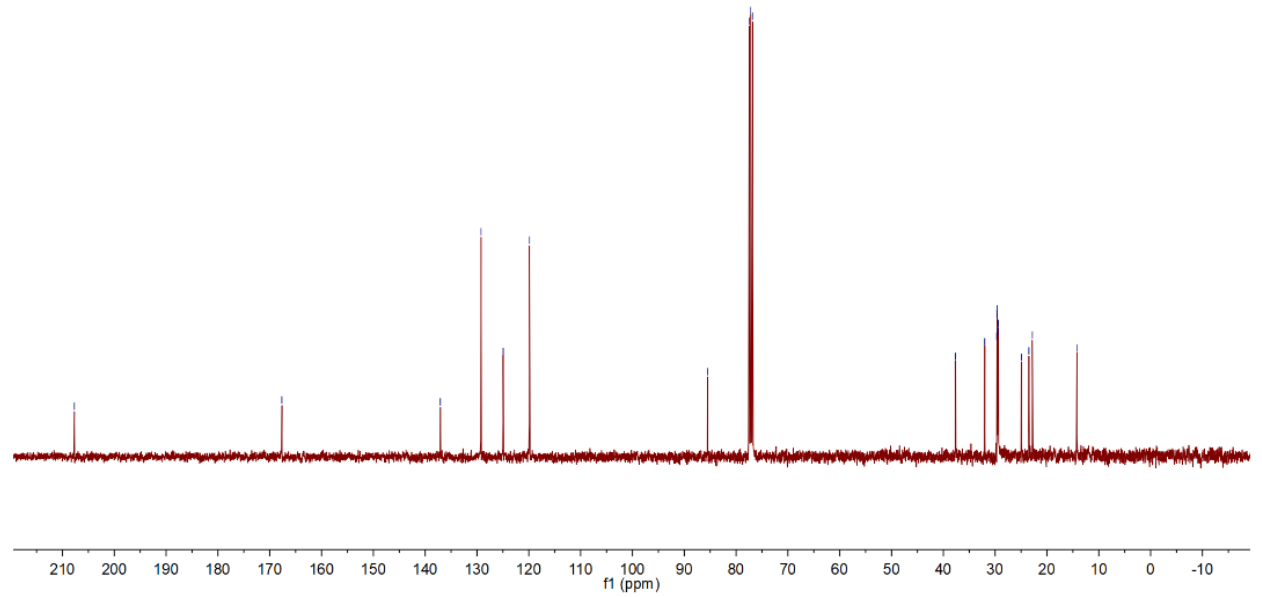

Figure S74. ${ }^{13} \mathrm{C}$ NMR $\left(100 \mathrm{MHz}, \mathrm{CDCl}_{3}\right)$ of $\mathbf{6 j}$. 
<smiles>CCC(=O)[C@](C)(O)C(=O)Nc1ccccc1Cl</smiles>

6k

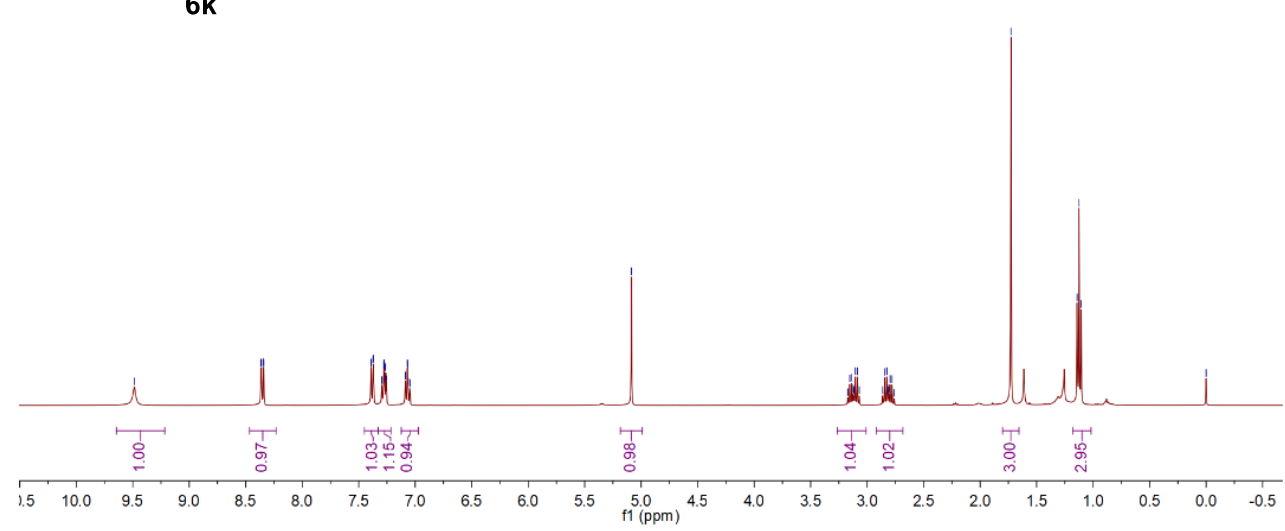

Figure S75. ${ }^{1} \mathrm{H}$ NMR $\left(400 \mathrm{MHz}, \mathrm{CDCl}_{3}\right)$ of $\mathbf{6 k}$.

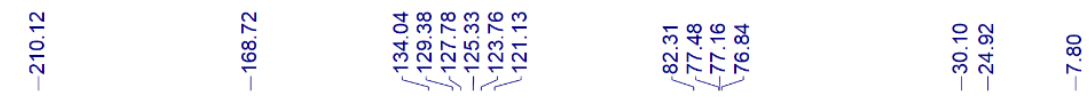

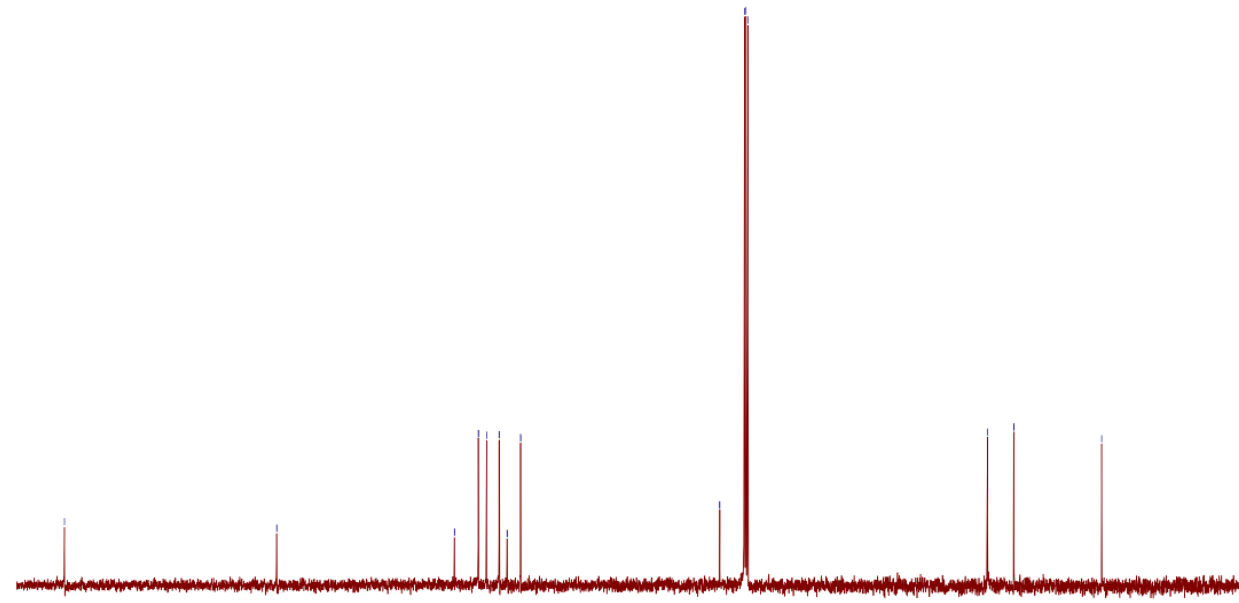

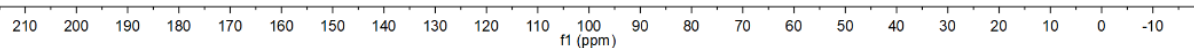

Figure S76. ${ }^{13} \mathrm{C}$ NMR $\left(100 \mathrm{MHz}, \mathrm{CDCl}_{3}\right)$ of $\mathbf{6 k}$. 
<smiles>O=C1CCC[C@H](O)C1(O)C(=O)Nc1ccccc1</smiles>

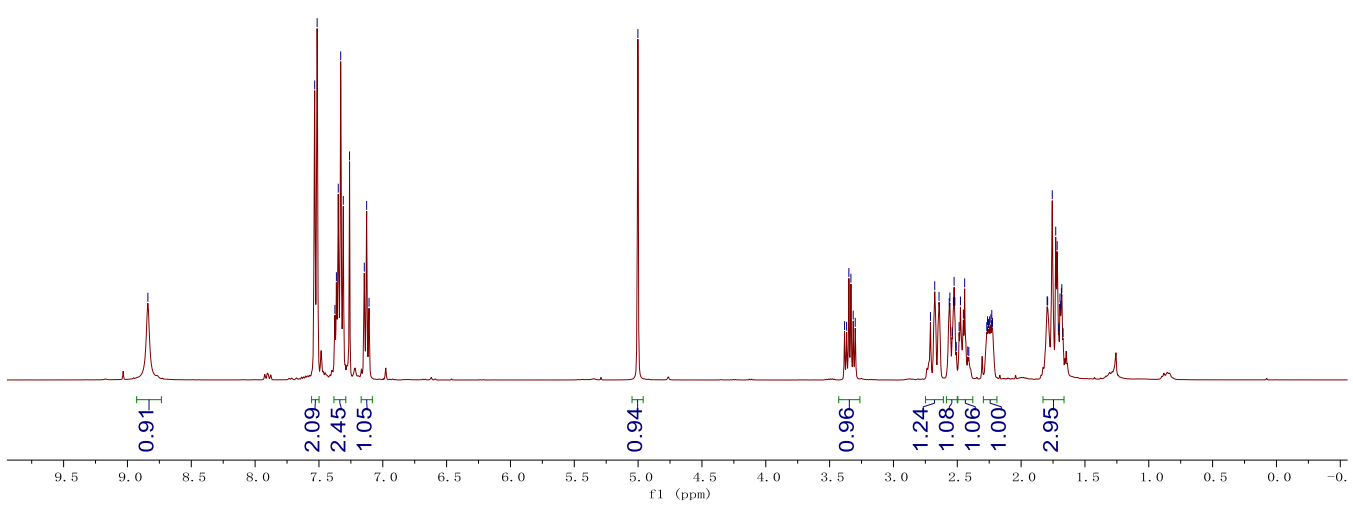

Figure S77. ${ }^{1} \mathrm{H}$ NMR $\left(400 \mathrm{MHz}, \mathrm{CDCl}_{3}\right)$ of 61 .

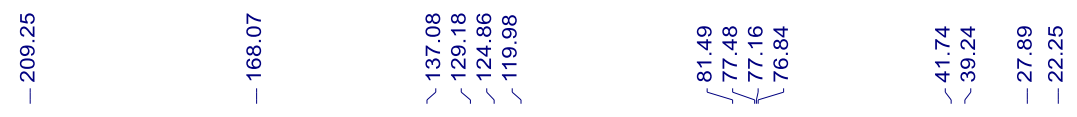

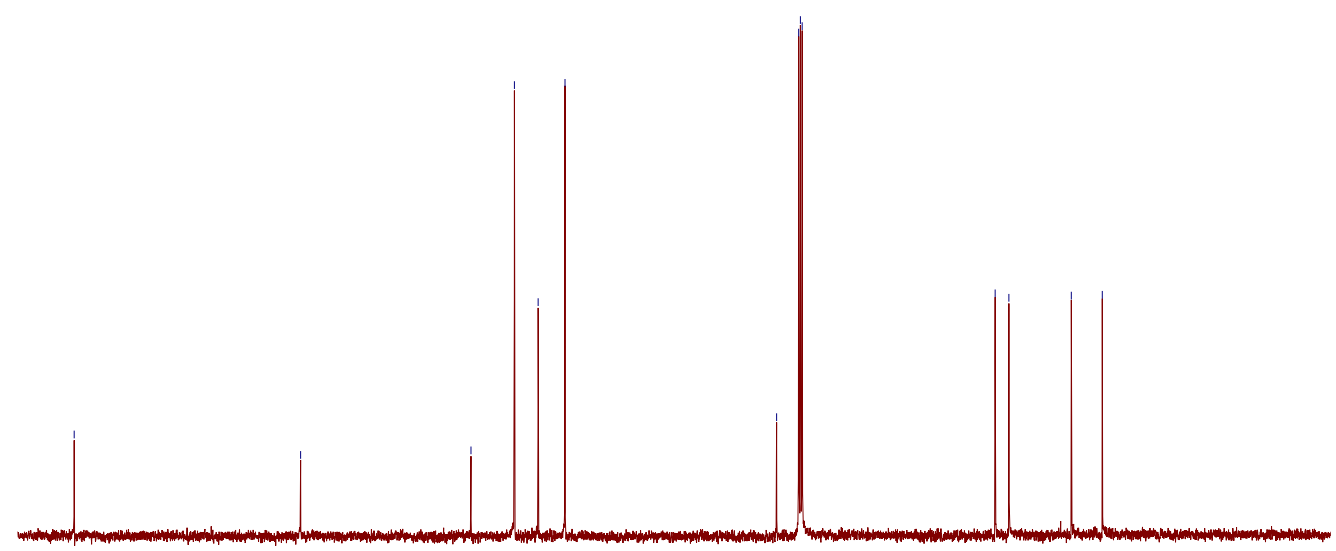

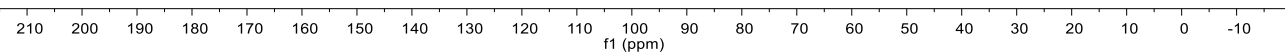

Figure S78. ${ }^{13} \mathrm{C}$ NMR $\left(100 \mathrm{MHz}, \mathrm{CDCl}_{3}\right)$ of $\mathbf{6 l}$. 
<smiles>Cc1ccc(NC(=O)[C@@]2(O)CCCCC2=O)cc1</smiles>

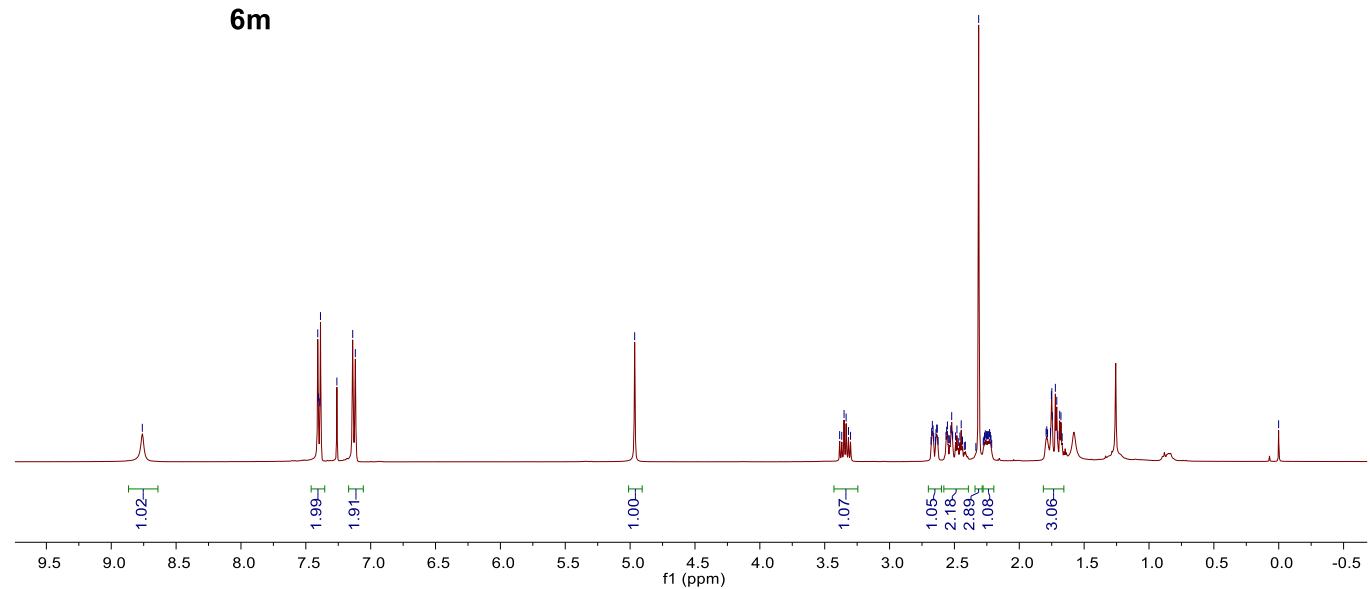

Figure S79. ${ }^{1} \mathrm{H}$ NMR $\left(400 \mathrm{MHz}, \mathrm{CDCl}_{3}\right)$ of $\mathbf{6 m}$.

\begin{tabular}{|c|c|c|c|}
\hline & 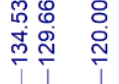 & 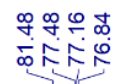 & 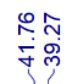 \\
\hline
\end{tabular}

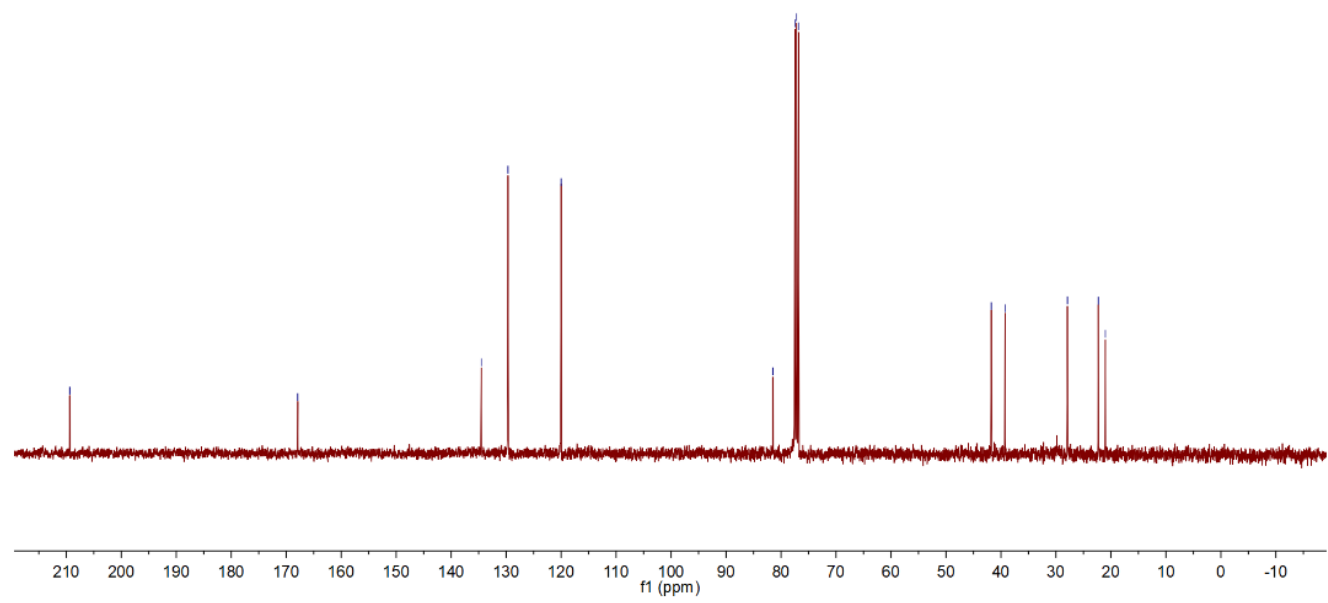

Figure S80. ${ }^{13} \mathrm{C}$ NMR $\left(100 \mathrm{MHz}, \mathrm{CDCl}_{3}\right)$ of $\mathbf{6 m}$. 


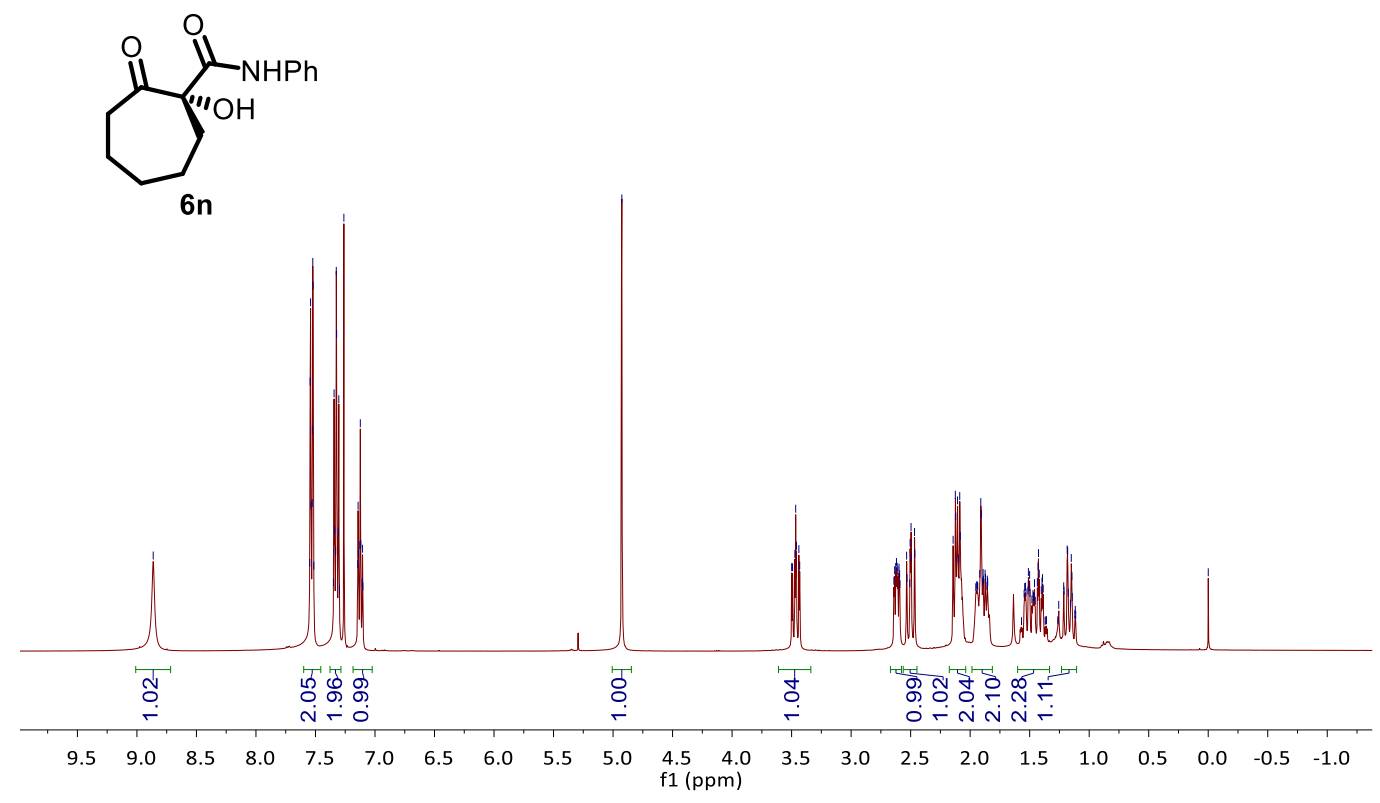

Figure S81. ${ }^{1} \mathrm{H}$ NMR $\left(400 \mathrm{MHz}, \mathrm{CDCl}_{3}\right)$ of $\mathbf{6 n}$.

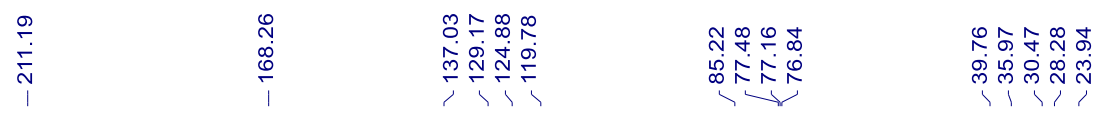
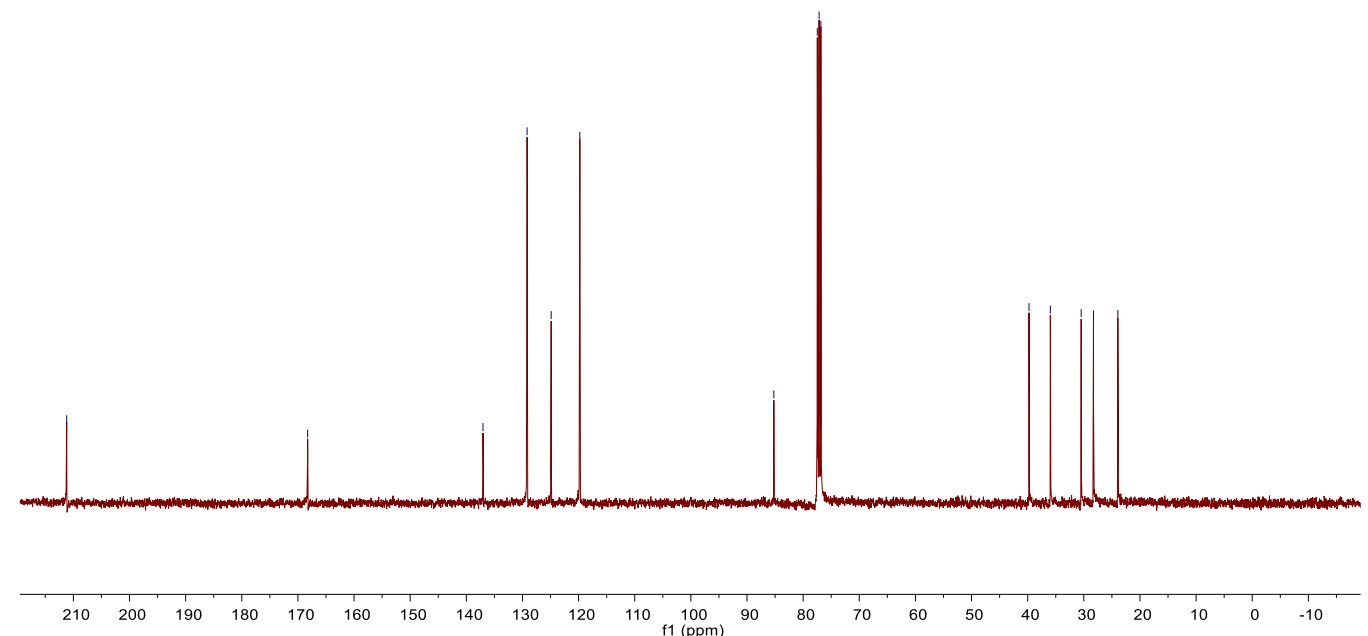

Figure S82. ${ }^{13} \mathrm{C}$ NMR $\left(100 \mathrm{MHz}, \mathrm{CDCl}_{3}\right)$ of $\mathbf{6 n}$. 
<smiles>COC(=O)[C@H](Cc1ccccc1)NC(=O)[C@](C)(O)C(C)=O</smiles>

60

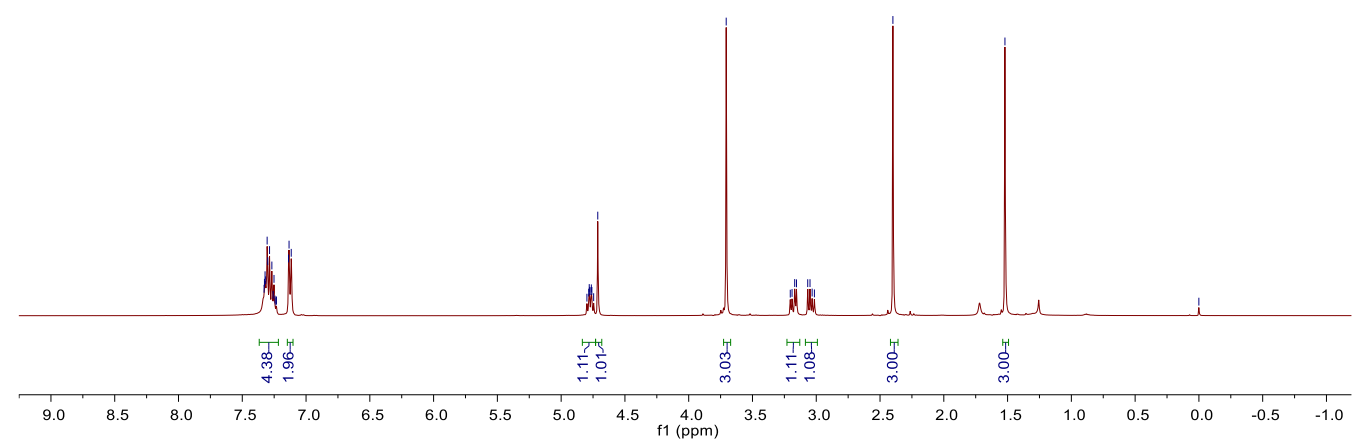

Figure S83. ${ }^{1} \mathrm{H}$ NMR $\left(400 \mathrm{MHz}, \mathrm{CDCl}_{3}\right)$ of $\mathbf{6 0}$.

\begin{tabular}{|c|c|c|c|c|c|}
\hline 㐫 & 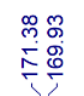 & 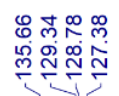 & 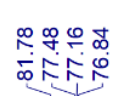 & 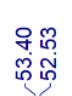 & $\underset{\infty}{\mp}$ \\
\hline
\end{tabular}

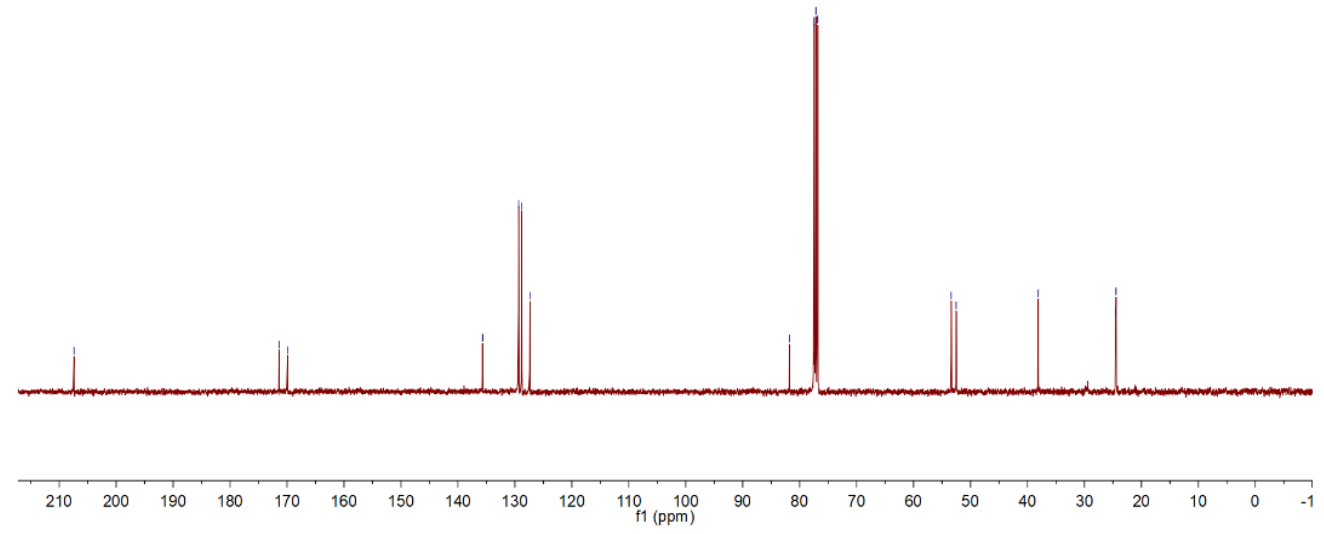

Figure S84. ${ }^{13} \mathrm{C}$ NMR $\left(100 \mathrm{MHz}, \mathrm{CDCl}_{3}\right)$ of $\mathbf{6 o}$. 


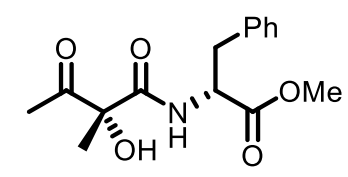

$6 p$

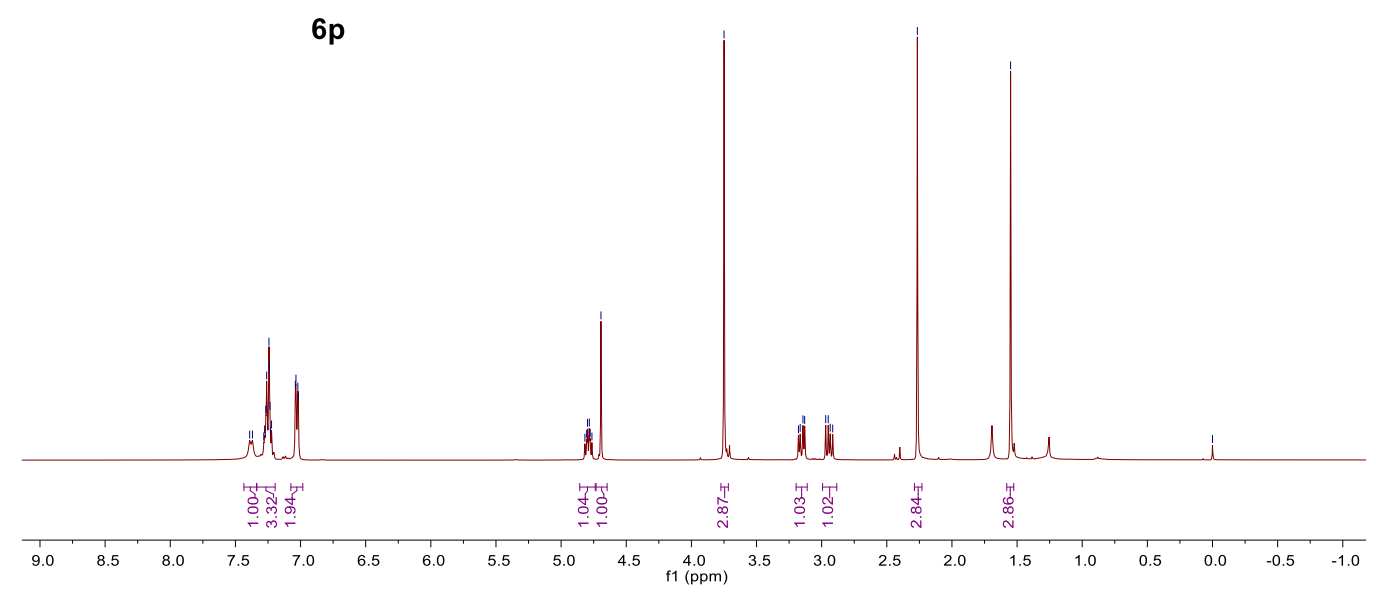

Figure S85. ${ }^{1} \mathrm{H}$ NMR $\left(400 \mathrm{MHz}, \mathrm{CDCl}_{3}\right)$ of $\mathbf{6 p}$.

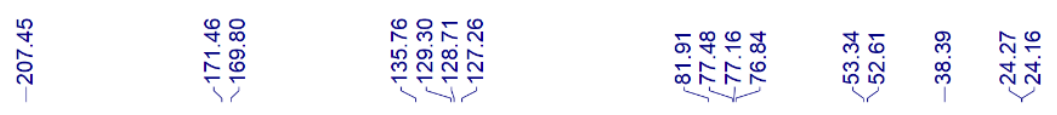

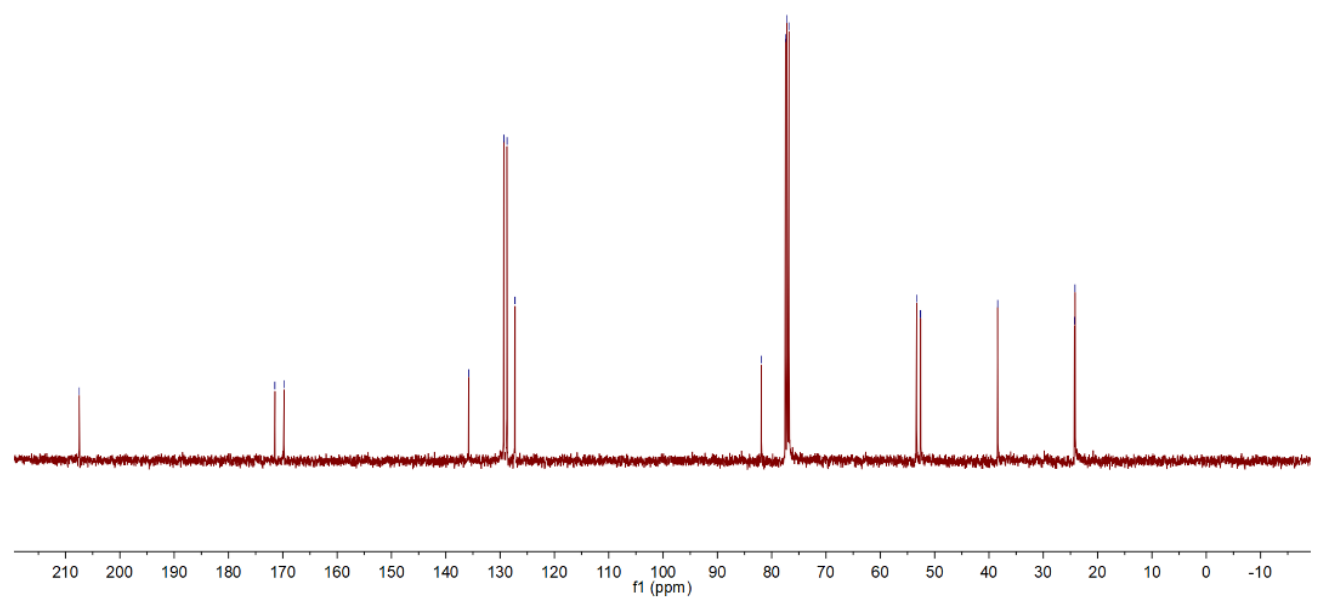

Figure S86. ${ }^{13} \mathrm{C}$ NMR $\left(100 \mathrm{MHz}, \mathrm{CDCl}_{3}\right)$ of $\mathbf{6 p}$. 

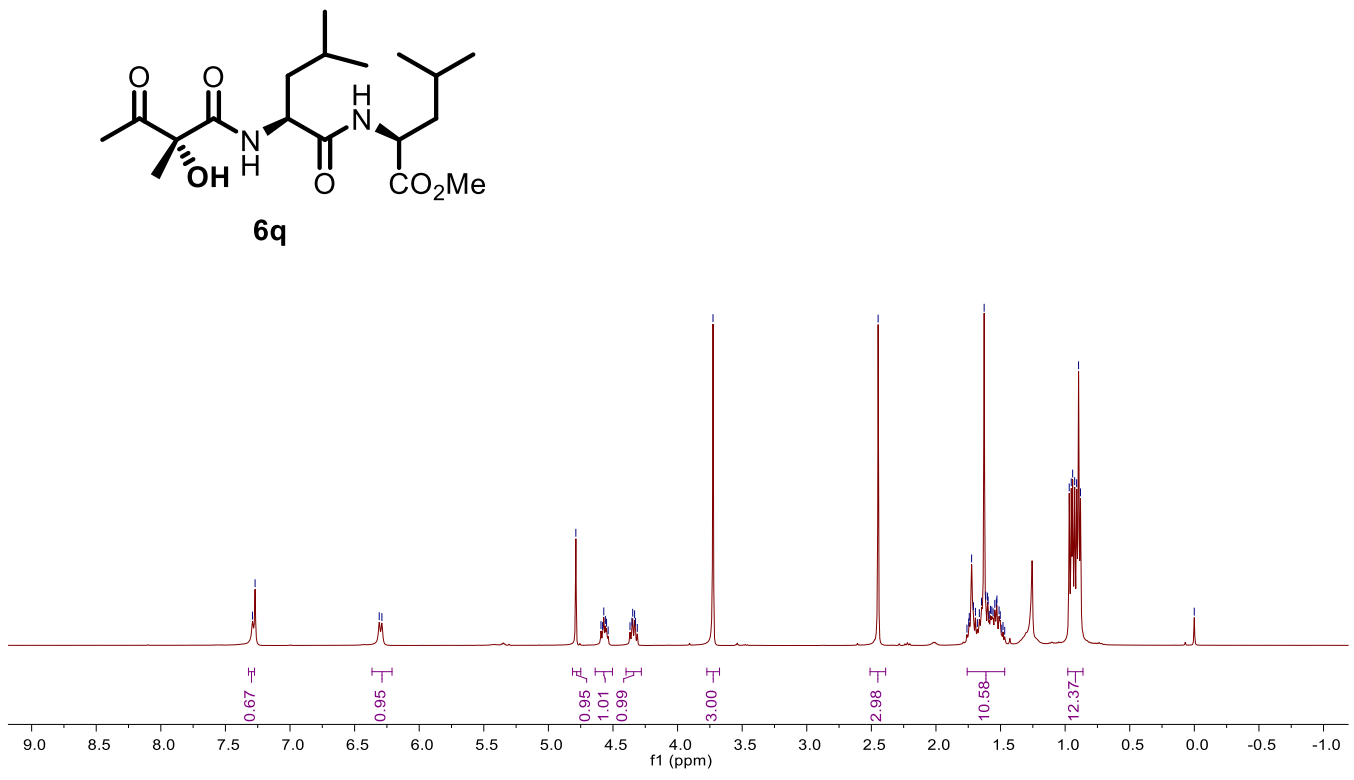

Figure S87. ${ }^{1} \mathrm{H}$ NMR $\left(400 \mathrm{MHz}, \mathrm{CDCl}_{3}\right)$ of $\mathbf{6 q}$.

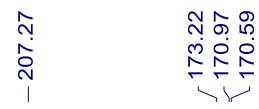

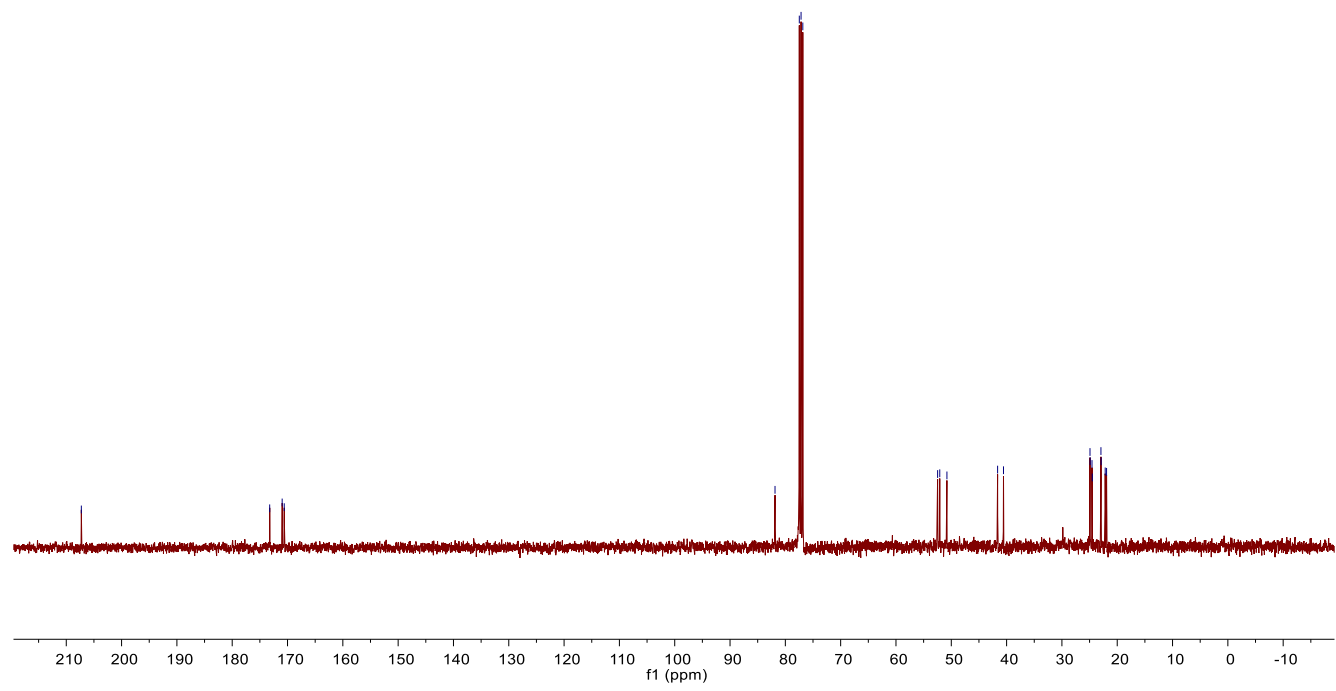

Figure S88. ${ }^{13} \mathrm{C}$ NMR $\left(100 \mathrm{MHz}, \mathrm{CDCl}_{3}\right)$ of $\mathbf{6 q}$. 
<smiles>CC(=O)[C@](C)(O)C(=O)OCCC12CCC(C1)C(C)(C)C2</smiles>

$6 r$

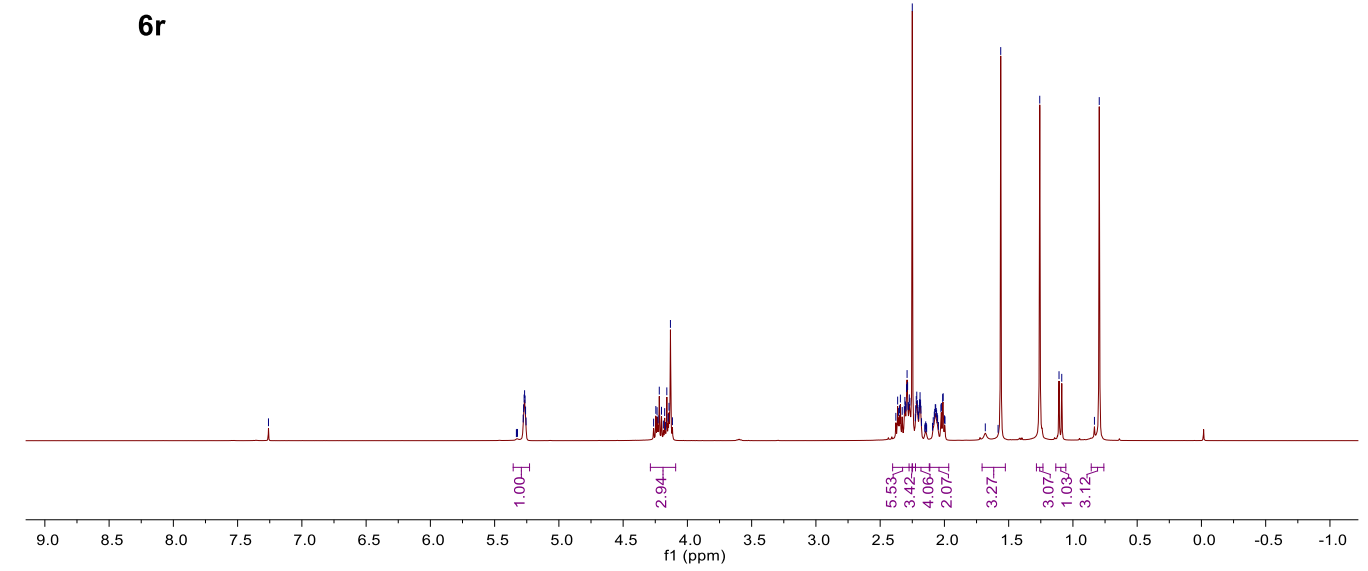

Figure S89. ${ }^{1} \mathrm{H}$ NMR $\left(400 \mathrm{MHz}, \mathrm{CDCl}_{3}\right)$ of $\mathbf{6 r}$.

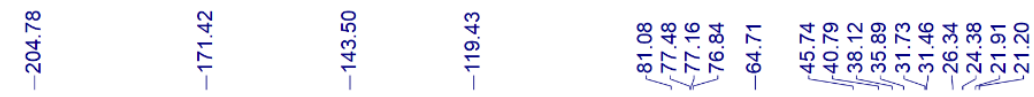

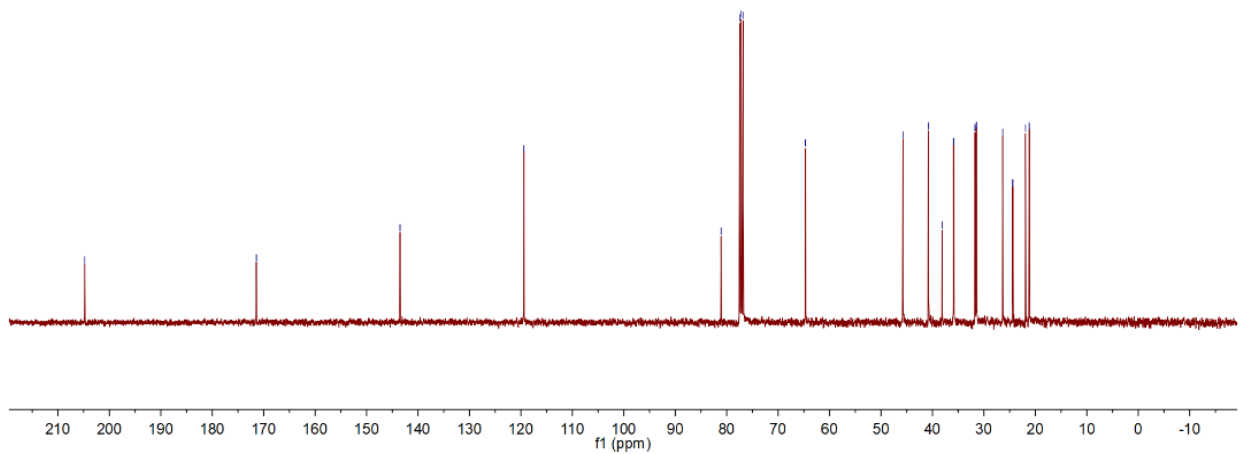

Figure S90. ${ }^{13} \mathrm{C}$ NMR $\left(100 \mathrm{MHz}, \mathrm{CDCl}_{3}\right)$ of $\mathbf{6 r}$. 


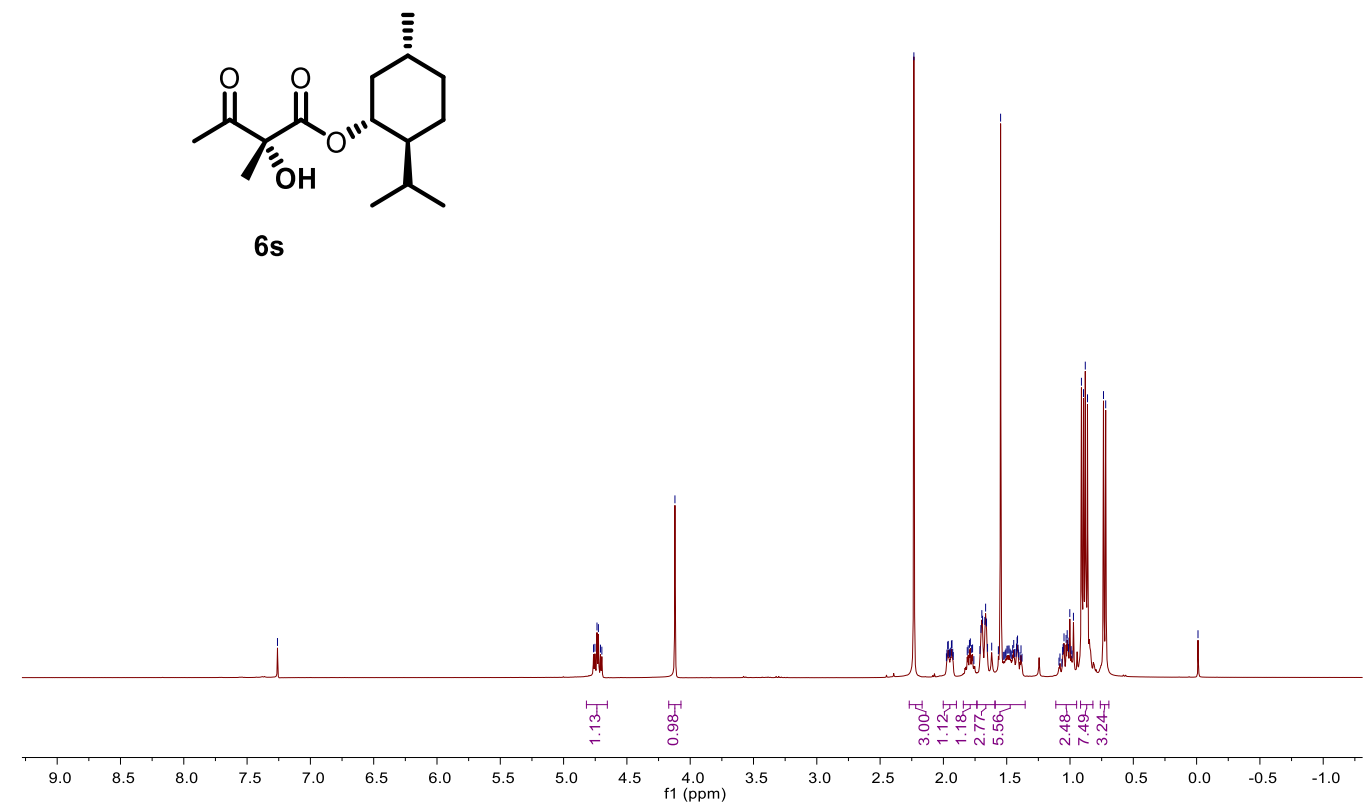

Figure S91. ${ }^{1} \mathrm{H}$ NMR $\left(400 \mathrm{MHz}, \mathrm{CDCl}_{3}\right)$ of $\mathbf{6 s}$.

\begin{tabular}{|c|c|c|}
\hline 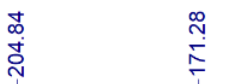 & 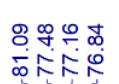 & 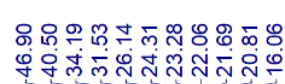 \\
\hline
\end{tabular}

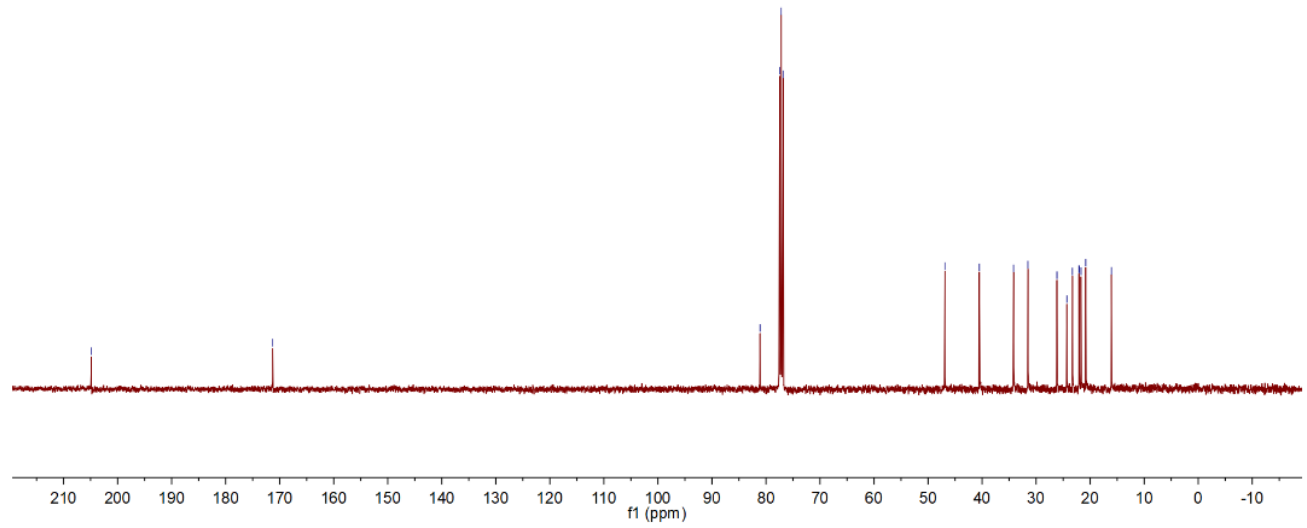

Figure S92. ${ }^{13} \mathrm{C}$ NMR $\left(100 \mathrm{MHz}, \mathrm{CDCl}_{3}\right)$ of $\mathbf{6 s}$. 


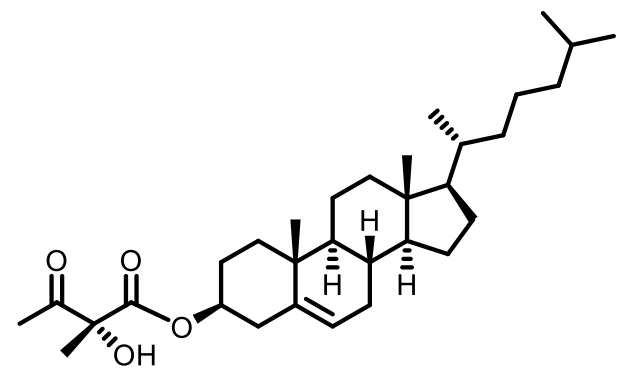

$6 t$

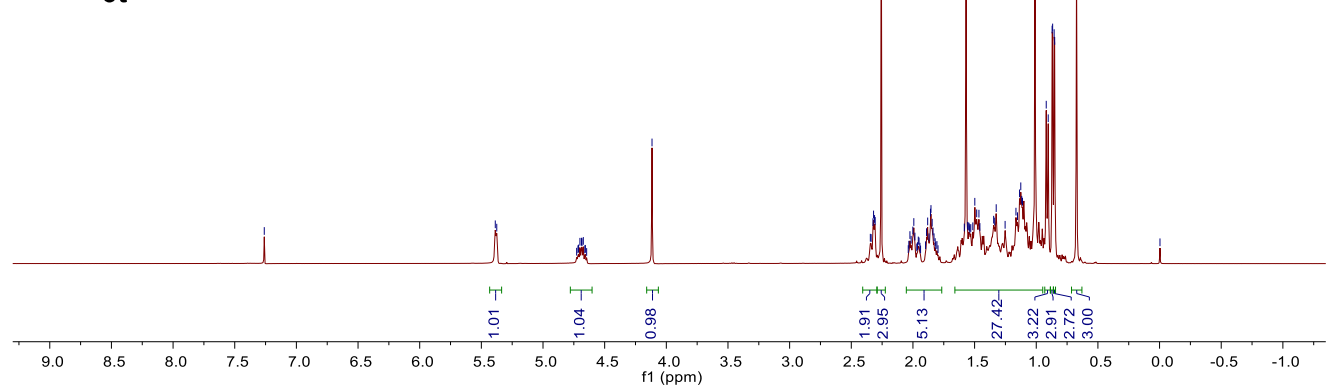

Figure S93. ${ }^{1} \mathrm{H}$ NMR $\left(400 \mathrm{MHz}, \mathrm{CDCl}_{3}\right)$ of $\mathbf{6 t}$.
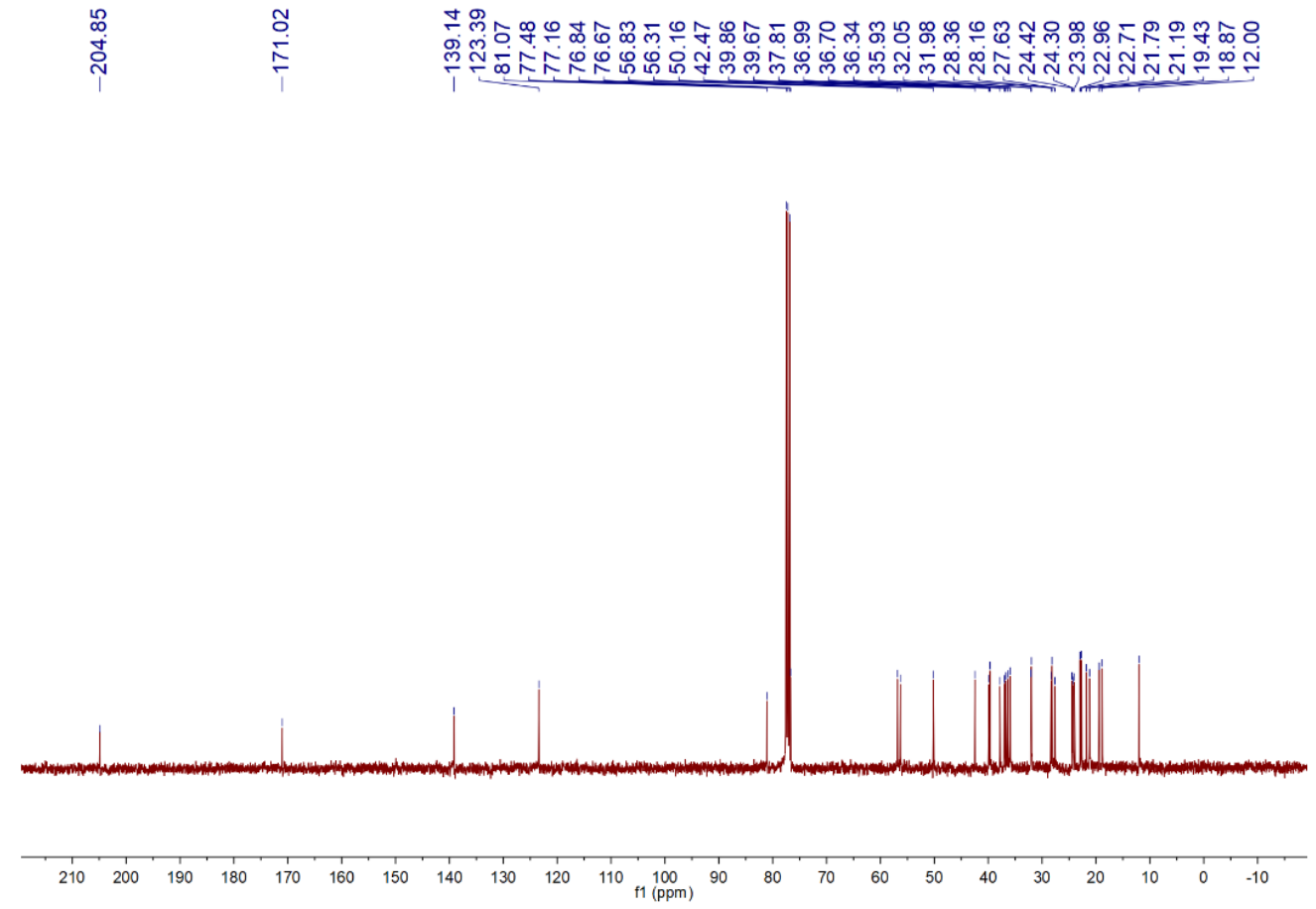

Figure S94. ${ }^{13} \mathrm{C}$ NMR $\left(100 \mathrm{MHz}, \mathrm{CDCl}_{3}\right)$ of $\mathbf{6 t}$. 


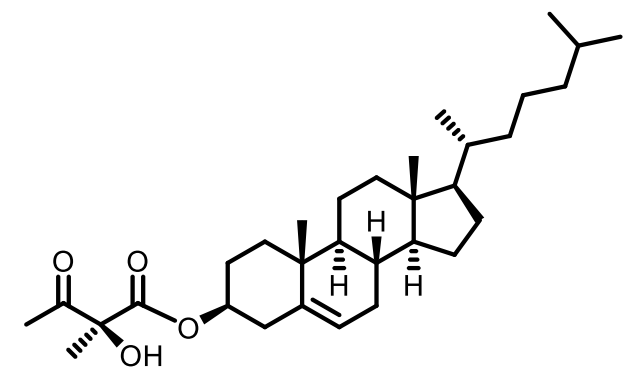

6u

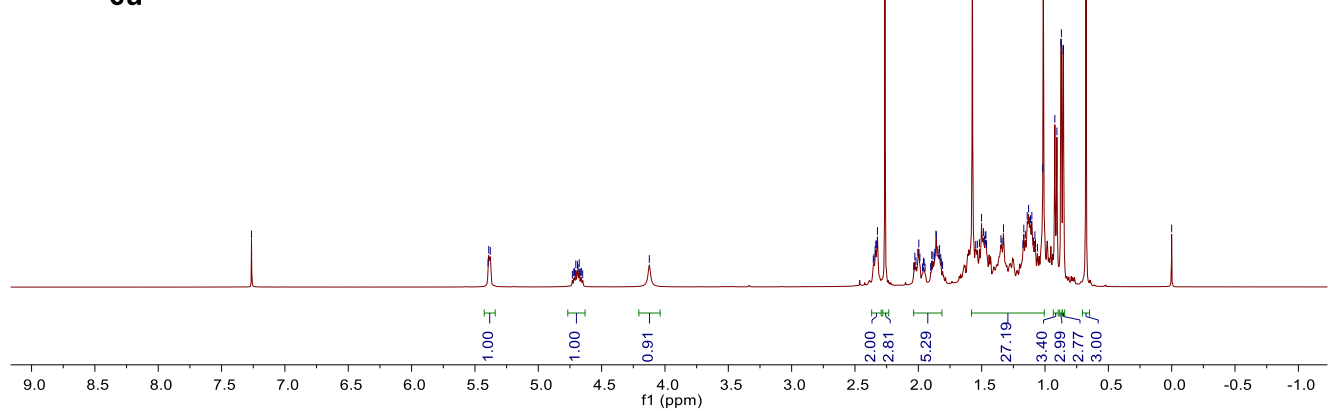

Figure S95. ${ }^{1} \mathrm{H}$ NMR $\left(400 \mathrm{MHz}, \mathrm{CDCl}_{3}\right)$ of $\mathbf{6 u}$.
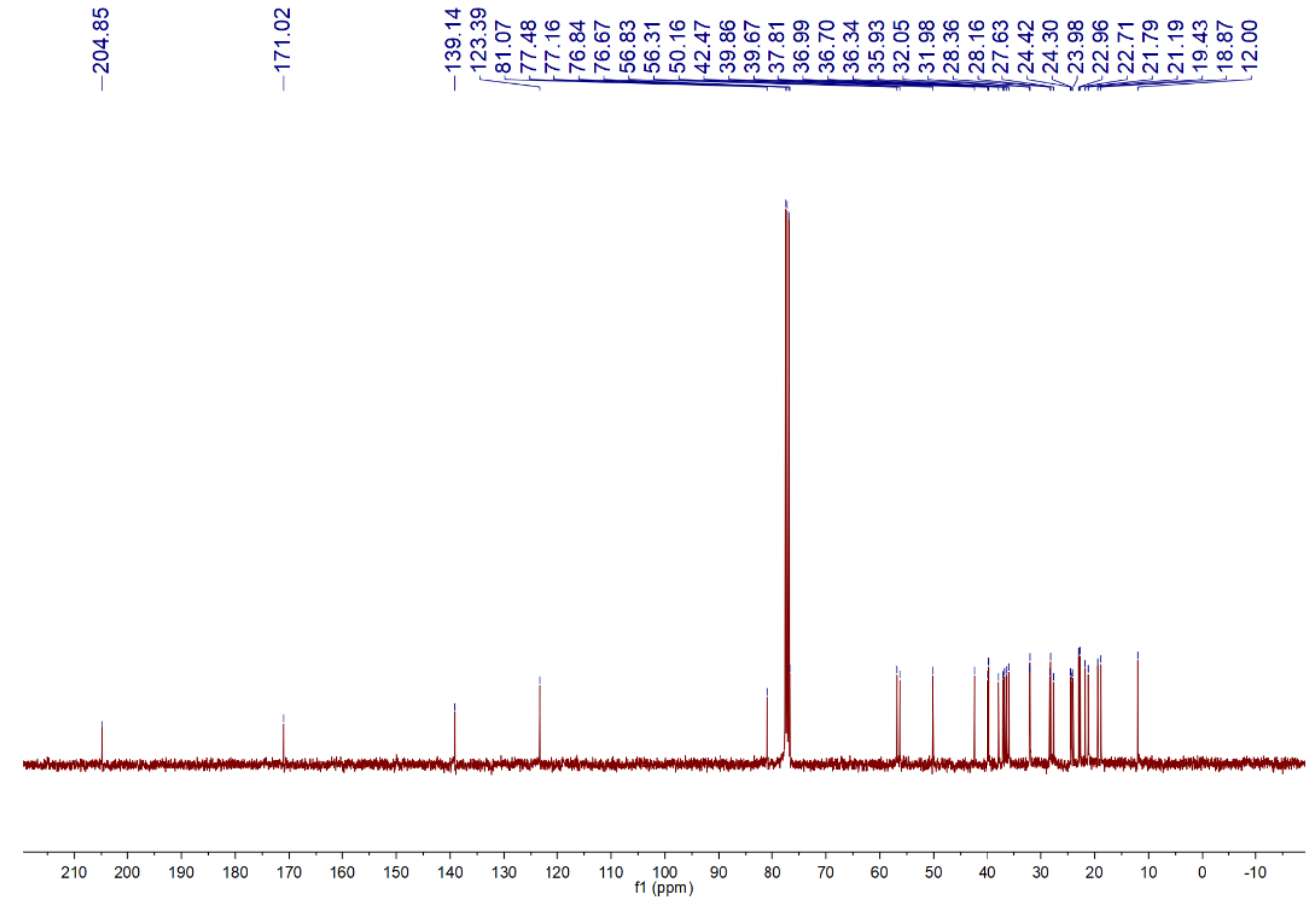

Figure S96. ${ }^{13} \mathrm{C} \mathrm{NMR}\left(100 \mathrm{MHz}, \mathrm{CDCl}_{3}\right)$ of $\mathbf{6 u}$. 
<smiles>CC[C@@](C)(O)[C@@](C)(O)C(=O)Br</smiles>

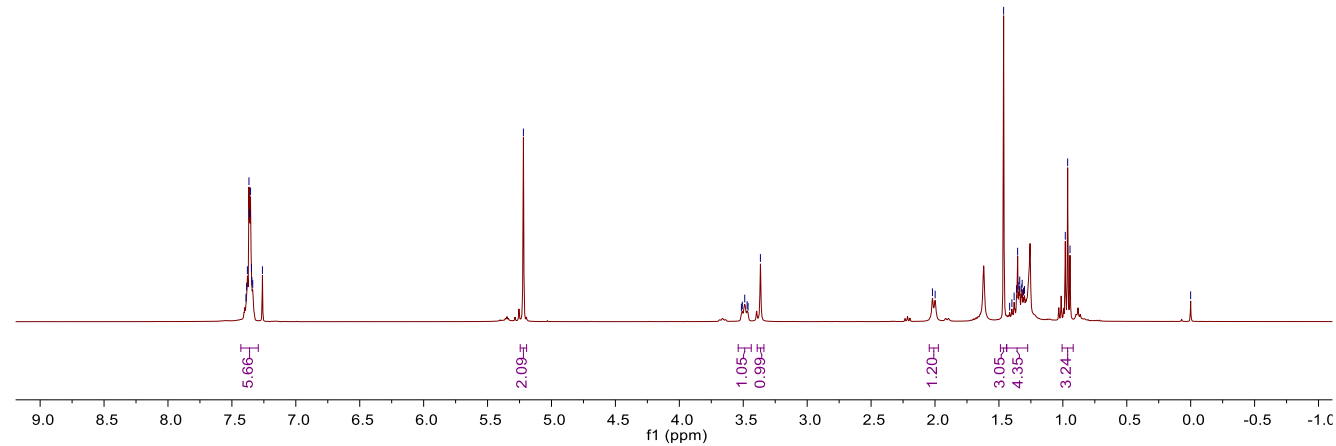

Figure S97. ${ }^{1} \mathrm{H}$ NMR $\left(400 \mathrm{MHz}, \mathrm{CDCl}_{3}\right)$ of $\mathbf{8 a}$.

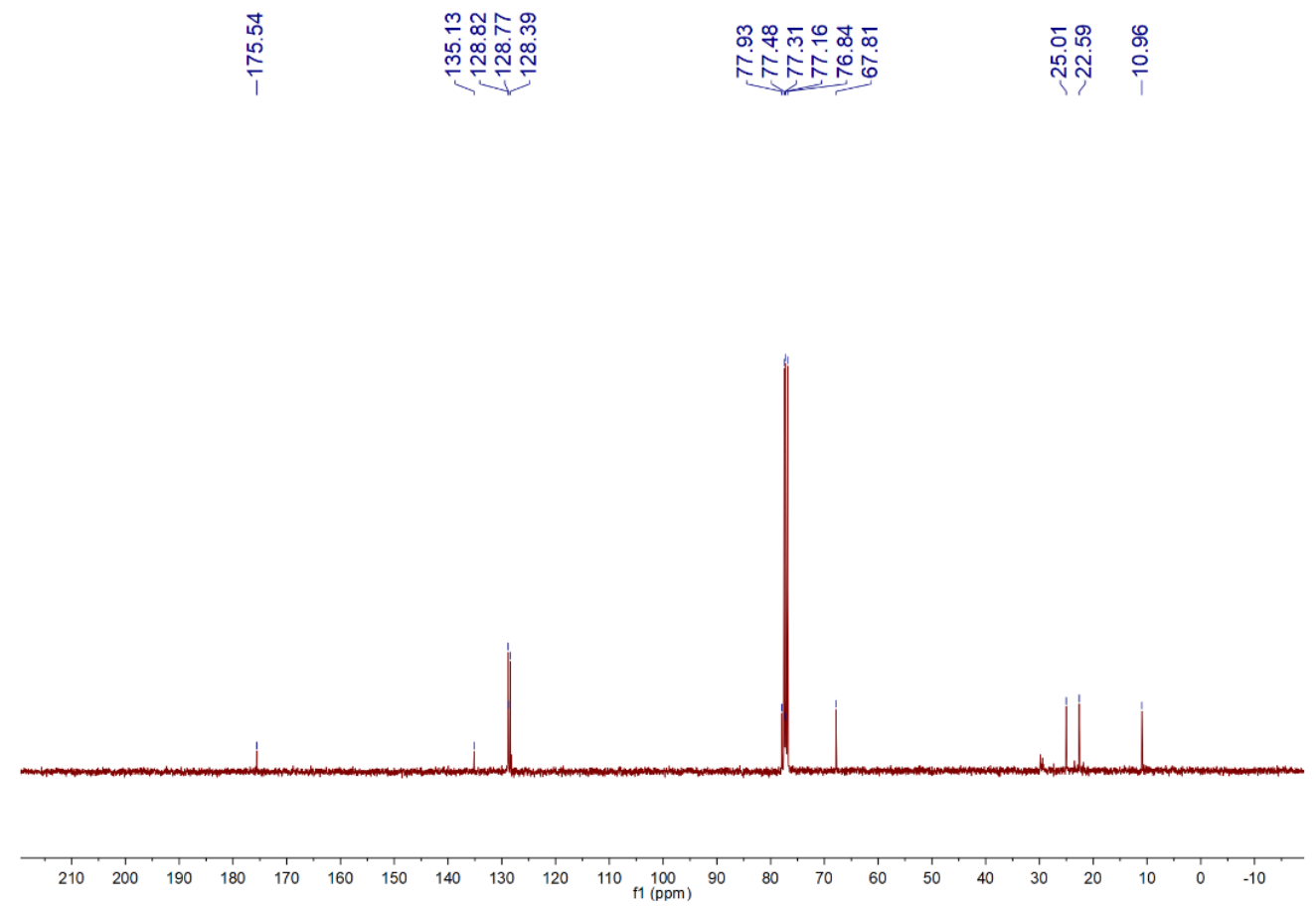

Figure S98. ${ }^{13} \mathrm{C}$ NMR $\left(100 \mathrm{MHz}, \mathrm{CDCl}_{3}\right)$ of $\mathbf{8 a}$. 


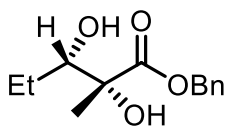

$8 b$

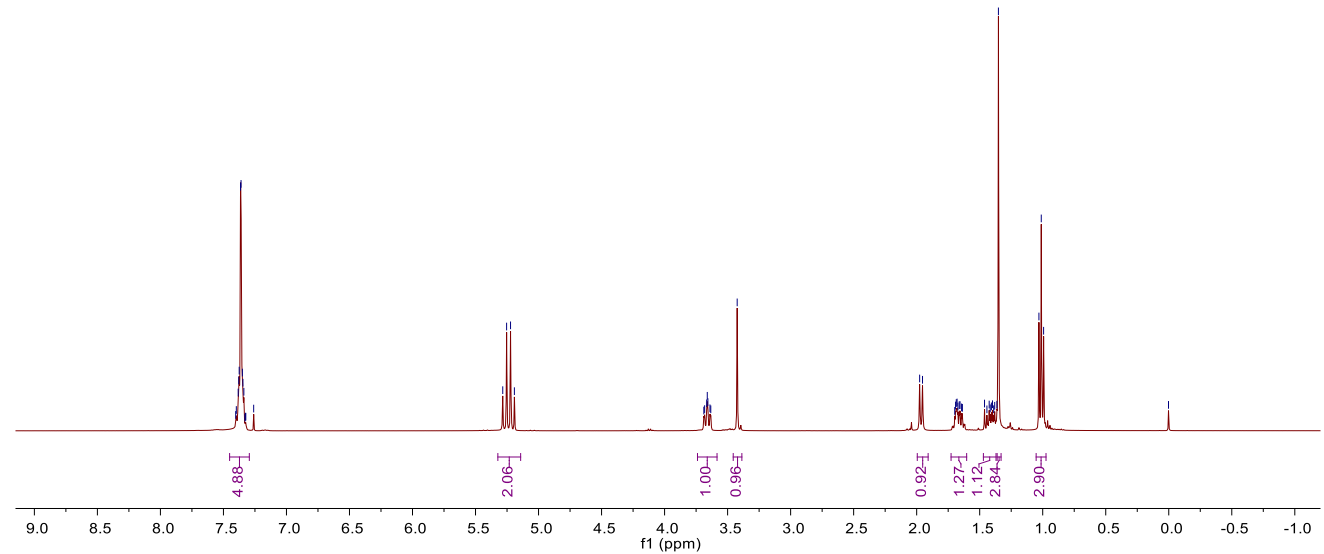

Figure S99. ${ }^{1} \mathrm{H}$ NMR $\left(400 \mathrm{MHz}, \mathrm{CDCl}_{3}\right)$ of $\mathbf{8 b}$.

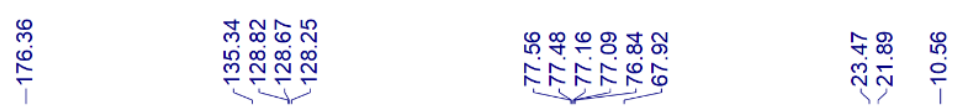

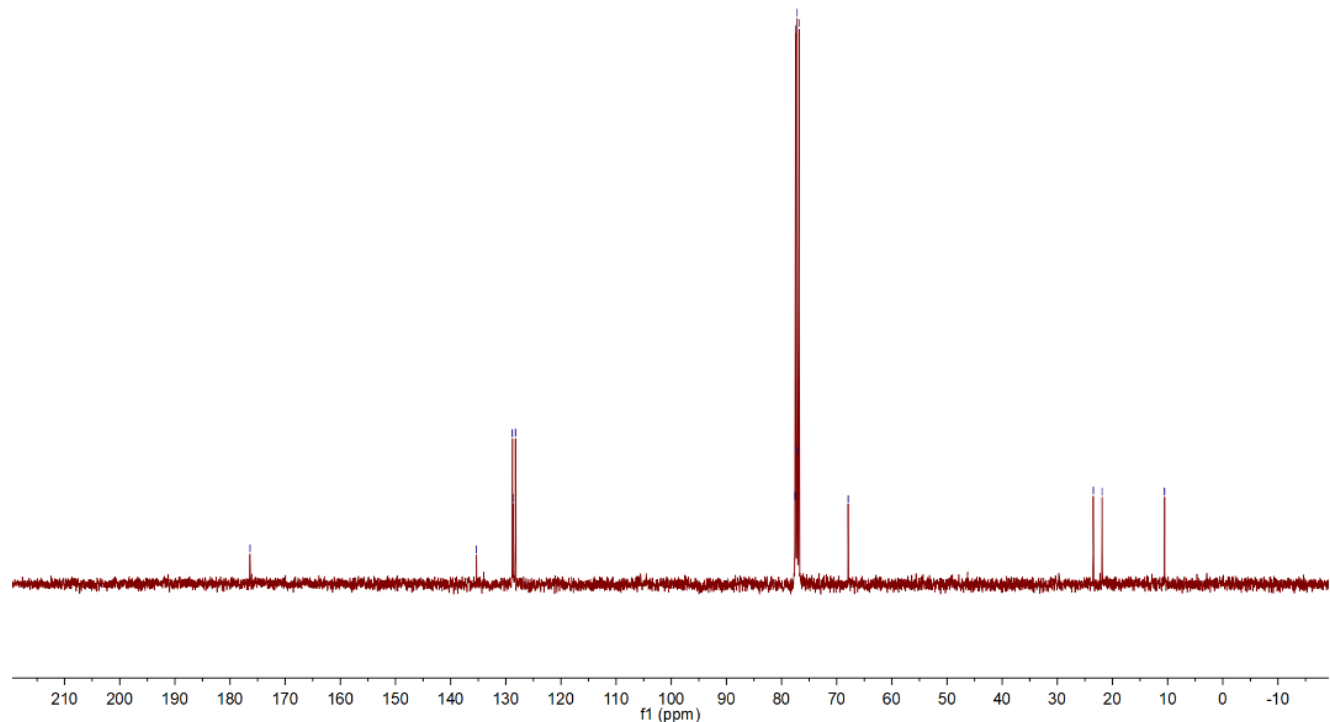

Figure S100. ${ }^{13} \mathrm{C}$ NMR $\left(100 \mathrm{MHz}, \mathrm{CDCl}_{3}\right)$ of $\mathbf{8 b}$. 


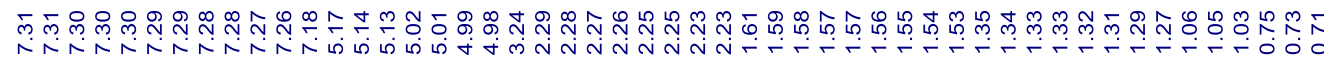<smiles>C[C@H](OC(=O)CO[Na])[C@@H](O)C(=O)Cc1ccccc1</smiles>
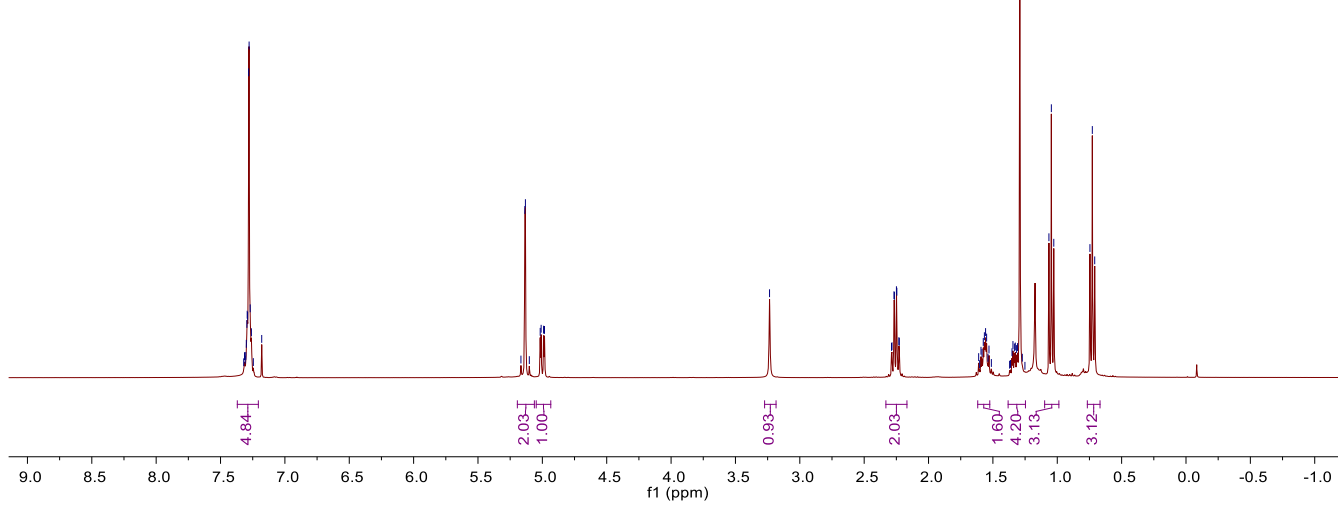

Figure S101. ${ }^{1} \mathrm{H} \mathrm{NMR}\left(400 \mathrm{MHz}, \mathrm{CDCl}_{3}\right)$ of 9 a.

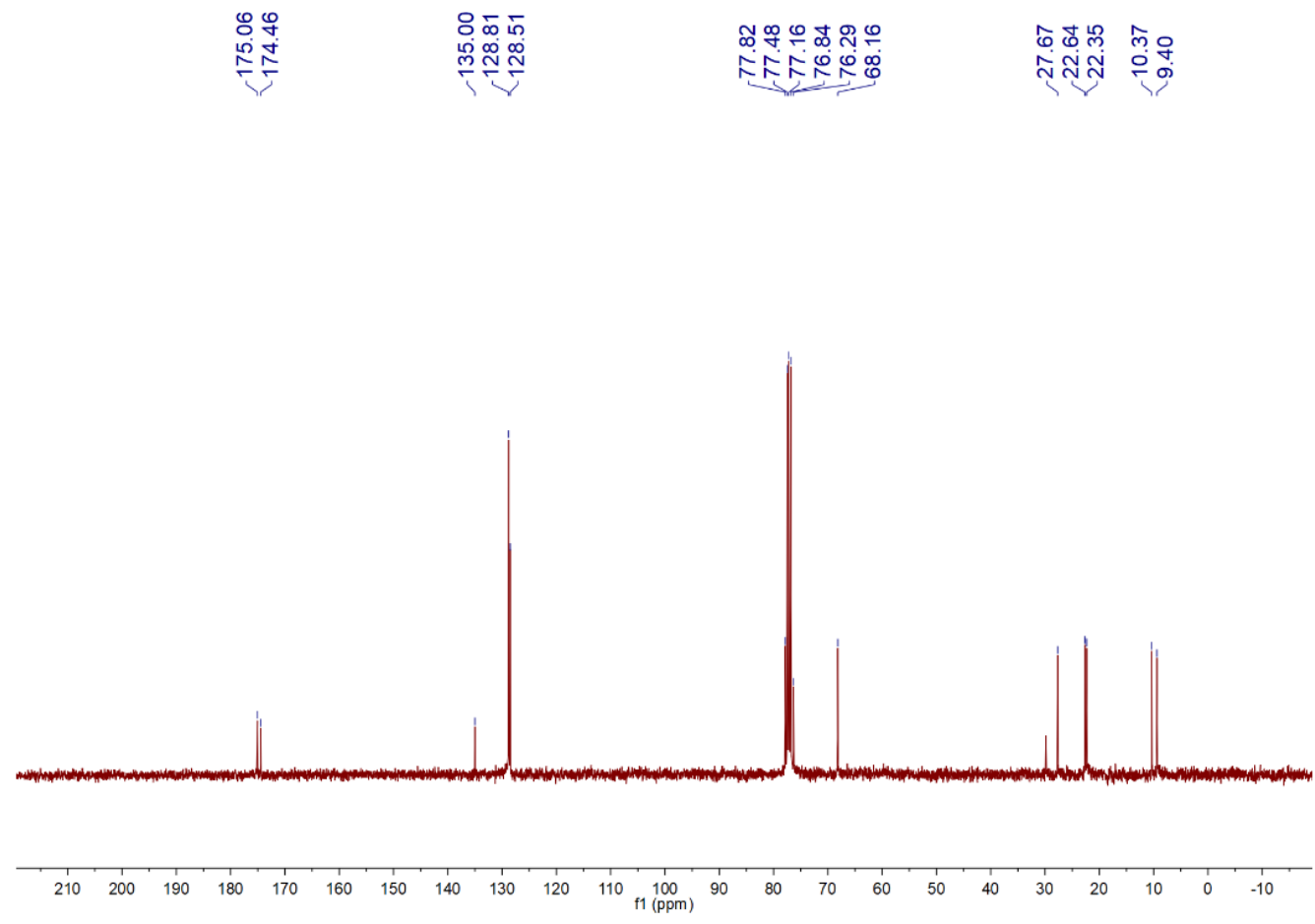

Figure S102. ${ }^{13} \mathrm{C}$ NMR $\left(100 \mathrm{MHz}, \mathrm{CDCl}_{3}\right)$ of $9 \mathbf{a}$. 


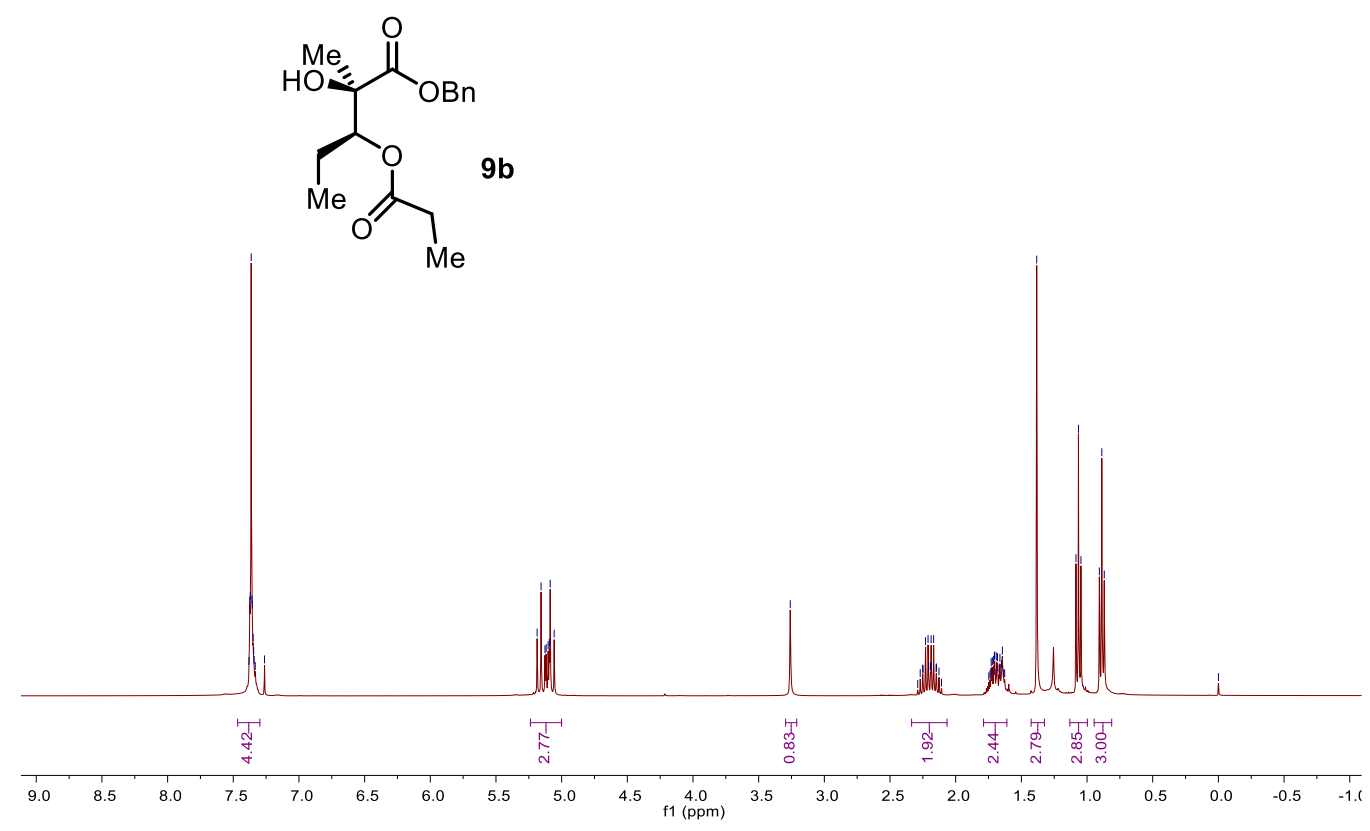

Figure S103. ${ }^{1} \mathrm{H}$ NMR $\left(400 \mathrm{MHz}, \mathrm{CDCl}_{3}\right)$ of $\mathbf{9 b}$.

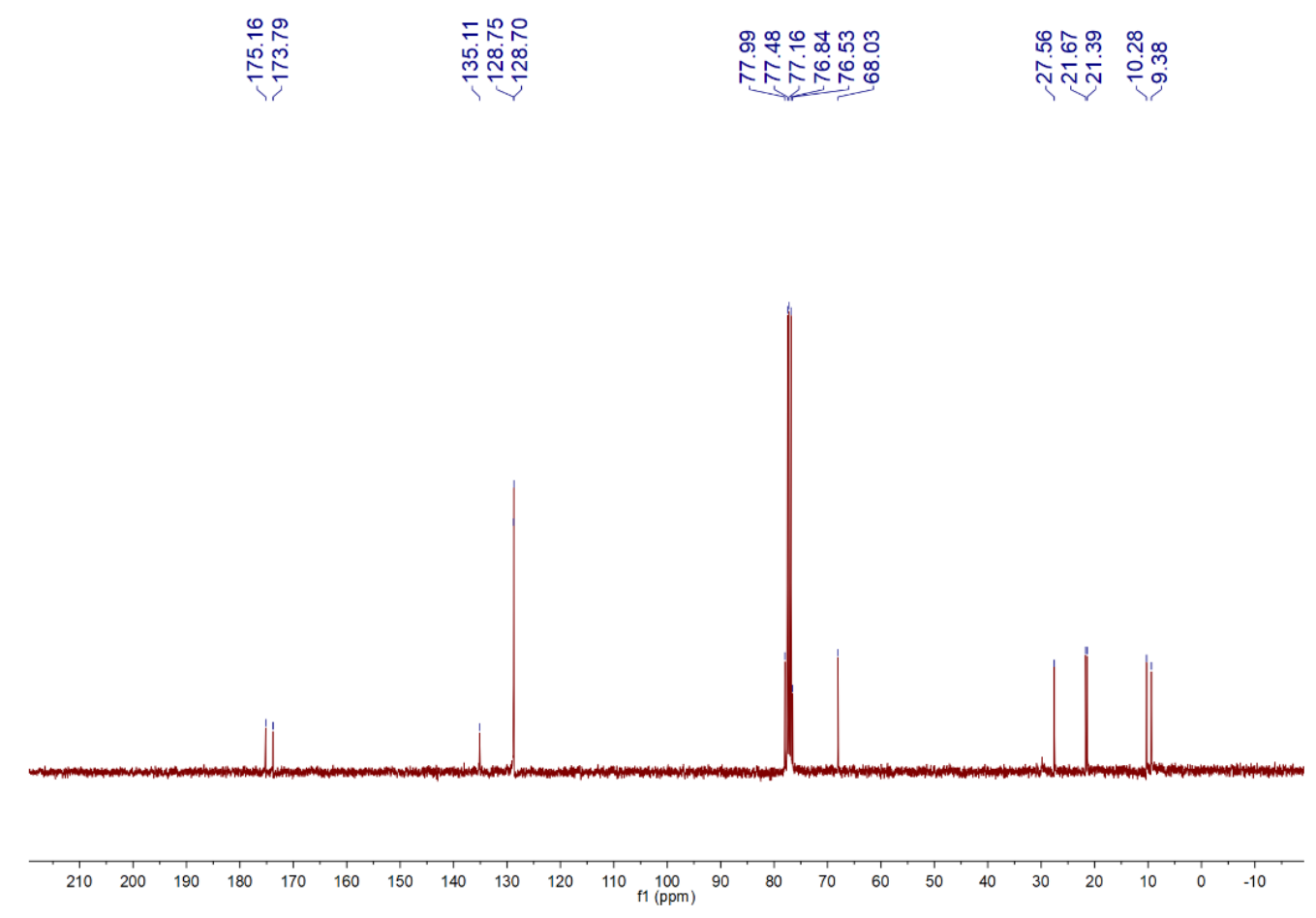

Figure S104. ${ }^{13} \mathrm{C}$ NMR $\left(100 \mathrm{MHz}, \mathrm{CDCl}_{3}\right)$ of $9 \mathbf{b}$. 


\begin{tabular}{|c|c|c|c|}
\hline 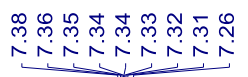 & 离 & 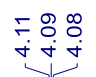 & 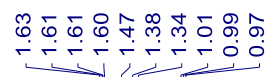 \\
\hline
\end{tabular}
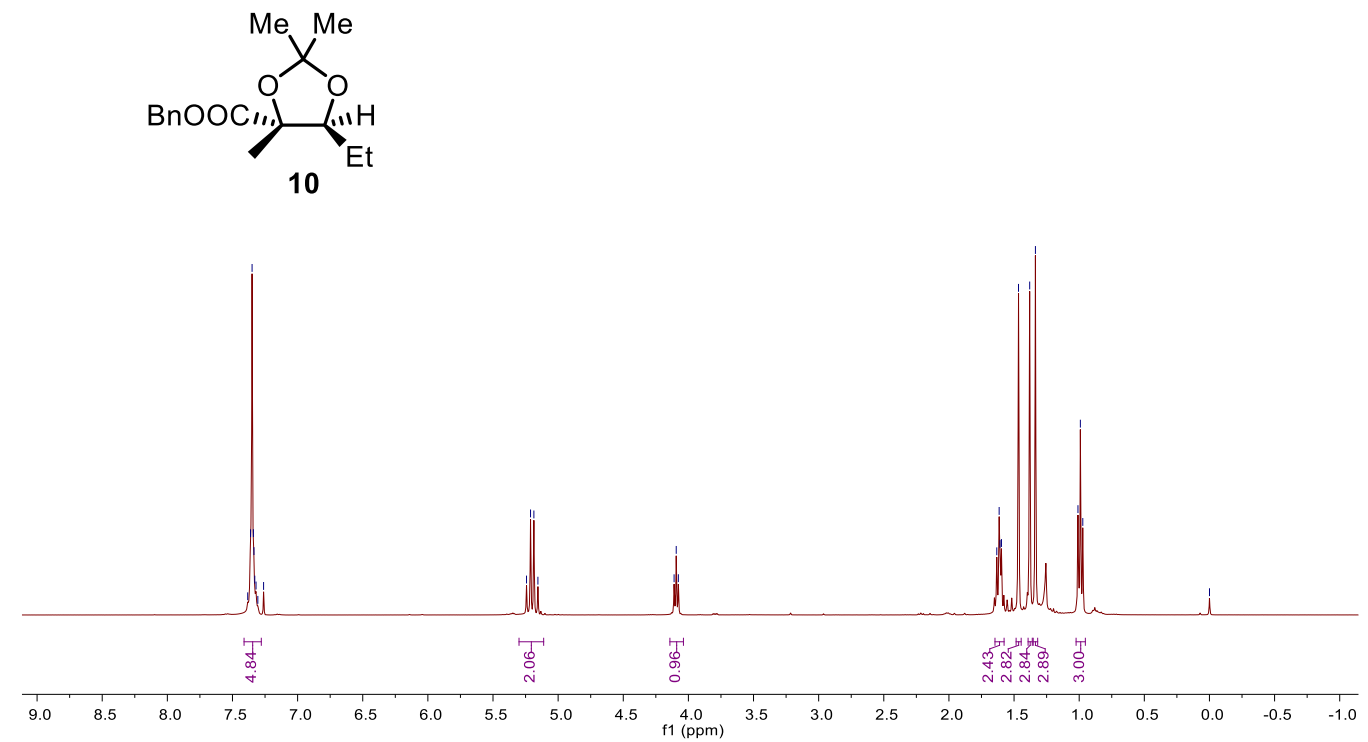

Figure S105. ${ }^{1} \mathrm{H} \mathrm{NMR}\left(400 \mathrm{MHz}, \mathrm{CDCl}_{3}\right)$ of $\mathbf{1 0}$.

\begin{tabular}{|c|c|c|}
\hline 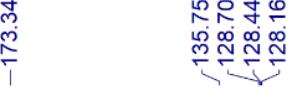 & $\begin{array}{l}\overline{0} \\
\stackrel{\circ}{\circ}\end{array}$ & 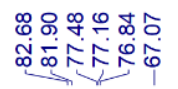 \\
\hline
\end{tabular}

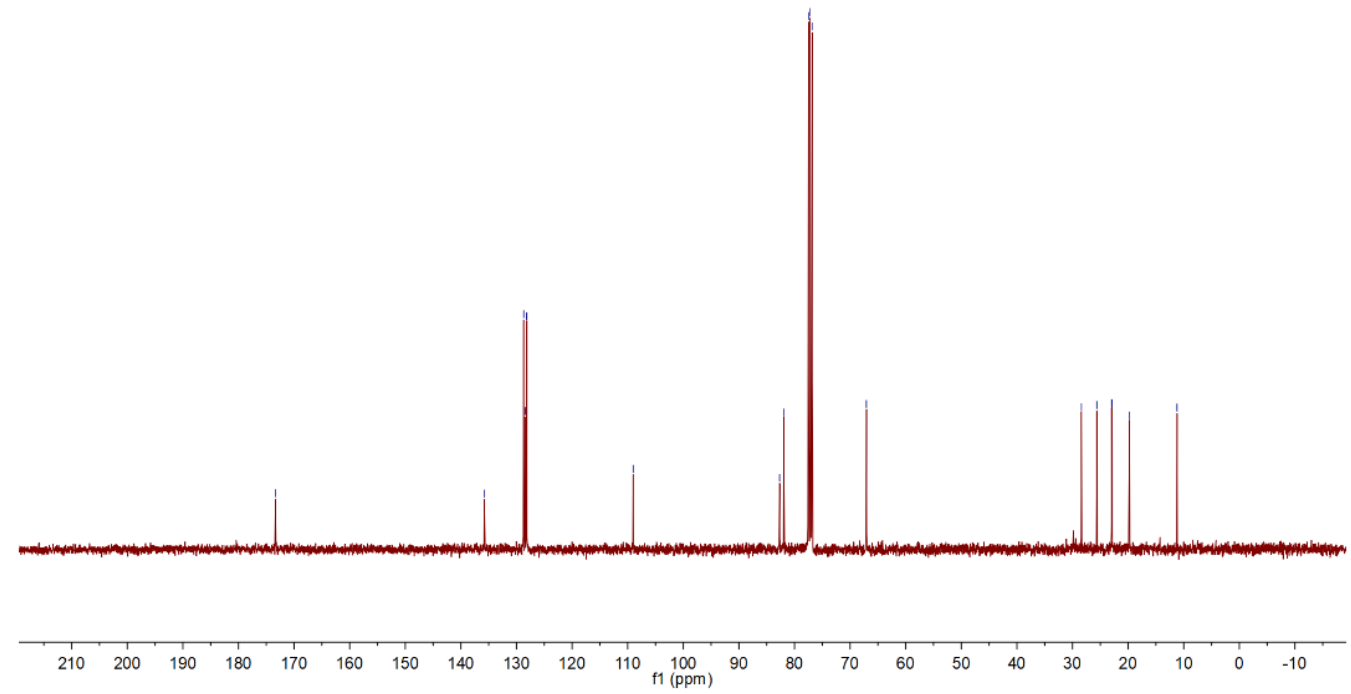

Figure S106. ${ }^{13} \mathrm{C}$ NMR $\left(100 \mathrm{MHz}, \mathrm{CDCl}_{3}\right)$ of $\mathbf{1 0}$. 
2. Copies of HPLC Data

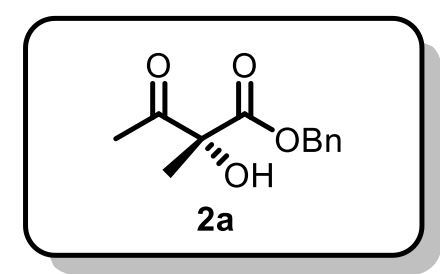

HPLC (ChiralPak As-H*2, 5\% i-PrOH in hexanes, $0.8 \mathrm{~mL} / \mathrm{min}, 206 \mathrm{~nm}$ )

$\langle$ Chromatogram〉

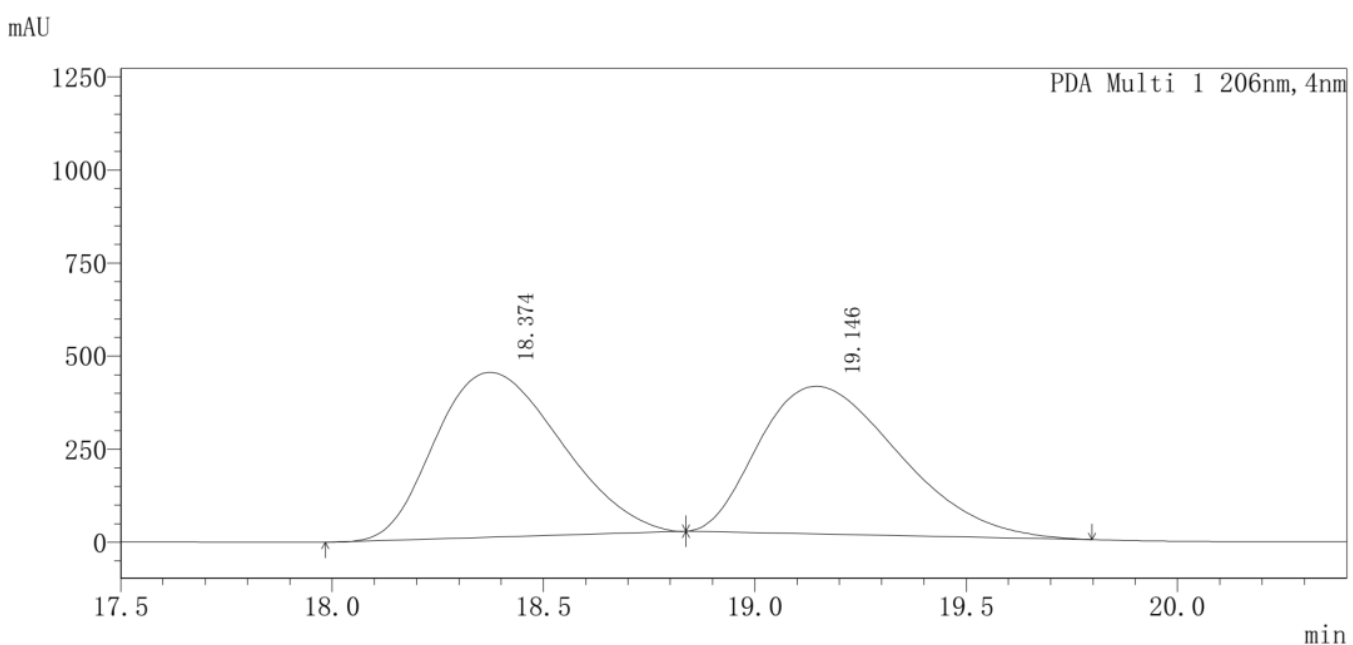

$\langle$ Peak Results $>$

PDA Ch1 206nm

\begin{tabular}{|c|c|c|c|c|}
\hline Index & Time/min & Height/mAU & Quantity/Area & Area \%/\% \\
\hline 1 & 18.374 & 443011 & 9182062 & 50.045 \\
\hline 2 & 19.146 & 396957 & 9165407 & 49.955 \\
\hline
\end{tabular}

$\langle$ Chromatogram〉

mAU

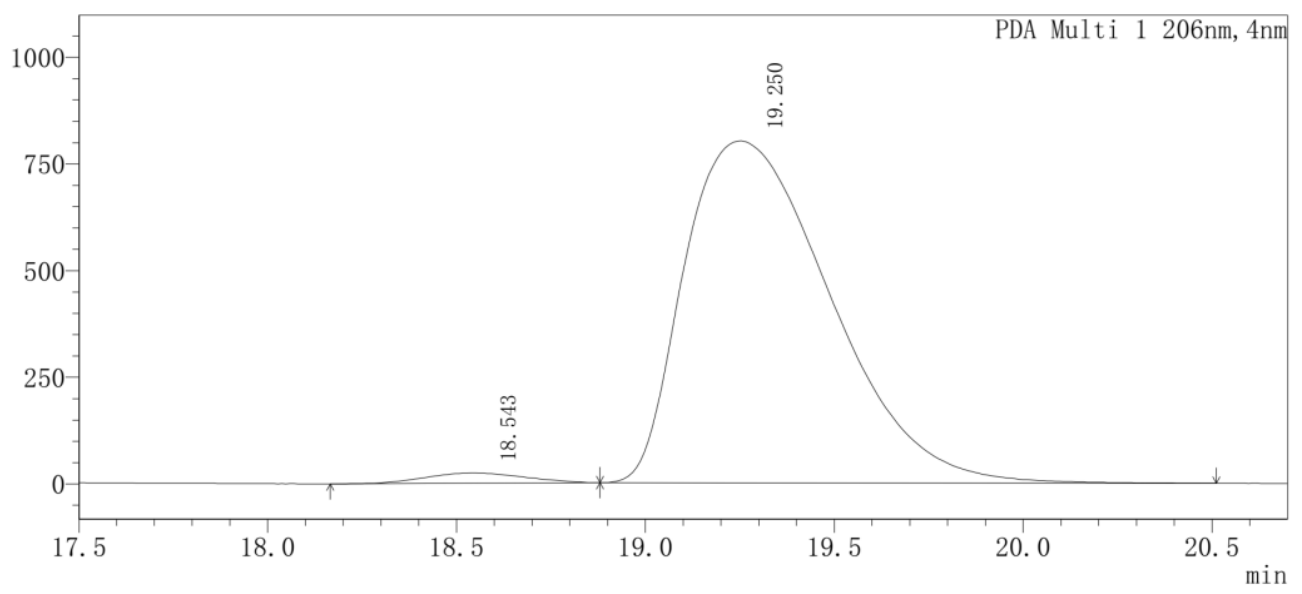

〈Peak Results〉

PDA Ch1 206nm

\begin{tabular}{|c|c|c|c|c|}
\hline Index & Time/min & Height/mAU & Quantity/Area & Area \%/\% \\
\hline 1 & 18.543 & 23929 & 441753 & 2.007 \\
\hline 2 & 19.250 & 801621 & 21569362 & 97.993 \\
\hline
\end{tabular}




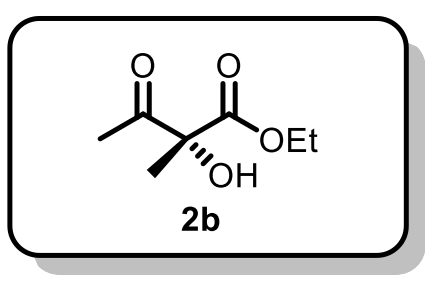

HPLC (ChiralPak OJ-H, 10\% i-PrOH in hexanes, $1.0 \mathrm{~mL} / \mathrm{min}, 230 \mathrm{~nm}$ )

$\langle$ Chromatogram〉

$\mathrm{mAU}$

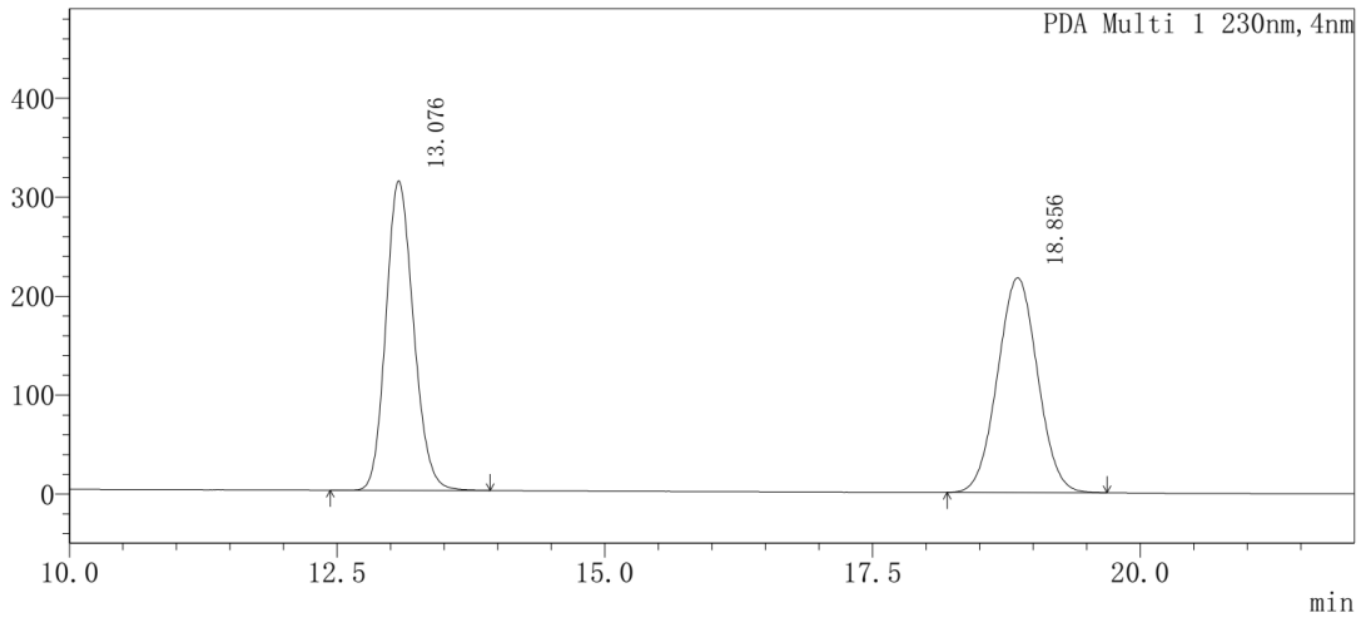

〈Peak Results〉

PDA Ch1 230nm

\begin{tabular}{|c|c|c|c|c|}
\hline Index & Time/min & Height/mAU & Quantity/Area & Area \%/\% \\
\hline 1 & 13.076 & 312864 & 5610882 & 49.815 \\
\hline 2 & 18.856 & 217038 & 5652533 & 50.185 \\
\hline
\end{tabular}

$\langle$ Chromatogram〉

$\mathrm{mAU}$

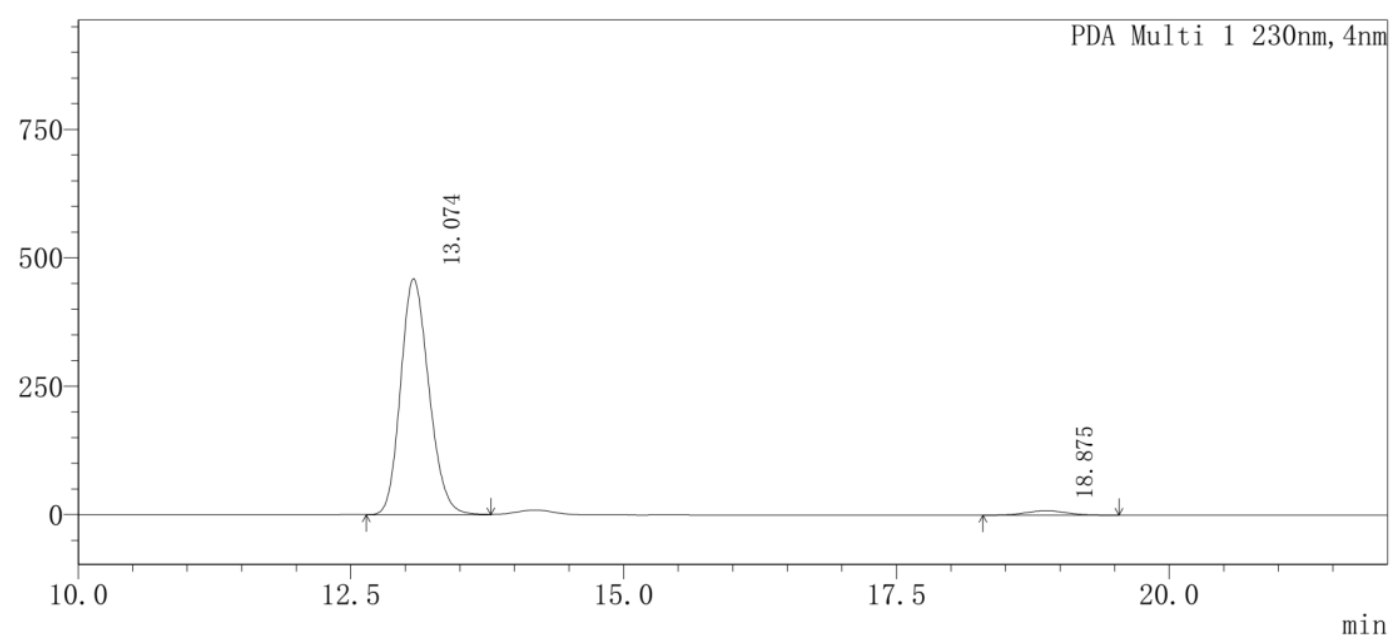

$\langle$ Peak Results〉

PDA Ch1 230nm

\begin{tabular}{|c|c|c|c|c|}
\hline Index & Time/min & Height/mAU & Quantity/Area & Area \%/\% \\
\hline 1 & 13.074 & 460110 & 8324349 & 97.401 \\
\hline 2 & 18.875 & 8545 & 222145 & 2.599 \\
\hline
\end{tabular}




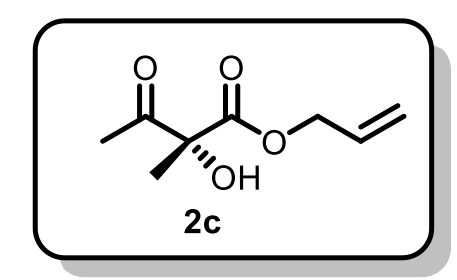

HPLC (ChiralPak OJ-H, 10\% i-PrOH in hexanes, $1.0 \mathrm{~mL} / \mathrm{min}, 230 \mathrm{~nm}$ )

〈Chromatogram〉

mAU

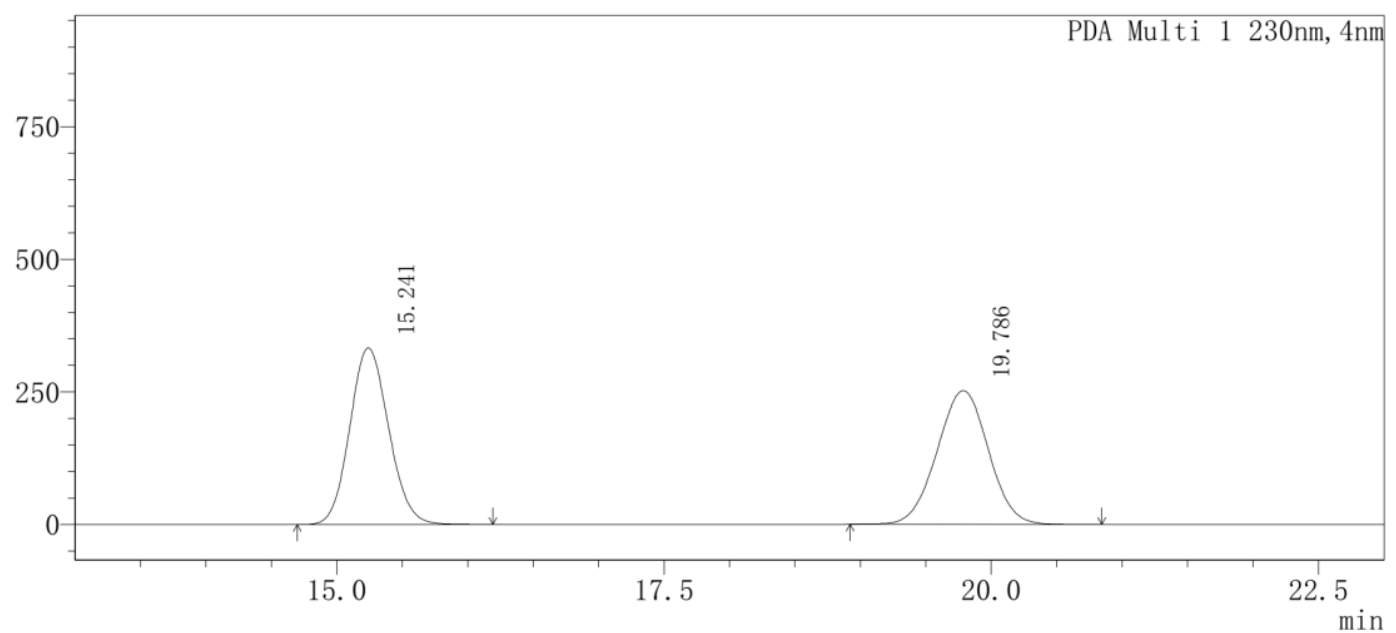

〈Peak Results〉

PDA Ch1 230nm

\begin{tabular}{|c|c|c|c|c|}
\hline Index & Time/min & Height/mAU & Quantity/Area & Area \%/\% \\
\hline 1 & 15.241 & 333256 & 6885620 & 50.022 \\
\hline 2 & 19.786 & 252250 & 6879679 & 49.978 \\
\hline
\end{tabular}

〈Chromatogram〉

$\mathrm{mAU}$

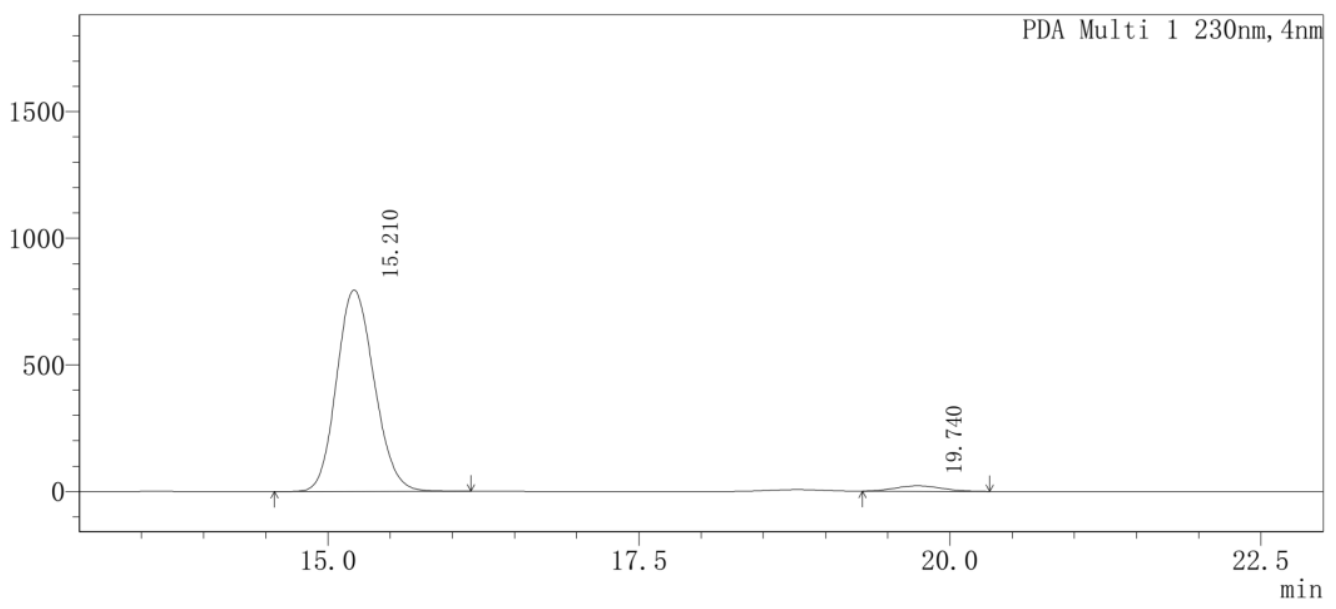

〈Peak Results〉

PDA Ch1 230nm

\begin{tabular}{|c|c|c|c|c|} 
PDA Ch1 $230 \mathrm{~nm}$ & & & \\
\hline Index & Time/min & Height/mAU & Quantity/Area & Area $\% /$ \\
\hline 1 & 15.210 & 795604 & 16751437 & 96.826 \\
\hline 2 & 19.740 & 21400 & 549136 & 3.174 \\
\hline
\end{tabular}




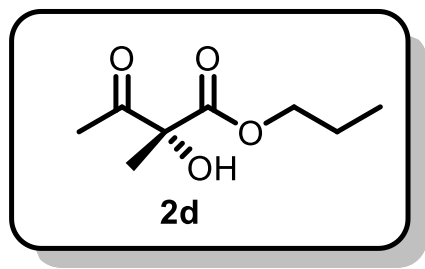

HPLC (ChiralPak OJ-H, 10\% i-PrOH in hexanes, $1.0 \mathrm{~mL} / \mathrm{min}, 230 \mathrm{~nm}$ )

〈Chromatogram〉

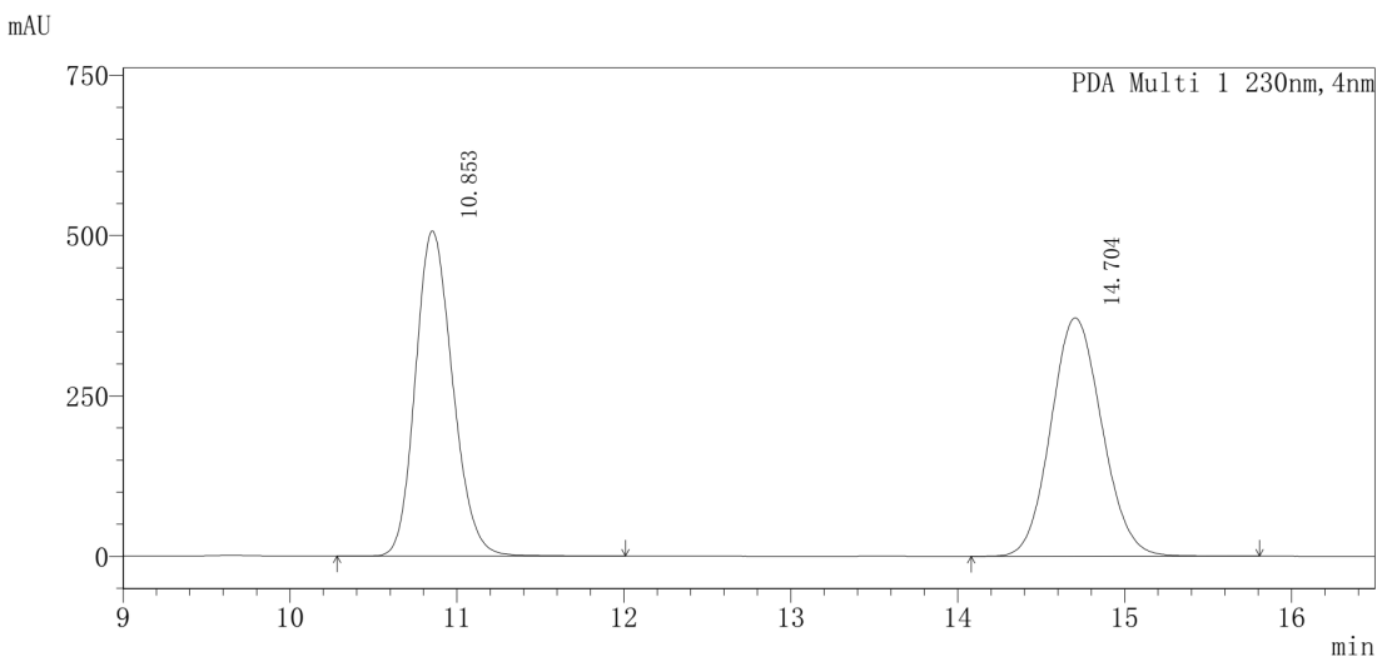

〈Peak Results〉

PDA Ch1 230nm

\begin{tabular}{|c|c|c|c|c|}
\hline Index & Time/min & Height/mAU & Quantity/Area & Area \%/\% \\
\hline 1 & 10.853 & 507144 & 7876937 & 49.885 \\
\hline 2 & 14.704 & 371883 & 7913118 & 50.115 \\
\hline
\end{tabular}

〈Chromatogram〉

$\mathrm{mAU}$

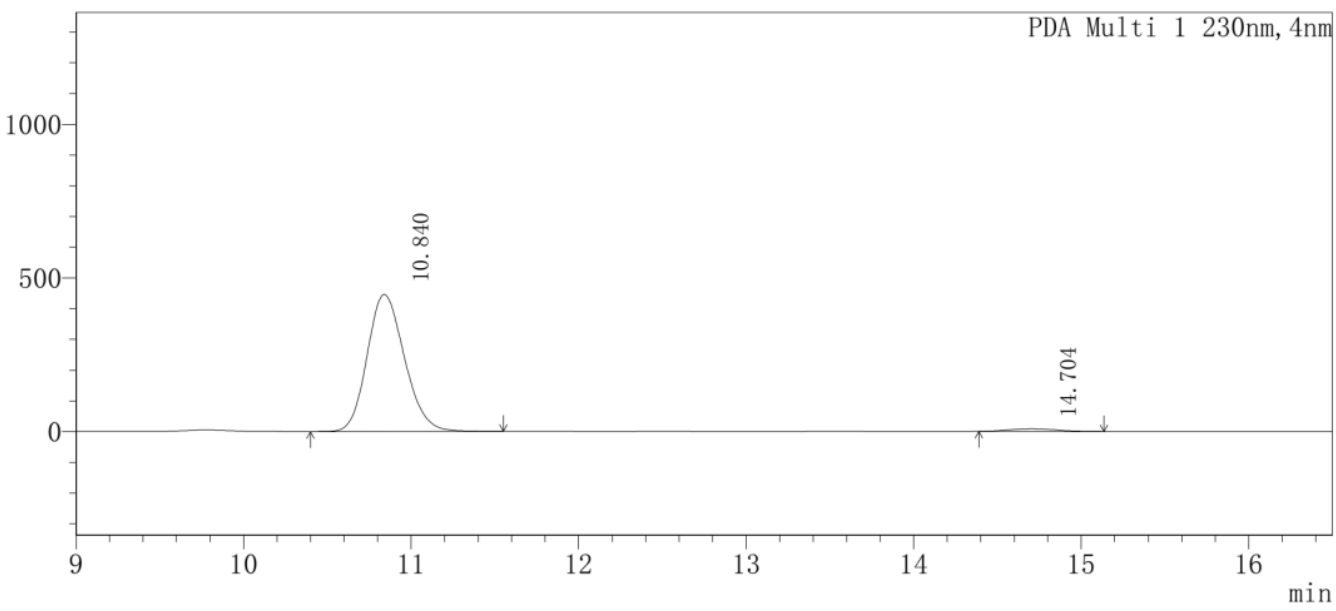

〈Peak Results〉

PDA Ch1 230nm

\begin{tabular}{|c|c|c|c|c|}
\hline Index & Time/min & Height/mAU & Quantity/Area & Area \%/\% \\
\hline 1 & 10.840 & 446520 & 6911813 & 97.533 \\
\hline 2 & 14.704 & 8870 & 174857 & 2.467 \\
\hline
\end{tabular}




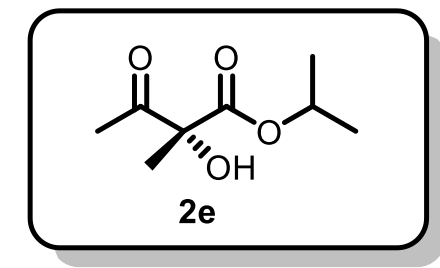

HPLC (ChiralPak OJ-H, 10\% i-PrOH in hexanes, $1.0 \mathrm{~mL} / \mathrm{min}, 230 \mathrm{~nm}$ )

$\langle$ Chromatogram〉

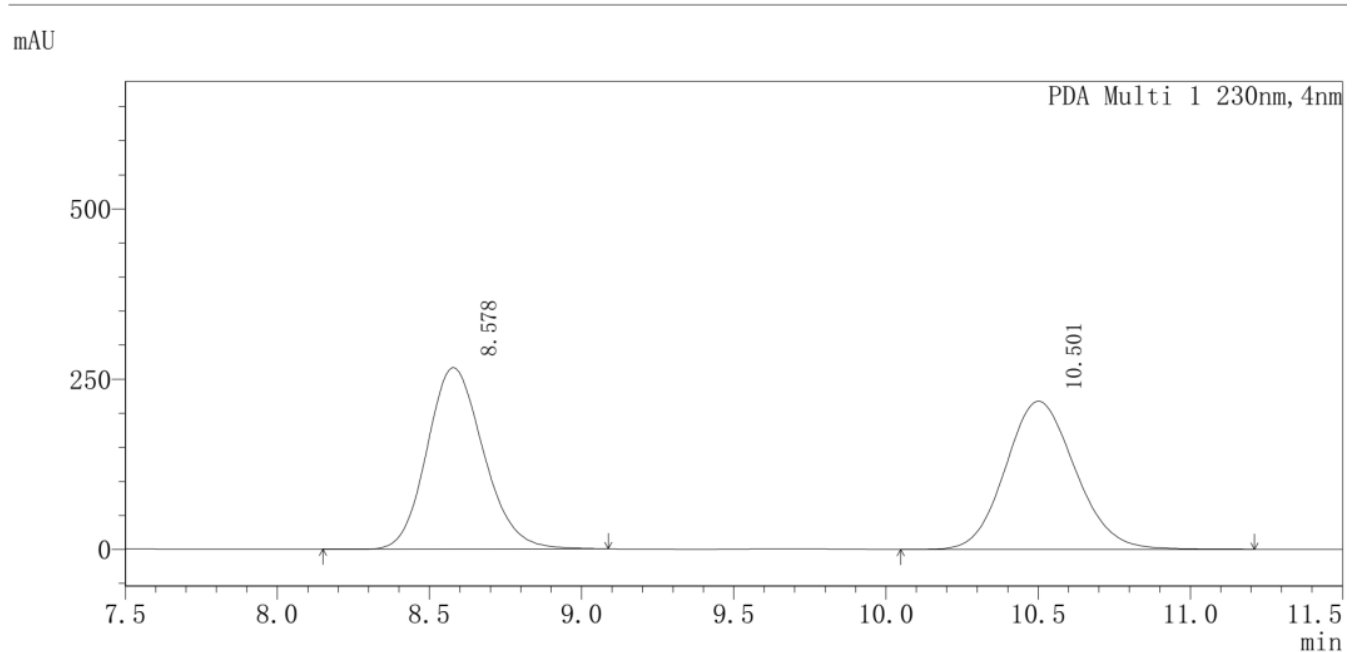

〈Peak Results〉

PDA Ch1 230nm

\begin{tabular}{|c|c|c|c|c|}
\hline Index & Time/min & Height/mAU & Quantity/Area & Area \%/\% \\
\hline 1 & 8.578 & 266518 & 3437067 & 49.837 \\
\hline 2 & 10.501 & 217381 & 3459534 & 50.163 \\
\hline
\end{tabular}

$\langle$ Chromatogram〉

$\mathrm{mAU}$

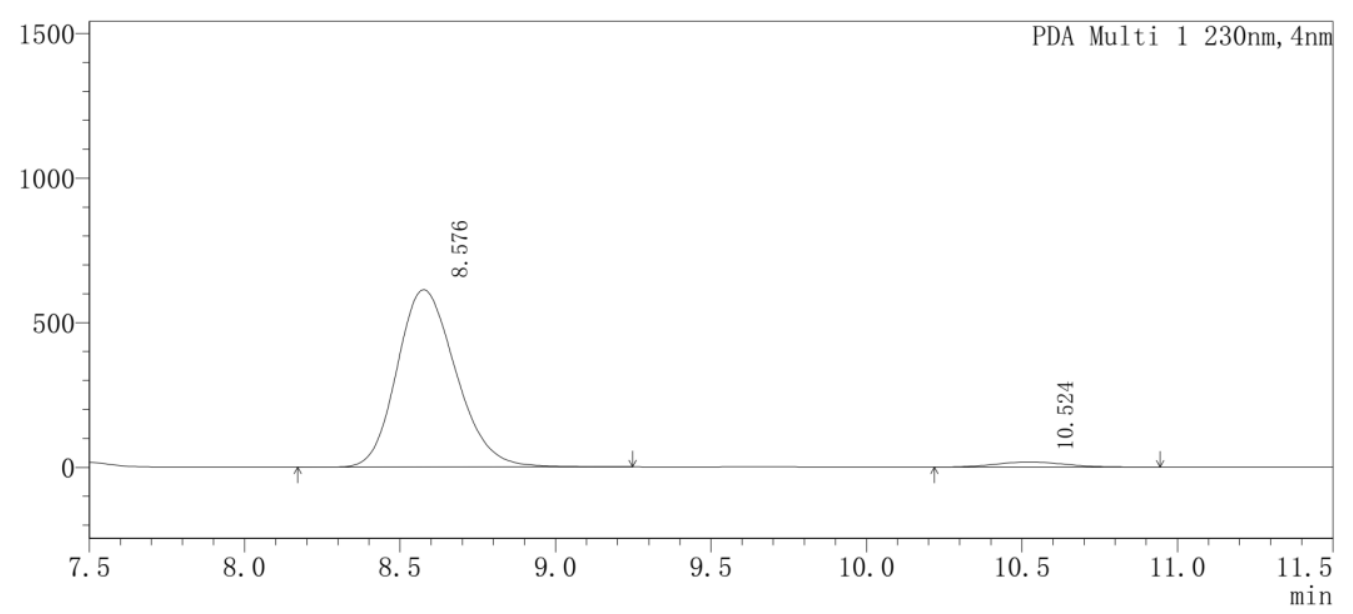

〈Peak Results〉

PDA Ch1 230nm

\begin{tabular}{|c|c|c|c|c|}
\hline Index & Time/min & Height/mAU & Quantity/Area & Area \%/\% \\
\hline 1 & 8.576 & 614469 & 8086445 & 96.667 \\
\hline 2 & 10.524 & 17811 & 278826 & 3.333 \\
\hline
\end{tabular}




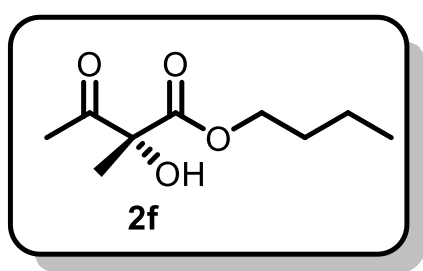

HPLC (ChiralPak OJ-H, 10\% i-PrOH in hexanes, $1.0 \mathrm{~mL} / \mathrm{min}, 230 \mathrm{~nm}$ )

〈Chromatogram〉

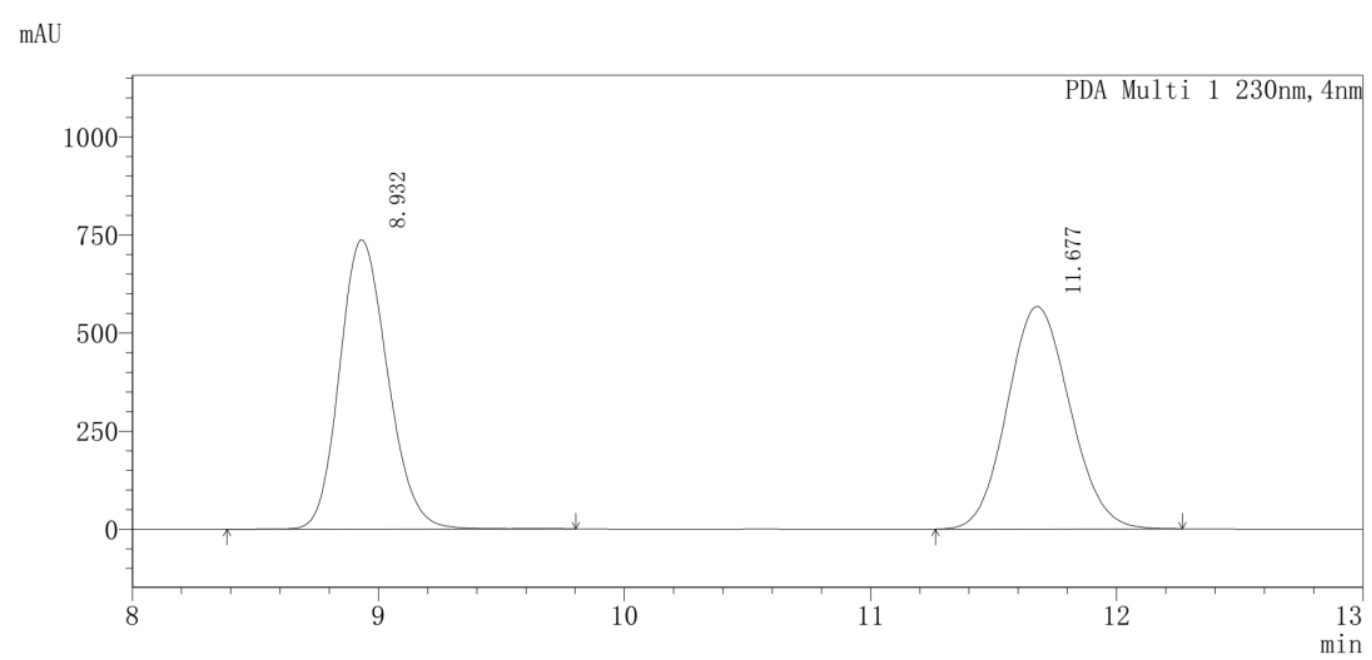

〈Peak Results〉

PDA Ch1 230nm

\begin{tabular}{|c|c|c|c|c|}
\hline Index & Time/min & Height/mAU & Quantity/Area & Area \%/\% \\
\hline 1 & 8.932 & 737275 & 9965663 & 49.818 \\
\hline 2 & 11.677 & 567235 & 10038582 & 50.182 \\
\hline
\end{tabular}

\section{〈Chromatogram〉}

$\mathrm{mAU}$

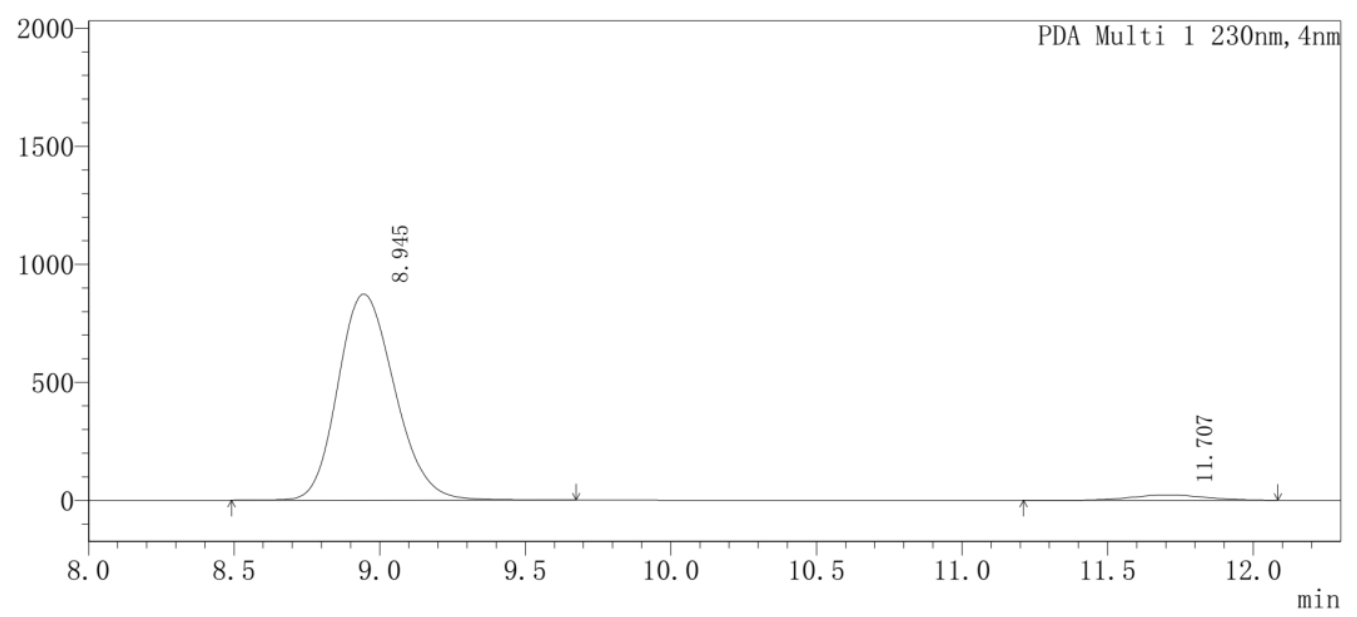

〈Peak Results〉

PDA Ch1 230nm

\begin{tabular}{|c|c|c|c|c|}
\hline Index & Time/min & Height/mAU & Quantity/Area & Area \%/\% \\
\hline 1 & 8.945 & 872643 & 11803805 & 96.844 \\
\hline 2 & 11.707 & 22316 & 384675 & 3.156 \\
\hline
\end{tabular}




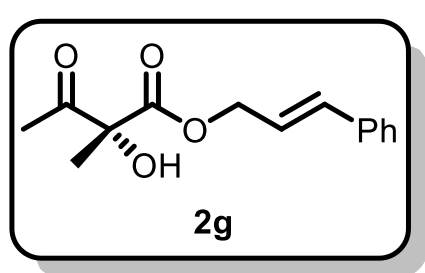

HPLC (ChiralPak AD-H, 5\% i-PrOH in hexanes, $1.0 \mathrm{~mL} / \mathrm{min}, 250 \mathrm{~nm}$ )

〈Chromatogram〉

$\mathrm{mAU}$

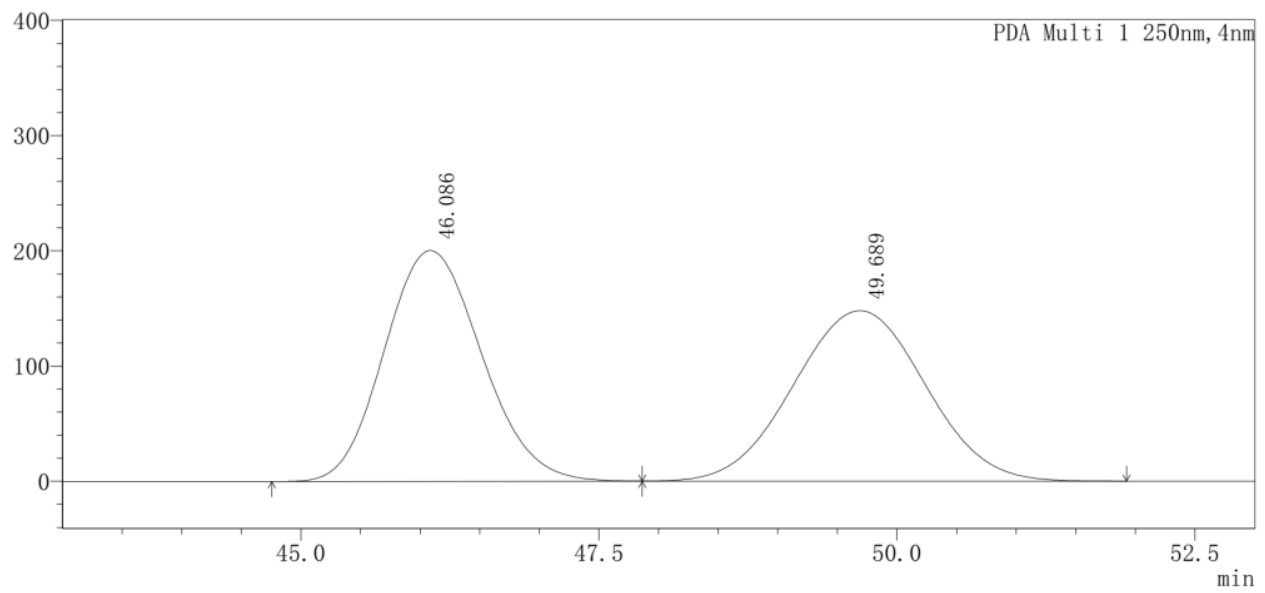

〈Peak Results〉

PDA Ch1 250nm

\begin{tabular}{|c|c|c|c|c|}
\hline Index & Time/min & Height/mAU & Quantity/Area & Area \%/\% \\
\hline 1 & 46.086 & 200391 & 11394835 & 49.944 \\
\hline 2 & 49.689 & 147989 & 11420407 & 50.056 \\
\hline
\end{tabular}

〈Chromatogram〉

$\mathrm{mAU}$

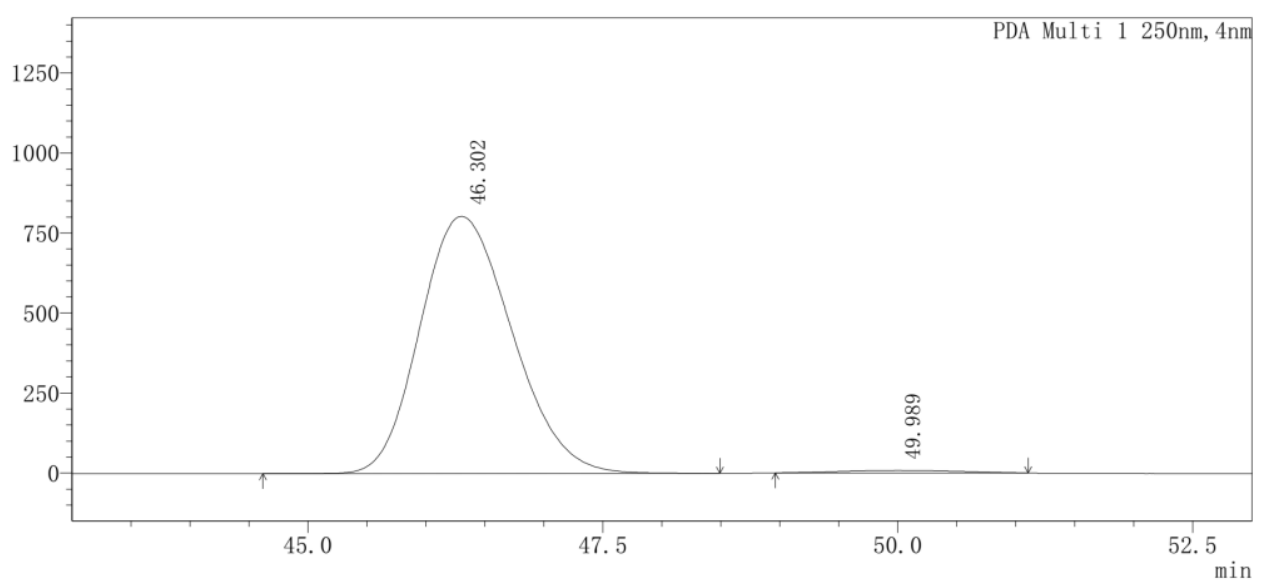

〈Peak Results〉

PDA Ch1 250nm

\begin{tabular}{|c|c|c|c|c|}
\hline Index & Time/min & Height/mAU & Quantity/Area & Area \%/\% \\
\hline 1 & 46.302 & 803242 & 43525497 & 98.757 \\
\hline 2 & 49.989 & 7557 & 548043 & 1.243 \\
\hline
\end{tabular}




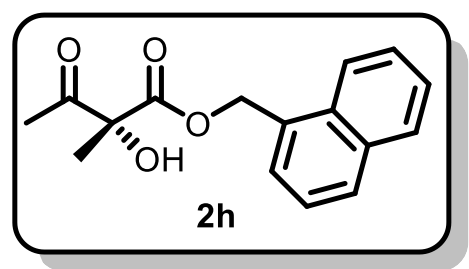

HPLC (ChiralPak AS-H*2, 5\% i-PrOH in hexanes, $0.8 \mathrm{~mL} / \mathrm{min}, 223 \mathrm{~nm}$ )

〈Chromatogram〉

$\mathrm{mAU}$

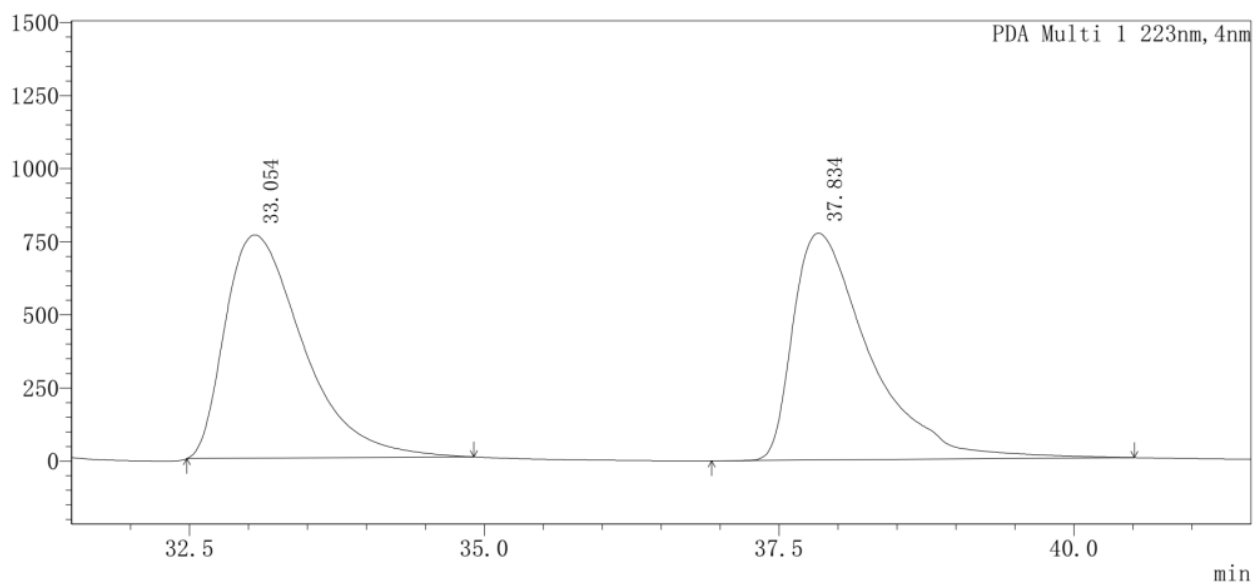

〈Peak Results〉

PDA Ch1 223nm

\begin{tabular}{|c|c|c|c|c|}
\hline Index & Time/min & Height/mAU & Quantity/Area & Area \%/\% \\
\hline 1 & 33.054 & 763545 & 35220769 & 50.117 \\
\hline 2 & 37.834 & 775569 & 35055936 & 49.883 \\
\hline
\end{tabular}

〈Chromatogram〉

$\mathrm{mAU}$

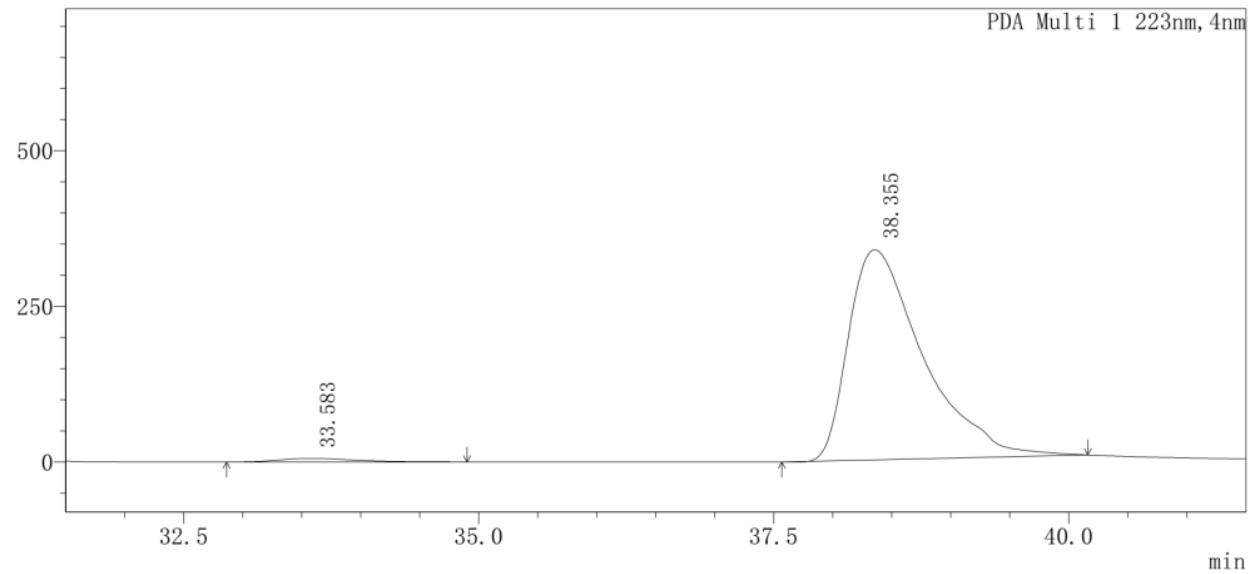

〈Peak Results〉

PDA Ch1 223nm

\begin{tabular}{|c|c|c|c|c|}
\hline Index & Time/min & Height/mAU & Quantity/Area & Area \%/\% \\
\hline 1 & 33.583 & 5828 & 258131 & 1.703 \\
\hline 2 & 38.355 & 337864 & 14895566 & 98.297 \\
\hline
\end{tabular}




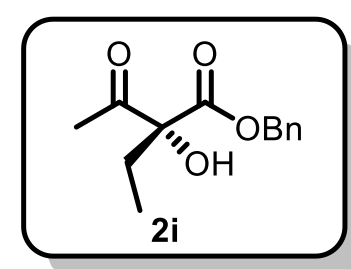

HPLC (ChiralPak AS-H, 2\% i-PrOH in hexanes, $1.0 \mathrm{~mL} / \mathrm{min}, 208 \mathrm{~nm}$ )

$\langle$ Chromatogram〉

$\mathrm{mAU}$

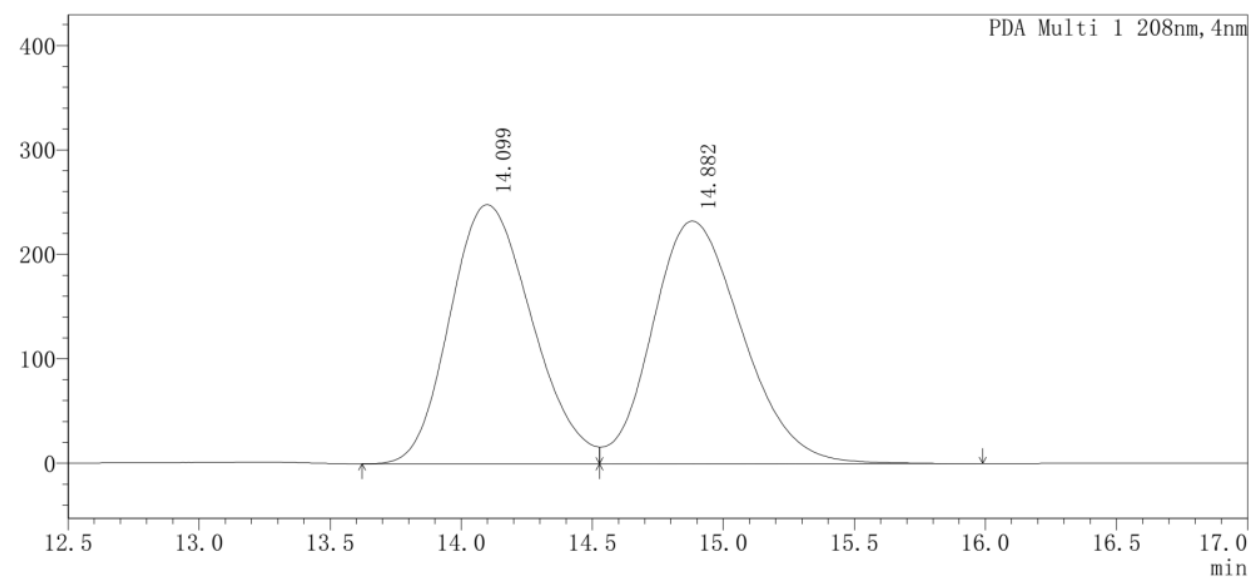

〈Peak Results〉

PDA Ch1 208nm

\begin{tabular}{|c|c|c|c|c|}
\hline Index & Time/min & Height/mAU & Quantity/Area & Area \%/\% \\
\hline 1 & 14.099 & 248250 & 5419377 & 49.433 \\
\hline 2 & 14.882 & 232440 & 5543793 & 50.567 \\
\hline
\end{tabular}

〈Chromatogram〉

$\mathrm{mAU}$

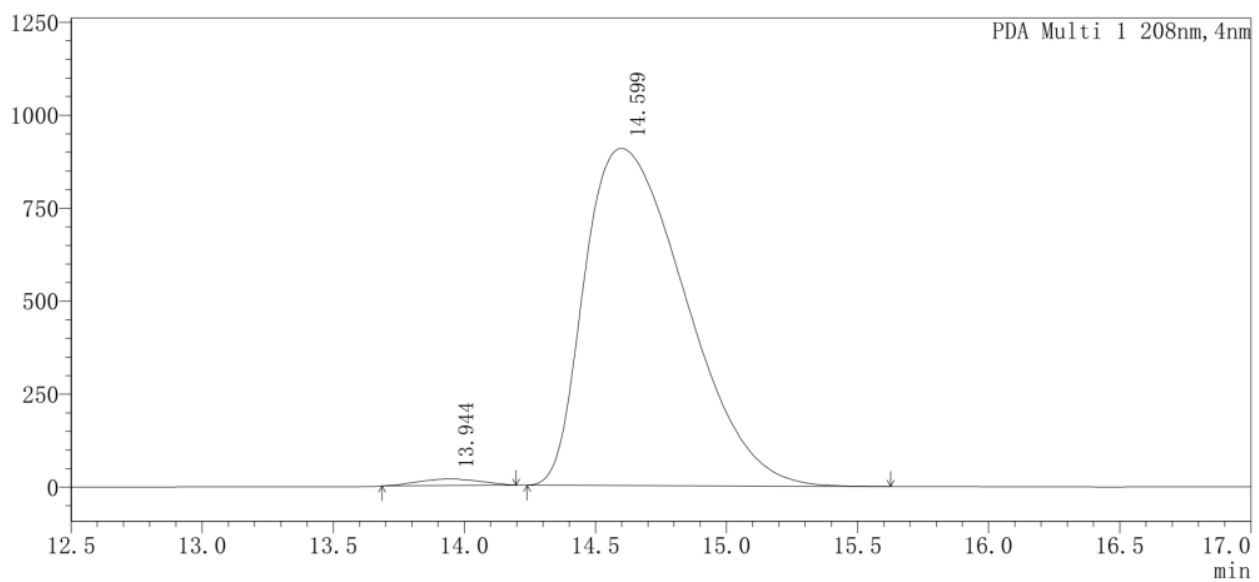

〈Peak Results〉

PDA Ch1 208nm

\begin{tabular}{|c|c|c|c|c|}
\hline Index & Time/min & Height/mAU & Quantity/Area & Area \%/\% \\
\hline 1 & 13.944 & 17626 & 292505 & 1.190 \\
\hline 2 & 14.599 & 906485 & 24285505 & 98.810 \\
\hline
\end{tabular}




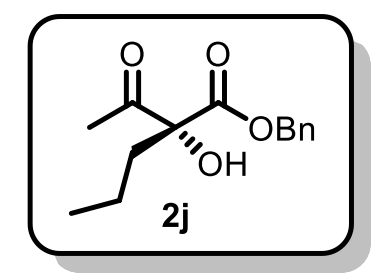

HPLC (ChiralPak AS-H, 2\% i-PrOH in hexanes, $0.8 \mathrm{~mL} / \mathrm{min}, 208 \mathrm{~nm}$ )

$\langle$ Chromatogram〉

$\mathrm{mAU}$

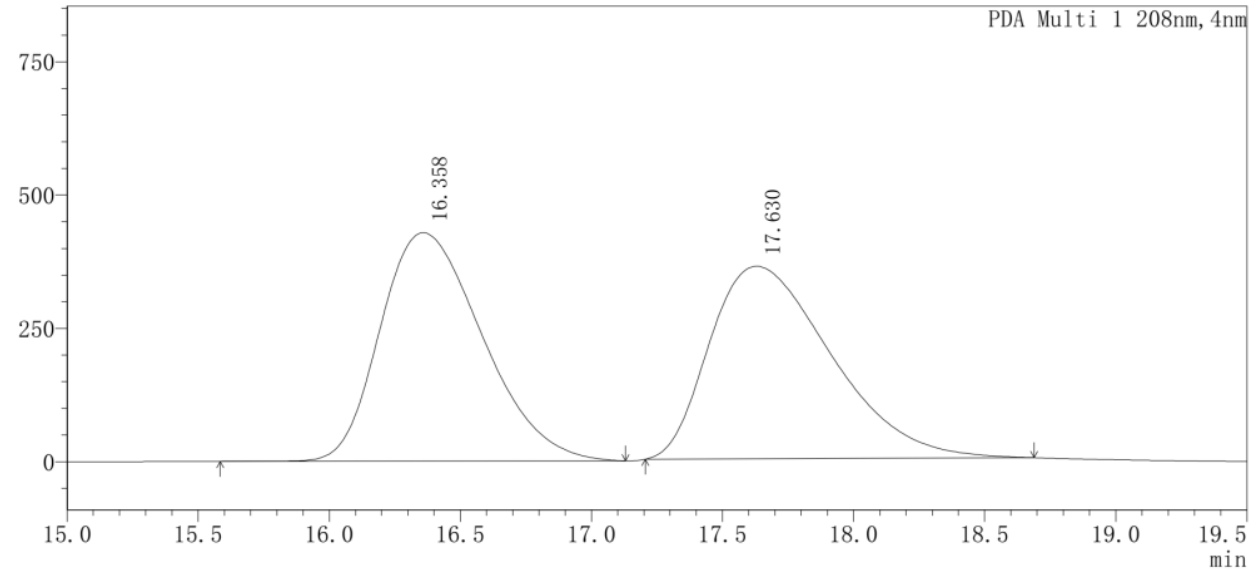

〈Peak Results

PDA Ch1 208nm

\begin{tabular}{|c|c|c|c|c|}
\hline Index & Time/min & Height/mAU & Quantity/Area & Area \%/\% \\
\hline 1 & 16.358 & 428240 & 11605336 & 49.850 \\
\hline 2 & 17.630 & 361300 & 11675275 & 50.150 \\
\hline
\end{tabular}

$\langle$ Chromatogram〉

mAU

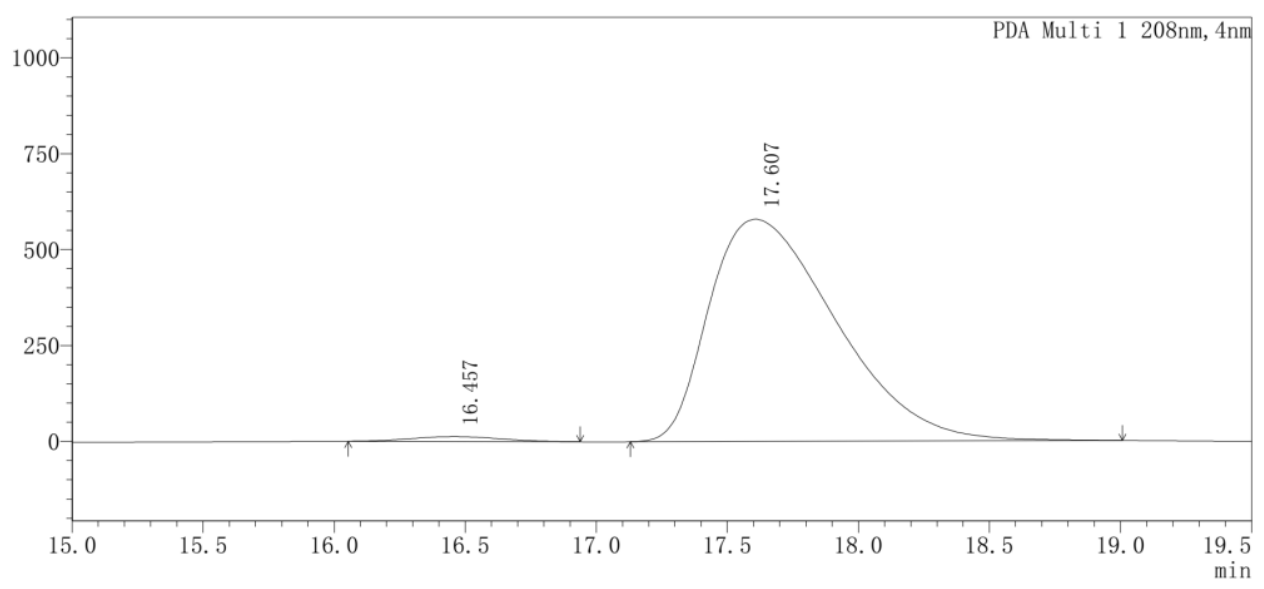

〈Peak Results >

PDA Ch1 208nm

\begin{tabular}{|c|c|c|c|c|}
\hline Index & Time/min & Height/mAU & Quantity/Area & Area \%/\% \\
\hline 1 & 16.457 & 12722 & 305031 & 1.541 \\
\hline 2 & 17.607 & 579314 & 19488649 & 98.459 \\
\hline
\end{tabular}




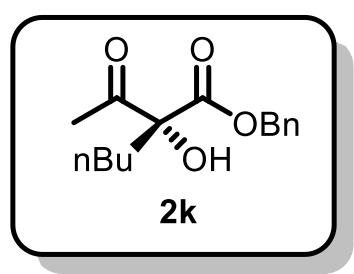

HPLC (ChiralPak AS-H, 2\% i-PrOH in hexanes, $0.8 \mathrm{~mL} / \mathrm{min}, 208 \mathrm{~nm}$ )

$\langle$ Chromatogram〉

$\mathrm{mAU}$

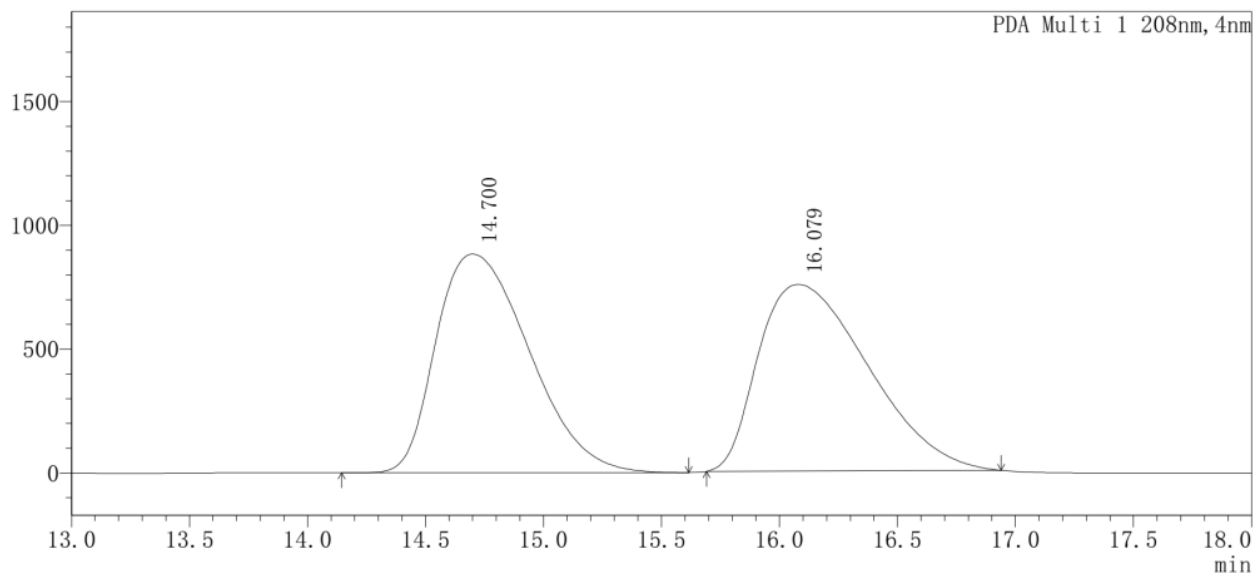

〈Peak Results〉

PDA Ch1 208nm

\begin{tabular}{|c|c|c|c|c|}
\hline Index & Time/min & Height/mAU & Quantity/Area & Area \%/\% \\
\hline 1 & 14.700 & 882918 & 24234941 & 49.850 \\
\hline 2 & 16.079 & 753652 & 24380885 & 50.150 \\
\hline
\end{tabular}

\section{〈Chromatogram〉}

mAU

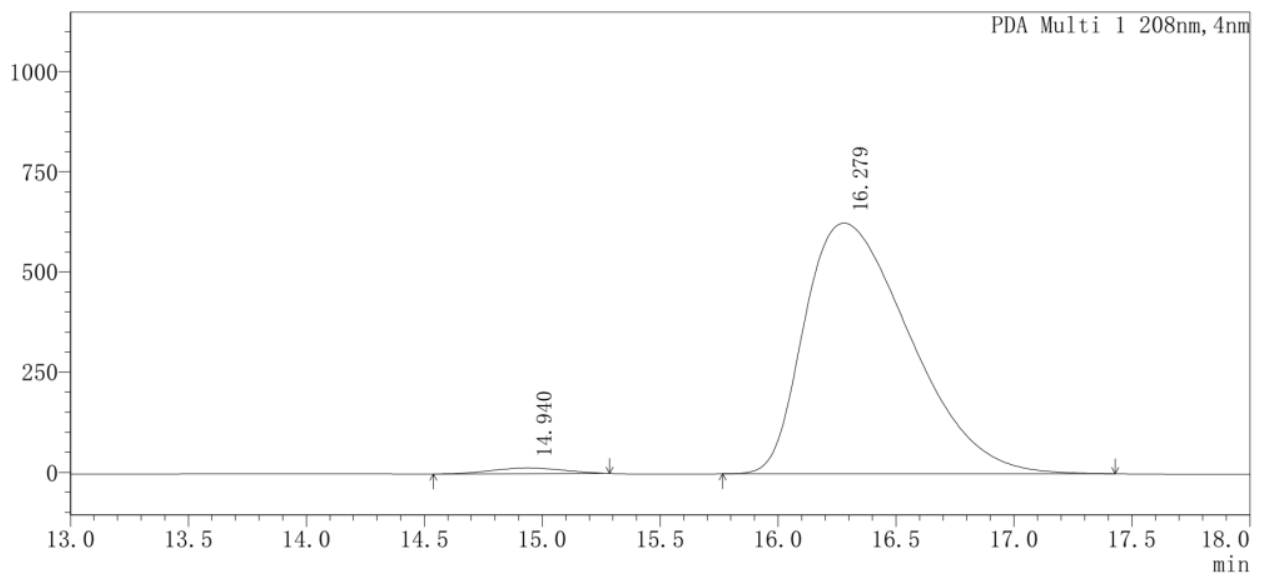

〈Peak Results

PDA Ch1 208nm

\begin{tabular}{|c|c|c|c|c|}
\hline Index & Time/min & Height/mAU & Quantity/Area & Area \%/\% \\
\hline 1 & 14.940 & 14138 & 302725 & 1.526 \\
\hline 2 & 16.279 & 626015 & 19531851 & 98.474 \\
\hline
\end{tabular}




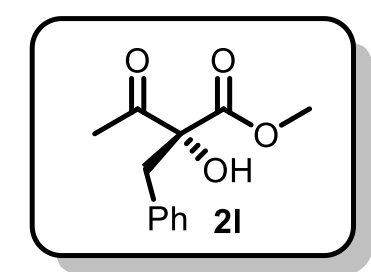

HPLC (ChiralPak AS-H, 5\% i-PrOH in hexanes, $0.8 \mathrm{~mL} / \mathrm{min}, 209 \mathrm{~nm}$ )

$\langle$ Chromatogram〉

$\mathrm{mAU}$

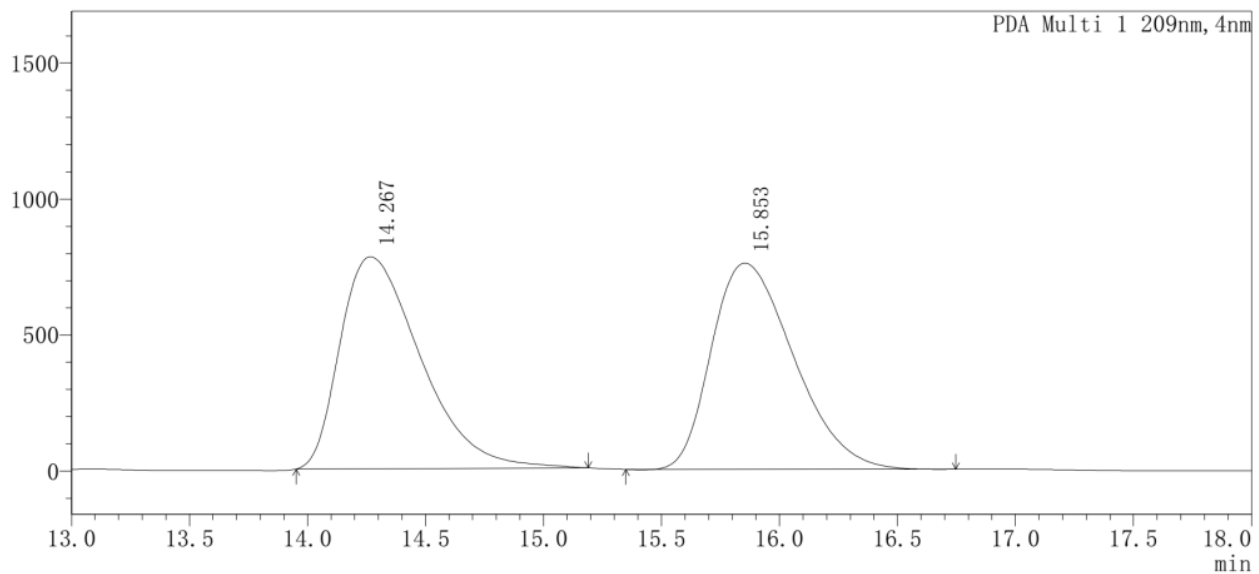

〈Peak Results〉

PDA Ch1 209nm

\begin{tabular}{|c|c|c|c|c|}
\hline Index & Time/min & Height/mAU & Quantity/Area & Area \%/\% \\
\hline 1 & 14.267 & 779317 & 18479986 & 50.121 \\
\hline 2 & 15.853 & 758418 & 18391034 & 49.879 \\
\hline
\end{tabular}

$\langle$ Chromatogram〉

$\mathrm{mAU}$

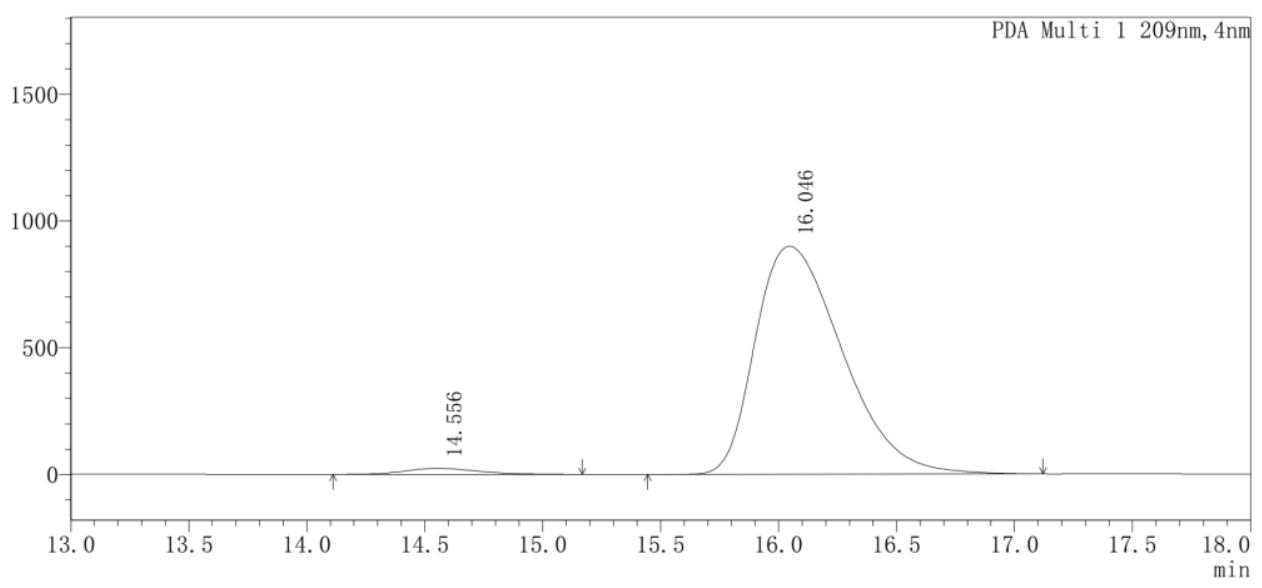

〈Peak Results〉

PDA Ch1 209nm

\begin{tabular}{|c|c|c|c|c|}
\hline Index & Time/min & Height/mAU & Quantity/Area & Area \%/\% \\
\hline 1 & 14.556 & 23425 & 506309 & 2.146 \\
\hline 2 & 16.046 & 899934 & 23085948 & 97.854 \\
\hline
\end{tabular}




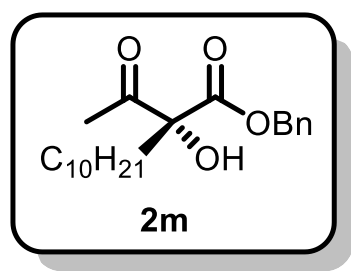

HPLC (ChiralPak AS-H*2, 5\% i-PrOH in hexanes, $0.8 \mathrm{~mL} / \mathrm{min}, 209 \mathrm{~nm}$ )

$\langle$ Chromatogram〉

$\mathrm{mAU}$

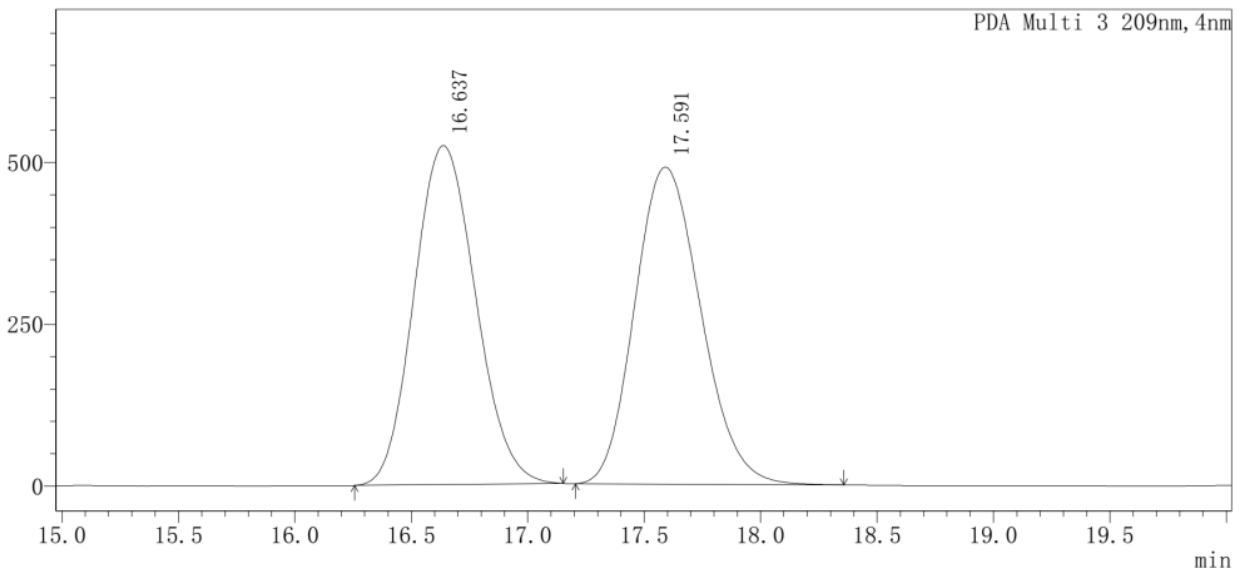

$\langle$ Peak Results〉

PDA Ch1 205nm

\begin{tabular}{|c|c|c|c|c|}
\hline Index & Time/min & Height/mAU & Quantity/Area & Area \%/\% \\
\hline 1 & 16.637 & 474923 & 8924421 & 50.244 \\
\hline 2 & 17.591 & 442913 & 8837774 & 49.756 \\
\hline
\end{tabular}

〈Chromatogram〉

mAU

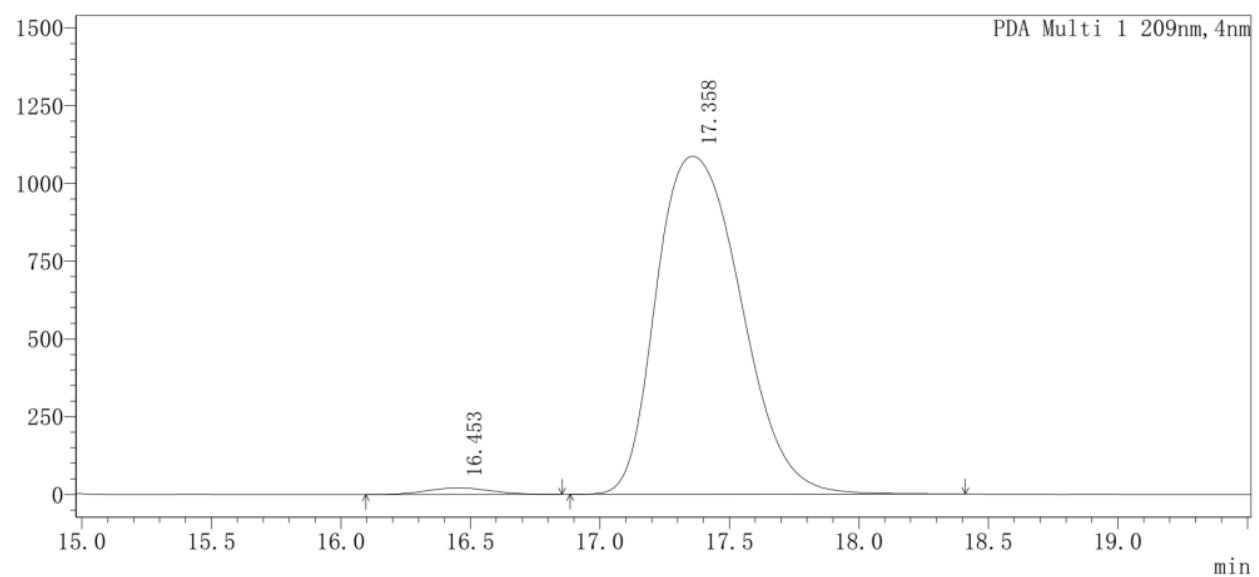

$\langle$ Peak Results $>$

PDA Ch1 209nm

\begin{tabular}{|c|c|c|c|c|}
\hline Index & Time/min & Height/mAU & Quantity/Area & Area $\% / \%$ \\
\hline 1 & 16.453 & 21407 & 364190 & 1.451 \\
\hline 2 & 17.358 & 1086207 & 24734513 & 98.549 \\
\hline
\end{tabular}




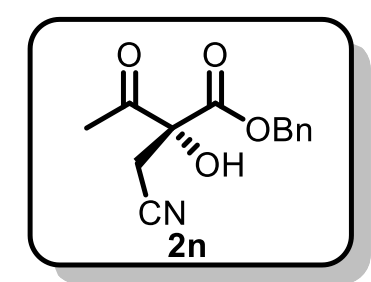

HPLC (ChiralPak AS-H, 10\% i-PrOH in hexanes, $1.0 \mathrm{~mL} / \mathrm{min}, 209 \mathrm{~nm}$ )

〈Chromatogram〉

mAU

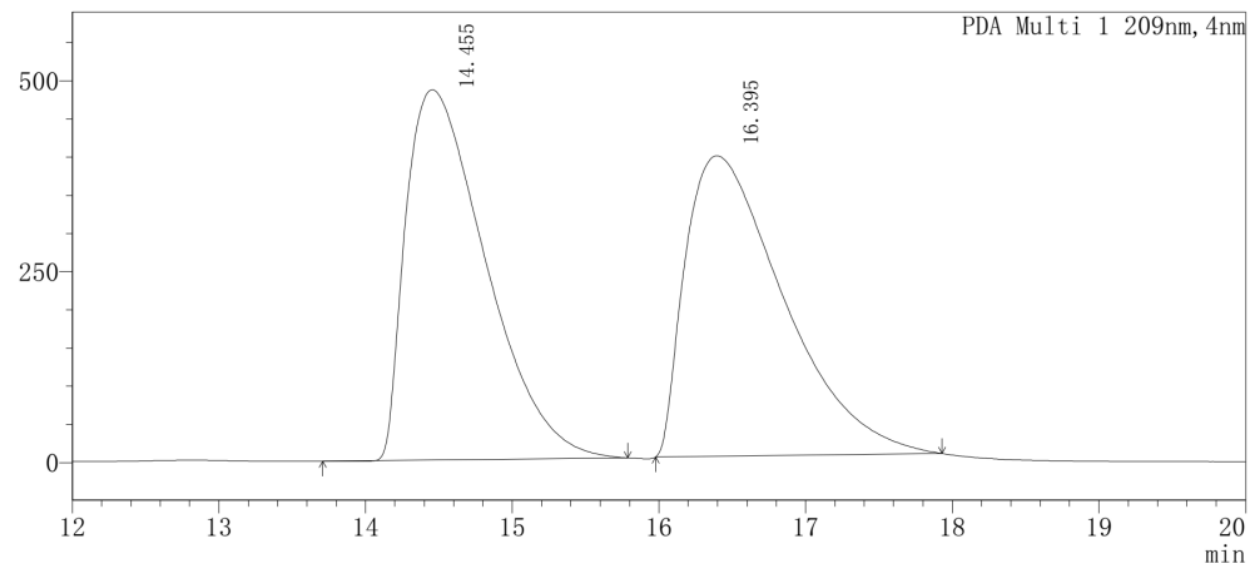

〈Peak Results〉

PDA Ch1 209nm

\begin{tabular}{|c|c|c|c|c|}
\hline Index & Time/min & Height/mAU & Quantity/Area & Area \%/\% \\
\hline 1 & 14.455 & 484926 & 18405400 & 50.051 \\
\hline 2 & 16.395 & 393332 & 18367620 & 49.949 \\
\hline
\end{tabular}

〈Chromatogram〉

$\mathrm{mAU}$

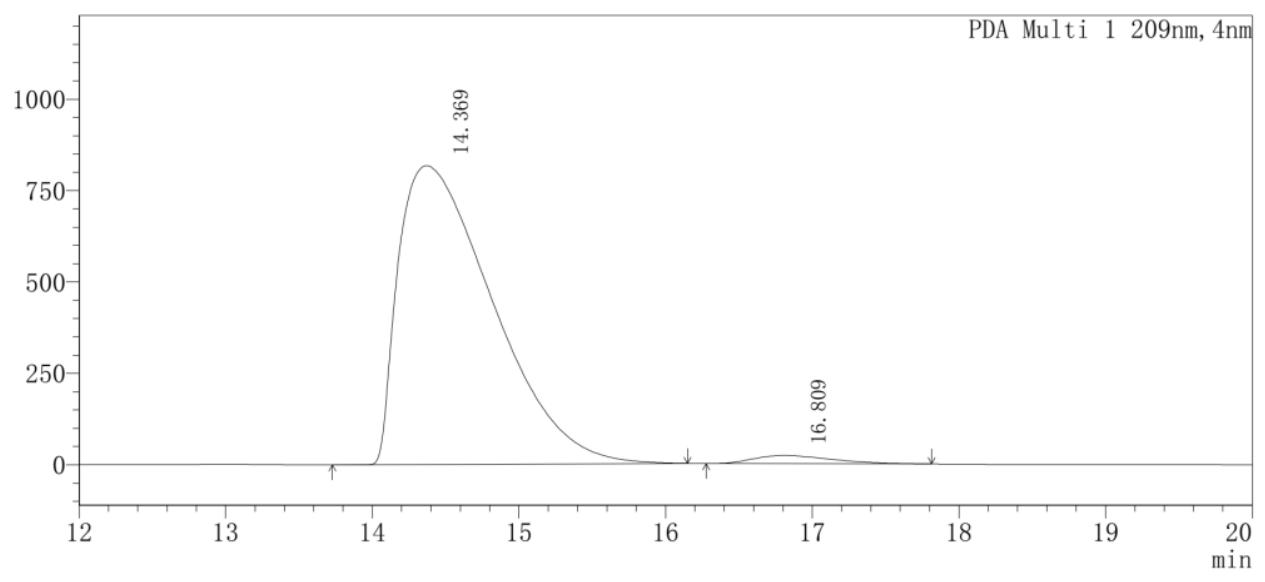

$\langle$ Peak Results〉

PDA Ch1 209nm

\begin{tabular}{|c|c|c|c|c|}
\hline Index & Time/min & Height/mAU & Quantity/Area & Area \%/\% \\
\hline 1 & 14.369 & 816989 & 36585123 & 97.769 \\
\hline 2 & 16.809 & 22336 & 834733 & 2.231 \\
\hline
\end{tabular}




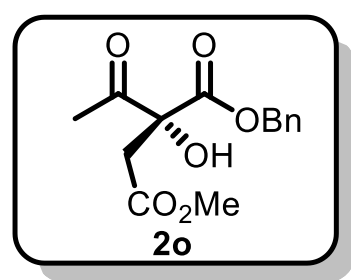

HPLC (ChiralPak OJ-H, 10\% i-PrOH in hexanes, $1.0 \mathrm{~mL} / \mathrm{min}, 209 \mathrm{~nm}$ )

〈Chromatogram〉

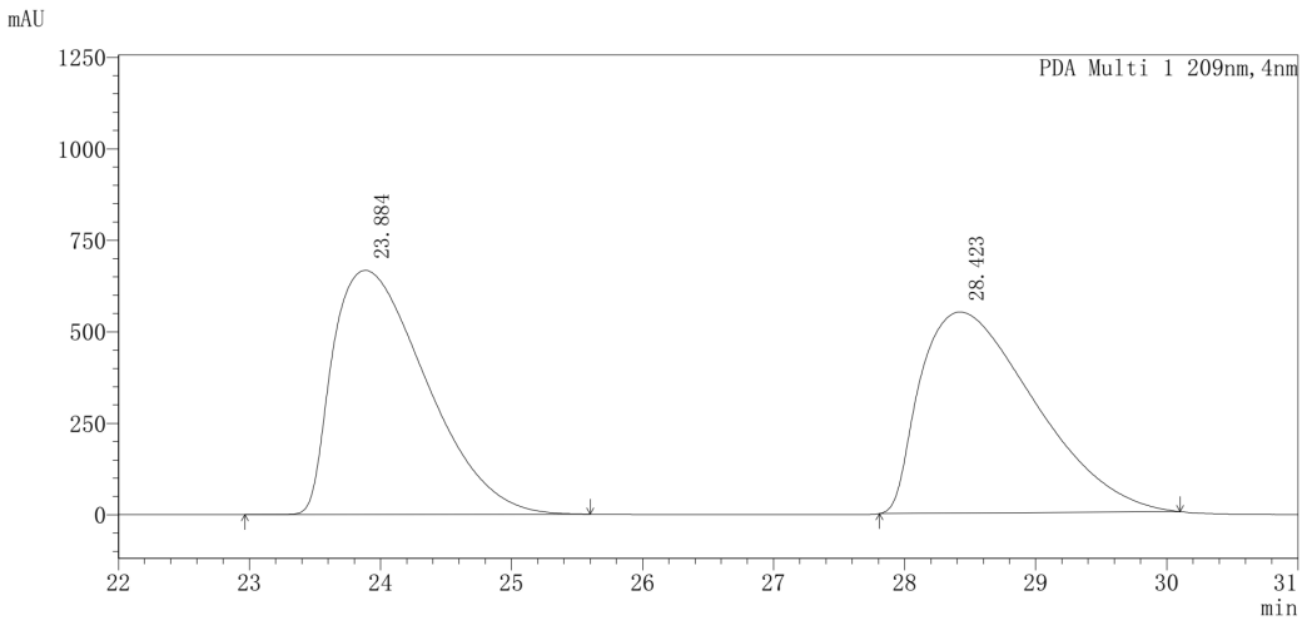

〈Peak Results

PDA Ch1 209nm

\begin{tabular}{|c|c|c|c|c|}
\hline Index & Time/min & Height/mAU & Quantity/Area & Area \%/\% \\
\hline 1 & 23.884 & 666959 & 33123182 & 49.826 \\
\hline 2 & 28.423 & 549229 & 33354604 & 50.174 \\
\hline
\end{tabular}

$\langle$ Chromatogram〉

$\mathrm{mAU}$

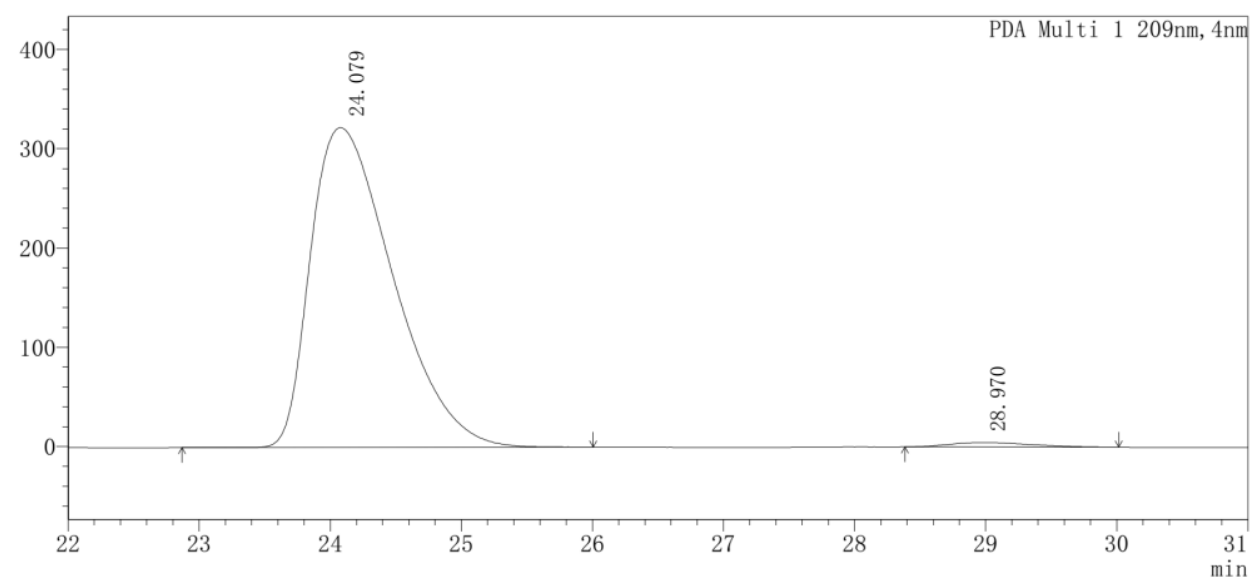

〈Peak Results>

PDA Ch1 209nm

\begin{tabular}{|c|c|c|c|c|}
\hline Index & Time/min & Height/mAU & Quantity/Area & Area \%/\% \\
\hline 1 & 24.079 & 322159 & 14078982 & 98.594 \\
\hline 2 & 28.970 & 4595 & 200773 & 1.406 \\
\hline
\end{tabular}




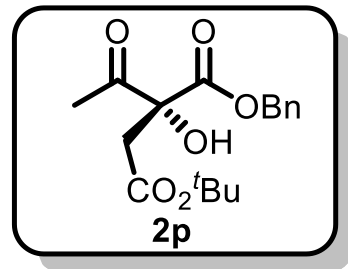

HPLC (ChiralPak OJ-H, 5\% i-PrOH in hexanes, $1.0 \mathrm{~mL} / \mathrm{min}, 208 \mathrm{~nm}$ )

$\langle$ Chromatogram〉

$\mathrm{mAU}$

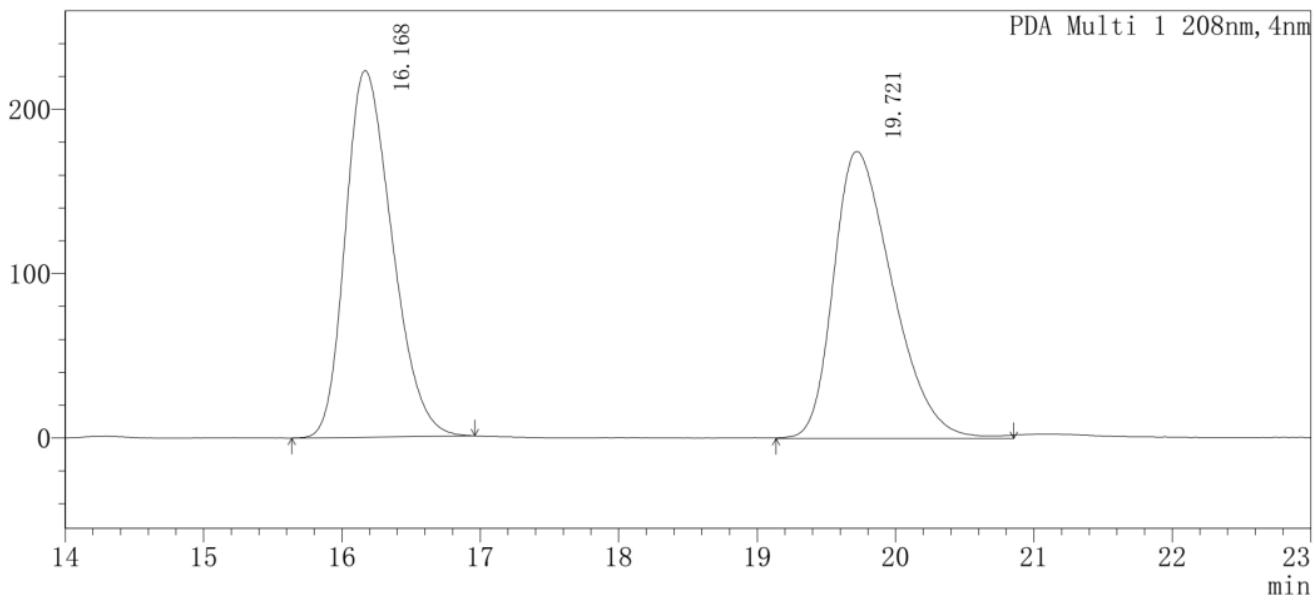

〈Peak Results〉

PDA Ch1 208nm

\begin{tabular}{|c|c|c|c|c|}
\hline Index & Time/min & Height/mAU & Quantity/Area & Area \%/\% \\
\hline 1 & 16.168 & 223205 & 5243909 & 50.220 \\
\hline 2 & 19.721 & 174847 & 5197904 & 49.780 \\
\hline
\end{tabular}

$\langle$ Chromatogram〉

mAU

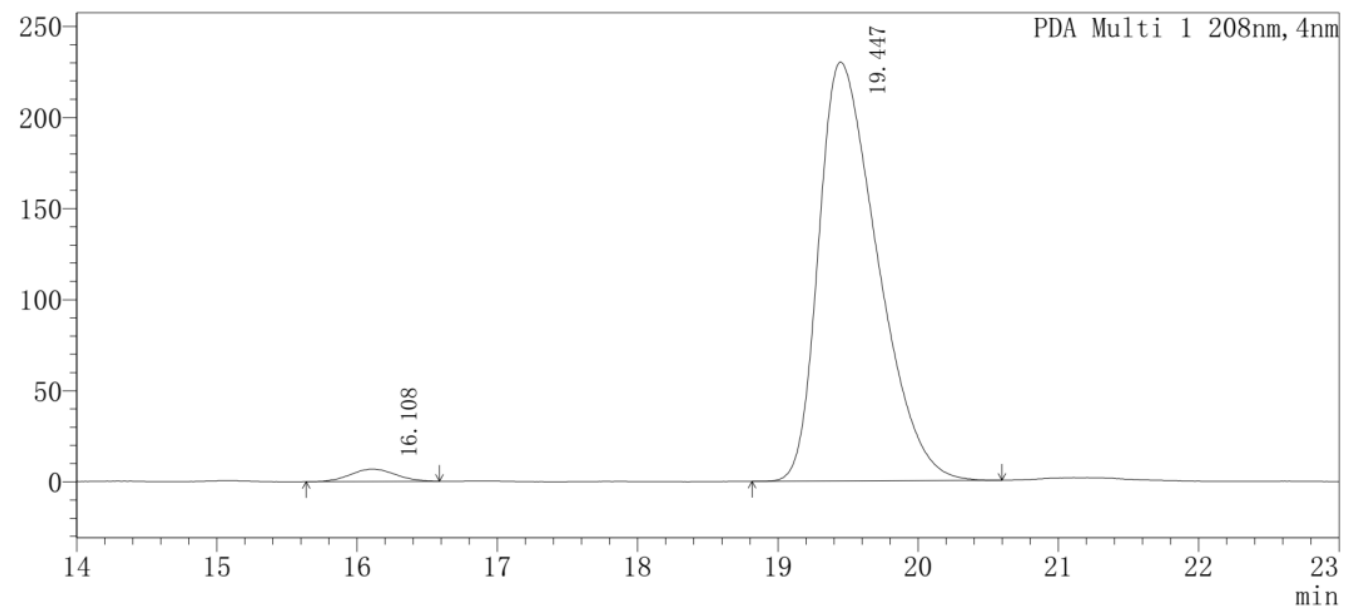

$\langle$ Peak Results〉

PDA Ch1 208nm

\begin{tabular}{|c|c|c|c|c|}
\hline Index & Time/min & Height/mAU & Quantity/Area & Area \%/\% \\
\hline 1 & 16.108 & 6833 & 151374 & 2.210 \\
\hline 2 & 19.447 & 229965 & 6698690 & 97.790 \\
\hline
\end{tabular}




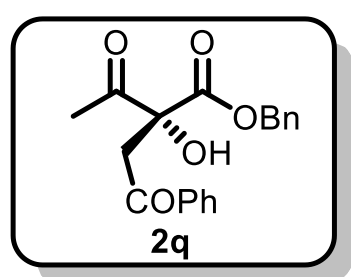

HPLC (ChiralPak OJ-H, 5\% i-PrOH in hexanes, $1.0 \mathrm{~mL} / \mathrm{min}, 242 \mathrm{~nm}$ )

$\langle$ Chromatogram

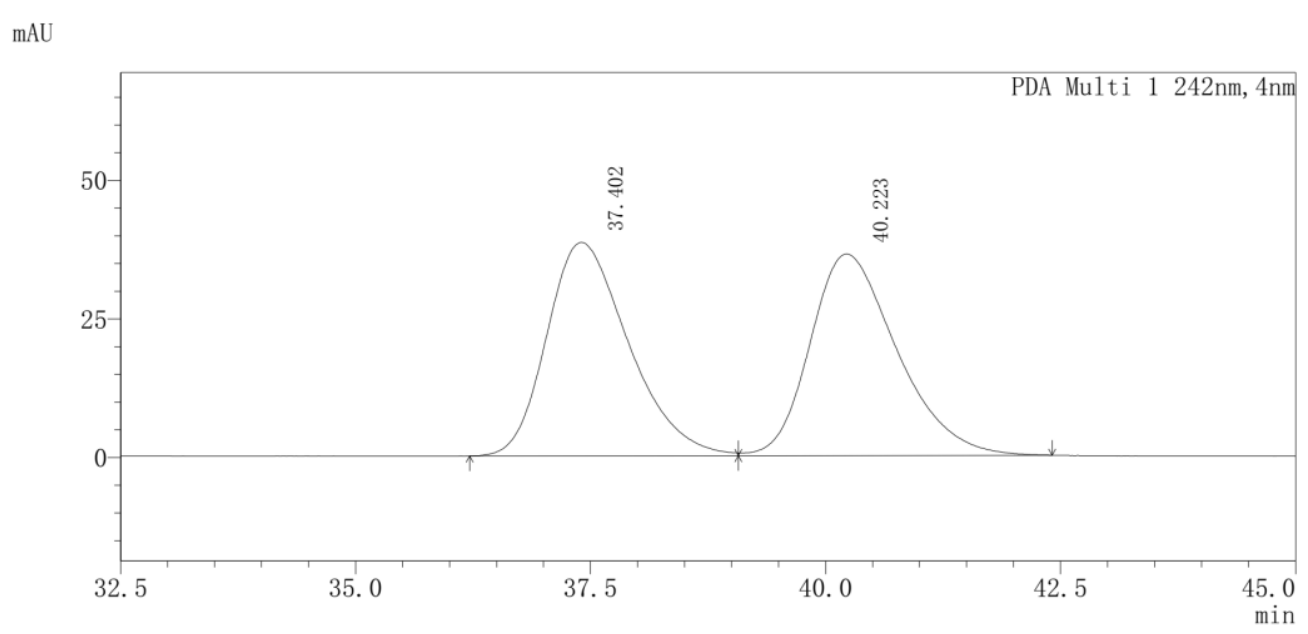

〈Peak Results〉

PDA Ch1 242nm

\begin{tabular}{|c|c|c|c|c|}
\hline Index & Time/min & Height/mAU & Quantity/Area & Area \%/\% \\
\hline 1 & 37.402 & 38520 & 2332560 & 50.007 \\
\hline 2 & 40.223 & 36356 & 2331877 & 49.993 \\
\hline
\end{tabular}

$\langle$ Chromatogram〉

$\mathrm{mAU}$

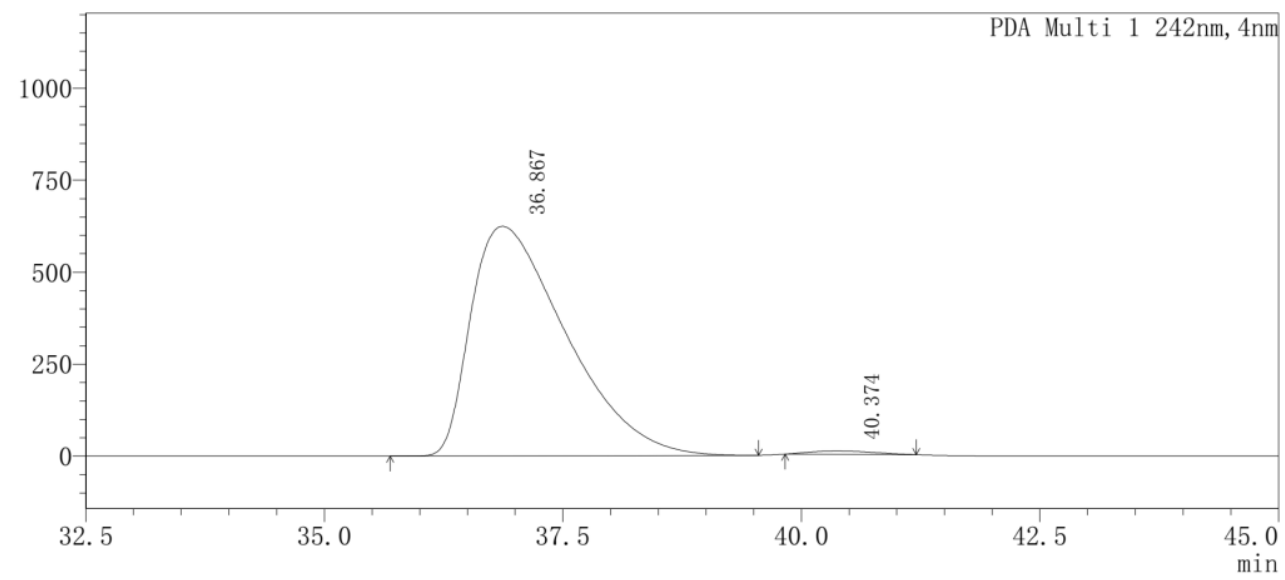

〈Peak Results>

PDA Ch1 242nm

\begin{tabular}{|c|c|c|c|c|}
\hline Index & Time/min & Height/mAU & Quantity/Area & Area \%/\% \\
\hline 1 & 36.867 & 623917 & 43277961 & 99.004 \\
\hline 2 & 40.374 & 9353 & 435373 & 0.996 \\
\hline
\end{tabular}




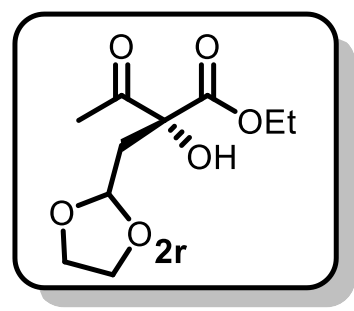

HPLC (ChiralPak AD-H, 5\% i-PrOH in hexanes, $0.8 \mathrm{~mL} / \mathrm{min}, 205 \mathrm{~nm}$ )

\section{$\langle$ Chromatogram〉}

mAU

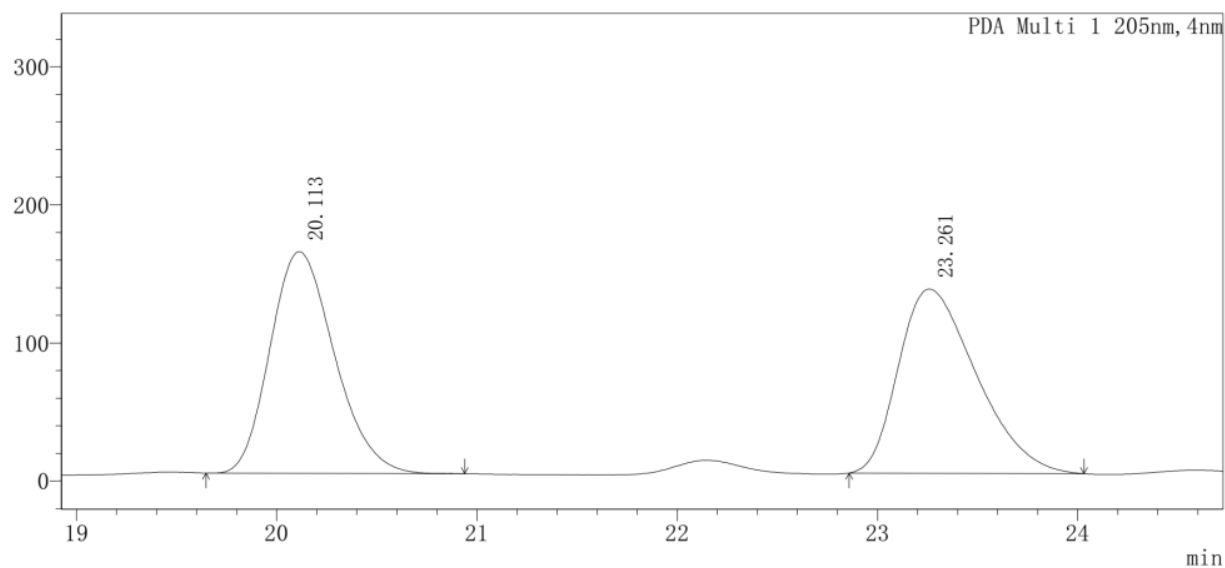

〈Peak Results〉

PDA Ch1 205nm

\begin{tabular}{|c|c|c|c|c|}
\hline Index & Time/min & Height/mAU & Quantity/Area & Area \%/\% \\
\hline 1 & 20.113 & 160483 & 3577703 & 49.874 \\
\hline 2 & 23.261 & 133407 & 3595841 & 50.126 \\
\hline
\end{tabular}

$\langle$ Chromatogram〉

$\mathrm{mAU}$

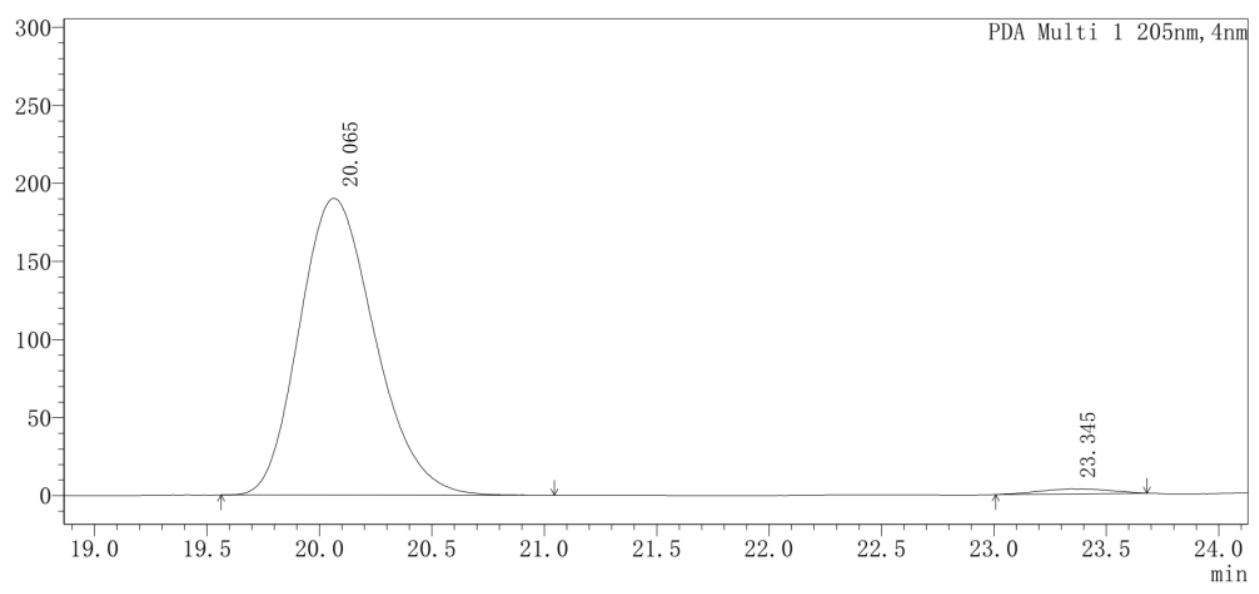

〈Peak Results〉

PDA Ch1 205nm

\begin{tabular}{|c|c|c|c|c|}
\hline PDdex & Time/min & Height/mAU & Quantity/Area & Area \%/\% \\
\hline 1 & 20.065 & 190192 & 4436221 & 98.443 \\
\hline 2 & 23.345 & 3245 & 70165 & 1.557 \\
\hline
\end{tabular}




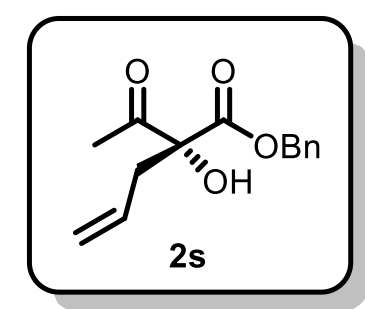

HPLC (ChiralPak As-H*2, 5\% i-PrOH in hexanes, $0.8 \mathrm{~mL} / \mathrm{min}, 208 \mathrm{~nm}$ )

$\langle$ Chromatogram〉

$\mathrm{mAU}$

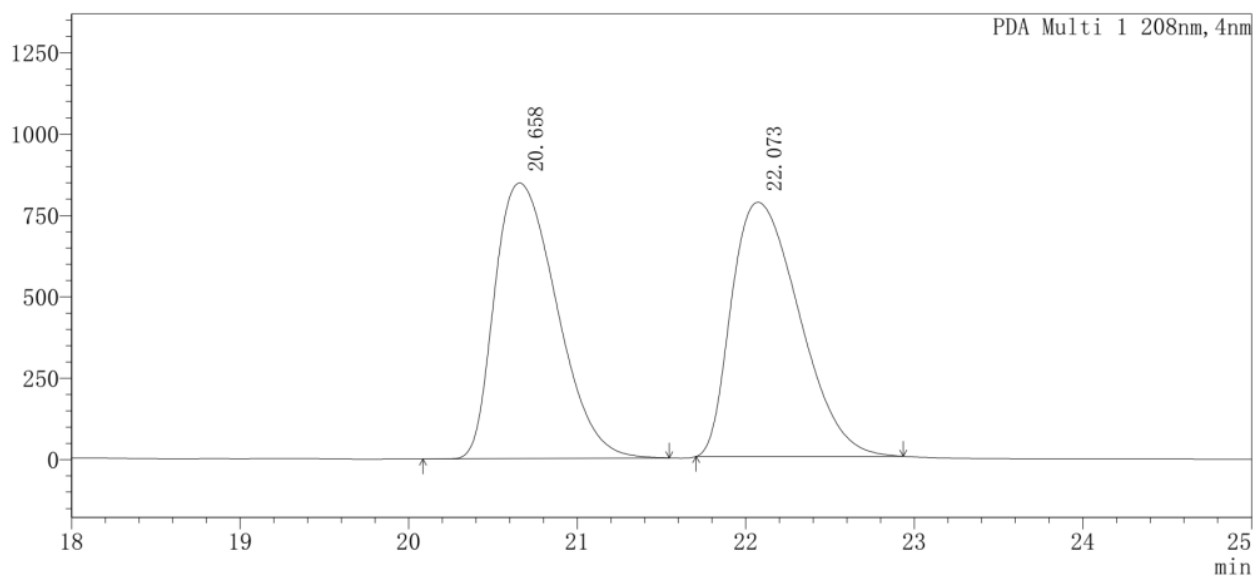

〈Peak Results〉

PDA Ch1 208nm

\begin{tabular}{|c|c|c|c|c|}
\hline Index & Time/min & Height/mAU & Quantity/Area & Area \%/\% \\
\hline 1 & 20.658 & 846974 & 21690847 & 49.814 \\
\hline 2 & 22.073 & 780896 & 21852533 & 50.186 \\
\hline
\end{tabular}

〈Chromatogram〉

$\mathrm{mAU}$

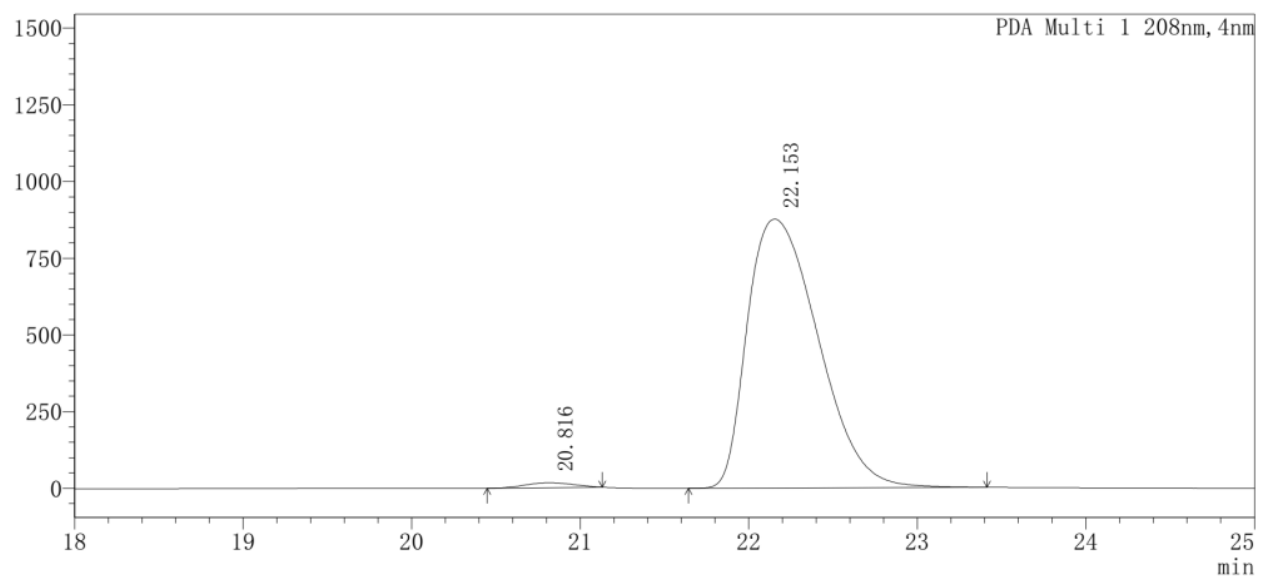

〈Peak Results>

PDA Ch1 208nm

\begin{tabular}{|c|c|c|c|c|}
\hline Index & Time/min & Height/mAU & Quantity/Area & Area \%/\% \\
\hline 1 & 20.816 & 15955 & 316229 & 1.220 \\
\hline 2 & 22.153 & 876486 & 25599641 & 98.780 \\
\hline
\end{tabular}




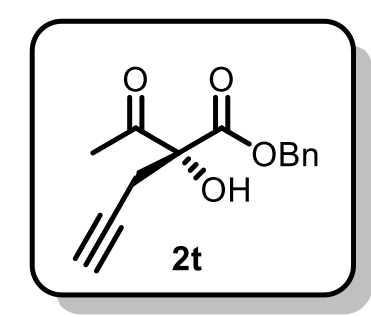

HPLC (ChiralPak As-H, 2\% i-PrOH in hexanes, $0.8 \mathrm{~mL} / \mathrm{min}, 208 \mathrm{~nm}$ )

$\langle$ Chromatogram〉

$\mathrm{mAU}$

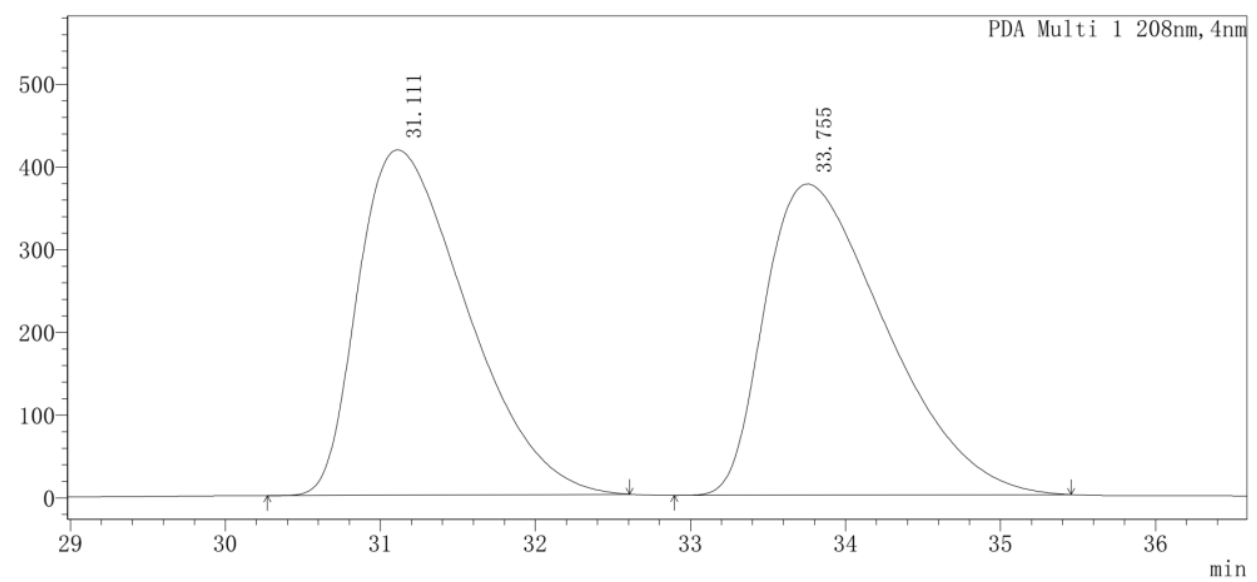

$\langle$ Peak Results $\rangle$

PDA Ch1 208nm

\begin{tabular}{|c|c|c|c|c|}
\hline Index & Time/min & Height/mAU & Quantity/Area & Area \%/\% \\
\hline 1 & 31.111 & 417250 & 20331709 & 49.824 \\
\hline 2 & 33.755 & 376010 & 20475126 & 50.176 \\
\hline
\end{tabular}

$\langle$ Chromatogram〉

$\mathrm{mAU}$

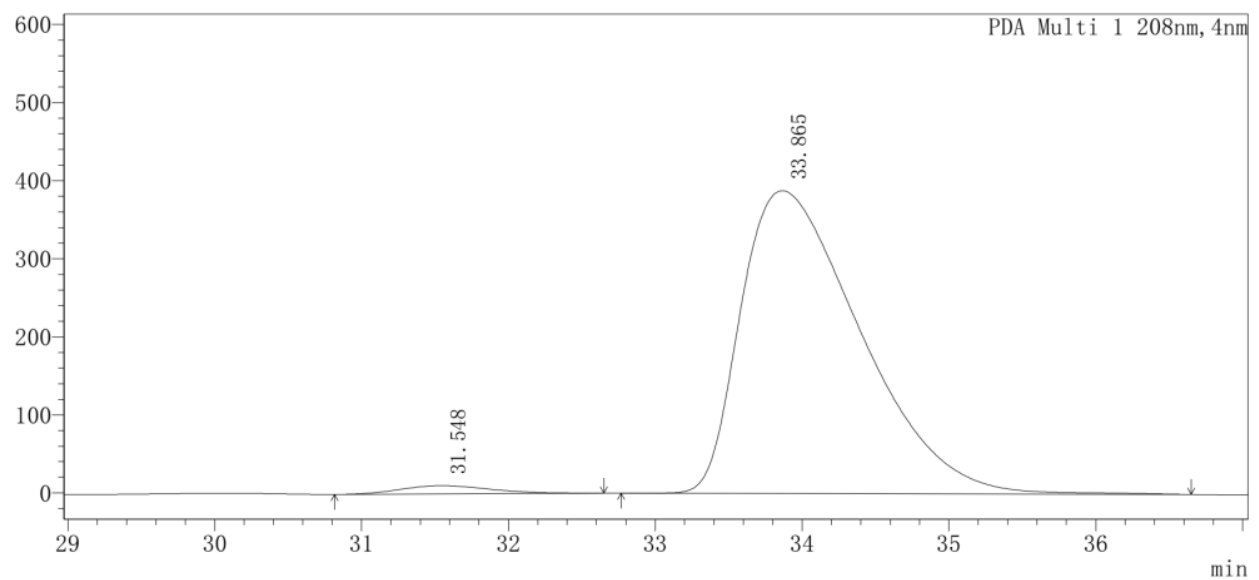

〈Peak Results〉

PDA Ch1 208nm

\begin{tabular}{|c|c|c|c|c|}
\hline Index & Time/min & Height/mAU & Quantity/Area & Area \%/\% \\
\hline 1 & 31.548 & 10501 & 443688 & 1.980 \\
\hline 2 & 33.865 & 387616 & 21962002 & 98.020 \\
\hline
\end{tabular}




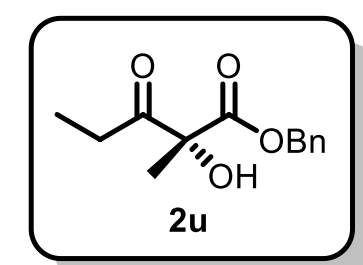

HPLC (ChiralPak OJ-H, 5\% i-PrOH in hexanes, $1.0 \mathrm{~mL} / \mathrm{min}, 208 \mathrm{~nm}$ )

〈Chromatogram〉

$\mathrm{mAU}$

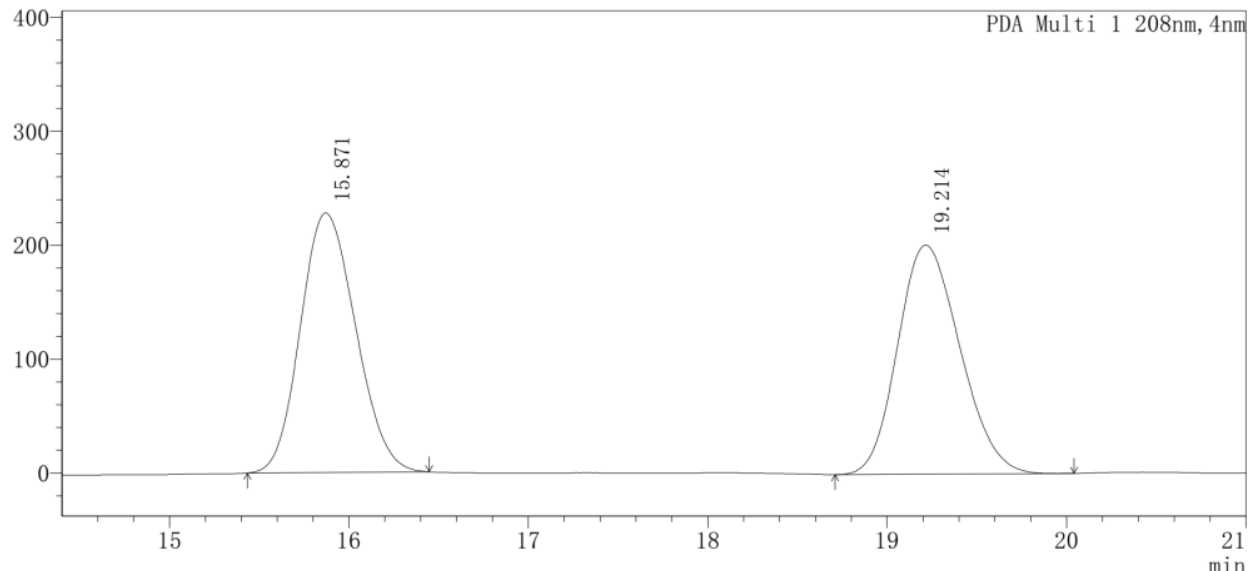

〈Peak Results〉

PDA Ch1 208nm

\begin{tabular}{|c|c|c|c|c|}
\hline Index & Time/min & Height/mAU & Quantity/Area & Area \%/\% \\
\hline 1 & 15.871 & 228055 & 4885484 & 50.151 \\
\hline 2 & 19.214 & 201271 & 4856132 & 49.849 \\
\hline
\end{tabular}

〈Chromatogram〉

mAU

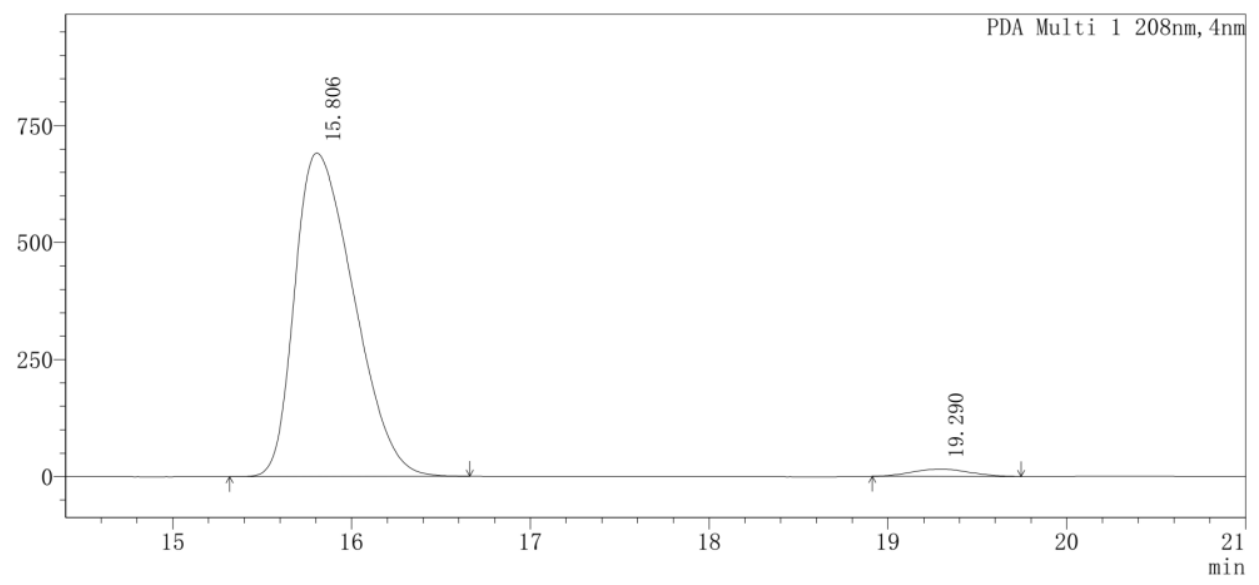

〈Peak Results>

PDA Ch1 208nm

\begin{tabular}{|c|c|c|c|c|}
\hline Index & Time/min & Height/mAU & Quantity/Area & Area $\% / \%$ \\
\hline 1 & 15.806 & 691489 & 15884564 & 97.826 \\
\hline 2 & 19.290 & 15816 & 353027 & 2.174 \\
\hline
\end{tabular}




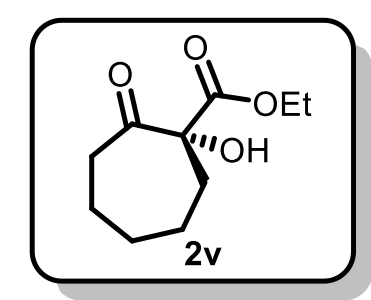

HPLC (ChiralPak OD-H, 5\% i-PrOH in hexanes, $0.8 \mathrm{~mL} / \mathrm{min}, 205 \mathrm{~nm}$ )

〈Chromatogram〉

$\mathrm{mAU}$

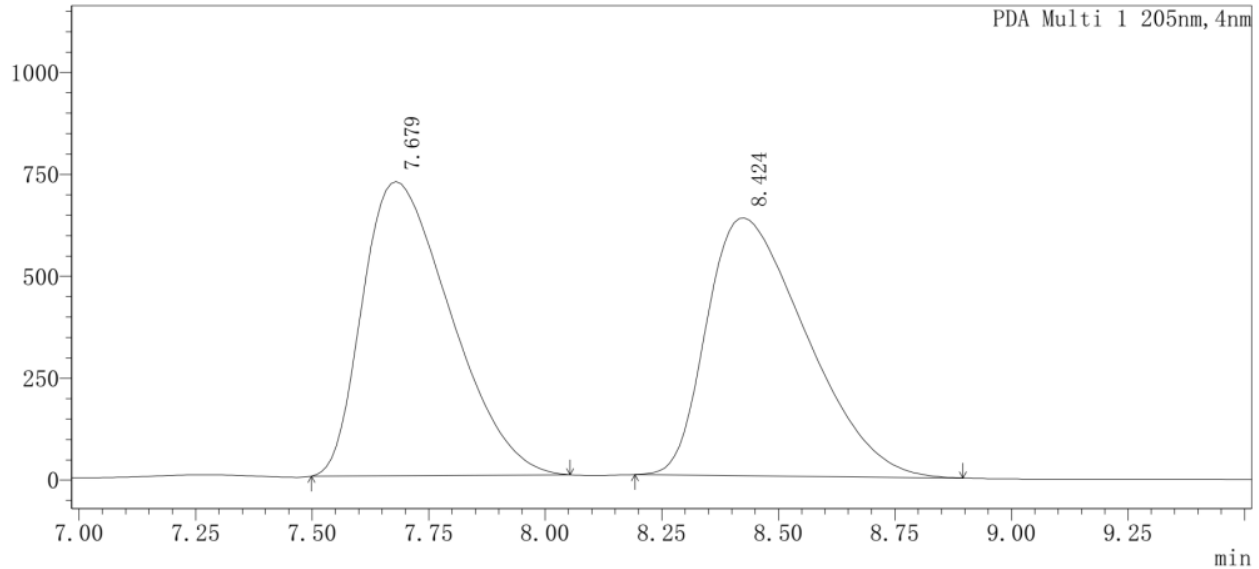

〈Peak Results〉

PDA Ch1 205nm

\begin{tabular}{|c|c|c|c|c|}
\hline Index & Time/min & Height/mAU & Quantity/Area & Area \%/\% \\
\hline 1 & 7.679 & 721578 & 9484724 & 50.221 \\
\hline 2 & 8.424 & 632670 & 9401410 & 49.779 \\
\hline
\end{tabular}

$\langle$ Chromatogram〉

$\mathrm{mAU}$

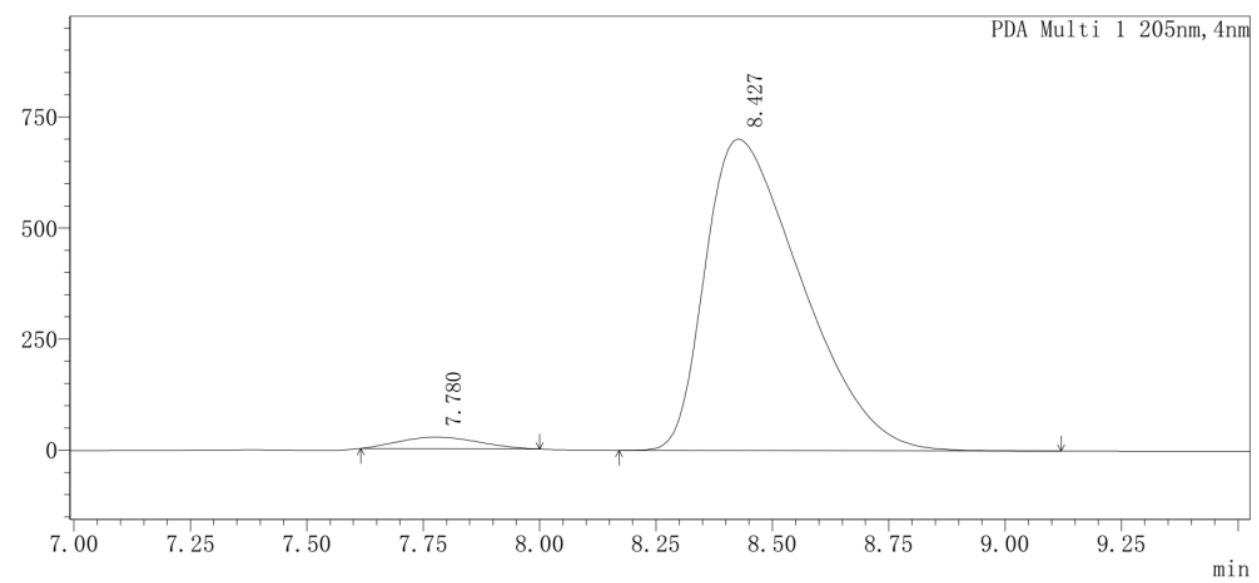

〈Peak Results〉

PDA Ch1 205nm

\begin{tabular}{|c|c|c|c|c|}
\hline Index & Time/min & Height/mAU & Quantity/Area & Area \%/\% \\
\hline 1 & 7.780 & 26379 & 315212 & 3.018 \\
\hline 2 & 8.427 & 701920 & 10128390 & 96.982 \\
\hline
\end{tabular}




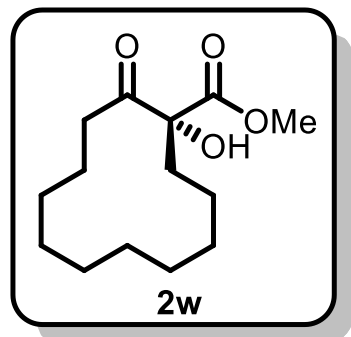

HPLC (ChiralPak As-H*2, 5\% i-PrOH in hexanes, $0.8 \mathrm{~mL} / \mathrm{min}, 205 \mathrm{~nm}$ )

$\langle$ Chromatogram〉

$\mathrm{mAU}$

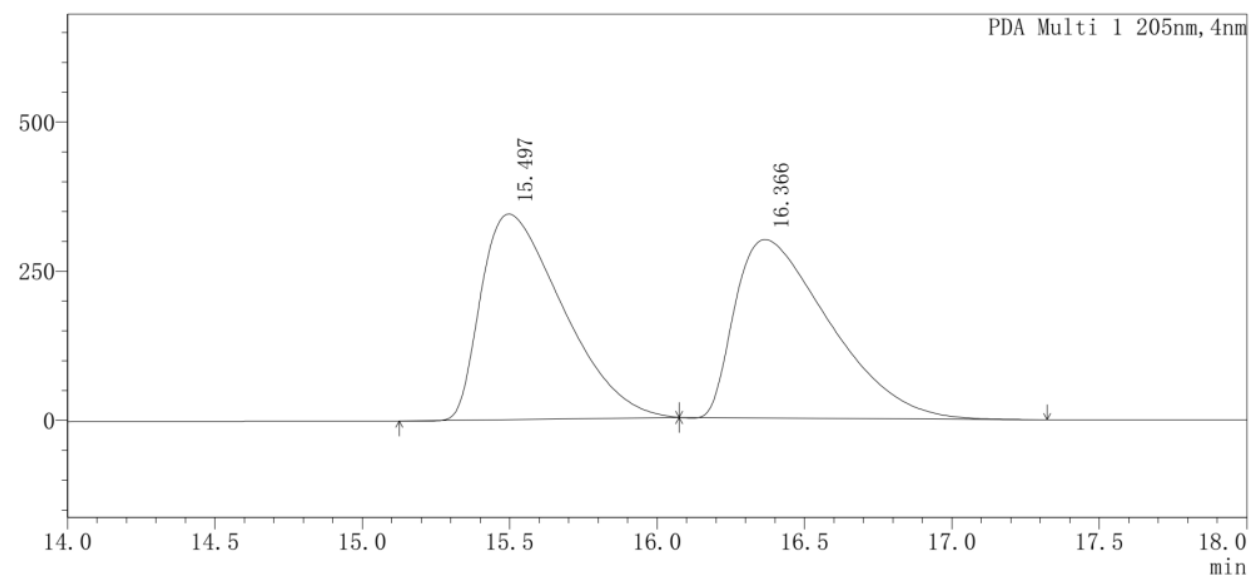

〈Peak Results〉

PDA Ch1 205nm

\begin{tabular}{|c|c|c|c|c|}
\hline Index & Time/min & Height/mAU & Quantity/Area & Area \%/\% \\
\hline 1 & 15.497 & 344562 & 6642583 & 49.852 \\
\hline 2 & 16.366 & 298851 & 6682070 & 50.148 \\
\hline
\end{tabular}

$\langle$ Chromatogram〉

mAU

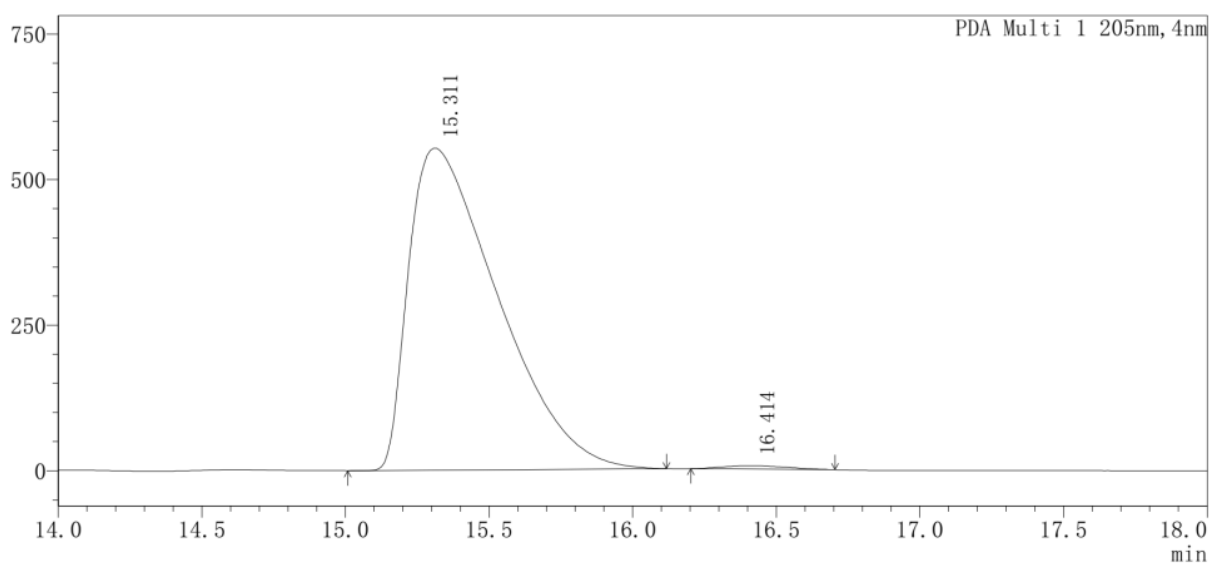

〈Peak Results>

PDA Ch1 205nm

\begin{tabular}{|c|c|c|c|c|}
\hline Index & Time/min & Height/mAU & Quantity/Area & Area \%/\% \\
\hline 1 & 15.311 & 553019 & 11962931 & 99.229 \\
\hline 2 & 16.414 & 5904 & 92976 & 0.771 \\
\hline
\end{tabular}




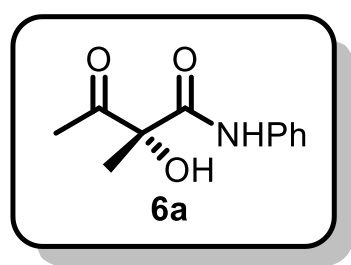

HPLC (ChiralPak As-H, 5\% i-PrOH in hexanes, $0.8 \mathrm{~mL} / \mathrm{min}, 206 \mathrm{~nm}$ )

〈Chromatogram〉

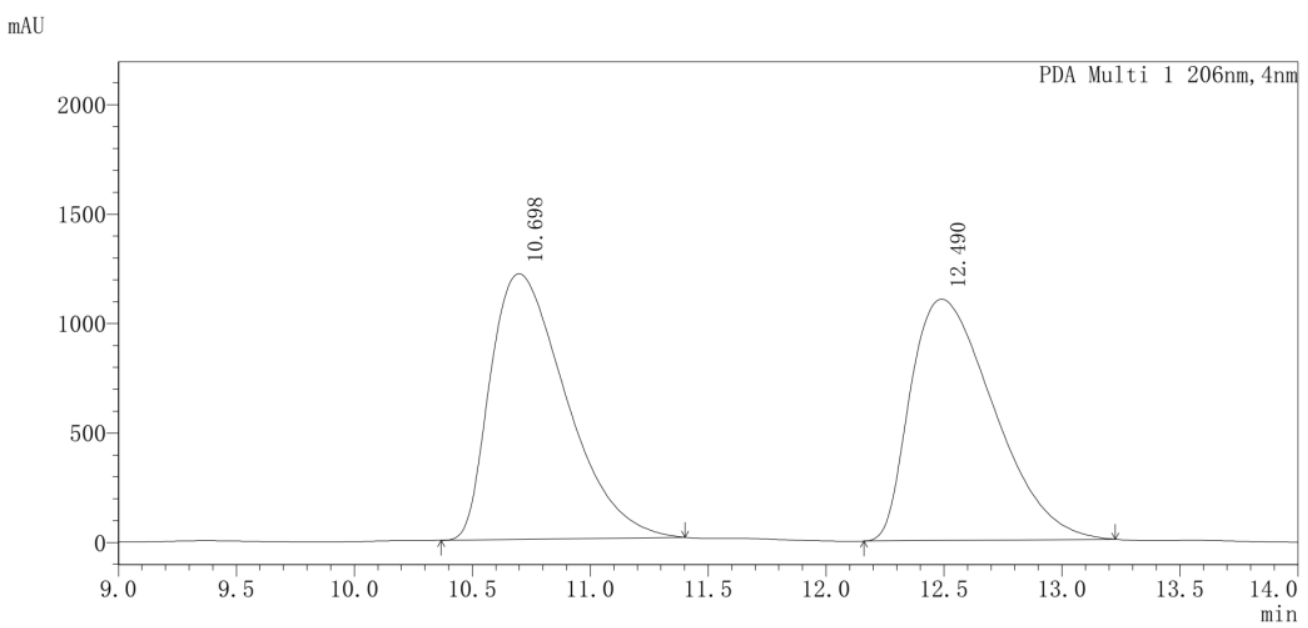

〈Peak Results〉

PDA Ch1 206nm

\begin{tabular}{|c|c|c|c|c|}
\hline Index & Time/min & Height/mAU & Quantity/Area & Area \%/\% \\
\hline 1 & 10.698 & 1214854 & 27185355 & 50.037 \\
\hline 2 & 12.490 & 1103585 & 27144935 & 49.963 \\
\hline
\end{tabular}

$\langle$ Chromatogram〉

$\mathrm{mAU}$

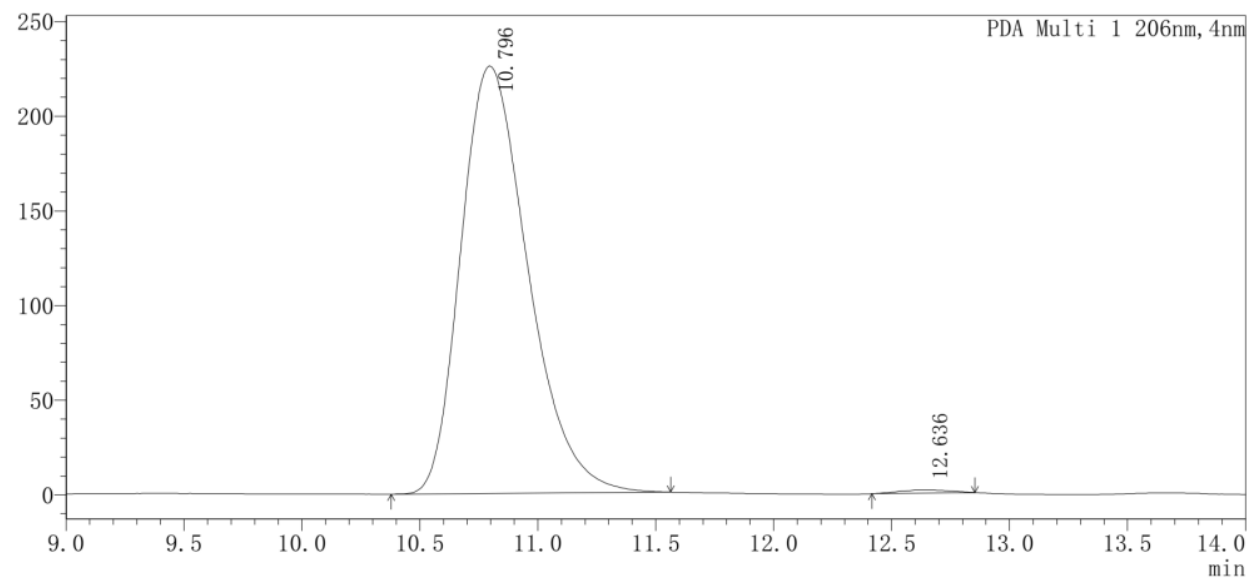

$\langle$ Peak Results $\rangle$

PDA Ch1 206nm

\begin{tabular}{|c|c|c|c|c|}
\hline Index & Time/min & Height/mAU & Quantity/Area & Area \%/\% \\
\hline 1 & 10.796 & 225901 & 4473485 & 99.438 \\
\hline 2 & 12.636 & 1711 & 25288 & 0.562 \\
\hline
\end{tabular}




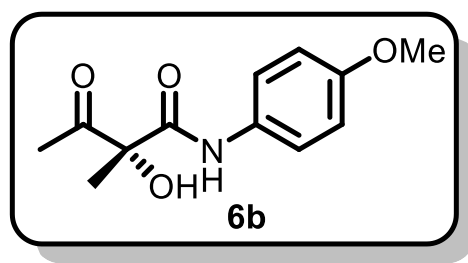

HPLC (ChiralPak OJ-H, 10\% i-PrOH in hexanes, $1.0 \mathrm{~mL} / \mathrm{min}, 206 \mathrm{~nm}$ )

$\langle$ Chromatogram〉

$\mathrm{mAU}$

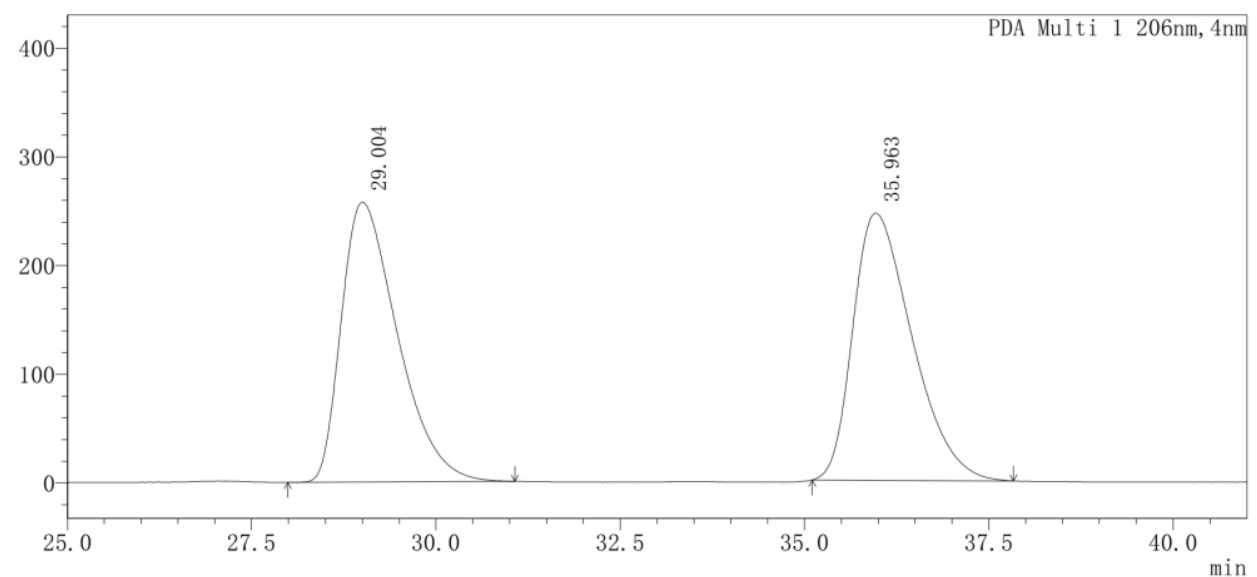

$\langle$ Peak Results $\rangle$

PDA Ch1 206nm

\begin{tabular}{|c|c|c|c|c|}
\hline Index & Time/min & Height/mAU & Quantity/Area & Area \%/\% \\
\hline 1 & 29.004 & 257646 & 13665341 & 49.856 \\
\hline 2 & 35.963 & 245837 & 13744134 & 50.144 \\
\hline
\end{tabular}

$\langle$ Chromatogram〉

$\mathrm{mAU}$

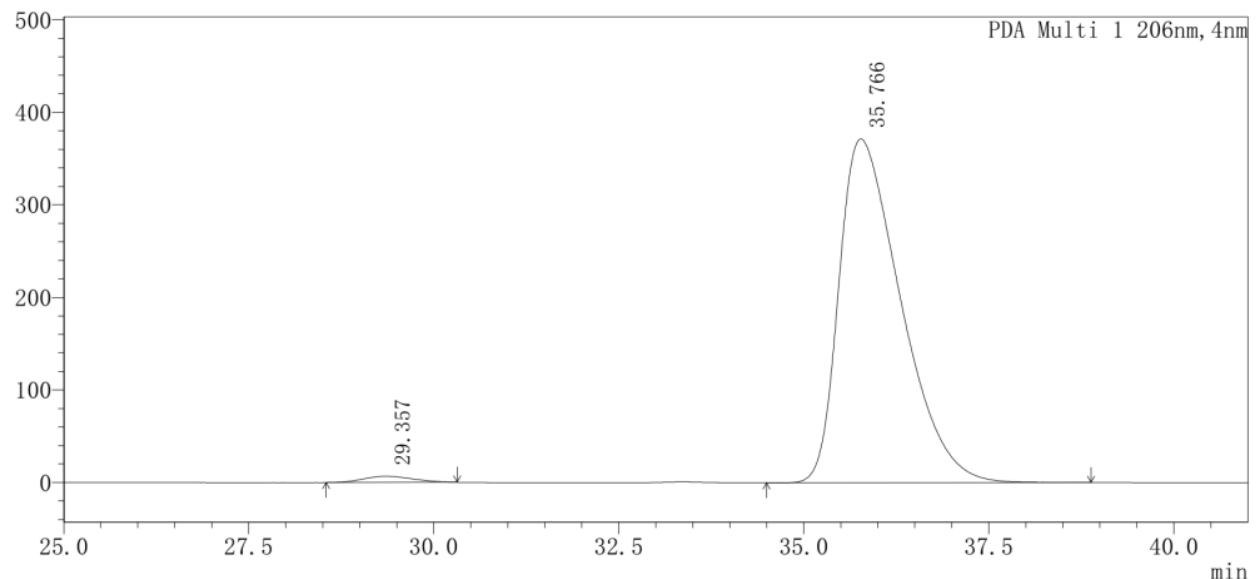

〈Peak Results〉

PDA Ch1 206nm

\begin{tabular}{|c|c|c|c|c|}
\hline Index & Time/min & Height/mAU & Quantity/Area & Area \%/\% \\
\hline 1 & 29.357 & 6702 & 307883 & 1.408 \\
\hline 2 & 35.766 & 371517 & 21552352 & 98.592 \\
\hline
\end{tabular}




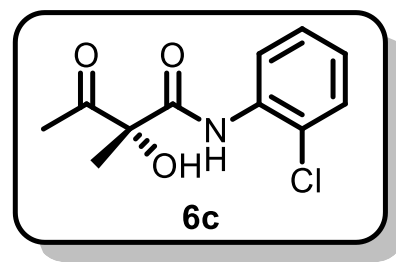

HPLC (ChiralPak AD-H, 5\% i-PrOH in hexanes, $1.0 \mathrm{~mL} / \mathrm{min}, 208 \mathrm{~nm}$ )

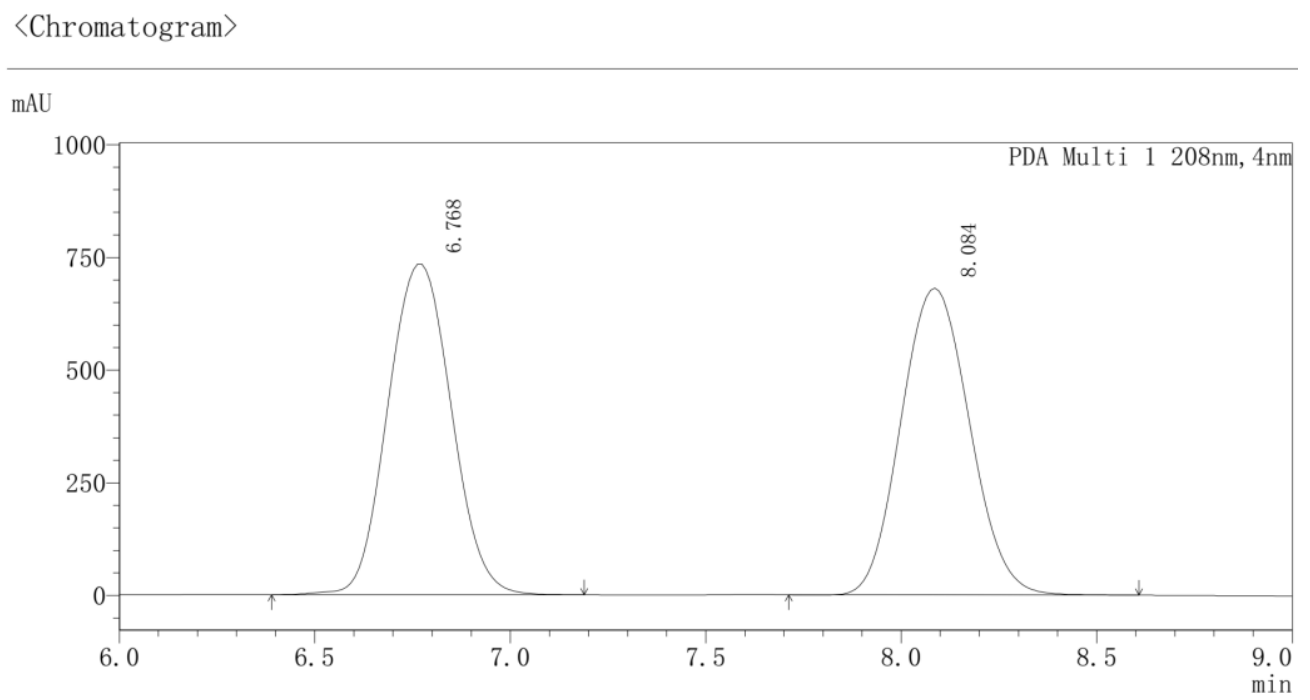

〈Peak Results〉

PDA Ch1 208nm

\begin{tabular}{|c|c|c|c|c|}
\hline Index & Time/min & Height/mAU & Quantity/Area & Area \%/\% \\
\hline 1 & 6.768 & 733472 & 8301432 & 49.949 \\
\hline 2 & 8.084 & 680359 & 8318533 & 50.051 \\
\hline
\end{tabular}

$\langle$ Chromatogram〉

$\mathrm{mAU}$

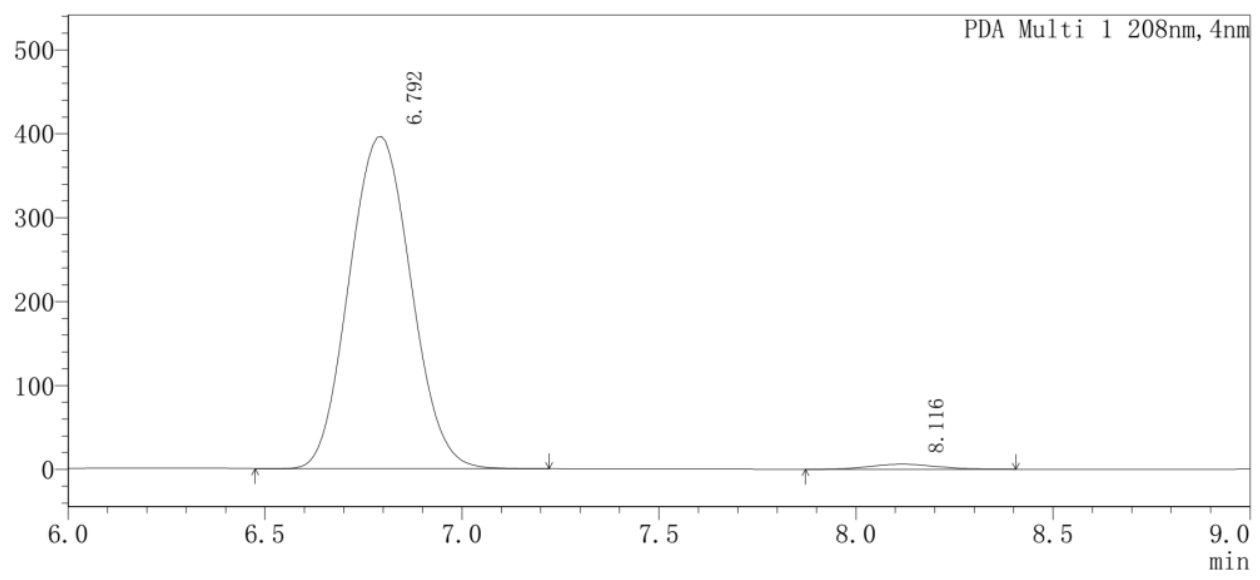

〈Peak Results〉

PDA Ch1 208nm

\begin{tabular}{|c|c|c|c|c|}
\hline Index & Time/min & Height/mAU & Quantity/Area & Area \%/\% \\
\hline 1 & 6.792 & 396330 & 4335318 & 98.421 \\
\hline 2 & 8.116 & 5968 & 69536 & 1.579 \\
\hline
\end{tabular}




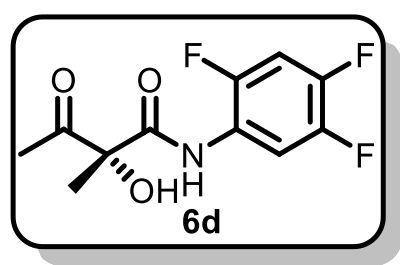

HPLC (ChiralPak OJ-H, 10\% i-PrOH in hexanes, $1.0 \mathrm{~mL} / \mathrm{min}, 239 \mathrm{~nm}$ )

$\langle$ Chromatogram〉

$\mathrm{mAU}$

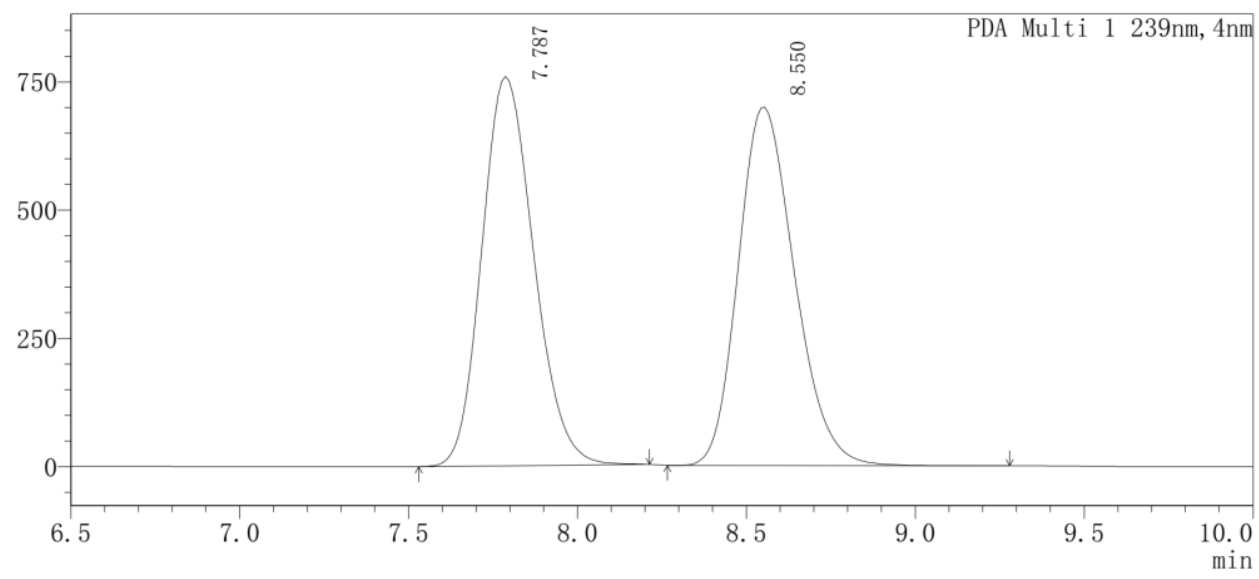

〈Peak Results〉

PDA Ch1 239nm

\begin{tabular}{|c|c|c|c|c|}
\hline Index & Time/min & Height/mAU & Quantity/Area & Area \%/\% \\
\hline 1 & 7.787 & 758905 & 8119799 & 50.095 \\
\hline 2 & 8.550 & 698399 & 8089036 & 49.905 \\
\hline
\end{tabular}

$\langle$ Chromatogram〉

$\mathrm{mAU}$

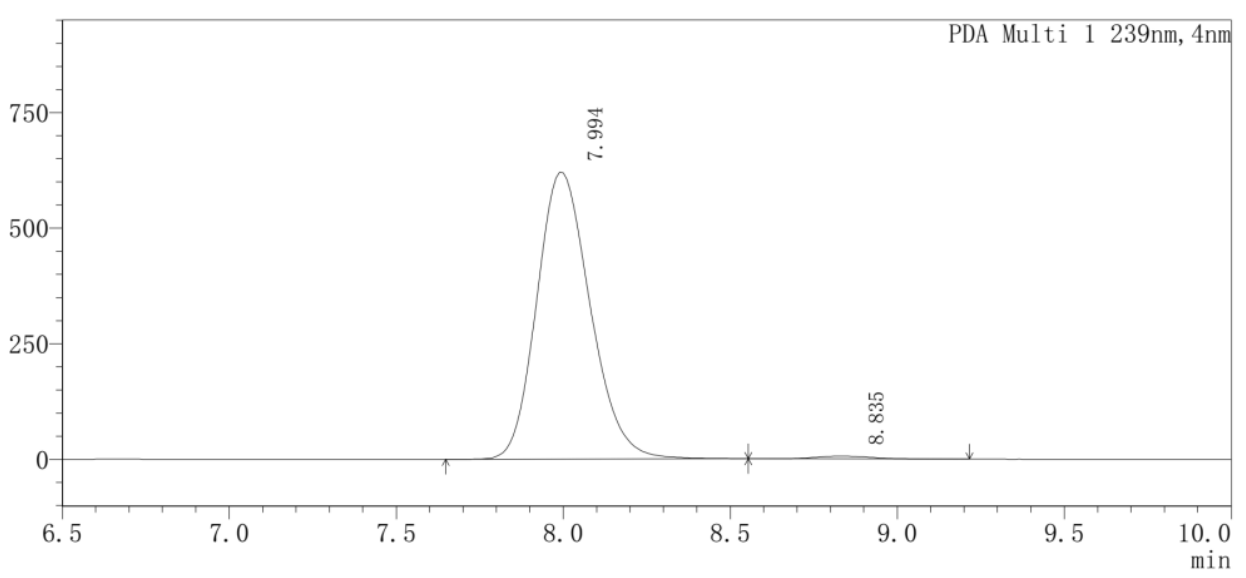

〈Peak Results〉

PDA Ch1 239nm

\begin{tabular}{|c|c|c|c|c|}
\hline Index & Time/min & Height/mAU & Quantity/Area & Area $\% / \%$ \\
\hline 1 & 7.994 & 620824 & 6904901 & 99.029 \\
\hline 2 & 8.835 & 6031 & 67693 & 0.971 \\
\hline
\end{tabular}




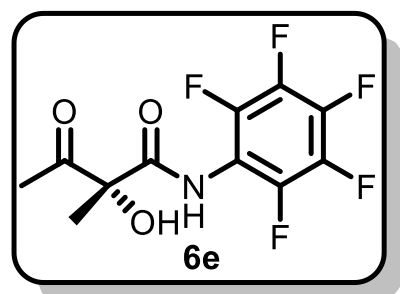

HPLC (ChiralPak OJ-H, 5\% i-PrOH in hexanes, $1.0 \mathrm{~mL} / \mathrm{min}, 219 \mathrm{~nm}$ )

〈Chromatogram〉

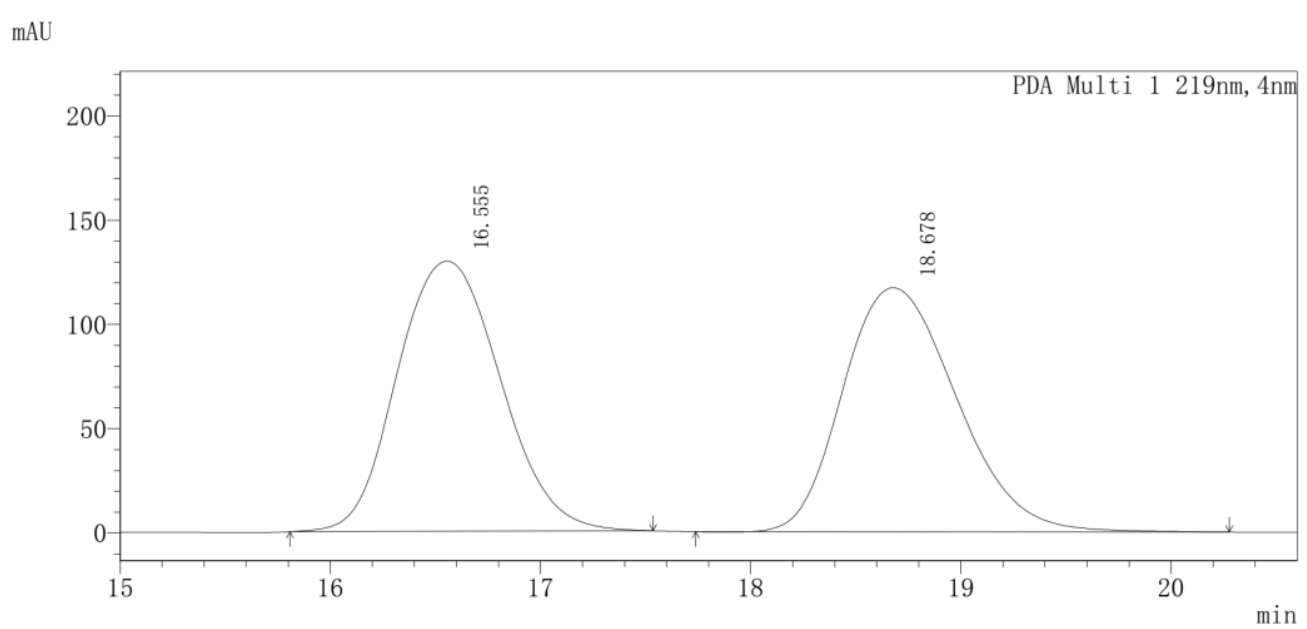

〈Peak Results〉

PDA Ch1 219nm

\begin{tabular}{|c|c|c|c|c|}
\hline Index & Time/min & Height/mAU & Quantity/Area & Area \%/\% \\
\hline 1 & 16.555 & 129606 & 4429861 & 50.105 \\
\hline 2 & 18.678 & 117069 & 4411334 & 49.895 \\
\hline
\end{tabular}

〈Chromatogram〉

mAU

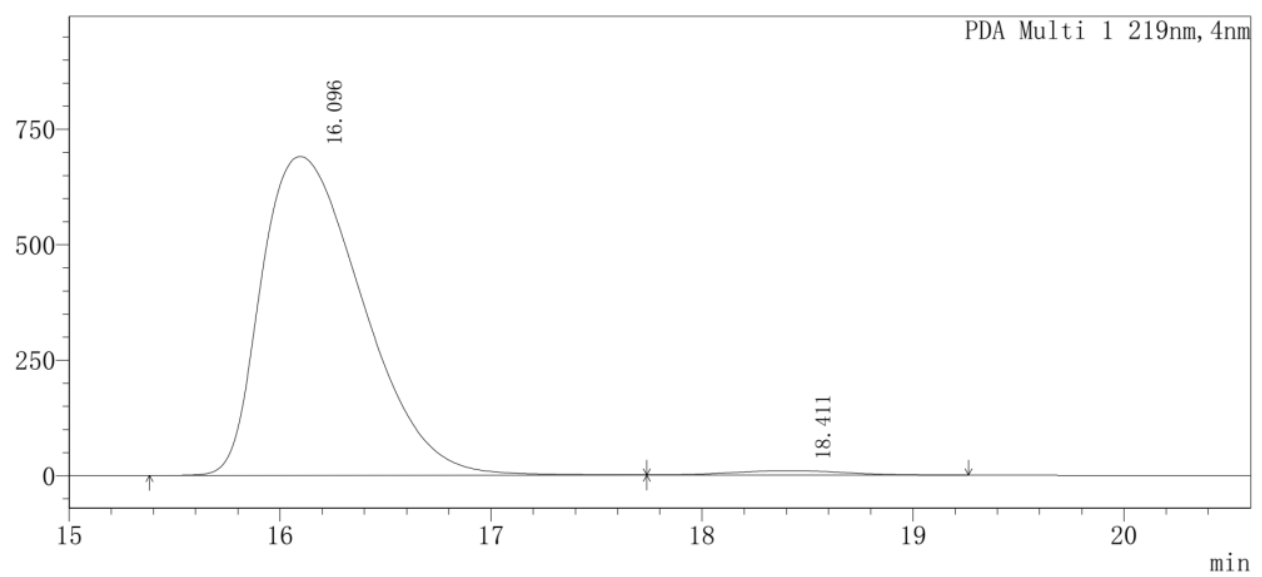

$\langle$ Peak Results〉

PDA Ch1 219nm

\begin{tabular}{|c|c|c|c|c|}
\hline Index & Time/min & Height/mAU & Quantity/Area & Area \%/\% \\
\hline 1 & 16.096 & 690605 & 23040077 & 98.434 \\
\hline 2 & 18.411 & 9689 & 366617 & 1.566 \\
\hline
\end{tabular}




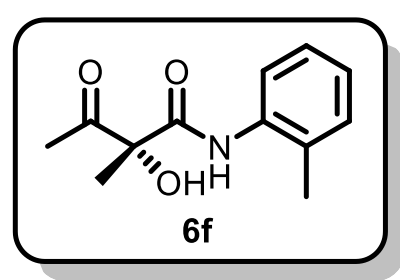

HPLC (ChiralPak OJ-H, 5\% i-PrOH in hexanes, $1.2 \mathrm{~mL} / \mathrm{min}, 206 \mathrm{~nm}$ )

$\langle$ Chromatogram〉

$\mathrm{mAU}$

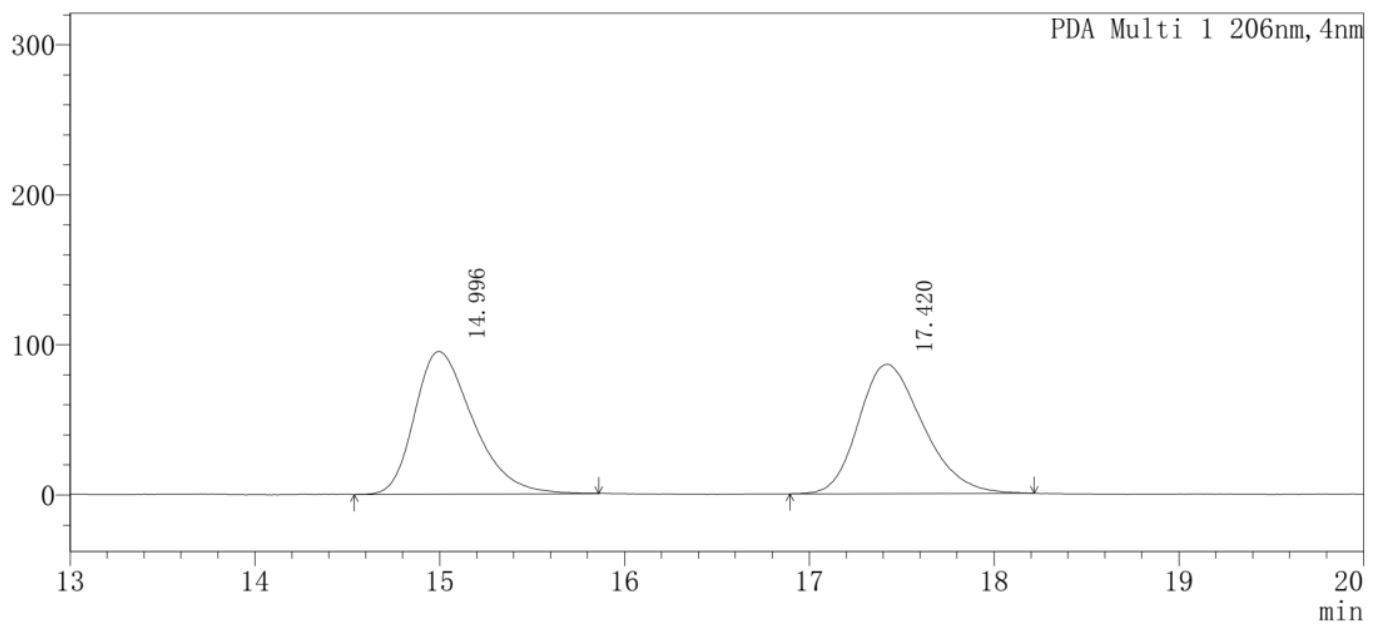

〈Peak Results〉

PDA Ch1 206nm

\begin{tabular}{|c|c|c|c|c|}
\hline Index & Time/min & Height/mAU & Quantity/Area & Area \%/\% \\
\hline 1 & 14.996 & 95082 & 2088743 & 49.974 \\
\hline 2 & 17.420 & 86277 & 2090928 & 50.026 \\
\hline
\end{tabular}

〈Chromatogram〉

$\mathrm{mAU}$

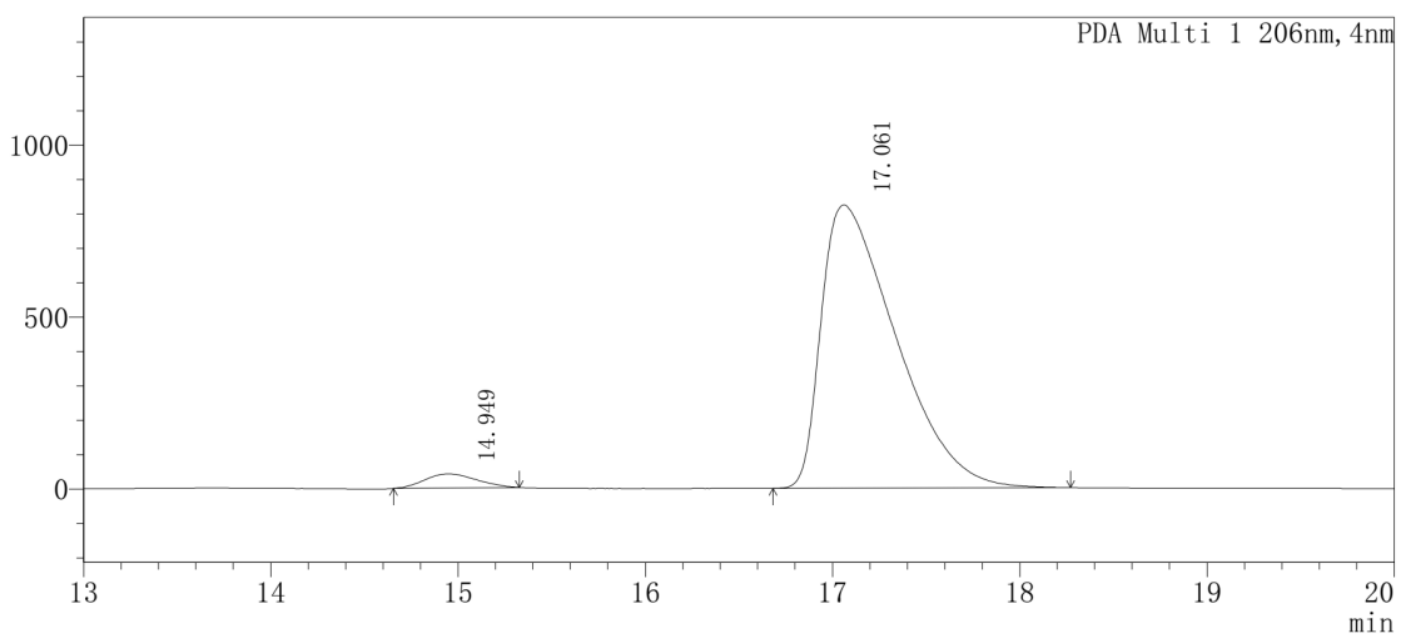

〈Peak Results〉

PDA Ch1 206nm

\begin{tabular}{|c|c|c|c|c|}
\hline Index & Time/min & Height/mAU & Quantity/Area & Area \%/\% \\
\hline 1 & 14.949 & 41124 & 792103 & 3.353 \\
\hline 2 & 17.061 & 823404 & 22828722 & 96.647 \\
\hline
\end{tabular}




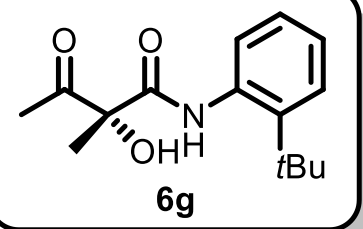

HPLC (ChiralPak OJ-H, 5\% i-PrOH in hexanes, $1.0 \mathrm{~mL} / \mathrm{min}, 206 \mathrm{~nm}$ )

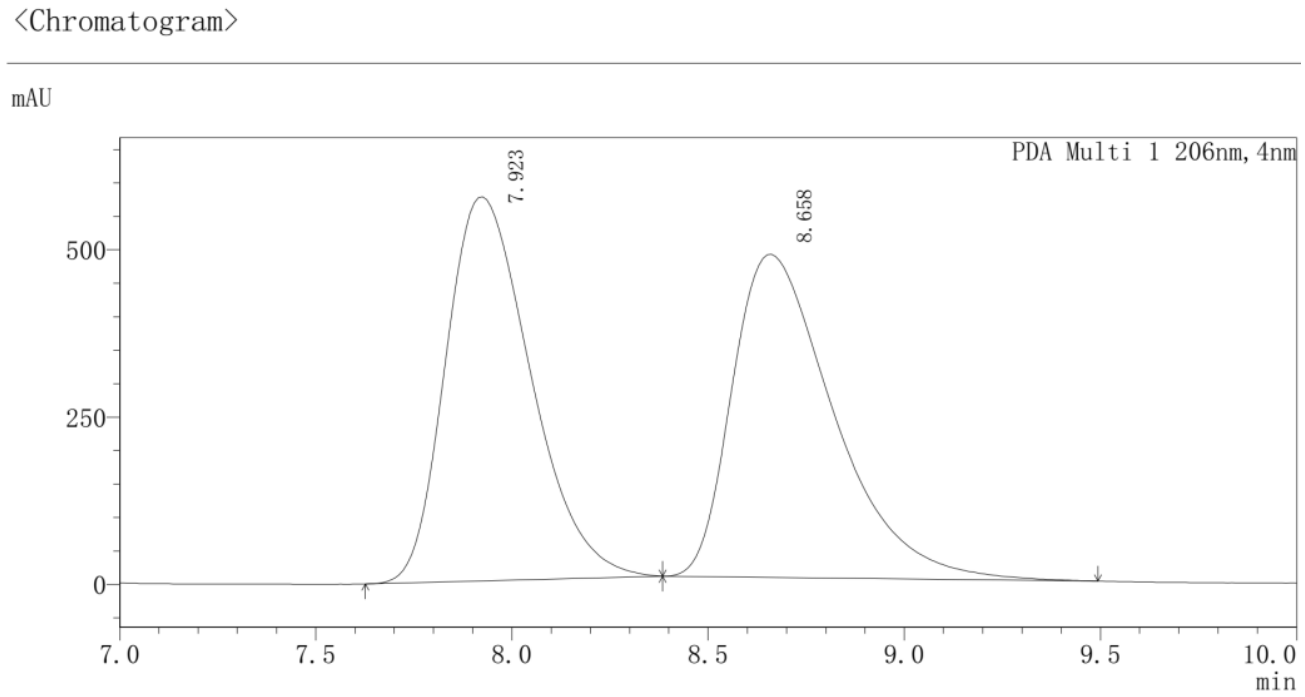

〈Peak Results〉

PDA Ch1 206nm

\begin{tabular}{|c|c|c|c|c|}
\hline Index & Time/min & Height/mAU & Quantity/Area & Area \%/\% \\
\hline 1 & 7.923 & 574112 & 8651749 & 49.843 \\
\hline 2 & 8.658 & 482791 & 8706177 & 50.157 \\
\hline
\end{tabular}

〈Chromatogram〉

mAU

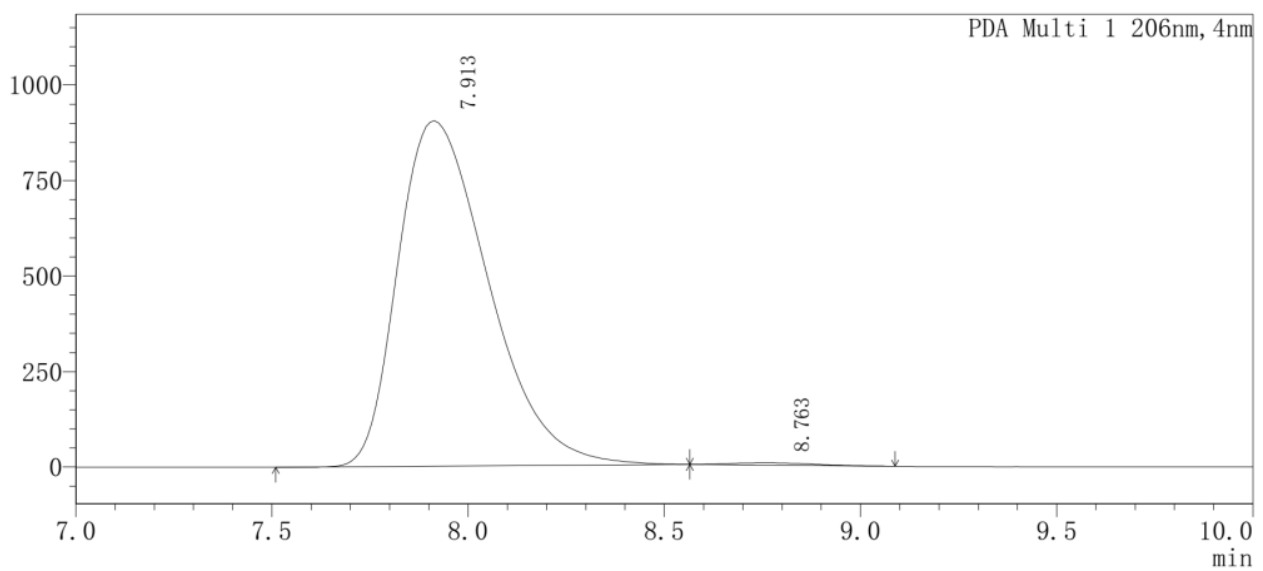

〈Peak Results〉

PDA Ch1 206nm

\begin{tabular}{|c|c|c|c|c|}
\hline Index & Time/min & Height/mAU & Quantity/Area & Area \%/\% \\
\hline 1 & 7.913 & 904076 & 14532738 & 99.411 \\
\hline 2 & 8.763 & 5895 & 86070 & 0.589 \\
\hline
\end{tabular}




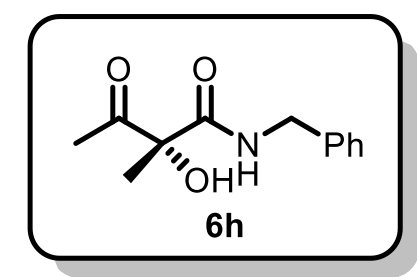

HPLC (ChiralPak OJ-H, 10\% i-PrOH in hexanes, $1.0 \mathrm{~mL} / \mathrm{min}, 209 \mathrm{~nm}$ )

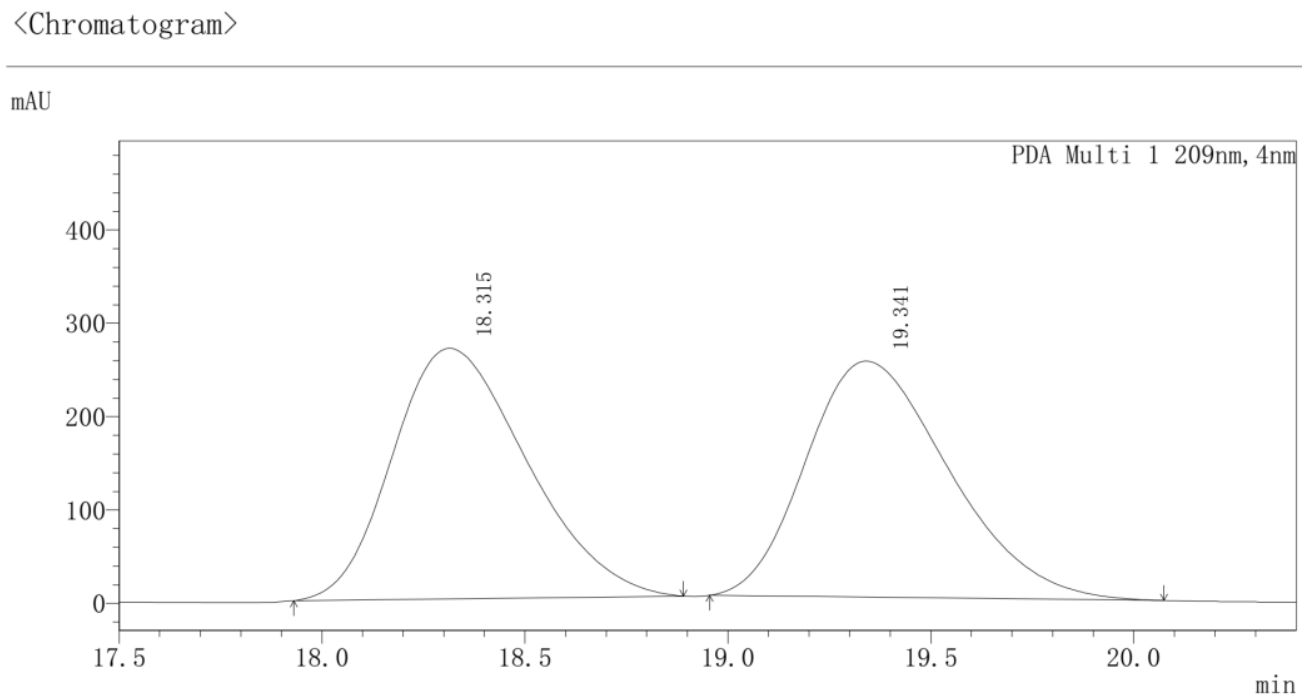

〈Peak Results >

PDA Ch1 209nm

\begin{tabular}{|c|c|c|c|c|}
\hline Index & Time/min & Height/mAU & Quantity/Area & Area \%/\% \\
\hline 1 & 18.315 & 268524 & 6177085 & 49.871 \\
\hline 2 & 19.341 & 252943 & 6208931 & 50.129 \\
\hline
\end{tabular}

$\langle$ Chromatogram〉

$\mathrm{mAU}$

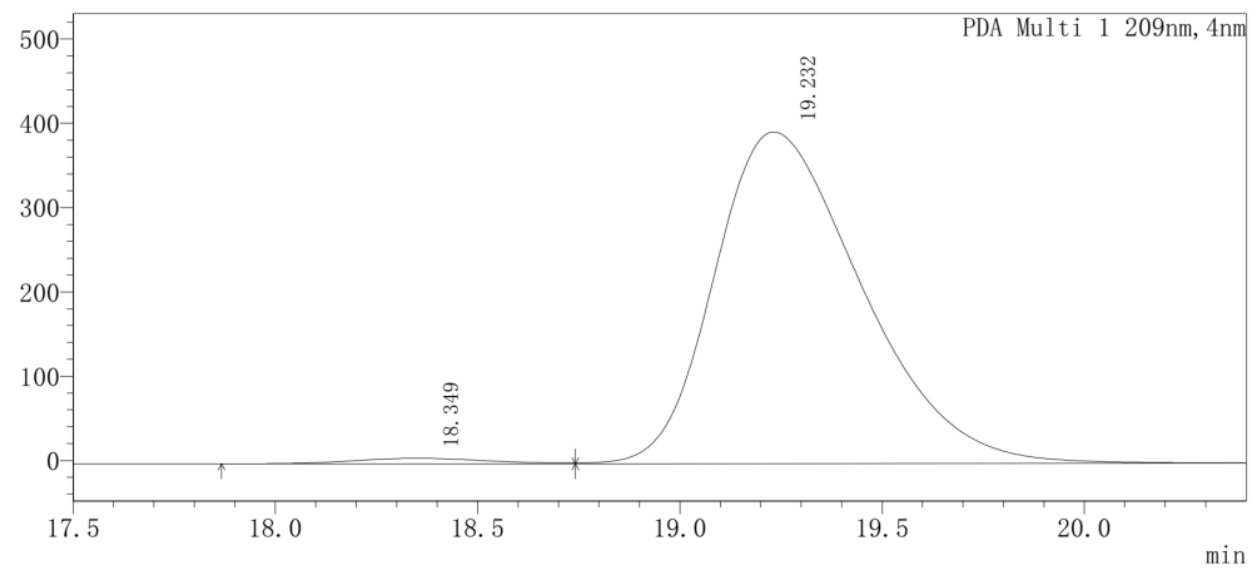

〈Peak Results>

PDA Ch1 209nm

\begin{tabular}{|c|c|c|c|c|}
\hline Index & Time/min & Height/mAU & Quantity/Area & Area \%/\% \\
\hline 1 & 18.349 & 6476 & 148868 & 1.468 \\
\hline 2 & 19.232 & 393583 & 9989205 & 98.532 \\
\hline
\end{tabular}




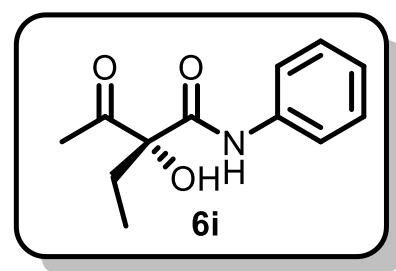

HPLC (ChiralPak AD-H, 2\% i-PrOH in hexanes, $0.8 \mathrm{~mL} / \mathrm{min}, 246 \mathrm{~nm}$ )

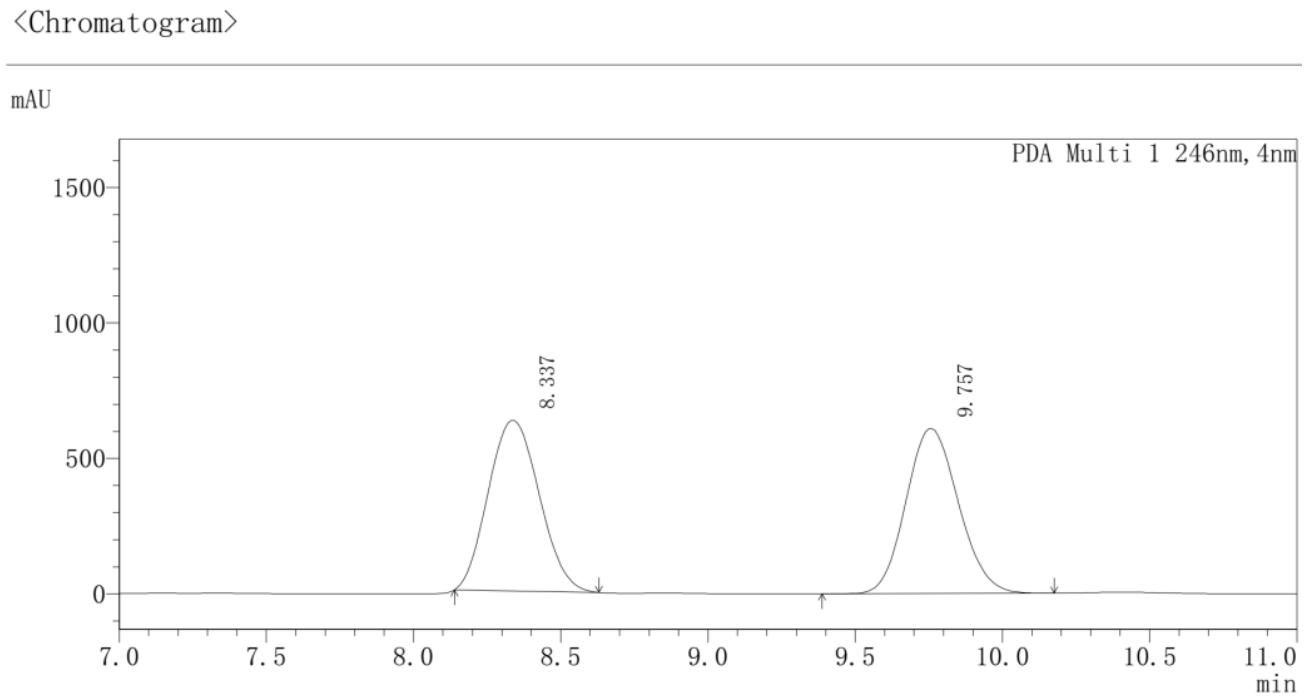

〈Peak Results

PDA Ch1 246nm

\begin{tabular}{|c|c|c|c|c|}
\hline Index & Time/min & Height/mAU & Quantity/Area & Area \%/\% \\
\hline 1 & 8.337 & 630021 & 7592679 & 50.113 \\
\hline 2 & 9.757 & 609434 & 7558535 & 49.887 \\
\hline
\end{tabular}

〈Chromatogram〉

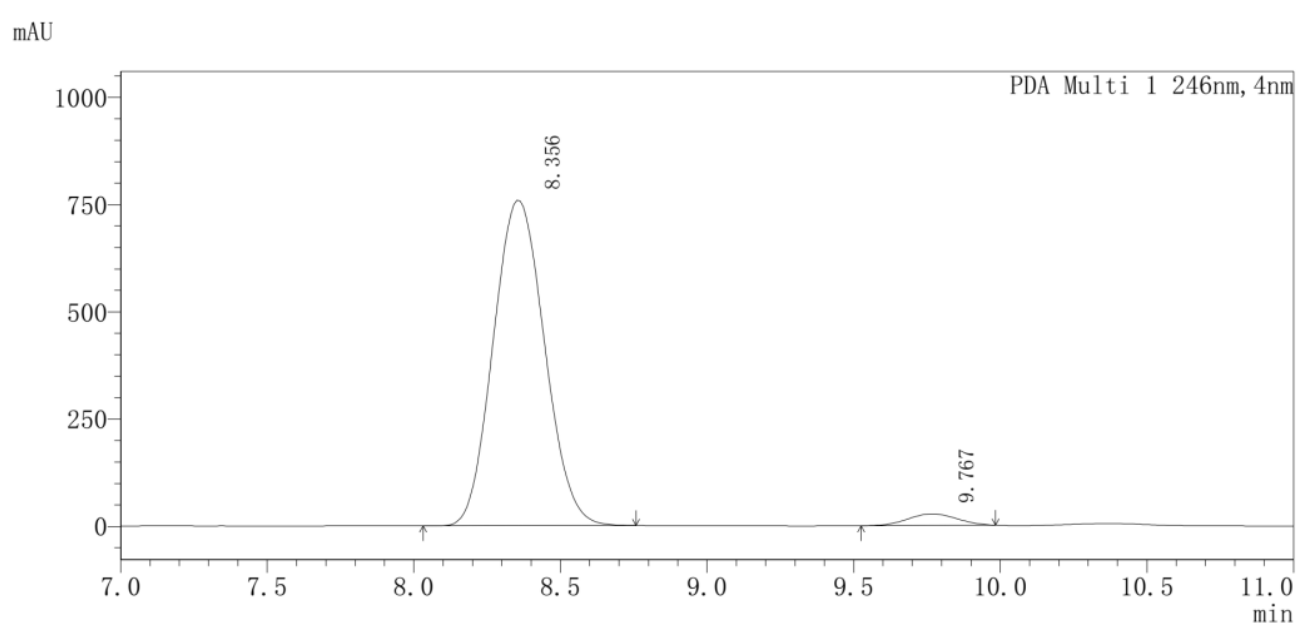

〈Peak Results〉

PDA Ch1 246nm

\begin{tabular}{|c|c|c|c|c|}
\hline Index & Time/min & Height/mAU & Quantity/Area & Area \%/\% \\
\hline 1 & 8.356 & 758590 & 9236923 & 96.664 \\
\hline 2 & 9.767 & 27202 & 318729 & 3.336 \\
\hline
\end{tabular}




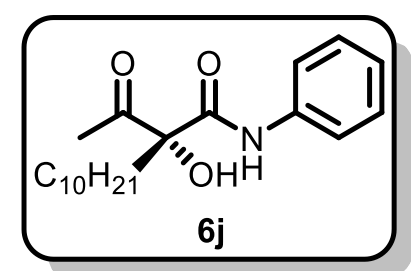

HPLC (ChiralPak AD-H, 2\% i-PrOH in hexanes, $0.8 \mathrm{~mL} / \mathrm{min}, 245 \mathrm{~nm}$ )

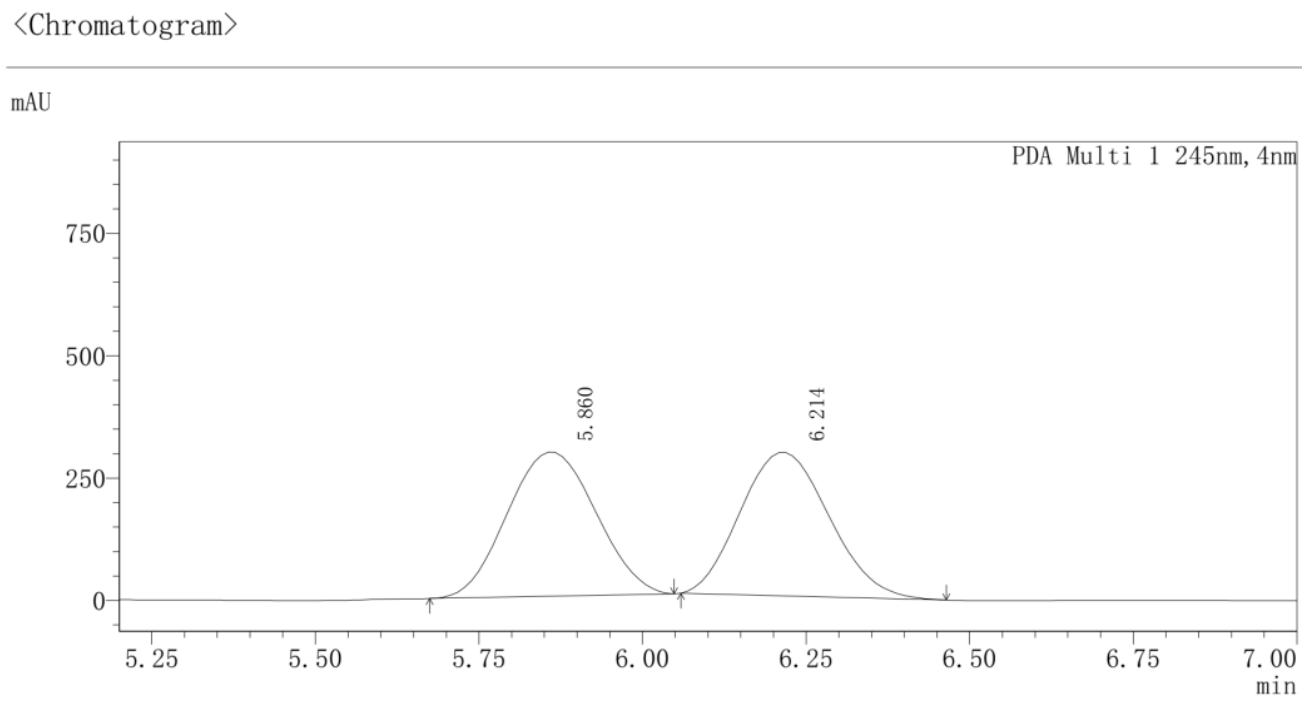

〈Peak Results〉

PDA Ch1 245nm

\begin{tabular}{|c|c|c|c|c|}
\hline Index & Time/min & Height/mAU & Quantity/Area & Area $\% / \%$ \\
\hline 1 & 5.860 & 294582 & 2781521 & 50.136 \\
\hline 2 & 6.214 & 292792 & 2766403 & 49.864 \\
\hline
\end{tabular}

〈Chromatogram〉

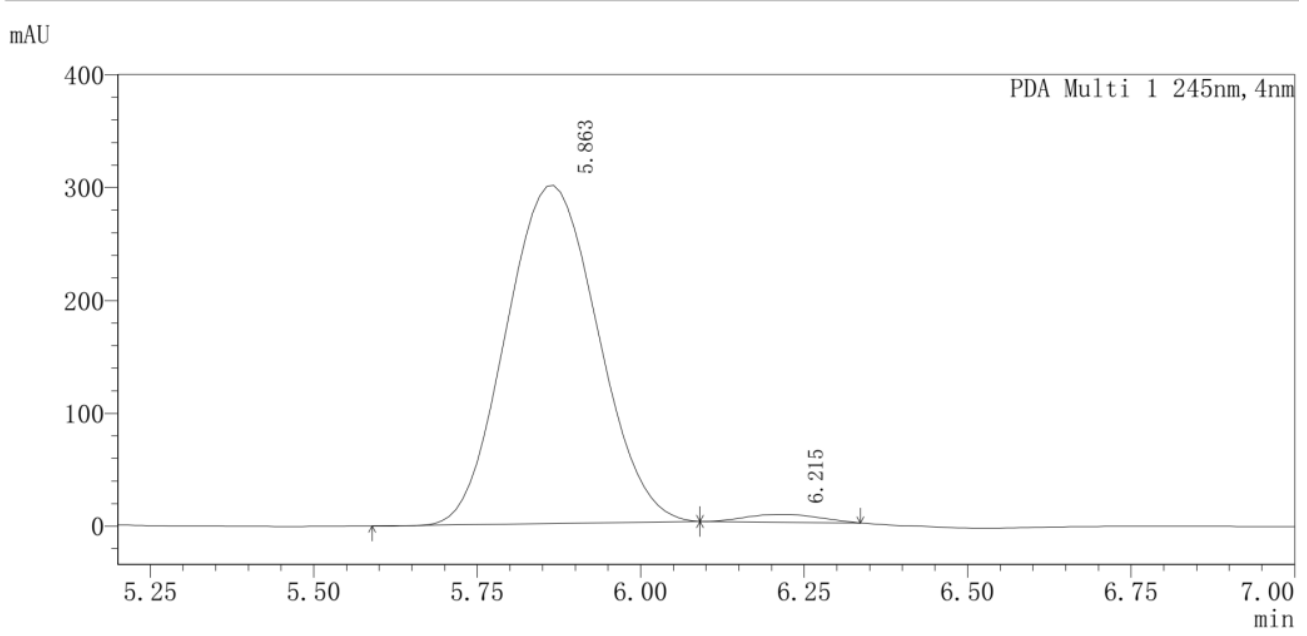

〈Peak Results>

PDA Ch1 245nm

\begin{tabular}{|c|c|c|c|c|}
\hline Index & Time/min & Height/mAU & Quantity/Area & Area \%/\% \\
\hline 1 & 5.863 & 299697 & 2925886 & 98.173 \\
\hline 2 & 6.215 & 7077 & 54452 & 1.827 \\
\hline
\end{tabular}




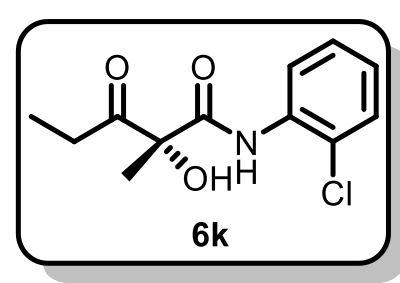

HPLC (ChiralPak AD-H, 2\% i-PrOH in hexanes, $0.8 \mathrm{~mL} / \mathrm{min}, 245 \mathrm{~nm}$ )

$\langle$ Chromatogram〉

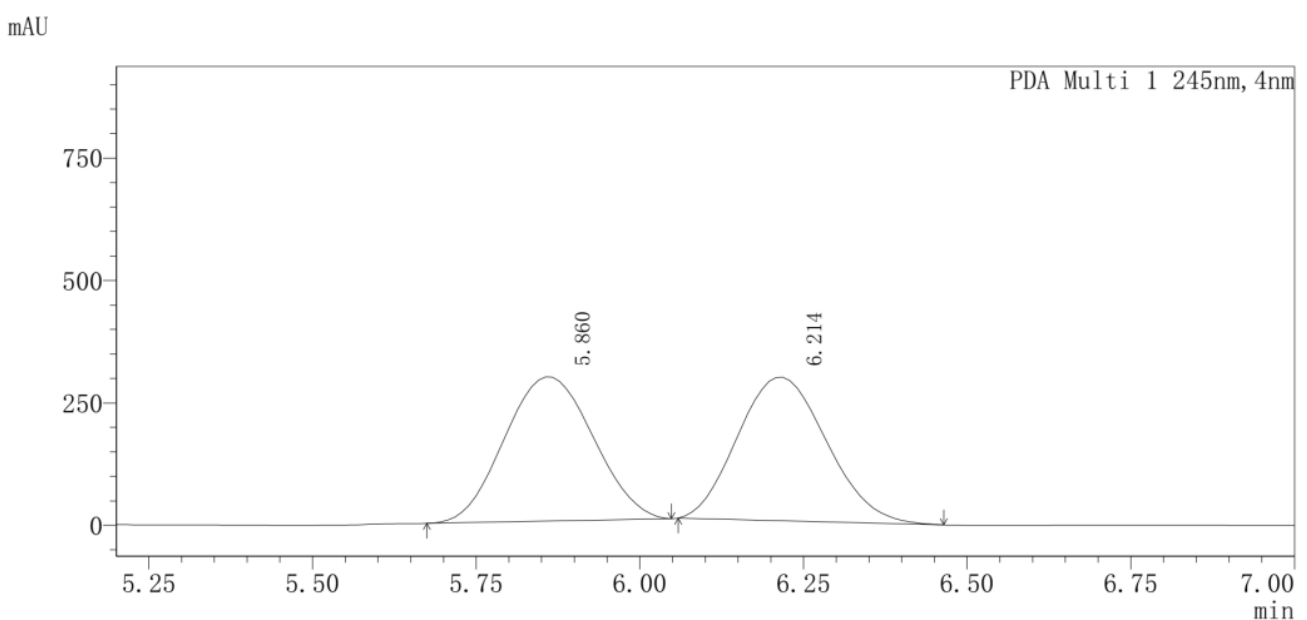

〈Peak Results〉

PDA Ch1 245nm

\begin{tabular}{|c|c|c|c|c|}
\hline Index & Time/min & Height/mAU & Quantity/Area & Area \%/\% \\
\hline 1 & 5.860 & 294582 & 2781521 & 50.136 \\
\hline 2 & 6.214 & 292792 & 2766403 & 49.864 \\
\hline
\end{tabular}

$\langle$ Chromatogram〉

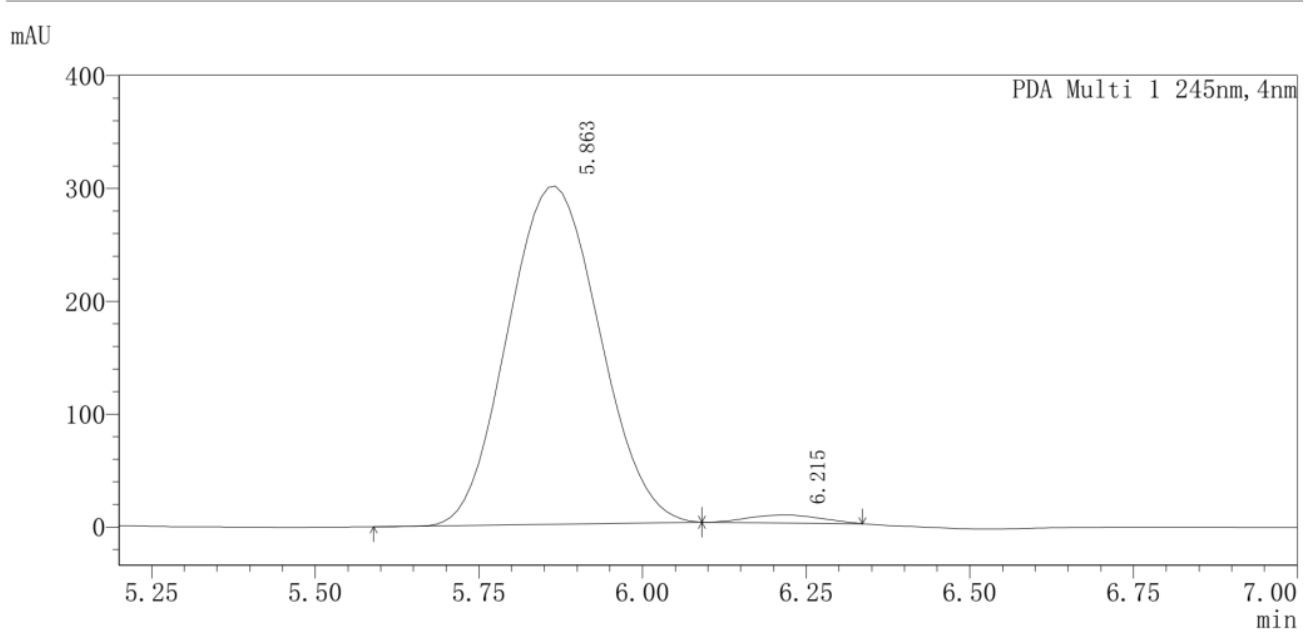

$\langle$ Peak Results〉

PDA Ch1 245nm

\begin{tabular}{|c|c|c|c|c|}
\hline Index & Time/min & Height/mAU & Quantity/Area & Area \%/\% \\
\hline 1 & 5.863 & 299697 & 2925886 & 98.173 \\
\hline 2 & 6.215 & 7077 & 54452 & 1.827 \\
\hline
\end{tabular}




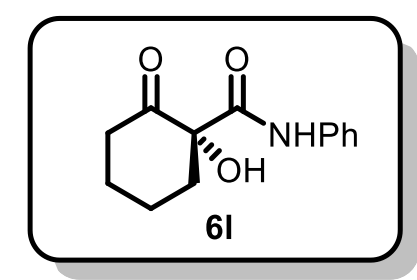

HPLC (ChiralPak OJ-H, 10\% i-PrOH in hexanes, $1.0 \mathrm{~mL} / \mathrm{min}, 244 \mathrm{~nm}$ )

$\langle$ Chromatogram〉

$\mathrm{mAU}$

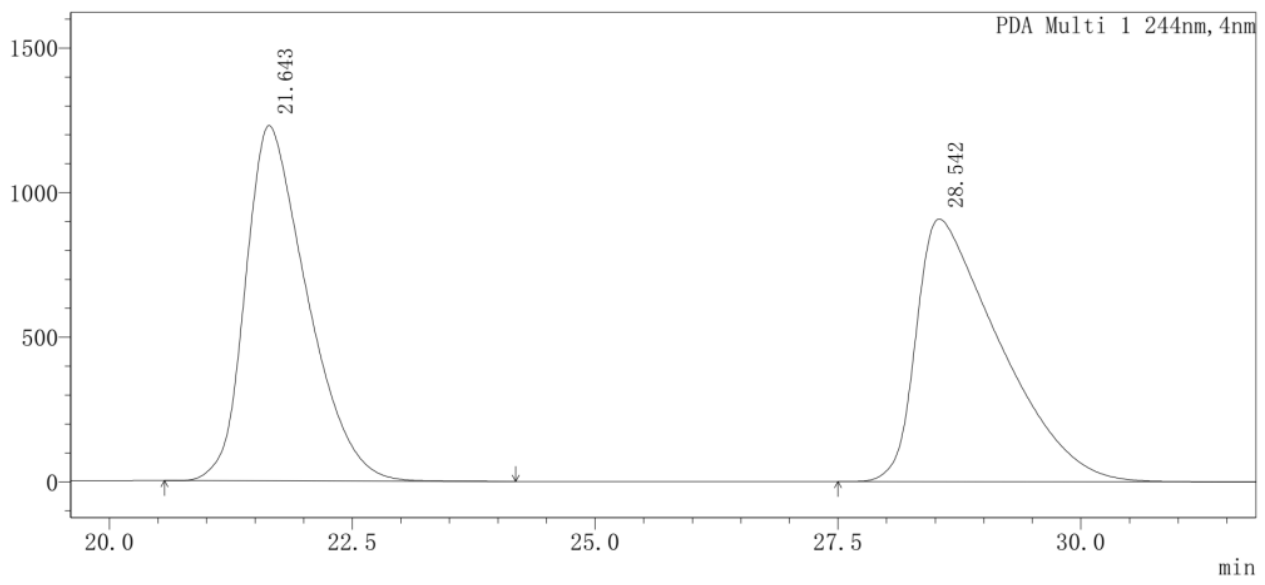

〈Peak Results〉

PDA Ch1 244nm

\begin{tabular}{|c|c|c|c|c|} 
PDA Chl & \multicolumn{1}{|c|}{ Anm } & & \\
\hline Index & Time/min & Height/mAU & Quantity/Area & Area $\% / \%$ \\
\hline 1 & 21.643 & 1229067 & 54293151 & 49.969 \\
\hline 2 & 28.542 & 907892 & 54359825 & 50.031 \\
\hline
\end{tabular}

$\langle$ Chromatogram〉

$\mathrm{mAU}$

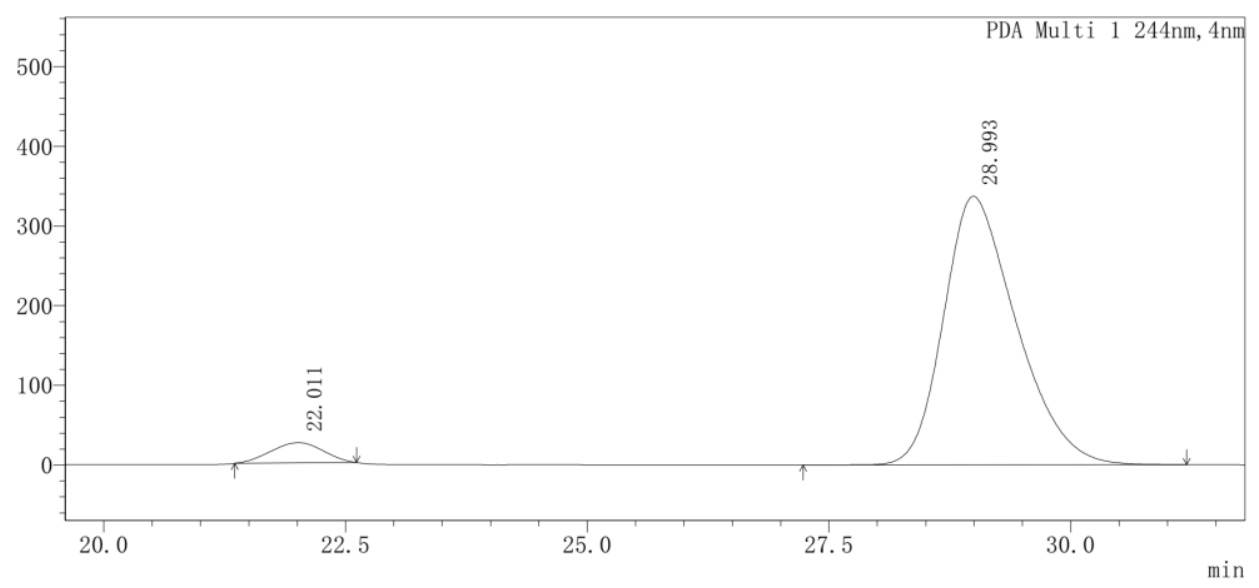

〈Peak Results〉

PDA Ch1 244nm

\begin{tabular}{|c|c|c|c|c|}
\hline Index & Time/min & Height/mAU & Quantity/Area & Area \%/\% \\
\hline 1 & 22.011 & 25469 & 958803 & 5.155 \\
\hline 2 & 28.993 & 336969 & 17640050 & 94.845 \\
\hline
\end{tabular}




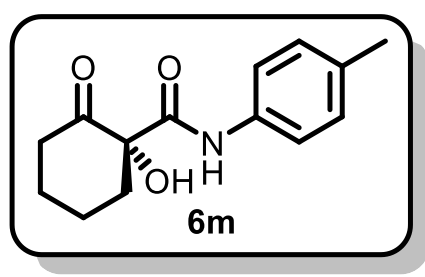

HPLC (ChiralPak OJ-H, 10\% i-PrOH in hexanes, $1.0 \mathrm{~mL} / \mathrm{min}, 207 \mathrm{~nm}$ )

$\langle$ Chromatogram〉

$\mathrm{mAU}$

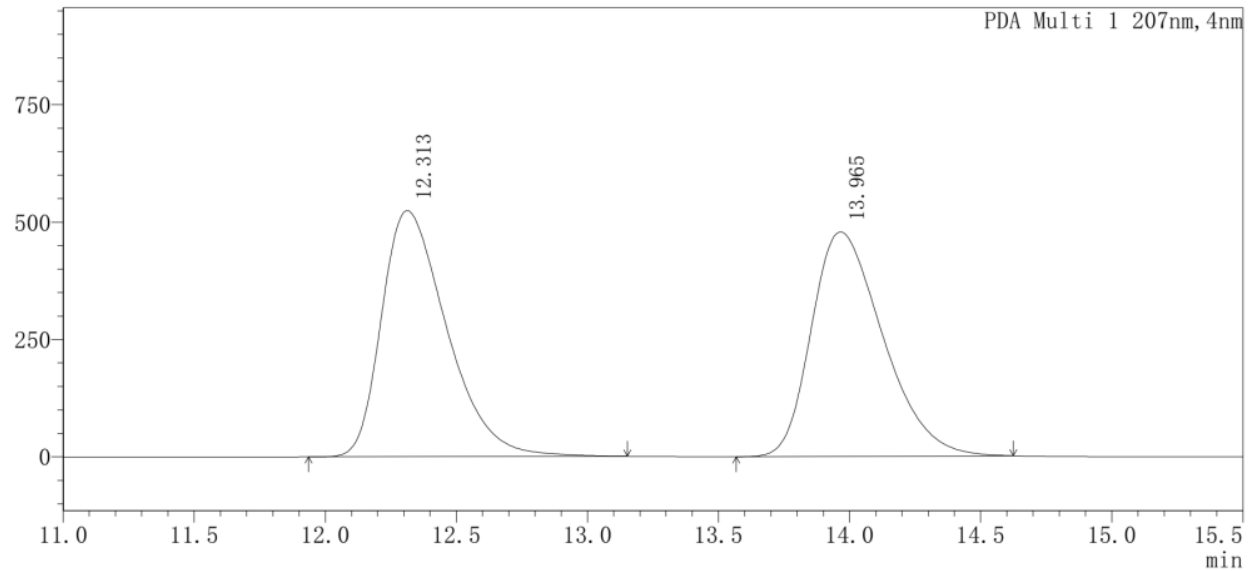

〈Peak Results

PDA Ch1 207nm

\begin{tabular}{|c|c|c|c|c|}
\hline Index & Time/min & Height/mAU & Quantity/Area & Area \%/\% \\
\hline 1 & 12.313 & 524239 & 9086916 & 50.004 \\
\hline 2 & 13.965 & 478044 & 9085486 & 49.996 \\
\hline
\end{tabular}

〈Chromatogram〉

$\mathrm{mAU}$

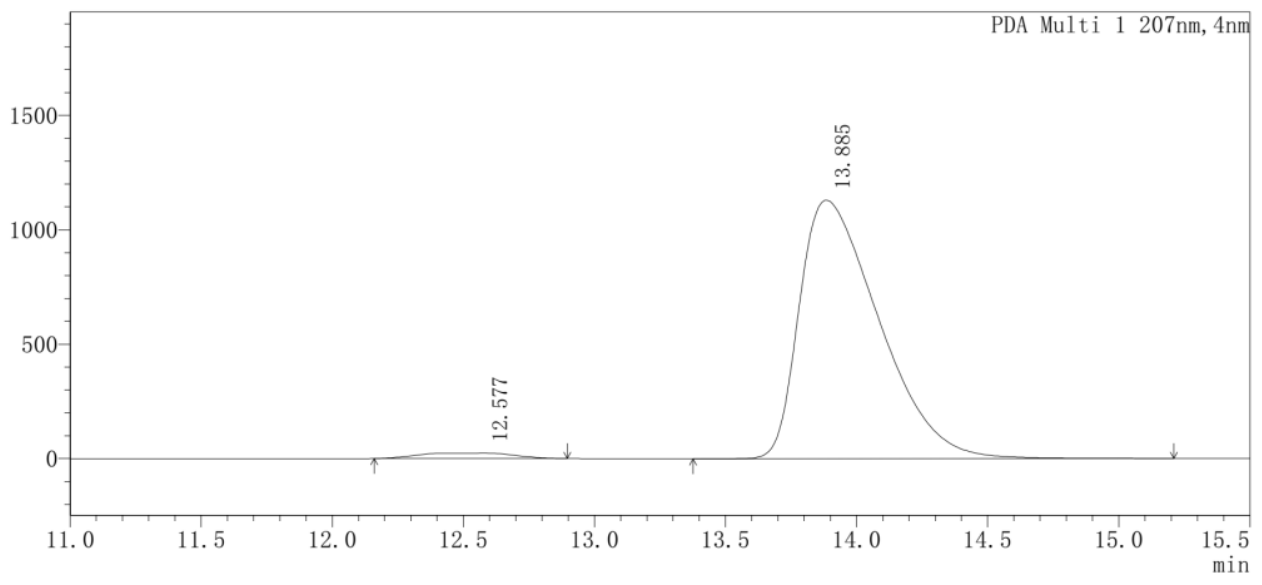

〈Peak Results

PDA Ch1 207nm

\begin{tabular}{|c|c|c|c|c|}
\hline Index & Time/min & Height/mAU & Quantity/Area & Area \%/\% \\
\hline 1 & 12.577 & 23774 & 601249 & 2.456 \\
\hline 2 & 13.885 & 1129682 & 23878630 & 97.544 \\
\hline
\end{tabular}




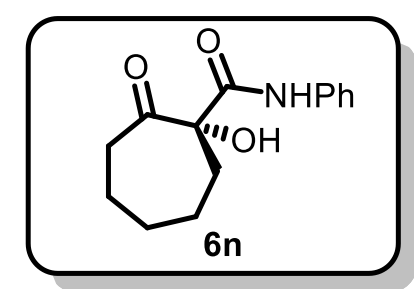

HPLC (ChiralPak OJ-H, 5\% i-PrOH in hexanes, $1.0 \mathrm{~mL} / \mathrm{min}, 245 \mathrm{~nm}$ )

$\langle$ Chromatogram〉

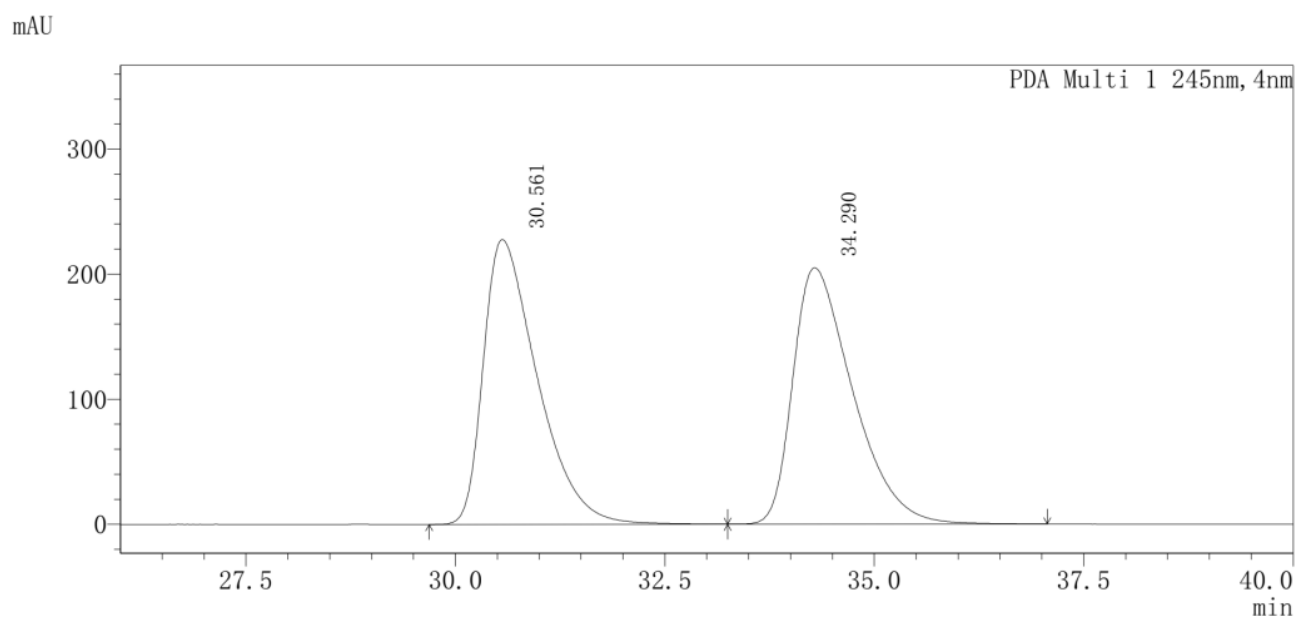

〈Peak Results〉

PDA Ch1 245nm

\begin{tabular}{|c|c|c|c|c|}
\hline Index & Time/min & Height/mAU & Quantity/Area & Area \%/\% \\
\hline 1 & 30.561 & 227694 & 10208915 & 50.041 \\
\hline 2 & 34.290 & 204949 & 10192010 & 49.959 \\
\hline
\end{tabular}

〈Chromatogram〉

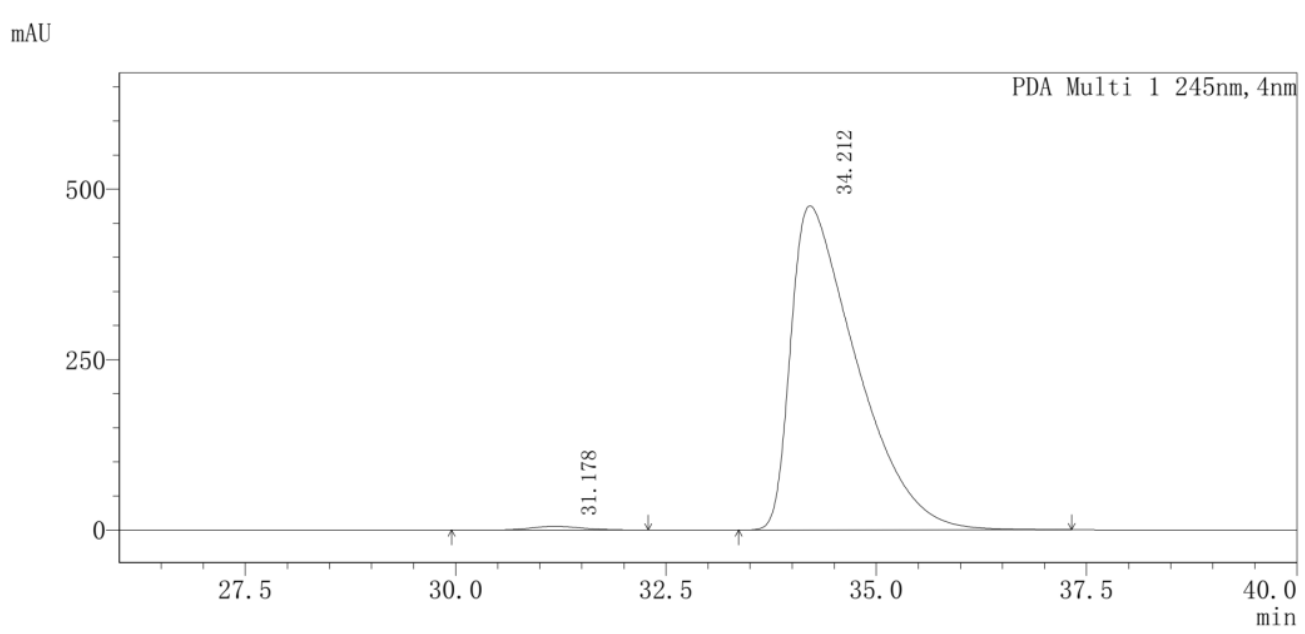

$\langle$ Peak Results〉

PDA Ch1 245nm

\begin{tabular}{|c|c|c|c|c|}
\hline Index & Time/min & Height/mAU & Quantity/Area & Area \%/\% \\
\hline 1 & 31.178 & 5374 & 227922 & 0.872 \\
\hline 2 & 34.212 & 475509 & 25910167 & 99.128 \\
\hline
\end{tabular}




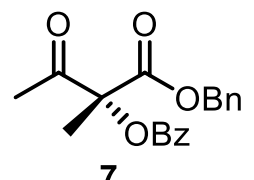

HPLC (ChiralPak OJ-H, 5\% i-PrOH in hexanes, $1.0 \mathrm{~mL} / \mathrm{min}, 230 \mathrm{~nm}$ )

$\langle$ Chromatogram〉

$\mathrm{mAU}$

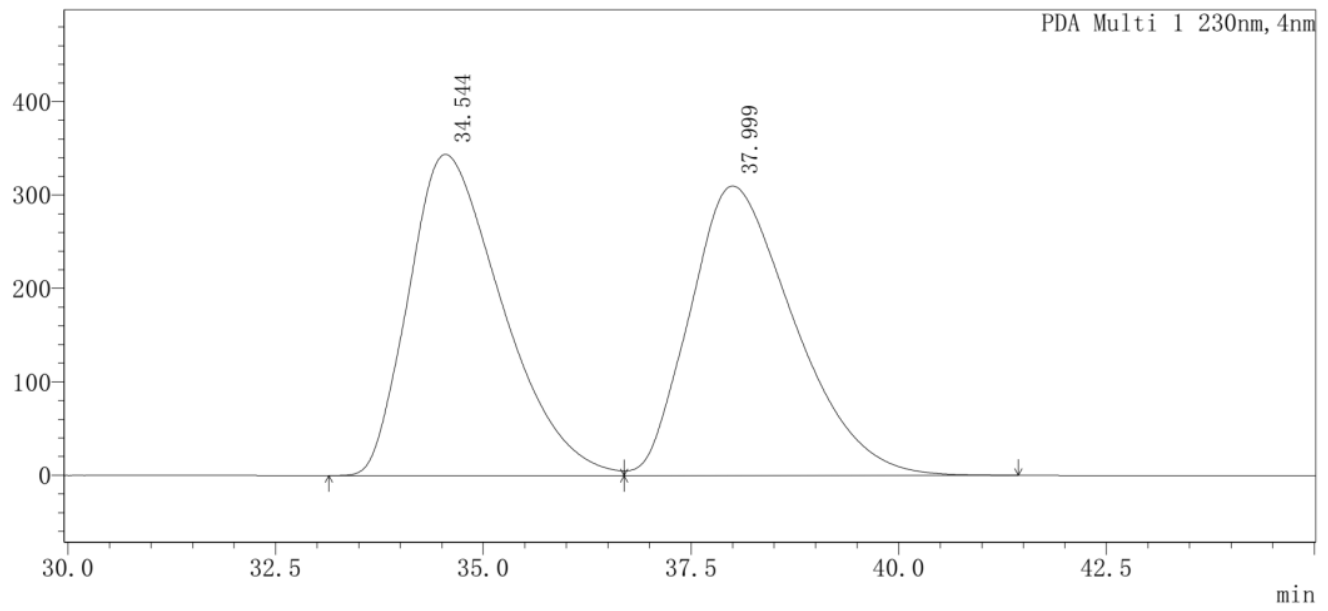

〈Peak Results〉

PDA Ch1 230nm

\begin{tabular}{|c|c|c|c|c|}
\hline Index & Time/min & Height/mAU & Quantity/Area & Area \%/\% \\
\hline 1 & 34.544 & 343876 & 26908650 & 49.868 \\
\hline 2 & 37.999 & 309939 & 27050683 & 50.132 \\
\hline
\end{tabular}

〈Chromatogram〉

$\mathrm{mAU}$

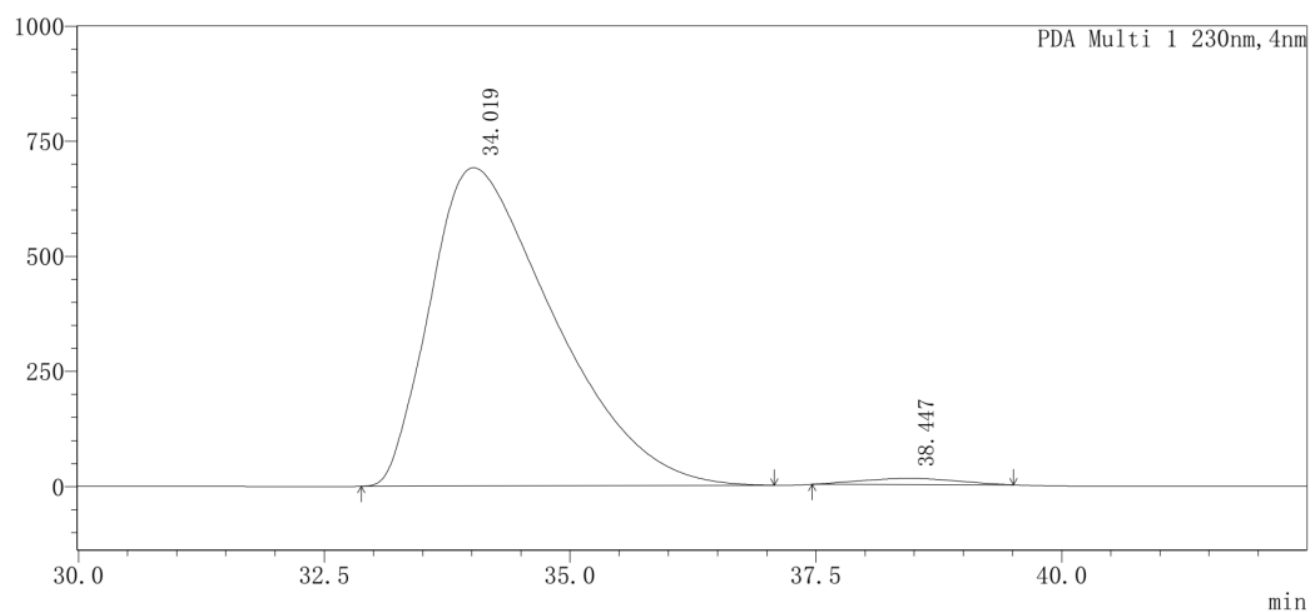

〈Peak Results〉

PDA Ch1 230nm

\begin{tabular}{|c|c|c|c|c|}
\hline Index & Time/min & Height/mAU & Quantity/Area & Area $\% / \%$ \\
\hline 1 & 34.019 & 691537 & 60641725 & 98.538 \\
\hline 2 & 38.447 & 13191 & 899502 & 1.462 \\
\hline
\end{tabular}




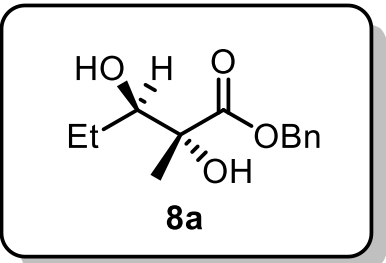

HPLC (ChiralPak AD-H, 5\% i-PrOH in hexanes, $0.8 \mathrm{~mL} / \mathrm{min}, 208 \mathrm{~nm}$ )

〈Chromatogram〉

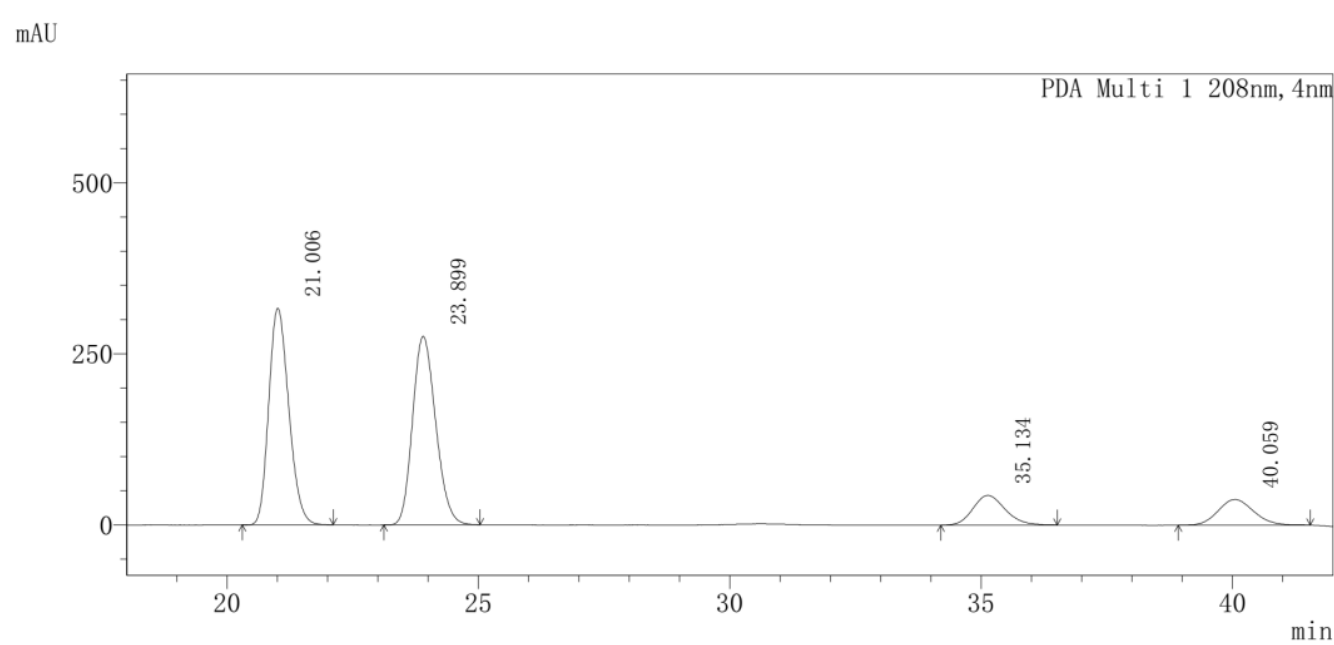

〈Peak Results〉

PDA Ch1 208nm

\begin{tabular}{|c|c|c|c|c|}
\hline Index & Time/min & Height/mAU & Quantity/Area & Area \%/\% \\
\hline 1 & 21.006 & 316585 & 8775009 & 41.058 \\
\hline 2 & 23.899 & 275534 & 8820082 & 41.269 \\
\hline 3 & 35.134 & 43328 & 1925971 & 9.012 \\
\hline 4 & 40.059 & 37646 & 1850967 & 8.661 \\
\hline
\end{tabular}

$\langle$ Chromatogram〉

$\mathrm{mAU}$

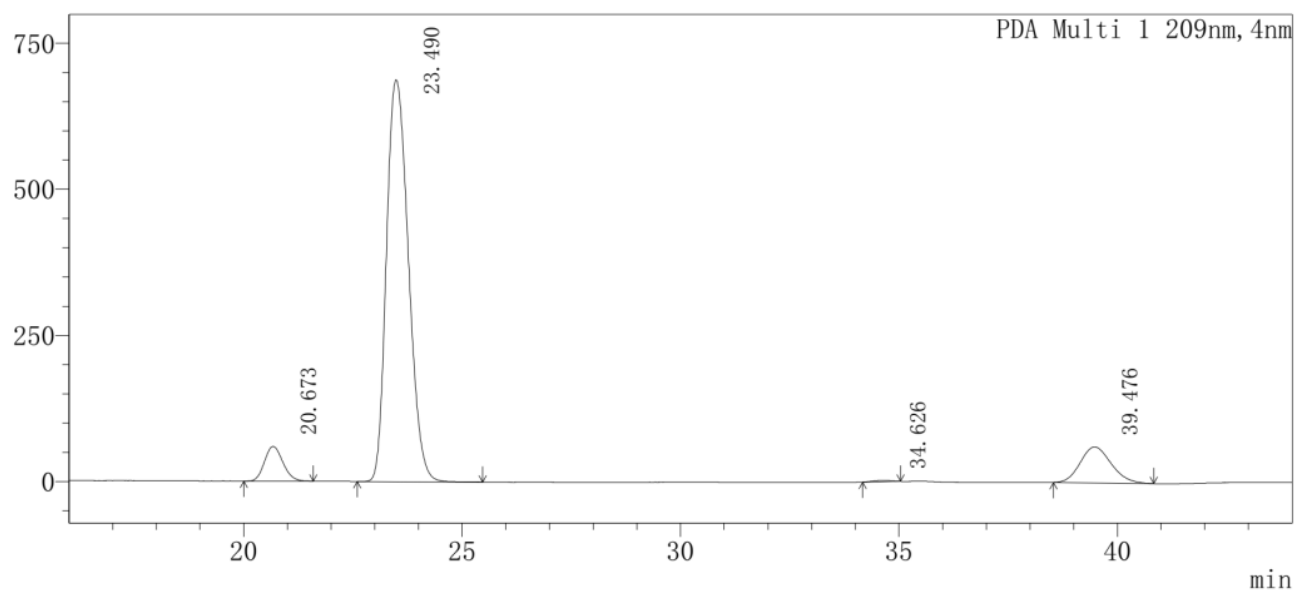

〈Peak Results

PDA Ch1 209nm

\begin{tabular}{|c|c|c|c|c|}
\hline Index & Time/min & Height/mAU & Quantity/Area & Area \%/\% \\
\hline 1 & 20.673 & 59350 & 1735847 & 6.021 \\
\hline 2 & 23.490 & 687419 & 23991447 & 83.211 \\
\hline 3 & 34.626 & 2062 & 60368 & 0.209 \\
\hline 4 & 39.476 & 61637 & 3044513 & 10.559 \\
\hline
\end{tabular}




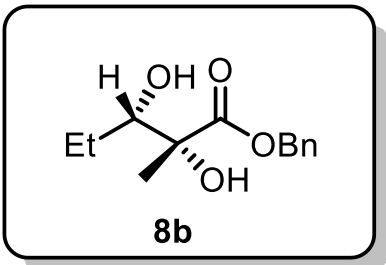

HPLC (ChiralPak AD-H, 5\% i-PrOH in hexanes, $0.8 \mathrm{~mL} / \mathrm{min}, 208 \mathrm{~nm}$ )

$\langle$ Chromatogram〉

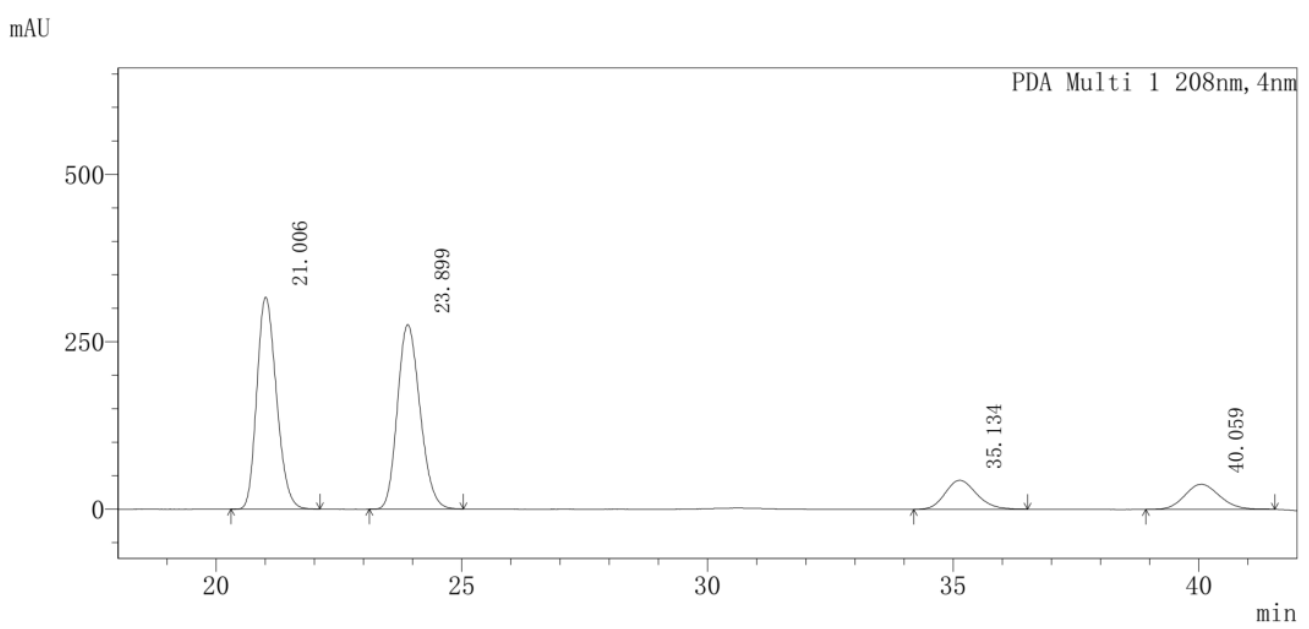

〈Peak Results〉

PDA Ch1 208nm

\begin{tabular}{|c|c|c|c|c|}
\hline Index & Time/min & Height/mAU & Quantity/Area & Area \%/\% \\
\hline 1 & 21.006 & 316585 & 8775009 & 41.058 \\
\hline 2 & 23.899 & 275534 & 8820082 & 41.269 \\
\hline 3 & 35.134 & 43328 & 1925971 & 9.012 \\
\hline 4 & 40.059 & 37646 & 1850967 & 8.661 \\
\hline
\end{tabular}

$\langle$ Chromatogram〉

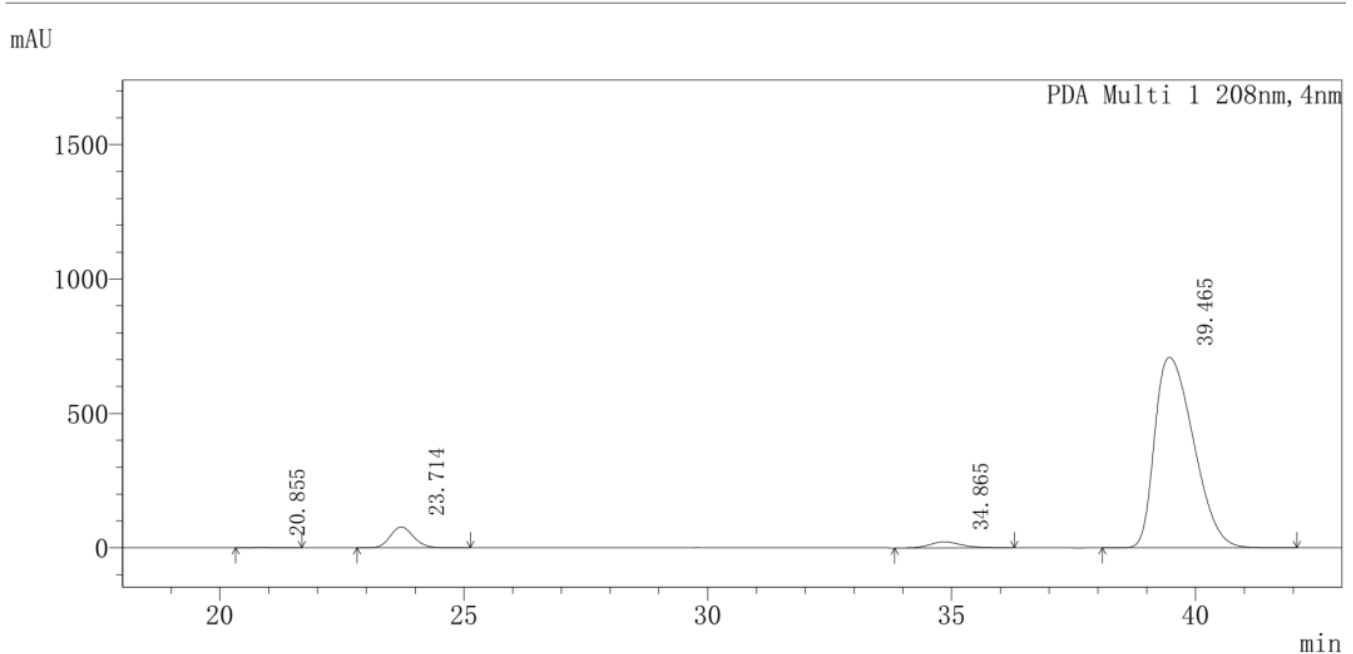

〈Peak Results〉

PDA Ch1 208nm

\begin{tabular}{|c|c|c|c|c|}
\hline Index & Time/min & Height/mAU & Quantity/Area & Area \%/\% \\
\hline 1 & 20.855 & 1304 & 39968 & 0.093 \\
\hline 2 & 23.714 & 77528 & 2545492 & 5.949 \\
\hline 3 & 34.865 & 22320 & 986479 & 2.306 \\
\hline 4 & 39.465 & 708365 & 39215546 & 91.652 \\
\hline
\end{tabular}




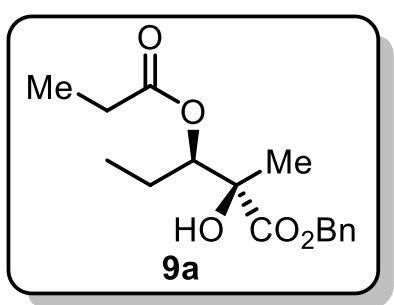

HPLC (ChiralPak OJ-H, 5\% i-PrOH in hexanes, $1.0 \mathrm{~mL} / \mathrm{min}, 209 \mathrm{~nm}$ )

$\langle$ Chromatogram〉

mAU

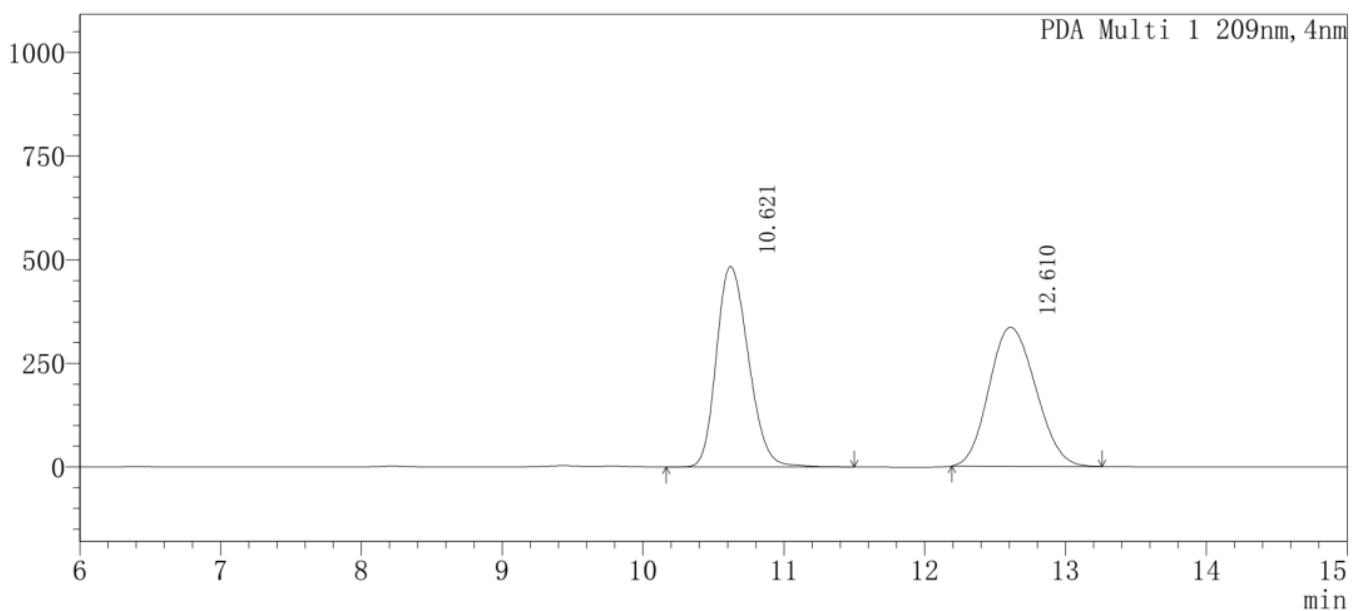

$\langle$ Peak Results >

PDA Ch1 209nm

PDA Ch1 $209 \mathrm{~nm}$
\begin{tabular}{|c|c|c|c|c|}
\hline Index & Time/min & Height/mAU & Quantity/Area & Area \%/\% \\
\hline 1 & 10.621 & 483583 & 7645047 & 49.786 \\
\hline 2 & 12.610 & 335014 & 7710711 & 50.214 \\
\hline
\end{tabular}

〈Chromatogram〉

$\mathrm{mAU}$

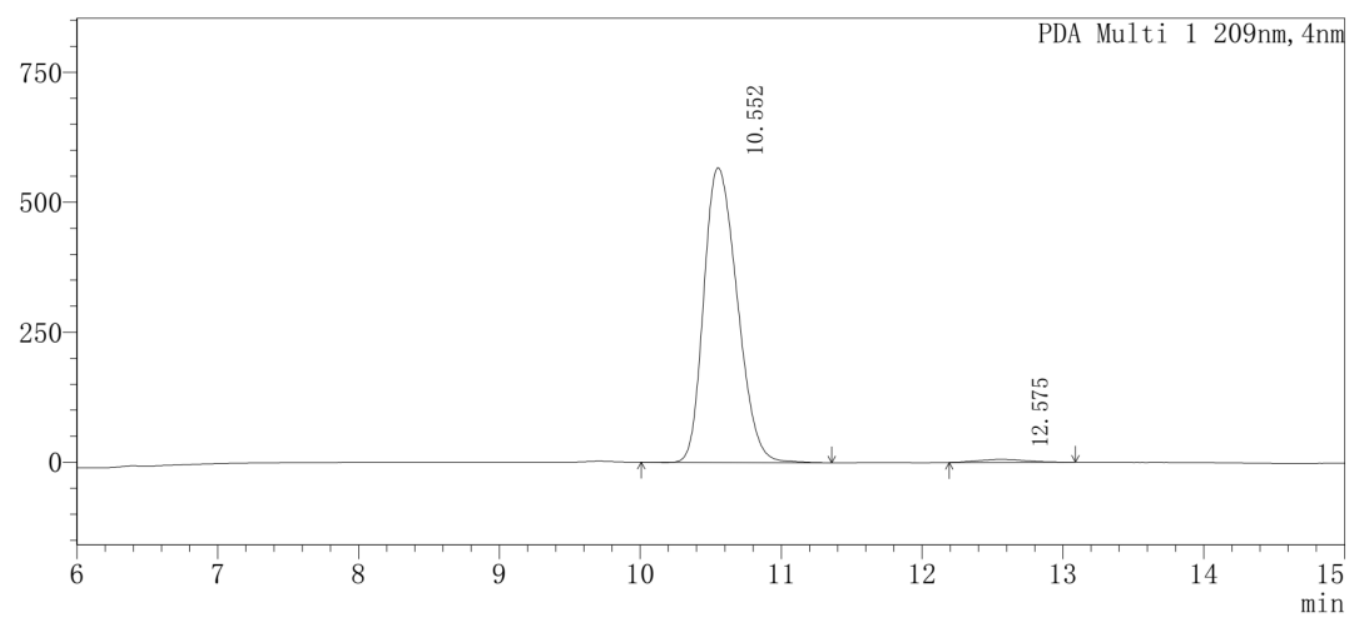

〈Peak Results〉

PDA Ch1 209nm

\begin{tabular}{|c|c|c|c|c|}
\hline Index & Time/min & Height/mAU & Quantity/Area & Area \%/\% \\
\hline 1 & 10.552 & 566824 & 9349843 & 98.608 \\
\hline 2 & 12.575 & 5465 & 131966 & 1.392 \\
\hline
\end{tabular}




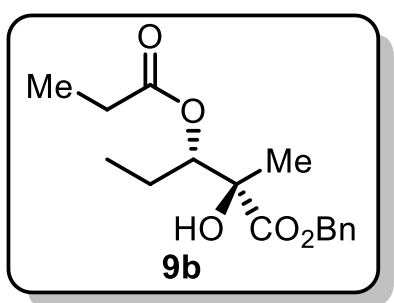

HPLC (ChiralPak OJ-H, 5\% i-PrOH in hexanes, $1.0 \mathrm{~mL} / \mathrm{min}, 209 \mathrm{~nm}$ )

$\langle$ Chromatogram〉

$\mathrm{mAL}$

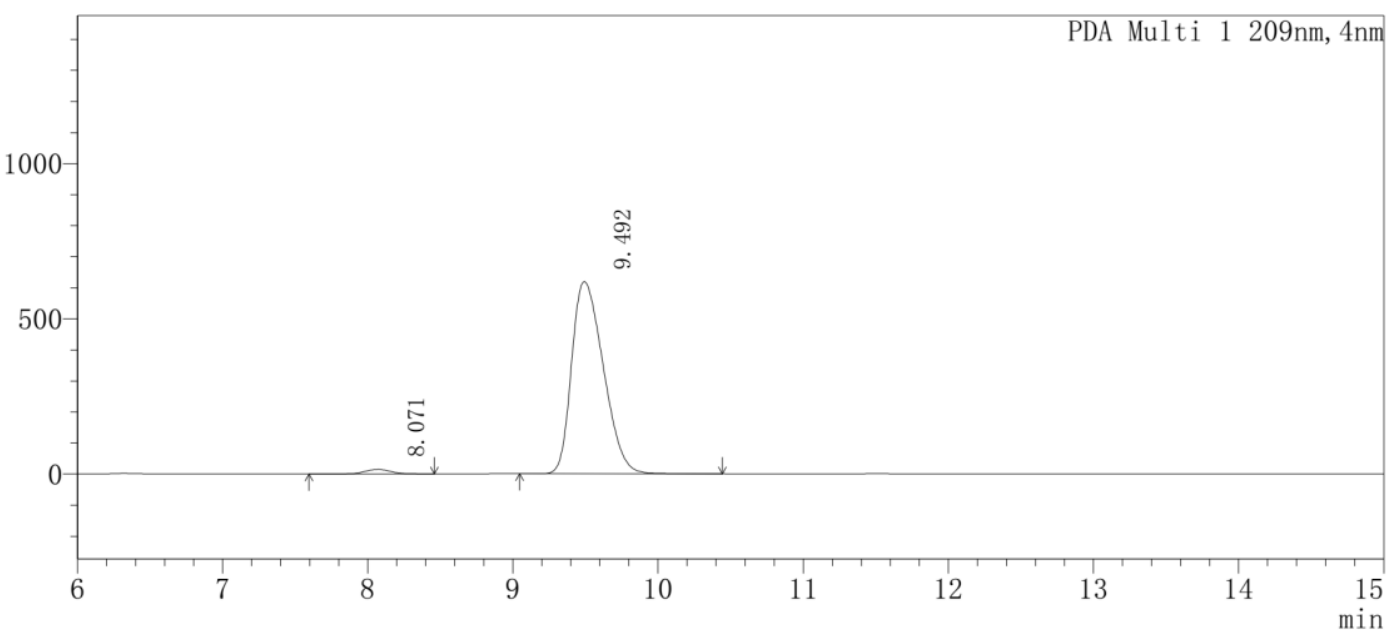

〈Peak Results〉

PDA Ch1 209nm

\begin{tabular}{|c|c|c|c|c|}
\hline Index & Time/min & Height/mAU & Quantity/Area & Area \%/\% \\
\hline 1 & 8.071 & 14213 & 177309 & 1.853 \\
\hline 2 & 9.492 & 619165 & 9390226 & 98.147 \\
\hline
\end{tabular}




\section{Supplementary References}

S1. Kalaitzakis, D.; Kambourakis, S.; Rozzell, D. J.; Smonou, I. Stereoselective chemoenzymatic synthesis of sitophilate: A natural pheromone. Tetrahedron: Asymmetry. 2007, 18, 2418.

S2. Kurouchi, H.; Sugimoto, H.; Otani, Y.; Ohwada, T. Cyclization of arylacetoacetates to indene and dihydronaphthalene derivatives in strong acids. Evidence for involvement of further protonation of $\mathrm{O}$, O-diprotonated $\beta$-ketoester, leading to enhancement of cyclization. J. Am. Chem. Soc. 2010, 132, 807.

S3. Gao, L.; Kang, B. C.; Hwang, G.-S.; Ryu, D. H. Enantioselective synthesis of $\alpha$-alkyl- $\beta$-ketoesters: Asymmetric roskamp reaction catalyzed by an oxazaborolidinium ion. Angew. Chem. Int. Ed. 2012, 51, 8322.

S4. Darses, B.; Michaelides, I. N.; Sladojevich, F.; Ward, J. W.; Rzepa, P. R.; Dixon, D. J. Expedient construction of the [7-5-5] all-carbon tricyclic core of the daphniphyllum alkaloids daphnilongeranin B and daphniyunnine D. Org. Lett. 2012, 14, 1684.

S5. Cossy, J.; Thellend, A. A convenient synthesis of $S-(\alpha-\mathrm{D}-$ and $S$-( $\beta$-D)-glucopyranosyl-6-thiomaltodextrins. Synthesis 1989, 753.

S6. Xiang, D.; Wang, K.; Liang Y.; Zhou, G.; Dong, D. A facile and efficient synthesis of polyfunctionalized pyridin-2(1H)-ones from $\beta$-oxo amides under vilsmeier conditions. Org. Lett. 2008, 10,345 .

S7. Zhang, L.; Liu, S.; Zhao, Z.; Su, H.; Hao, J.; Wang, Y. (Salen)Mn(III)-catalyzed chemoselective acylazidation of olefins. Chem. Sci. 2018, 9, 6085.

S8. Bertogg, A.; Hintermann, L.; Huber, D. P.; Perseghini, M.; Sanna, M.; Togni, A. Substrate range of the titanium TADDOLate catalyzed asymmetric fluorination of activated carbonyl 
compounds. Helvetica Chimica Acta 2012, 95, 353.

S9. Hu, S.; Zhang, L.; Li, J.; Luo, S.; Cheng, J.-P. Chiral primary amine catalyzed asymmetric direct cross-aldol reaction of acetaldehyde. Eur. J. Org. Chem. 2011, 3347.

S10. Smith, A. M. R.; Rzepa, H. S.; White, A. J. P.; Billen, D.; Hii, K. K. Delineating origins of stereocontrol in asymmetric Pd-catalyzed $\alpha$-hydroxylation of 1,3-ketoesters. J. Org. Chem. 2010, $75,3085$.

S11. Baucherel, X.; Levoirier, E.; Uziel, J.; Juge, S. Monohydroxylation of cyclic and acyclic $\beta$-keto esters with molecular oxygen catalyzed by cobalt(II) chloride in neutral conditions. Tetrahedron Lett. 2000, 41, 1385.

S12. Yu, S. -M.; Cui, K.; Lv, F.; Yang, Z. -Y.; Yao, Z. -J. Aerobic $\alpha$-hydroxylation of $\beta$-keto esters and amides by co-catalysis of $\mathrm{SmI}_{3}$ and $\mathrm{I}_{2}$ under mild base-free conditions. Tetrahedron Lett. 2016, $57,2818$.

S13. Wang, D.; Xu, C.; Zhang, L.; Luo, S. Asymmetric $\alpha$-benzoyloxylation of $\beta$-ketocarbonyls by a chiral primary amine catalyst. Org. Lett. 2015, 17, 576.

S14. Colladon, M.; Scarso, A.; Sgarbossa, P.; Michelin, R. A.; Strukul, G. Regioselectivity and diasteroselectivity in $\mathrm{Pt}(\mathrm{II})$-mediated "green" catalytic epoxidation of terminal alkenes with hydrogen peroxide: Mechanistic insight into a peculiar substrate selectivity. J. Am. Chem. Soc. 2007, 129, 7680

S15. Xu, S.; Wang, Z.; Li, Y.; Zhang, X.; Wang, H.; Ding, K. Mechanistic investigation of chiral phosphoric acid catalyzed asymmetric Baeyer-Villiger reaction of 3-substituted cyclobutanones with $\mathrm{H}_{2} \mathrm{O}_{2}$ as the Oxidant. Chem. - Eur. J. 2010, 16, 3021.

S16. (1) Frisch, M. J.; Trucks, G. W.; Schlegel, H. B.; Scuseria, G. E.; Robb, M. A.; Cheeseman, J. 
R.; Scalmani, G.; Barone, V.; Mennucci, B.; Petersson, G. A.; Nakatsuji, H.; Caricato, M.; Li, X.; Hratchian, H. P.; Izmaylov, A. F.; Bloino, J.; Zheng, G.; Sonnenberg, J. L.; Hada, M., Ehara, M.; Toyota, K.; Fukuda, R.; Hasegawa, J.; Ishida, M.; Nakajima, T.; Honda, Y.; Kitao, O.; Nakai, H.; Vreven, T.; Montgomery, J. A.; Peralta, Jr.; J. E.; Ogliaro, F.; Bearpark, M.; Heyd, J. J.; Brothers, E.; Kudin, K. N.; Staroverov, V. N.; Kobayashi, R.; Normand, J.; Raghavachari, K.; Rendell, A.; Burant, J. C.; Iyengar, S. S.; Tomasi, J.; Cossi, M.; Rega, N.; Millam, J. M.; Klene, M.; Knox, J. E.; Cross, J. B.; Bakken, V.; Adamo, C.; Jaramillo, J.; Gomperts, R.; Stratmann, R. E.; Yazyev, O.; Austin, A. J.; Cammi, R.; Pomelli, C.; Ochterski, J. W.; Martin, R. L.; Morokuma, K.; Zakrzewski, V. G.; Voth, G. A.; Salvador, P.; Dannenberg, J. J.; Dapprich, S.; Daniels, A. D.; Farkas, O.; Foresman, J. B.; Ortiz, J. V.; Cioslowski, J.; Fox, D. J. Gaussian 09, Revision E.01, Gaussian, Inc., Wallingford CT, 2009.

S17. Vosko, S. H.; Wilk, L.; Nusair, M. Accurate spin-dependent electron liquid correlation energies for local spin density calculations: a critical analysis. Can. J. Phys. 1980, 58, 1200.

S18. Lee, C.; Yang, W.; Parr, R. G. Development of the Colle-Salvetti correlation-energy formula into a functional of the electron density. Phys. Rev. B 1988, 37, 785.

S19. Becke, A. D. Density-functional thermochemistry. III. The role of exact exchange. J. Chem. Phys. 1993, 98, 5648.

S20. Stephens, P. J.; Devlin, F. J.; Chabalowski, C. F.; Frisch, M. J. Ab initio calculation of vibrational absorption and circular dichroism spectra using density functional force fields. J. Phys. Chem. 1994, 98, 11623.

S21. Tomasi, J.; Mennucci, B.; Cammi, R. Quantum mechanical continuum solvation models. Chem. Rev. 2005, 105, 2999. 
S22. Grimme, S.; Antony, J.; Ehrlich, S.; Krieg, H. A consistent and accurate ab initio parametrization of density functional dispersion correction (DFT-D) for the 94 elements H-Pu. $J$. Chem. Phys. 2010, 132, 154104.

S23. Grimme, S.; Ehrlich, S.; Goerigk, L. Effect of the damping function in dispersion corrected density functional theory. J. Comput. Chem. 2011, 32, 1456.

S24. Johnson, E. R.; Becke, A. D. A post-Hartree-Fock model of intermolecular interactions: Inclusion of higher-order corrections. J. Chem. Phys. 2006, 124, 174104.

S25. Johnson, E. R.; Becke, A. D. A post-Hartree-Fock model of intermolecular interactions. $J$. Chem. Phys. 2005, 123, 024101.

S26. Weigend, F.; Ahlrichs, R. Balanced basis sets of split valence, triple zeta valence and quadruple zeta valence quality for $\mathrm{H}$ to Rn: Design and assessment of accuracy. Phys. Chem. Chem. Phys. 2005, 7, 3297.

S27. CYLview, 1.0b; Legault, C. Y. Université de Sherbrooke, 2009 (http://www.cylview.org) 